Publishing Reproducible Geoscientific Papers: Status quo, benefits, and opportunities

Markus Konkol 

UNIVERSITY OF MÜNSTER

Geoinformatics

\section{Publishing Reproducible Geoscientific Papers: Status quo, benefits, and opportunities}

Inaugural dissertation in fulfillment of the academic degree of

Doctor of Natural Sciences (Dr. rer. nat.)

in the Department of Geosciences

of the Faculty of Mathematics and Natural Sciences

of the Westfälische Wilhelms-Universität Münster

Submitted by

Markus Konkol

from Münster, Germany

April 1, 2019 
Dean: Prof. Dr. Harald Strauß

First supervisor: Prof. Dr. Christian Kray

Second supervisor: Prof. Dr. Carsten Keßler

Day of defence:

Day of promotion: 


\section{Acknowledgement}

I would like to take the opportunity to thank a couple of people for their support that I received during this work. First of all, special thanks to my supervisor Prof. Dr. Christian Kray who always helped me with constructive discussions, advice, and numerous helpful suggestions. Also, special thanks to my second supervisor, Prof. Dr. Carsten Keßler, for the feedback before submitting this thesis. I would like to thank Prof. Dr. Edzer Pebesma and Dr. Max Pfeiffer for a couple of inspiring discussions.

I am grateful for the productive and successful work with my two o2r colleagues Daniel and Marc. I am looking forward to working with you on o2r2. I am also thankful for all the implementations done by Jan Suleiman during his work as a student assistant. This work made my life much more comfortable. Thanks to Karsten and Heike for their support with all the organizational work. Thank you, former and current members of the Sitcom lab and IfGI, for all the feedback I received from you concerning papers and presentations. Particularly, I am thankful to Ana and Samual for designing the beautiful cover. I would also like to thank Dr. Celeste R. Brennecka for proofreading some of my papers and for giving so many helpful comments.

Finally, the people outside IfGI: Thanks to my family, particularly my parents Elisabeth and Richard, for their support (Vielen Dank an meine Familie, vor allem Elisabeth und Richard, für eure Unterstützung). Thanks to my friends and the Ph.D. students I met during the last years for all the interesting conversations.

This dissertation was partially funded by the Institute for Geoinformatics at the University of Münster and Deutsche Forschungsgemeinschaft, Grant number: KR 3930/3-1.

\section{Da $\begin{aligned} & \text { Deutsche } \\ & \text { Forschungsgemeinschaft }\end{aligned}$}

ifgi

Institute for Geoinformatics

University of Münster 



\section{Abstract}

Open reproducible research (ORR) is the practice of publishing the source code and the datasets that are needed to produce the computational results reported in a paper. Since geoscientific articles often include geostatistical analyses and spatiotemporal data, reproducibility should be a cornerstone of the computational geosciences but is rarely realized. In addition, the current way of publishing scientific outcomes, i.e. as static PDFs, does not adequately report on computational aspects. Thus, readers cannot fully understand how the authors came to the conclusions and how robust these are to changes in the analysis. Consequently, it is difficult for reviewers to follow the analysis steps, and for other researchers to reuse existing materials. This dissertation has two overarching goals to tackle these issues: First, it aims at assisting authors in adhering to ORR principles to ensure high scientific standards. The second goal is to reap the benefits that come with papers supplemented by code and data. To achieve these objectives, this thesis reports on five complementary studies to collect and analyze qualitative (i.e. through interviews and a focus group) and quantitative data (i.e. through surveys and reproducibility studies). Based on these studies, this work provides four key contributions: First, it identifies obstacles that prevented geoscientists from publishing ORR. To overcome these barriers, this dissertation suggests concrete and directly applicable strategies. One of these strategies is the executable research compendium (ERC) which encapsulates the paper, code, data, and the entire software environment needed to produce the computational results. Based on that, this work presents the design and implementation of an ERC-based workflow. It allows authors to convey their computational methods and results by also providing interactive access to code and data, and readers to deeply investigate the computational analysis while reading the actual article, e.g. by changing the parameters of the analysis. Finally, this work describes the concept of a binding; a binding connects those code lines and data subsets that produce a specific result, e.g. a figure or number. By also considering user interface widgets (e.g. a slider), this approach allows readers to interactively manipulate the parameters of the analysis to see how these changes affect the result. To conclude, the contributions include (i) a set of obstacles which prevent geoscientists from publishing ORR, (ii) concepts and tools to overcome the identified barriers, (iii) incentives and opportunities that come with attached code and data, and (iv) solutions to realize the incentives which eventually result in a higher number of open and reproducible research. 



\section{List of Abbreviations}

\begin{tabular}{|c|c|}
\hline API & Application Program Interface \\
\hline AGILE & $\begin{array}{l}\text { Association of Geographic Information Laboratories for } \\
\text { Europe }\end{array}$ \\
\hline CRAN & Comprehensive R Archive Network \\
\hline DOI & Digital Object Identifier \\
\hline EGU & European Geosciences Union \\
\hline$(\mathrm{U})(\mathrm{R})(\mathrm{P}) \mathrm{ERC}$ & $\begin{array}{l}\text { (Unvalidated) (Reviewed) (Published) Executable Re- } \\
\text { search Compendium }\end{array}$ \\
\hline GIScience & Geographic information science \\
\hline GPS & Global Positioning System \\
\hline HCI & Human-computer interaction \\
\hline HTML & Hypertext Markup Language \\
\hline JSON & JavaScript Object Notation \\
\hline $02 \mathbf{r}$ & Opening Reproducible Research (project name) \\
\hline OJS & Open Journal Systems \\
\hline ORCID & Open Researcher and Contributor ID \\
\hline ORR & Open reproducible research \\
\hline OSF & Open Science Framework \\
\hline PDF & Portable Document Format \\
\hline REP & Reproducibility Enhancement Principles \\
\hline TOP & Transparency and Openness Promotion guidelines \\
\hline UI & User interface \\
\hline VGI & Volunteered geographic information \\
\hline XML & Extensible Markup Language \\
\hline
\end{tabular}





\section{List of Publications}

This thesis is a cumulative dissertation composed of articles published and under review in several outlets (see Table 0.1). Chapter 2 corresponds to P2, chapter 3 to P3, chapter 4 to P1, chapter 5 to P4, and chapter 6 to P5.

\begin{tabular}{|c|c|c|c|}
\hline No. & Publication Details & Outlet & Status \\
\hline P1 & $\begin{array}{l}\text { Konkol, Markus, Daniel Nüst, Edzer Pebesma, } \\
\text { Christian Kray, Marc Schutzeichel, Hol- } \\
\text { ger Przibytzin, and Jörg Lorenz (2017). } \\
\text { Opening the publication process with exe- } \\
\text { cutable research compendia. D-Lib Maga- } \\
\text { zine, 23(1/2). http://www.dlib.org/dlib/ } \\
\text { january17/nuest/01nuest.html }\end{array}$ & Journal & Published \\
\hline P2 & $\begin{array}{l}\text { Nüst, Daniel, Carlos Granell, Barbara Hofer, } \\
\text { Markus Konkol, Frank O. Ostermann, Rusne } \\
\text { Sileryte, and Valentina Cerutti (2018). Repro- } \\
\text { ducible research and GIScience: an evaluation } \\
\text { using AGILE conference papers. PeerJ, 6:e5072. } \\
\text { https://doi.org/10.7717/peerj.5072 }\end{array}$ & Journal & Published \\
\hline P3 & $\begin{array}{l}\text { Konkol, Markus, Christian Kray, and Max Pfeif- } \\
\text { fer (2018). Computational reproducibility in } \\
\text { geoscientific papers: Insights from a series of } \\
\text { studies with geoscientists and a reproduction } \\
\text { study. International Journal of Geographical In- } \\
\text { formation Science, 1-22. https://doi.org/10. } \\
\text { 1080/13658816.2018.1508687 }\end{array}$ & Journal & Published \\
\hline P4 & $\begin{array}{l}\text { Konkol, Markus and Christian Kray (2018). In- } \\
\text { depth examination of spatiotemporal figures in } \\
\text { open reproducible research. Cartography and } \\
\text { Geographic Information Science, 1-16. https: // } \\
\text { doi.org/10.1080/15230406.2018.1512421 }\end{array}$ & Journal & Published \\
\hline P5 & $\begin{array}{l}\text { Konkol, Markus, Christian Kray, and Jan } \\
\text { Suleiman (2018). Creating interactive scien- } \\
\text { tific publications using bindings. Proceedings } \\
\text { of the ACM on Human-Computer Interaction. } \\
\text { https://doi.org/10.31223/osf.io/jtd8c }\end{array}$ & Journal & $\begin{array}{l}\text { Under re- } \\
\text { view }\end{array}$ \\
\hline
\end{tabular}

Tab. 0.1: List of publications that are part of this dissertation. 



\section{Contents}

1 Introduction 1

1.1 Motivation . . . . . . . . . . . . . . . . 1

1.2 Scope and research questions $\ldots \ldots \ldots \ldots$

1.2 .1 Scope ...................... 4

1.2 .2 Research questions . . . . . . . . . . . . . . . 4

1.3 Methodology ..................... 6

1.4 Key contributions . . . . . . . . . . . . . . . . 8

1.5 Thesis outline . . . . . . . . . . . . . . . . 12

2 Reproducible research and GIScience: an evaluation using AGILE conference papers 13

2.1 Introduction . . . . . . . . . . . . . . . . . . . 13

2.2 Related Work . . . . . . . . . . . . . . . . . . . . . 15

2.3 Materials and Methods . . . . . . . . . . . . . . . . 16

2.3.1 The paper corpus . . . . . . . . . . . 16

2.3 .2 Assessment of reproducibility . . . . . . . . . . . 17

2.4 Results . . . . . . . . . . . . . . . . . . . 23

2.4 .1 Assessment of reproducibility . . . . . . . . . . . 23

2.4 .2 Author feedback (survey) . . . . . . . . . . . . . 27

2.5 Discussion . . . . . . . . . . . . . . . . . . . . . 29

2.5.1 A critical review of this paper's reproducibility . . . . . . . 29

2.5.2 Improving day-to-day research in GIScience . . . . . . . . . 31

2.5 .3 Suggestions to authors . . . . . . . . . . . . . . 31

2.5.4 Recommendations to conferences in GIScience and organizations like AGILE . . . . . . . . . . . . . . . . . . 32

2.6 Conclusions . . . . . . . . . . . . . . . . . . 34

2.7 Acknowledgements . . . . . . . . . . . . . . . 35

3 Computational reproducibility in geoscientific papers: Insights from a $\begin{array}{ll}\text { series of studies with geoscientists and a reproduction study } & 37\end{array}$

3.1 Introduction . . . . . . . . . . . . . . . . 38

3.2 Related Work . . . . . . . . . . . . . . . . . . . . 39

3.2.1 Reproducible research . . . . . . . . . . . . . . . . 39

3.2.2 Incentives for publishing reproducible research $\ldots \ldots . . .440$ 
3.2.3 Reasons for unreproducible papers . . . . . . . . . . . . . 41

3.2.4 Guidelines and recommendations . . . . . . . . . . . . . 41

3.2.5 Reproducible research in the geosciences . . . . . . . . . . . 42

3.3 Methods . . . . . . . . . . . . . . . . . . . 43

3.3.1 Approaches . . . . . . . . . . . . . . . 44

3.3 .2 Online survey . . . . . . . . . . . . . . . . . 44

3.3.3 Semi-structured interviews . . . . . . . . . . . 45

3.3 .4 Focus Group . . . . . . . . . . . . . . . . . . . . . 48

3.3.5 Reproducing geoscientific results . . . . . . . . . . . . 49

3.4 Discussion . . . . . . . . . . . . . . . . . 55

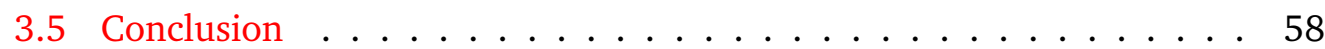

4 Opening the publication process with executable research compendia 61

4.1 Introduction . . . . . . . . . . . . . . . . 61

4.2 Related Work . . . . . . . . . . . . . . . . . . . 62

4.2.1 Definition of Reproducibility . . . . . . . . . . . . . . 63

4.2.2 Open Data and Open Access . . . . . . . . . . . . . . . 63

4.2.3 Research Compendia and Research Objects . . . . . . . . . 64

4.2.4 Research Data Platforms . . . . . . . . . . . . . . . . . 65

4.2.5 Capture Workflows and Runtime Environments . . . . . . . . 65

4.2.6 Challenges in Open Reproducible Research . . . . . . . . . . . 67

4.3 The Executable Research Compendium . . . . . . . . . . . . . . . . 68

4.3.1 Stakeholders and Roles in the Publication Process . . . . . . . 68

4.3.2 Core Parts of ERC . . . . . . . . . . . . . . . . . . . . 69

4.3 .3 Creation and Reproducibility . . . . . . . . . . . 70

4.3.4 Publication Process . . . . . . . . . . . . . . . . 72

4.3 .5 Findability and Preservation . . . . . . . . . . . 73

4.3.6 Interaction, Exploration, and Reuse . . . . . . . . . . . 75

4.4 Discussion . . . . . . . . . . . . . . . . . 76

4.4.1 Approach ................... 76

4.4 .2 Distinction of ERC . . . . . . . . . . . 76

4.4 .3 Creation and Usage . . . . . . . . . . . . . . . 77

4.4 .4 Challenges . . . . . . . . . . . . . 78

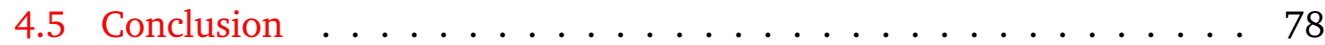

4.6 Future Work . . . . . . . . . . . . . . . . . . . . 79

5 In-depth examination of spatiotemporal figures in open reproducible $\begin{array}{ll}\text { research } & \mathbf{8 1}\end{array}$

5.1 Introduction . . . . . . . . . . . . . . . 81

5.2 Related Work . . . . . . . . . . . . . . . . . . 83

5.2.1 Working with scientific publications . . . . . . . . . 83

5.2 .2 Reproducible research . . . . . . . . . . . . . . 83 
5.2 .3 Figures in scientific publications . . . . . . . . . . . . 84

5.2 .4 Interactive figures $\ldots \ldots \ldots \ldots . \ldots . \ldots . \ldots$

5.3 Methodology . . . . . . . . . . . . . . . . . 85

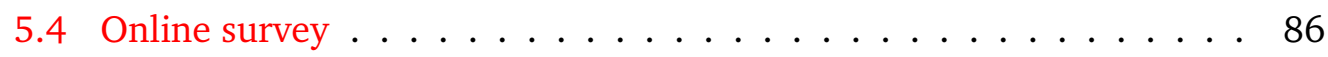

5.4 .1 Materials ................... 86

5.4 .2 Procedure . . . . . . . . . . . . . 87

5.4 .3 Results . . . . . . . . . . . . . . . . . 87

5.5 Semi-structured interviews . . . . . . . . . . . . . . . . . 89

5.5 .1 Materials . . . . . . . . . . . . . . . 89

5.5 .2 Procedure .......................... 89

5.5 .3 Results . . . . . . . . . . . . . . . . . . 91

5.6 Focus group . . . . . . . . . . . . . . . . . . 94

5.6 .1 Procedure . . . . . . . . . . . . . . . . 94

5.6 .2 Results . . . . . . . . . . . . . . . . . . . . 94

5.7 The extended workflow for reproducible research papers . . . . . . . 95

5.8 Realization of the workflow . . . . . . . . . . . . . . . 97

5.9 Discussion . . . . . . . . . . . . . . . . . . . . . . . 99

5.10 Conclusion . . . . . . . . . . . . . . . . 102

6 Creating interactive scientific publications using bindings 105

6.1 Introduction . . . . . . . . . . . . . . . . . . . 105

6.2 Related Work . . . . . . . . . . . . . . . . . . 107

6.2.1 Publishing research materials . . . . . . . . . . . 107

6.2 .2 Connecting paper, code, and data . . . . . . . . . . . 108

6.2 .3 Creating interactive papers . . . . . . . . . 110

6.3 Binding . . . . . . . . . . . . . . . . 111

6.3 .1 Design rationale . . . . . . . . . . . . 111

6.3 .2 Computational result . . . . . . . . . . . . . . 112

6.3 .3 Source code . . . . . . . . . . . . . . . . . . 112

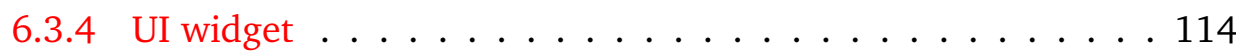

6.3 .5 Data . . . . . . . . . . . . . . . . 114

6.4 Realization . . . . . . . . . . . . . . . . . . . . 115

6.4.1 Author's perspective: Creating a binding . . . . . . . . . . 115

6.4.2 Reader's perspective: Using the interactive figure . . . . . . . 116

6.4 .3 Technical details . . . . . . . . . . . . . . . 117

6.5 Evaluation . . . . . . . . . . . . . . . . . 117

6.5 .1 Materials . . . . . . . . . . . . . . . 118

6.5 .2 Procedure . . . . . . . . . . . . . . 118

6.5 .3 Results . . . . . . . . . . . . . . . . . . . 119

6.6 Discussion . . . . . . . . . . . . . . . . . 122

6.6 .1 Parameters . . . . . . . . . . . . . . 122

6.6 .2 Effort and user interface . . . . . . . . . . . . . 123 
6.6 .3 Data . . . . . . . . . . . . . . . . . 123

6.6 .4 Benefits . . . . . . . . . . . . . . . . 124

6.6 .5 Limitations . . . . . . . . . . . . . . . . . 124

6.7 Conclusion . . . . . . . . . . . . . . . . . . 125

6.8 Future Work . . . . . . . . . . . . . . . . . . 126

7 Discussion 127

7.1 The status quo of ORR in the computational geosciences . . . . . . 127

7.2 Assisting geoscientists in publishing ORR . . . . . . . . . . . . . 129

7.3 Incentives based on open reproducible research . . . . . . . . . . 131

7.4 Creating interactive geoscientific papers using bindings . . . . . . . 133

7.5 Generalizability and limitations of the results . . . . . . . . . 135

8 Conclusion 139

9 Future Work $\quad 141$

9.1 Practical work . . . . . . . . . . . . . . . . . . . . . . 141

9.2 Research . . . . . . . . . . . . . . . . . . . . . . . . 142

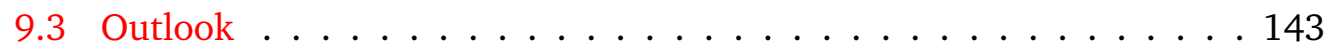

$\begin{array}{ll}\text { Bibliography } & 145\end{array}$

$\begin{array}{ll}10 \text { Supplemental material } & 161\end{array}$ 


\section{List of Figures}

1.1 Overview of the methods employed to answer the research questions RQ1 to RQ4, the resulting contributions $\mathbf{C} 1$ to $\mathbf{C} 4$, and the corresponding papers and chapters. . . . . . . . . . . . . . .

2.1 Two illustrations of the test corpus papers: word cloud, scaled and colored by the number of occurrence of words with at least 100 occurrences (96 unique words) (A); top words sorted by overall occurrence and number of papers including the word at least once (B). . . . . .

2.2 The final reproducible research criteria used for the evaluation. The categories Data, Methods (sub-categories: preprocessing, method/analysis/processing, and computational environment), and Results each have four levels ranging from $0=$ not reproducible to $3=$ fully reproducible. .

2.3 Results of reproducibility assessment across all categories for the assessment of reproducibility: Data (A), Methods with sub-categories preprocessing (B), method/analysis/processing (C), computational environment (D), and Results (E). The level of reproducibility ranges from 0 (not reproducible) to 3 (fully reproducible); For 5 conceptual papers, all categories are NA. . . . . . . . . . . . . . . .

2.4 Mean reproducibility levels per category over time; black dotted line connects the mean per year over all categories (in 2010 only one of three papers could be assessed, reaching level 1 for methods) . . . . . . 27

2.5 Author survey results on the importance of reproducibility. . . . . . . . 28

3.1 The diverging stacked bar chart (Heiberger and Robbins, 2014) shows the percentage of respondents publishing reproducible research and reproducing the work of other researchers. Numbers in brackets show absolute numbers of respondents. Percentages are grouped into the categories never/rarely, sometimes, and often/always. Note: All except the last question were only shown to authors and not to readers. . . .

3.2 Differences between the original (a) and reproduced figure (b) (Marlon et al., 2016). Design: placement of legend (blue box), aspect ratio, color of results (purple), data type of legend numbers (red). Content: labelling (grey), background map (orange), number of classes (yellow), deviating results (green). . . . . . . . . . . . . 53 
4.1 The core parts of an ERC are data, software, documentation, and UI bindings. . . . . . . . . . . . . . . . 70

4.2 The ERC-based publication process: a research workspace is prepared for a URC, which is validated to become an ERC, which turns into an RERC after peer review, and eventually published as a PERC to be used, e.g. for subsequent cycles. . . . . . . . . . . . . . .

5.1 Responses to "Relating to your last five publications, how often did you incorporate the following visualizations?" Numbers in brackets show how many responded to the item. Percentages are grouped into never/rarely, sometimes, and often/always. . . . . . . . . . .

5.2 Responses to the question: "While reading a scientific publication related to my own research, I would like to ...". Numbers in brackets show how many responded to the particular item. Percentages are grouped into strongly disagree/disagree, neither agree nor disagree, agree/strongly agree. . . . . . . . . . . . . . .

5.3 Pictures used to inspire interviewees who struggled with imagining interactive components in static papers. Left: Map of earthquake events in the Pacific (Source: https://cran.r-project.org/web/packages/ leafletR/leafletR.pdf, last access: 01.04.2019) Right: Daytime pattern of air temperature (El-Madany et al., 2016). . . . . . . . . . 90

5.4 Mockups used to inspire participants and to present the idea of a reproducible research platform. Left: research components, possibility to re-run the analysis in the lower left corner, and the static paper. Right: interactive view enriched by UI widgets to change values. . . . .

5.5 The extended workflow for examining reproducible scientific papers. The steps Discovery, Inspection (passive tasks, e.g. looking at components), Manipulation, and Substitution (active tasks, e.g. modifying parameters or replacing underlying components) each contribute to the reader's understanding. The knowledge gain is probably not equal as suggested by the step height. Comparison is considered as a crosscutting task relevant in each step. . . . . . . . . . . . . .

5.6 Implementation of the workflow step Discovery. Readers can create queries using spatial (map), temporal, thematic (keywords), and source code-specific properties (libraries). . . . . . . . . . . .

5.7 Inspection: Readers can read the paper (left) and at the same time examine the underlying source code and dataset to understand how the reported results were computed (right). . . . . . . . . . . .

5.8 Manipulation: Readers can change the model of the variogram underlying the kriging interpolation by using option buttons to see how the results change (right), and examine only those parts of the source code and data subsests that were used to produce the figure (left). . . . . . 
5.9 Substitution: Readers can substitute the dataset underlying a paper (base Executable Research Compendium, ERC) by data from another paper containing compatible data (overlay ERC). . . . . . . . . 100

6.1 Example binding stored as a JSON object containing the five components computational result, source code, UI widget, and data. The interactive figure that is generated from this binding is shown in Figure $6.3 \ldots \ldots \ldots \ldots \ldots \ldots$

6.2 Creating a binding in five steps. Top: Selecting the computational result and the source code lines which produce it (Step 1 and 2). Bottom: Specifying the parameter that should be manipulated and configuring the UI widget (Step 3 and 4). In the fifth step (not shown), authors specify the data subset (see video). . . . . . . . . . . . . . 116

6.3 In parallel to reading the actual article on the left, readers can also change the original parameter (blue box: "velocity=2.0") by using the slider on the right and see how the new value (black box: "0.4") affects the output (orange box: "damage costs"). . . . . . . . . . . . . . 117 



\section{List of Tables}

0.1 List of publications that are part of this dissertation. . . . . . . . . vii

2.1 Reproducibility-related keywords in the corpus, ordered by sum of matches per paper. For full references of the corpus papers, see supplemental material under https://doi.org/10.7717/peerj.5072/supp-1 (last access: 01.04.2019). . . . . . . . . . . . . . . . 18

2.2 Survey questions (except for paper identification questions; for full questionnaire see supplemental material under https://doi .org/10. 7717 /peerj.5072/supp-2, last access: 01.04.2019). . . . . . . . . . 24

2.3 Reproducibility levels for paper corpus; "-" means category is not available. For full references of the corpus papers see https://doi.org/10. $7717 /$ peerj.5072/supp-1 (last access: 01.04.2019). . . . . . . . . 25

2.4 Statistics of reproducibility levels per criterion. . . . . . . . . . . 27

2.5 Mean levels per criterion for full and short papers. . . . . . . . . . 28

2.6 Hindering circumstances for reproducibility for each survey response (n =17) sorted by barrier type for the category with most "Main reason" occurrences; each line is one response. . . . . . . . . . . .

3.1 Obstacles while publishing reproducible research (left) and while reproducing other researchers' work (right). Numbers in brackets show how many interviewees mentioned the obstacle. Aspects marked with (*) were also mentioned in the focus group discussion. . . . . . . . . .

3.2 Issues we encountered during code execution. Numbers in brackets show how often and in how many papers they occurred (overall occurrence/number of papers). In total, we encountered technical issues in 39 papers. . . . . . . . . . . . . . . . . . .

3.3 Number of design and content differences between original and reproduced figures. Bolded numbers in brackets show how many figures had the corresponding figure component. Italic numbers in brackets show in how many papers the differences occurred. . . . . . . . . . . .

5.1 Requested tasks while examining a paper assigned to the four workflow steps. Numbers in brackets indicate how many mentioned the task (\#interviewees; \#focusGroupParticipants). . . . . . . . . . . . . . 92 



\section{Introduction}

\subsection{Motivation}

The primary goal of a scientist is to create and disseminate new domain knowledge. A research article describes these new insights and how they were achieved, for example, by providing all details about the experimental setup employed. One of the essential tools for a scientist's everyday work is the computer. They allow researchers to process a large amount of data and to run complex analyses within a short time. These opportunities eventually result in more research output and accelerate scientific progress compared to the time before computers emerged. We can thus note that the advent of computers has changed research drastically. Nowadays, a large number of scientific papers report on in silico experiments, i.e. computational research based on software and data. Despite these technical developments, the way how research is presented to others has not changed accordingly. John Claerbout, a geophysicist, reconsidered whether the traditional way of publishing research, i.e. as static PDF files and websites, is still suitable for the field of computational science. Buckheit and Donoho (1995, p. 5) summarized his principles as follows:

"An article about computational science in a scientific publication is not the scholarship itself, it is merely advertising of the scholarship. The actual scholarship is the complete software development environment and the complete set of instructions which generated the figures."

This statement has implications for computational research in general and notably the geosciences. Many geoscientific articles include geostatistical analyses or models based on algorithms that run the computations on data (Goodchild, 1992). In these cases, a manuscript including a convincing rationale as well as a textual description of the computational steps and the data is not sufficient (Gil et al., 2016). Instead, readers should be able to access the "actual scholarship", i.e. the source code and the data that were used to generate the results in the paper. Access to the materials is especially crucial for reviewers who aim at verifying the new insights before publication. In addition to the inspection of the materials, this verification process should also include the possibility to re-run the computational steps specified in the 
source code scripts (Stodden et al., 2016). One approach to realizing these tasks is the concept of a research compendium (Gentleman and Lang, 2007). Besides the article, it contains the code and data files used to produce the reported results. In this dissertation, the practice of publishing re-computable research results is called "open reproducible research" (ORR). In particular, ORR means that all materials, i.e. source code, data, and software are publicly available enabling others to produce the same results as reported in the scientific publication, e.g. figures, tables, and statistical numbers (Goodman et al., 2016). This form of publishing computational research is part of a broader concept called "open science" (Nosek et al., 2015) which comprises several initiatives, such as "open data" (Gewin, 2016) and "open code" (Easterbrook, 2014). "Open science" and ORR are not only beneficial for the peer review process but also for readers and authors. Readers might not want to develop a computational analysis from scratch but rather reuse and build upon existing materials resulting in citations for the original author and less work for the reader (Vandewalle, 2012).

Despite these benefits, several reproducibility studies across different research fields concluded that open reproducible research is still not common practice (cf. Collberg and Proebsting, 2016; Iqbal et al., 2016; Ostermann and Granell, 2017). Reasons for that are manifold and often refer to the used materials: For example, the authors do not see the benefits that warrant the extra effort required to prepare the materials for publication (Barnes, 2010). As a result, disciplines such as psychology, medicine, or biology suffer from a so-called "reproducibility crisis" (Baker, 2016a). This crisis might also affect the credibility of the geosciences making it easy for climate change deniers to put research results into question. For this reason, journals and funding agencies increasingly demand access or links to the source code scripts and datasets used (Reichman et al., 2011; Skaggs et al., 2015).

Nevertheless, the main output of a research endeavor is still the scientific article alone although describing the computational steps and the data is hardly achievable with pure text (Gentleman and Lang, 2007). Besides this drawback, the way a research paper is presented to readers, i.e. as a PDF or HTML rendering, has not yet adapted to facilitate inspection of the used materials, such as source code and data (Pham et al., 2012). These materials are wrapped into a folder and attached to the article or stored in external repositories, if available at all. Readers have to download the folder or visit the repository and can then examine the included materials. Aside from these efforts, another drawback of this approach is that the results in the paper and the materials are disconnected (Pham et al., 2012). Reviewers and readers need to handle three separate components: the actual paper and several potentially complex source code scripts and data files. While reading an article, it remains unclear which parts of the code and which data were used to compute a specific figure, table, or statistic. Readers and reviewers who are curious about how a specific 
result was computed have to search for the right source code lines and data subsets. Understanding the materials used to produce the results can thus still be a complex and daunting task. As a consequence, readers might not want to invest time in understanding how the code works. Such difficulties also prevent researchers from reusing existing materials. This issue, in turn, lowers the incentives for authors to publish code and data eventually resulting in less reproducible research.

A solution to these issues might be to change the way how readers consume published research. Increasingly, papers published as HTML renderings allow some fundamental interactions, such as clicking on references and downloading figures or tables. Except for a few experimental attempts where readers could interact with so-called "living figures" (Singh Chawla, 2015), the source code and the data have not been considered so far. However, these materials can be used to create interactive publications which provide more advanced interactions resulting in better access to code and data. Parallel to reading a scientific article, readers and reviewers could inspect the code and the data that were used to produce a specific result. Referring back to Claerbout's principles, figures play an important role during this inspection task as they frequently visualize key results and are frequently produced by code and data (Claerbout and Karrenbach, 1992). Again, this is also true for geoscientific papers of which many include spatiotemporal analyses and geostatistics illustrated in maps or time series (Giraud and Lambert, 2017). Besides clicking on references and downloading figures, readers and reviewers might also be interested in modifying the analysis underlying the results in the paper to see if the results still hold for different parameter values. This task could be achieved, for example, by manipulating the initial values of the parameters in the source code with the help of user interface (UI) widgets, such as a slider. Afterward, readers can observe changes in the re-computed figure. Nevertheless, creating such interactive (geo)scientific publications in order to make research results more transparent requires extensive effort, either from authors or journal staff. Besides, the low number of fully reproducible publications, an essential requirement to realize the features mentioned above, is still an obstacle.

To conclude, there are three critical issues in geoscientific research: First, open reproducible research is a fundamental problem and rarely practiced. Notably, we know little about computational reproducibility in the geosciences, i.e. what exactly prevents geoscientists from publishing ORR. To fill this gap, this thesis starts with collecting insights into the current state of ORR in the computational geosciences including, for example, geographic information science (GIScience), landscape ecology, and further disciplines that use spatiotemporal analyses and data (see, for example, Nature Geosciences (2018) for a list of geoscientific disciplines). Based on these findings, this dissertation provides a set of concrete and directly applicable recommendations, concepts, and tools to increase the number of reproducible papers 
in the geosciences. Second, researchers do not see the incentives to invest time in the publication of ORR. In order to incentivize authors to publish ORR and readers to use it, the work presented in this thesis also demonstrates benefits that go beyond reproducibility. The third issue refers to how research results are presented to other scientists. This work tackles this issue by presenting conceptual and technical solutions to create interactive geoscientific publications based on open reproducible research and (executable) research compendia.

\subsection{Scope and research questions}

This section defines the scope of this thesis and introduces the four research questions (RQ1-RQ4).

\subsubsection{Scope}

The work presented in this thesis focuses on publications from the geoscientific domain with readily available materials reporting on computational analyses of quantitative data, and on figures showing computational results produced by code and data. To keep the scope of this work manageable, only computational results based on $R$ (R Core Team, 2018), a programming language for statistics, are considered. To catch current practice, the paper corpora used in this work contain geoscientific articles published between 2010 and 2017. This thesis does not consider concepts similar to reproducibility, such as replicability which is about the attempt to confirm results by independent experiments (Bollen et al., 2015). Furthermore, scientific outcomes not based on source code, e.g. papers describing concepts or results based on qualitative research (e.g. interviews), are excluded. The reproducibility of such results is beyond this thesis' scope. Next, this work also does not consider papers about (geographic) information systems and new software frameworks or architectures. In terms of figures, this dissertation does not consider visualizations that show photographs, processes, or diagrams based on ready-to-use software.

\subsubsection{Research questions}

This thesis is structured according to the following research questions (RQs).

RQ1: What is the status quo of open reproducible research in the computational geosciences? The goal of this question is to reveal cultural and technical obstacles which prevent geoscientists from publishing open reproducible research 
and which impede readers' attempts to reproduce computational research results. Furthermore, this question aims at assessing whether existing geoscientific articles are reproducible. The results also inform RQ2.

RQ2: How can we assist geoscientists in publishing open reproducible research?

In order to tackle the barriers that prevent the realization of ORR (RQ1), this research question aims at identifying concrete guidelines and strategies for authors who aim at publishing ORR. ORR is not only needed to fulfill high scientific standards but also to realize the idea of an interactive geoscientific paper as mentioned in the introductory chapter. Otherwise, it is not possible to provide tools for inspecting the materials employed and for re-running a computational analysis with a modified parameter or another dataset.

RQ3: Which concrete incentives are provided by open reproducible research beyond re-computable results?

Being able to re-compute the results reported in a paper is a crucial benefit of ORR. However, if code and data are available, further opportunities exist which add to a researcher's workflow while studying a research paper. These opportunities are needed for motivating authors to publish their research in an open and reproducible way. The realization of selected opportunities is part of RQ4.

RQ4: How can we link code, data, and a specific computational result to create interactive geoscientific papers?

Even if a paper comes with reproducible code and data, the text is disconnected from the underlying source code and data. Understanding how the analyst computed a specific computational result (e.g. a figure or number) consequently requires time and effort from reviewers and readers. The goal of this question is to describe and implement an approach to link the three components paper, source code, and datasets. These connections might assist readers in accessing and reusing source code and data subsets that were used to compute a specific computational result in a paper. 


\subsection{Methodology}

This section provides an overview of the methods used to answer the research questions introduced in the previous section (see Figure 1.1 for an overview of research questions, methods, and contributions). The five complementary methods enabled collecting quantitative and qualitative data.

\section{Online surveys}

In order to obtain an initial overview of open reproducible research in the computational geosciences, this thesis reports on two separate online surveys with geoscientists. According to Lazar et al. (2017), running surveys is an efficient means to generate answers from many people. The collected data was analyzed with the help of descriptive statistics. In the first survey $(n=146)$, geoscientists who participated in the $E G U^{1}$ conference were asked about the availability of code and data underlying the results reported in their papers. They were also asked for needs when working with open reproducible research considering the perspective of a reader. However, one survey cannot cover all topics related to reproducibility, but it is also interesting to examine the reasons behind unreproducible papers. To investigate these issues, the second survey addressed the authors who contributed to the AGILE conference and referred to their paper directly. The authors were asked for concrete barriers that prevented them from publishing the materials underlying the results in their paper. The findings from the two online surveys thus contributed to describing the status quo of reproducibility in the geosciences (RQ1) and identifying incentives to publish reproducibly (RQ3). However, reproducibility has proven to be a complex topic which is difficult to capture solely with surveys including predefined questions. In order to obtain more profound knowledge, we thus decided on interviewing geoscientists personally.

\section{Semi-structured interviews}

In contrast to surveys, interviews allow participants to explain their thoughts in more detail (Lazar et al., 2017). In addition, the interviewer can ask further questions if an answer is unclear or of particular interest. Hence, this approach is a suitable means to investigate the issues identified in the survey more deeply. By considering the perspectives of authors and readers, the interviews investigated obstacles which impeded open reproducible research in past publications. Moreover, the interviewees could describe how they usually study an article and, related to their current workflow, mention needs, requests, and ideas when working with ORR.

\footnotetext{
${ }^{1}$ European Geosciences Union General Assembly: https://www.egu2016.eu/, last access: 01.04.2019.
} 
The findings from the semi-structured interviews deepened the insights from the two surveys mentioned above and thus also contributed to RQ1 and RQ3. Nine semi-structured interviews with researchers from the geoscientific domain were conducted. The responses to the interview questions were analyzed with the help of the grounded theory approach (Glaser and Strauss, 1967).

\section{Focus group}

Open science in general and, in particular, open reproducible research are interdisciplinary topics relevant to researchers from different scientific (sub-)disciplines. A focus group can bring participants from different areas together to collect qualitative data efficiently in one session (Lazar et al., 2017). In order to complement the semi-structured interviews, this thesis also reports on a focus group session with five additional geoscientists. In contrast to interviews, a focus group provides space for a discussion between participants which might elicit different ideas and opinions compared to those during the one-on-one interviews. The discussion included the same guiding questions as during the interviews, and the data analysis followed the same grounded theory approach. Hence, the focus group also helped to answer RQ1 and RQ3.

The three approaches described so far revealed cultural obstacles that prevented geoscientists from publishing and using ORR. Despite these issues, it would be interesting to check whether papers that have code and data attached are, in fact, reproducible or if technical issues impede successful execution of the analysis. For this reason, we conducted reproducibility studies to complement the qualitative data with quantitative numbers.

\section{Reproducibility studies}

The description of the current state of open reproducible research in the computational geosciences (RQ1) also requires a thorough analysis of existing geoscientific articles. In addition to the subjective feedback of the study participants, this thesis reports on two reproducibility studies. The first study assessed the availability of materials (e.g. code and data) underlying the results reported in 31 scientific articles submitted to the $A G I L E^{2}$ conference. For this purpose, the analysis considered data, methods, and results for each paper separately. These three paper components were then assigned to one of the four categories unavailable, documented, available, and permanently available. The second study comprised a sample of 41 open access articles submitted to Copernicus Publications and Journal of statistical software. These

\footnotetext{
${ }^{2}$ Association of Geographic Information Laboratories for Europe: https : //agile-online . org/, last access: 01.04.2019.
} 
papers had $R$ code and data attached making it possible to re-run the analysis and to note technical issues that impeded the execution of the scripts. Also, the analysis included a comparison of the figures produced by the code with the original ones from the article to find differences concerning the actual results and the design of the figures. Besides an initial assessment of whether geoscientific papers are reproducible, a further output of this approach was a set of executable research articles. These could be used for proposing and demonstrating strategies to overcome reproducibility issues (RQ2) as well as for developing a concept to connect the paper with the source code and the data (RQ4).

\section{Workshops with experts}

The idea to assist authors in publishing ORR and to transform static scientific articles into interactive papers has implications for different stakeholders across academia. Consequently, two workshops were organized with experts from research, the publishing sector, and the university library. Both workshops included open discussions about the developed strategies and concepts. In order to support the discussion, mock-ups and prototypes were prepared to demonstrate how executable and interactive papers could be created by authors and used by readers. Based on these means, the stakeholders could express concerns, provide feedback, and give advice while considering their individual perspective. Their comments were used to update the concepts iteratively. The workshops helped to answer RQ2 and RQ4.

All materials from the studies (interview and survey questions, source code, datasets) can be accessed online (see supplemental material in section 10). The papers presented in chapter 3,5 , and 6 are also available as executable research compendia.

\subsection{Key contributions}

This thesis comprises the following four key contributions: (C1) obstacles that prevented geoscientists form publishing and using ORR, (C2) strategies to overcome these issues, (C3) incentives ORR provides for authors and readers, and (C4) opportunities to create interactive geoscientific papers.

Contribution No. 1 contains a set of obstacles which prevented geoscientists from publishing ORR and an initial assessment of whether geoscientific articles are reproducible. It addresses RQ1.

We found that geoscientists had a diverse understanding of the term "open reproducible research". Consistent with literature (cf. Goodman et al., 2016), there 
was confusion between the two related terms "reproducibility" and "replicability". Furthermore, we identified a number of obstacles that prevented authors from publishing ORR and that stood in the way of readers trying to work with ORR. First, the methodology section often did not describe the used procedure in sufficient detail. A further issue was a lack of openness. Authors feared to lose their competitive advantage when publishing their source code and data. Another reason was the effort and time required to prepare these materials. Next, the use of licensed software made it impossible to share it. Finally, assisting tools for publishing ORR were missing. Nevertheless, even if materials were accessible, re-running the computations was perceived as being too time-consuming.

The reproducibility studies showed that the majority of papers did not have source code and data attached. Most articles only provided documentation of the used methods making it impossible for readers and reviewers to directly reproduce the results and to reuse the materials. However, publicly accessible source code and data did not guarantee reproducible research. The execution of the source code scripts attached to 41 additional articles revealed technical issues of different complexity: minor issues required to install a library; substantial issues emerged, for example, because of wrong file directories; severe issues required a more profound understanding of the source code to solve, for example, a flawed functionality; system-dependent issues occurred due to the use of a different operating system. Moreover, the computational outputs, i.e. the figures, did not match those from the article. The differences were related to the design of the figures (e.g. different colors, scale) and the actual numbers.

Contribution No. 2 comprises a set of recommendations and strategies to overcome the issues listed above. It refers to RQ2.

First, authors should use free open source tools instead of licensed software to enable reproduction by readers who do not own a license. Next, ORR should be part already of study courses in university to address early career scientists. With this approach, the students might consider "open science" practices for their first scientific contributions and thus reap the benefits from the beginning. In addition, journals and conferences should establish author guidelines which also consider reproducibility. On top of that, "open science" badges could increase the visibility of open and reproducible articles. Finally, authors should make sure the supplemented scripts are not only accessible but also executable and thus reusable, for example, by using Docker and executable documents, such as RMarkdown.

To facilitate the publication of re-computable research results, this thesis proposes a standardized packaging mechanism called "executable research compendium" (ERC). It builds upon the concept of a research compendium as mentioned in the 
introductory chapter. An ERC encapsulates all materials required to reproduce the computational results reported in a scientific article in a Docker container. Besides the actual paper, an ERC contains the dataset, the source code including configurations, and the software environment. The design of the ERC considers the needs of several stakeholders. For example, authors need support to create an ERC and readers want to re-run the computations with a single click (see also "one-click-reproduce" by Pebesma (2013)). Furthermore, reviewers would like to inspect the underlying research components to assess the validity of the results before publication. To serve the needs of authors, readers, reviewers, and publishers, this work describes a five-step publication process including submission, validation, review, publication, and reuse of executable research compendia. Since an ERC contains all materials in a reproducible way, it is a fundamental requirement for the realization of interactive geoscientific papers as proposed in the following two contributions.

Contribution No. 3 includes the description and implementation of an extended workflow for studying a research article. The workflow encapsulates the benefits of ORR and ERCs for reviewers, readers, and authors. It corresponds to RQ3.

We found that readers can benefit from ORR and ERCs at different stages of their research workflow while studying an article. First, readers can discover papers with the help of fine-grained queries by also considering information included in the code (e.g. the used functions) or in the data (e.g. the spatial or temporal information). Second, ORR allows them to inspect the code and the data underlying the numbers, tables, and figures to understand how the authors achieved these results. Next, they can manipulate single parameters in the code underlying a figure and then observe how different parameter values affect the final outcome. Then, readers can substitute the underlying dataset for seeing if the analysis is applicable to another dataset, for example, of a different region. Finally, we identified compare as a cross-cutting task which is relevant in each step of the workflow, for example, before and after manipulating parameters. Each of these workflow steps potentially contributes to readers' understanding making it easier for them to reuse the materials and to build upon the work. Consequently, the workflow based on ERCs also involves incentives for authors. They can make their work better discoverable and understandable, both essential drivers for receiving citations. Facilitating others to build upon their work might also result in future collaborations. To demonstrate the realizability of the workflow steps (discover, inspect, manipulate, substitute, compare), we implemented a prototypical web application.

Contribution No. 4 comprises the concept, implementation, and initial evaluation of bindings, i.e. fine-grained links between those parts of the source code and the dataset that produced a specific computational result in the article (RQ4). Bindings 
are part of executable research compendia and can be used to create interactive figures.

Bindings help to realize the workflow steps described in the previous contribution and are defined by the author of the paper while creating an ERC. Readers and reviewers can use bindings for a detailed inspection of how the author achieved a specific result. In order to create figures that can be manipulated, the code component specified in a binding can also explicitly refer to individual parameters which are part of the analysis. This reference makes it possible for authors to attach UI widgets (e.g. a slider) to these parameters which change the initial value. In addition, the description of the used data subset makes it easier for readers to create a compatible dataset to substitute the original one. Finally, readers can easily reuse the code snippets and data subsets specified in a binding for their research, thereby giving credit to the original authors.

To showcase the technical feasibility of the bindings concept, we implemented it as part of the web application that demonstrated the ERC-based workflow. First, the application allows authors to specify a binding by selecting the computational result, the relevant code lines, the data subset, and a UI widget. Based on this information, it is possible to create an interactive figure for reviewers and readers. For example, while reading an article, readers can inspect those code lines and data subsets that produced a specific figure. Furthermore, they can manipulate the parameters and observe changes in the results.

Finally, we evaluated the bindings concept and its implementation by creating bindings for figures in existing scientific articles. We found that many figures were based on parameters that could be manipulated by using a slider or radio buttons. Several of the examined articles from the reproducibility study contained concrete suggestions for changing parameter values. This finding shows there is a need for means that allow others to change the initial values set by the author, for example, with the help of interactive figures. This interaction is, however, difficult to achieve with traditional publications, such as static PDFs. We also provided initial measures to assess the effort required from authors to create bindings and the time readers need for using the resulting interactive figure.

In summary, the key contributions of this dissertation are fourfold: First, it describes the status quo of reproducibility in the computational geosciences. Second, this work recommends technical and cultural solutions to the identified obstacles. Next, it identifies the benefits of open and executable research for authors and readers. Finally, this thesis demonstrates the opportunity to create interactive geoscientific papers based on ORR and ERCs. These contributions can help to increase the number of reproducible papers and reap the advantages of attached code and data. 


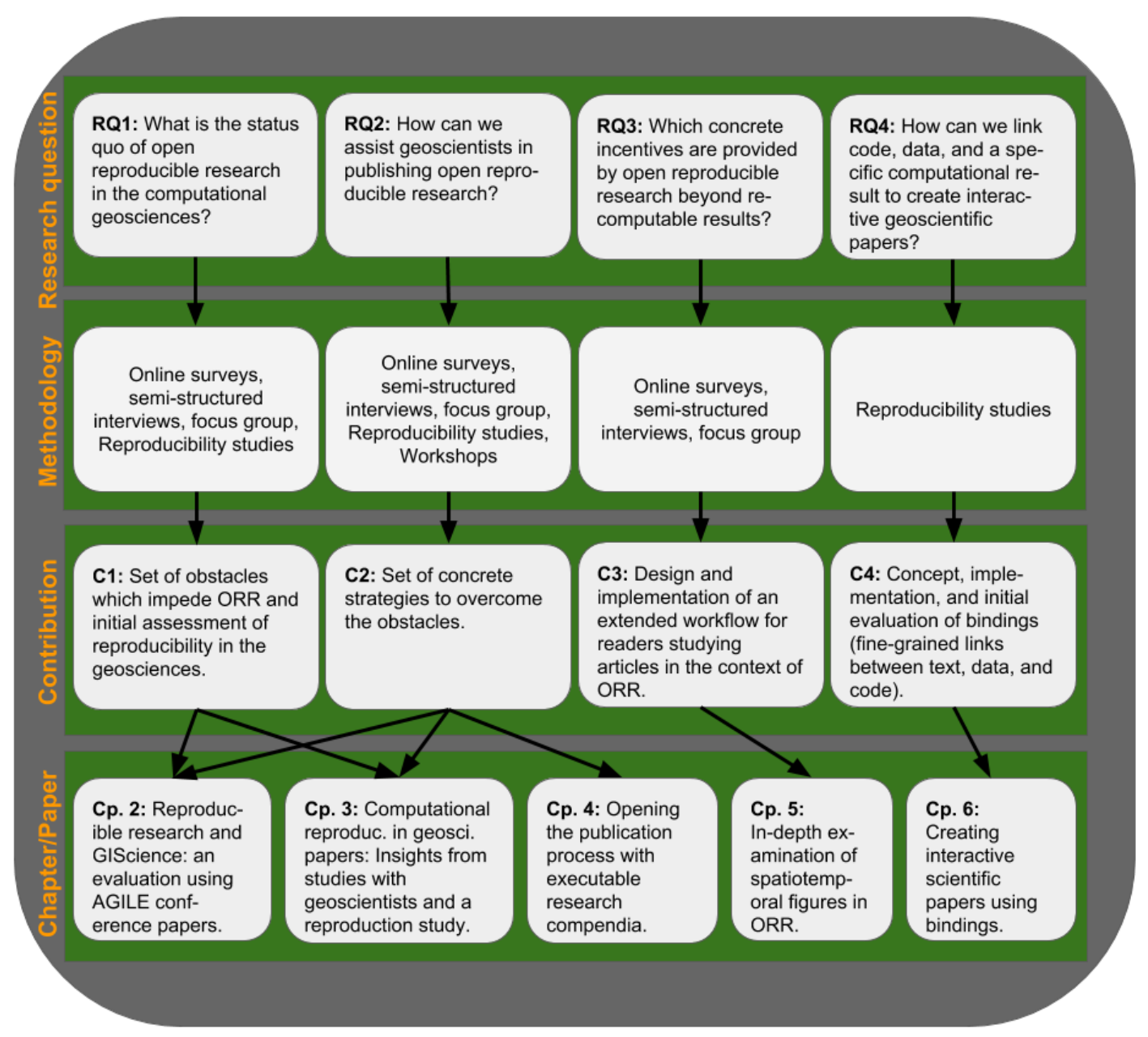

Fig. 1.1: Overview of the methods employed to answer the research questions RQ1 to RQ4, the resulting contributions $\mathbf{C} \mathbf{1}$ to $\mathbf{C} 4$, and the corresponding papers and chapters.

\subsection{Thesis outline}

The remainder of this dissertation is structured as follows: Chapter 2 contains the reproducibility study with papers submitted to the AGILE conference. Afterward, chapter 3 comprises technical and cultural obstacles which impede open reproducible research in the geosciences considering the perspective of authors and readers. The concept of an executable research compendium and its integration into the publication process is part of chapter 4. Next, incentives to publish ORR that go beyond reproducibility are described in chapter 5. Subsequently, the concept, implementation, and evaluation of bindings are presented in chapter 6 . In chapter 7 , the overall findings of the thesis are discussed considering the four research questions, the generalizability of the results, and limitations. The dissertation concludes by summarizing key insights in chapter 8 and promising directions for future work in chapter 9. 


\title{
Reproducible research and GIScience: an evaluation using AGILE conference papers
}

\author{
This chapter was published as: Nüst, Daniel, Carlos Granell, Barbara Hofer, Markus \\ Konkol, Frank O. Ostermann, Rusne Sileryte, and Valentina Cerutti (2018). "Repro- \\ ducible research and GIScience: an evaluation using AGILE conference papers". In: \\ PeerJ, 6:e5072. eprint: https://doi .org/10.7717/peerj .5072. It corresponds to \\ P2 in the list of publications.
}

\begin{abstract}
The demand for reproducible research is on the rise in disciplines concerned with data analysis and computational methods. Therefore, we reviewed current recommendations for reproducible research and translated them into criteria for assessing the reproducibility of articles in the field of geographic information science (GIScience). Using these criteria, we assessed a sample of GIScience studies from the Association of Geographic Information Laboratories in Europe (AGILE) conference series, and we collected feedback about the assessment from the study authors. Results from the author feedback indicate that although authors support the concept of performing reproducible research, the incentives for doing this in practice are too small. Therefore, we propose concrete actions for individual researchers and the GIScience conference series to improve transparency and reproducibility. For example, to support researchers in producing reproducible work, the GIScience conference series could offer awards and paper badges, provide author guidelines for computational research, and publish articles in open access formats.
\end{abstract}

\subsection{Introduction}

A "reproducibility crisis" has been observed and discussed in several scientific disciplines such as economics (Ioannidis et al., 2017), medical chemistry (Baker, 2017), neuroscience (Button et al., 2013), and for scientific studies in general, across various disciplines (Ioannidis, 2005). The lack of reproducibility in scientific studies stems from researchers facing challenges in understanding and re-creating others' results, a situation that is common in data-driven and algorithm-based research. However, even though algorithms are becoming more relevant in GIScience, a reproducibility 
crisis has not yet been observed in this field. In GIScience, failures to reproduce are not yet a topic of broad and common interest, but this field should be working to prevent a crisis instead of reacting to one. Given this motivation, we aim to adopt the observations and challenges of reproducible research from other disciplines to the GIScience community. Then, we use these adapted criteria to assess the reproducibility of research produced by members of this field and presented at a conference for the Association of Geographic Information Laboratories in Europe (AGILE), which has organized annual conferences on GIScience topics since $1998^{1}$. The conference series's broad topical scope and its notoriety in the GIScience community make it a reasonable starting point to investigate the level of reproducibility in GIScience research. This publication continues a collaboration started at the AGILE 2017 pre-conference workshop "Reproducible Geosciences Discussion Forum" 2 .

In this work, we first review papers from other disciplines, which provide recommendations on how to make research more transparent and reproducible. This literature study provides the general criteria we used to systematically evaluate a sample of 32 AGILE conference papers from the last eight years. From this evaluation and the lessons learned by others, we formulate recommendations for the AGILE community, ranging from individual researchers' practices to practices to be carried out by conference organizations. Because of its international reach, the broad range of topics, and long-sustained community, we argue that AGILE is in a unique position to take a leading role to promote reproducibility in GIScience. The following research questions (RQs) structure the remainder of this article:

- RQ 1: What are the general criteria for reproducible research?

- RQ 2: What are the key criteria for reproducible research in GIScience?

- RQ 3: How do AGILE conference papers meet these reproducibility criteria?

- RQ 4: What strategies could improve reproducibility in AGILE contributions and GIScience in general?

"Related Work" provides references targeting RQ 1, which are detailed further in "Assessment of Reproducibility" to address RQ 2. The results of applying the criteria ("Results") answer RQ 3, and the discussion ("Discussion") responds to RQ 4.

\footnotetext{
${ }^{1}$ Past AGILE conferences: https://agile-online.org/index.php/conference/ past-agile-conferences, last access for this and the following URL: 01.04.2019.

${ }^{2}$ Workshop report: http://o2r.info/reproducible-agile/2017/
} 


\subsection{Related Work}

Reproducible research is a frequently discussed topic in editorials and opinion articles in high-impact journals (cf. section 2.3.2 "Recommendations and suggestions in literature"). Extensive studies on the state of reproducibility have been conducted in some domains, e.g. in computer systems research (Collberg and Proebsting, 2016, see also their project website ${ }^{3}$ ) or bioinformatics (Hothorn and Leisch, 2011). For the field of geoscience research, some discussion of reproducibility has happened sporadically for quantitative geography (Brunsdon, 2016), cartography (Giraud and Lambert, 2017), and volunteered geographic information (VGI) (Ostermann and Granell, 2017), but no comprehensive study of reproducibility in the GIScience domain has been conducted.

Even though recent studies highlight an increased awareness of and willingness for open research, they also draw attention to persistent issues and perceived risks associated with data sharing and publication, such as the lack of rewards and the risk of losing recognition in a competitive academic environment (Tenopir et al., 2011; Ioannidis, 2014). Beyond individual concerns, there are systematic impediments. Some studies have mentioned that reproducible research is not in the individual researcher's domain but instead is a multi-actor endeavor, which requires a collective mind shift within the scientific community (Stodden et al., 2016; McNutt, 2014; Ioannidis, 2014). Funding agencies, research institutions, publishers, journals, and conferences are all responsible for promoting reproducible research practices. Existing examples (journals welcoming reproducible papers: Information Systems ${ }^{4}$, Vadose Zone Journal ${ }^{5}$, GigaScience ${ }^{6}, J A S A^{7}$ ) are remarkable, yet in general, they are scarce and testimonial.

Another hindrance to reproducible research is that, given the distinct nature and variety of research practices, the term reproducibility has been used with varying meanings and may stand for repeatability, robustness, reliability, or generalizability of scientific results (Nature Editorial, 2016). There has been some confusion about contradictory meanings in the literature (see for example Mark Liberman's "Replicability vs. reproducibility" ${ }^{\prime 8}$ ). Wikipedia's definition ${ }^{9}$ is widely used to distinguish both terms:

\footnotetext{
${ }^{3}$ Project website: http://reproducibility.cs.arizona.edu/, last access for this and the following URLs: 01.04.2019.

${ }^{4}$ Information Systems: https ://www.elsevier.com/journals/information-systems/0306-4379

${ }^{5}$ Vadose Zone Journal: https://dl . sciencesocieties .org/publications/vzj/articles/14/10/ vzj2015.06.0088

${ }^{6}$ GigaScience: https://academic.oup.com/gigascience/pages/instructions_to_authors

${ }^{7}$ JASA: http://www.sph.umn.edu/news/wolfson-named-reproducibility-editor-asa-statistics-journal

${ }^{8}$ Replicability vs. reproducibility: http://languagelog.ldc .upenn. edu/nll/?p=21956

${ }^{9}$ Wikipedia's definition: https://en.wikipedia.org/wiki/Reproducibility
} 
Reproducibility is the ability to get the same research results using the raw data and computer programs provided by the researchers. A related concept is replicability, meaning the ability to independently achieve similar conclusions when differences in sampling, research procedures and data analysis methods may exist.

Leek and Peng (2015) similarly define reproducibility as the ability to compute precisely the same results of a study based on original input data and details of the analysis workflow. They refer to replicability as obtaining similar conclusions about a research question derived from an independent study or experiment. A Nature Editorial (2016) defines reproducibility as achieved when "another scientist using the same methods gets similar results and can draw the same conclusions". Stodden et al. (2016) base their reproducibility enhancement principles on "the ability to re-run the same computational steps on the same data the original authors used". While most statements in the literature show that researchers have a common understanding of what these two concepts mean, the interpretation and application of these concepts by the scientific communities are still inconsistent and leads to different methods and conventions for disseminating scientific work. In the field of GIScience, Ostermann and Granell (2017) argue that "reproduction is always an exact copy or duplicate, with the same features and scale, while a replication resembles the original but allows for variations in scale for example". Hence, reproducibility is exact whereas replicability means confirming the original conclusions, though not necessarily with the same input data, methods, or results.

\subsection{Materials and Methods}

\subsubsection{The paper corpus}

We consider the AGILE conference series publications to be a representative sample of GIScience research because of the conferences' broad topical scope. Since 2007, the AGILE conference has had a full paper track (cf. Pundt and Toppen, 2017) and a short paper track with blind peer review. The latter is published for free on the AGILE website. Legal issues (full paper copyrights lie with the publisher Springer ${ }^{10}$ ), and practical considerations (assessment of reproducibility is a manual, time-consuming process; old publications introduce bias because of software unavailability) led us to choose to apply our evaluation only to nominees for the "best full and short paper" awards for 2010, and 2012 to 2017 (no records for the best paper award could be found for 2011). Typically, there are three full paper and two short paper candidates

\footnotetext{
${ }^{10}$ Springer series on Geoinformation and Cartography: https://agile-online.org/index.php/ conference/springer-series, last access: 01.04.2019.
} 
per year ${ }^{11}$. Exceptions are 2013 with only two full papers and 2010 without any short papers. The corpus contains 32 documents: 20 full papers (7.9\% of 253 full papers since 2007) and 12 short papers ${ }^{12}$.

An exploratory text analysis of the paper corpus investigated the occurrence of keywords related to reproducibility, data, and software. The code is published as an executable document ${ }^{13}$ (cf. Nüst, 2018). Figure 2.1 illustrates the most frequent terms mentioned. Table 2.1 shows keyword occurrence per paper and in the entire corpus (bottom row "Total"). Keyword identification uses word stems, e.g. "reproduc" includes "reproducible", "reproduce", and "reproduction" (see Nüst (2018) for details). While this matches common and established (technical) terms, it might not capture all phrases an author could use to describe reproducibilityrelated aspects of the work. Putting these corner cases aside, the numbers are clear enough to draw the following conclusions. Few papers mention reproducibility, some mention code and software, and many mention processes, algorithms, and data. This points to data and analysis are generally discussed in the publications while being able to re-create the data and analyses is not deliberated.

\subsubsection{Assessment of reproducibility}

\section{Recommendations and suggestions in literature}

Scientists from various disciplines suggest guidelines for open and reproducible research considering the specific characteristics of their field, e.g. Sandve et al. (2013) for life sciences, McNutt (2014) for field sciences, and Gil et al. (2016) for the geoscience paper of the future. Our goal was first to identify common recommendations that are applicable across research fields, including GIScience.

Suggested guidelines found in the reproducibility-related papers we investigated were categorized according to four aspects: data concerns all inputs; methods cover everything on the analysis of data, e.g. algorithms, parameters, and source code; results include (intermediate) data and parameters as well as outcomes such as statistics, maps, figures, or new datasets; and structure considers the organization and integration of the other aspects. While some of the publications focus on specific aspects such as data (Gewin, 2016), code (Stodden and Miguez, 2014), workflow semantics (Scheider et al., 2017), and results (Sandve et al., 2013), others provide an all-embracing set of research instructions (Stodden et al., 2016; Nosek et al., 2015; Gil et al., 2016).

\footnotetext{
${ }^{11}$ Conference proceedings: https://agile-online.org/index.php/conference/proceedings, last access for this and the following URLs: 01.04.2019.

${ }^{12}$ Full number of short papers cannot be derived automatically (cf. Nüst, 2018).

${ }^{13}$ Using R Markdown, see http://rmarkdown.rstudio.com/.
} 
Tab. 2.1: Reproducibility-related keywords in the corpus, ordered by sum of matches per paper. For full references of the corpus papers, see supplemental material under https://doi.org/10.7717/peerj.5072/supp-1 (last access: 01.04.2019).

\begin{tabular}{|c|c|c|c|c|c|c|c|c|c|c|}
\hline Citation & $\begin{array}{l}\text { Repro- } \\
\text { duc. }\end{array}$ & Replic. & $\begin{array}{l}\text { Rep- } \\
\text { eatab. }\end{array}$ & Code & Software & $\begin{array}{c}\text { Algo- } \\
\text { rithm(s) }\end{array}$ & $\begin{array}{c}\text { (pre)- } \\
\text { process }\end{array}$ & Data & Result(s) & All \\
\hline Foerster et al. (2012) & 0 & 0 & 0 & 2 & 3 & 11 & 140 & 129 & 41 & 326 \\
\hline Wiemann \& Bernard (2014) & 0 & 0 & 0 & 0 & 0 & 0 & 20 & 98 & 3 & 123 \\
\hline Mazimpaka \& Timpf (2015) & 0 & 0 & 0 & 3 & 0 & 4 & 4 & 97 & 10 & 118 \\
\hline Steuer et al. (2015) & 0 & 0 & 0 & 0 & 0 & 25 & 12 & 64 & 17 & 118 \\
\hline Schäffer et al. (2010) & 0 & 0 & 0 & 0 & 10 & 1 & 26 & 65 & 6 & 108 \\
\hline Rosser et al. (2016) & 0 & 0 & 0 & 0 & 2 & 1 & 42 & 51 & 6 & 105 \\
\hline Gröchening et al. (2014) & 0 & 0 & 0 & 0 & 0 & 3 & 2 & 69 & 27 & 101 \\
\hline Almer et al. (2016) & 0 & 0 & 0 & 1 & 1 & 1 & 22 & 53 & 22 & 100 \\
\hline Magalhães et al. (2012) & 0 & 0 & 0 & 2 & 1 & 20 & 52 & 9 & 1 & 85 \\
\hline Juhász \& Hochmair (2016) & 0 & 0 & 0 & 0 & 1 & 1 & 2 & 55 & 11 & 70 \\
\hline Wiemann (2016) & 0 & 0 & 0 & 0 & 3 & 0 & 8 & 55 & 1 & 69 \\
\hline Fan et al. (2014) & 0 & 0 & 0 & 0 & 0 & 3 & 8 & 44 & 12 & 67 \\
\hline Merki \& Laube (2012) & 0 & 0 & 0 & 0 & 0 & 9 & 6 & 40 & 6 & 62 \\
\hline Zhu et al. (2017) & 2 & 2 & 0 & 2 & 0 & 10 & 7 & 32 & 6 & 61 \\
\hline Kuhn \& Ballatore (2015) & 0 & 0 & 1 & 2 & 14 & 1 & 5 & 26 & 8 & 58 \\
\hline Soleymani et al. (2014) & 1 & 0 & 0 & 0 & 0 & 0 & 4 & 39 & 9 & 56 \\
\hline Fogliaroni \& Hobel (2015) & 0 & 0 & 0 & 0 & 0 & 3 & 14 & 30 & 5 & 52 \\
\hline Osaragi \& Hoshino (2012) & 0 & 0 & 0 & 0 & 0 & 0 & 5 & 36 & 7 & 48 \\
\hline Stein \& Schlieder (2013) & 0 & 0 & 0 & 0 & 0 & 0 & 3 & 42 & 3 & 48 \\
\hline Körner et al. (2010) & 0 & 0 & 0 & 0 & 0 & 6 & 5 & 30 & 4 & 45 \\
\hline Knoth et al. (2017) & 0 & 0 & 0 & 3 & 2 & 1 & 6 & 25 & 7 & 44 \\
\hline Raubal \& Winter (2010) & 0 & 0 & 0 & 1 & 1 & 1 & 18 & 0 & 13 & 34 \\
\hline Konkol et al. (2017) & 1 & 0 & 0 & 3 & 1 & 1 & 2 & 4 & 19 & 31 \\
\hline Kiefer et al. (2012) & 1 & 0 & 0 & 0 & 2 & 1 & 9 & 10 & 8 & 31 \\
\hline Haumann et al. (2017) & 0 & 0 & 0 & 0 & 0 & 6 & 8 & 10 & 2 & 26 \\
\hline Josselin et al. (2016) & 0 & 0 & 0 & 0 & 2 & 1 & 9 & 5 & 8 & 25 \\
\hline Heinz \& Schlieder (2015) & 1 & 0 & 0 & 2 & 1 & 3 & 2 & 14 & 2 & 25 \\
\hline Osaragi \& Tsuda (2013) & 0 & 0 & 0 & 1 & 1 & 0 & 3 & 16 & 2 & 23 \\
\hline Baglatzi \& Kuhn (2013) & 1 & 0 & 0 & 0 & 0 & 0 & 6 & 12 & 3 & 22 \\
\hline Scheider et al. (2014) & 0 & 0 & 0 & 0 & 1 & 0 & 0 & 13 & 4 & 19 \\
\hline Brinkhoff (2017) & 0 & 0 & 0 & 0 & 1 & 9 & 2 & 3 & 2 & 17 \\
\hline Schwering et al. (2013) & 0 & 0 & 0 & 0 & 0 & 4 & 2 & 3 & 5 & 14 \\
\hline Total & 7 & 2 & 1 & 22 & 47 & 126 & 454 & 1,179 & 280 & 2,131 \\
\hline
\end{tabular}




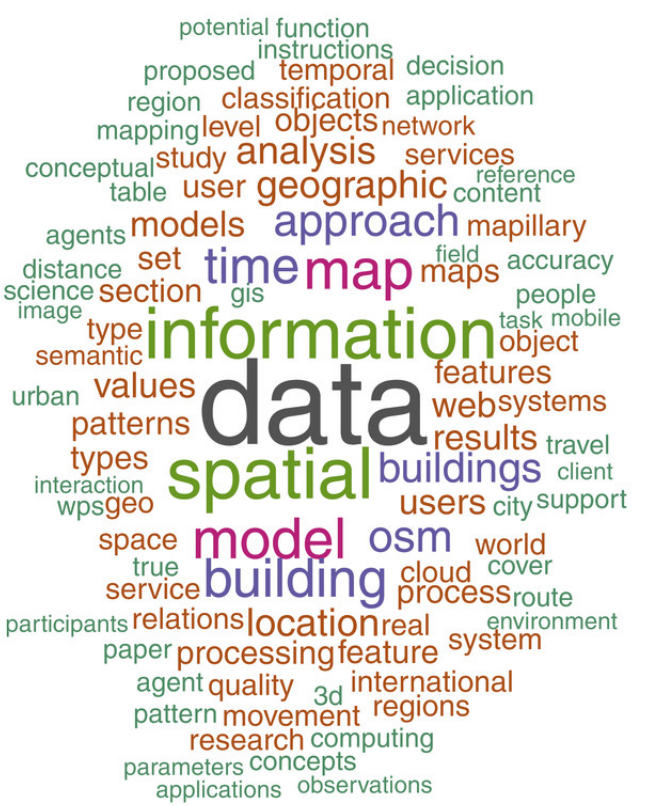

$\begin{array}{cccc}\text { place } & \text { word } & \mathbf{n} & \text { \# papers } \\ 1 & \text { data } & 1058 & 31 \\ 2 & \text { information } & 589 & 32 \\ 3 & \text { spatial } & 577 & 30 \\ 4 & \text { map } & 411 & 25 \\ 5 & \text { model } & 411 & 25 \\ 6 & \text { building } & 381 & 24 \\ 7 & \text { time } & 378 & 30 \\ 8 & \text { approach } & 297 & 32 \\ 9 & \text { osm } & 292 & 8 \\ 10 & \text { buildings } & 266 & 15 \\ 11 & \text { geographic } & 249 & 28 \\ 12 & \text { location } & 239 & 26 \\ 13 & \text { analysis } & 229 & 28 \\ 14 & \text { users } & 225 & 19 \\ 15 & \text { results } & 207 & 30 \\ 16 & \text { web } & 206 & 21 \\ 17 & \text { models } & 202 & 20 \\ 18 & \text { values } & 202 & 23 \\ 19 & \text { patterns } & 196 & 16 \\ 20 & \text { maps } & 189 & 20\end{array}$

Fig. 2.1: Two illustrations of the test corpus papers: word cloud, scaled and colored by the number of occurrence of words with at least 100 occurrences (96 unique words) (A); top words sorted by overall occurrence and number of papers including the word at least once (B).

Data. A recurring aspect we encountered is making data accessible for other researchers (cf. Reichman et al., 2011), ideally as archived assets having a Digital Object Identifier (DOI) and supplemented by structured metadata (Gewin, 2016). Stodden et al. (2016) consider legal aspects, such as sharing data publicly under an open license to clarify reusability. Further recommendations refer to modifying scientific practices, such as citation standards to ensure proper acknowledgment (Nosek et al., 2015), fostering data transparency (McNutt, 2014), and using open data formats to mitigate potentially disappearing proprietary software (Gewin, 2016). According to Reichman et al. (2011), journals and funders should include data sharing in their guidelines.

Methods. A key requirement (Sandve et al., 2013) concerning methods is sharing used or developed software, where software should be published using persistent links (Stodden et al., 2016; Gil et al., 2016) and descriptive metadata (Reichman et al., 2011). Similar to data, important concerns for software are open licensing (Barba, 2016) and proper credits (Stodden et al., 2016). Researchers can accomplish software transparency by using version control systems (cf. Sandve et al., 2013), and transparency mandates using open source instead of proprietary software (Steiniger and Hay, 2009). Since full computational reproducibility can depend on exact software versions (Gronenschild et al., 2012), the computational environment needs 
to be reported (Stodden et al., 2016; Gil et al., 2016). Further software-specific recommendations are workflow tracking (Stodden and Miguez, 2014) and keeping a record of analysis parameters (Gil et al., 2016). Sandve et al. (2013) suggest avoiding manual data manipulation steps and instead using scripts to increase transparency in data preprocessing.

Results. Sandve et al. (2013) focus on results-related guidelines such as storing intermediate results and noting seeds if computations include randomness. Journals should conduct a reproducibility check before publication (Stodden et al., 2016) or funding should be granted explicitly for making research results repeatable (Collberg and Proebsting, 2016). Finally, Barba (2016) describes the contents and benefits of a "reproducibility package" to preserve results.

Structure. While the papers discussed above focus on specific aspects of reproducibility, an overarching structure for all facets of research can provide valuable context. However, none of the suggestions for packaging workflows are widely established, for example, Gentleman and Lang (2007) use programming language packaging mechanisms, Bechhofer et al. (2013) Linked Data, or, as described in chapter 4 , nested containers.

Section summary. Most recommendations and suggestions to foster open and reproducible research address data and methods. Particularly, methods cover a broad range of aspects including recommendations on data preprocessing, the actual analysis, and the computational environment. Results receive less attention. One reason might be that they are strongly connected with other aspects. While most of the recommendations address authors, only a few target journals and research institutions.

\section{Definition and criteria}

This paper focuses on reproducibility in the context of conference publications and adopts the described consensus (see "Related work") for the following definition.

A reproducible paper ensures that a reviewer or reader can re-create the computational workflow of a study or experiment, including the prerequisite knowledge and the computational environment. The former implies the scientific argument to be understandable and sound. The latter requires a detailed description of used software and data, and both being openly available. 
We build on the recommendations from "Recommendations and suggestions in literature" (section 2.3.2) and differentiate data, methods, and results as separate dimensions of reproducibility. We conceptualized each reproducibility dimension as a criterion, and for each criterion, we developed levels of attained reproducibility. In order to increase the reproducibility of this study and improve inter-rater agreement, we created a corresponding rubric that explains the requirements. Together, the three criteria and their levels address specifics of GIScience research and allow for a fine-grained assessment of reproducibility.

However, early during the evaluation process, it became clear that the assessed corpus papers showed considerable variation in data, methods, and type of results. For example, data used during the reported studies varies from spatial data to qualitative results from surveys. Methods are particularly diverse, ranging from the application of spatial analysis operations to statistical approaches or simulations. Results include maps, formulas, models, or diagrams. Therefore, we decided to split the methods criterion into three sub-criteria addressing the distinct phases and respective software tools: data preprocessing, analysis methods and workflows, and computational environment. Following this change, we re-evaluated already assessed corpus papers.

Figure 2.2 shows the reproducibility criteria for each of the categories Data, Methods, and Results, and their levels. The levels are either not applicable (NA) or range from no (value of 0) to full (3) reproducibility. The level 0 unavailable means that there is insufficient documentation in the paper or the information is only available upon request (since this cannot be guaranteed and we could not check availability for all studies). The level 1 documented means that there is sufficient information to re-create at least parts of the study, but no concrete data or code or models. Level 2 available means that the required data and code is accessible. Finally, level 3 available, open and permanent, adds the requirement of unrestricted and sustainable access, e.g. through permanent links to open repositories containing data, relevant methods and workflows (such as software versions, hardware specifications, scripts), and all results (including intermediary ones or those not discussed in detail in the study). The Methods criteria do not include the "permanent" aspect, because there is no suitable single identifier to define the complex properties of code, libraries, and system environment, although a DOI may be used to identify all these items as a source or binary files collectively. Licensing is important for reproducibility, because only a clear license, which ideally is well-known and established, allows the use of copyrighted content. So in this sense "open" means "open enough for reproduction", but in practice, the used licenses should be fully open and allow modification and sharing beyond mere access and use ${ }^{14}$.

\footnotetext{
${ }^{14}$ cf. The Open Definition, https://opendefinition.org/: "Open data and content can be freely
} used, modified, and shared by anyone for any purpose", last access: 01.04.2019 


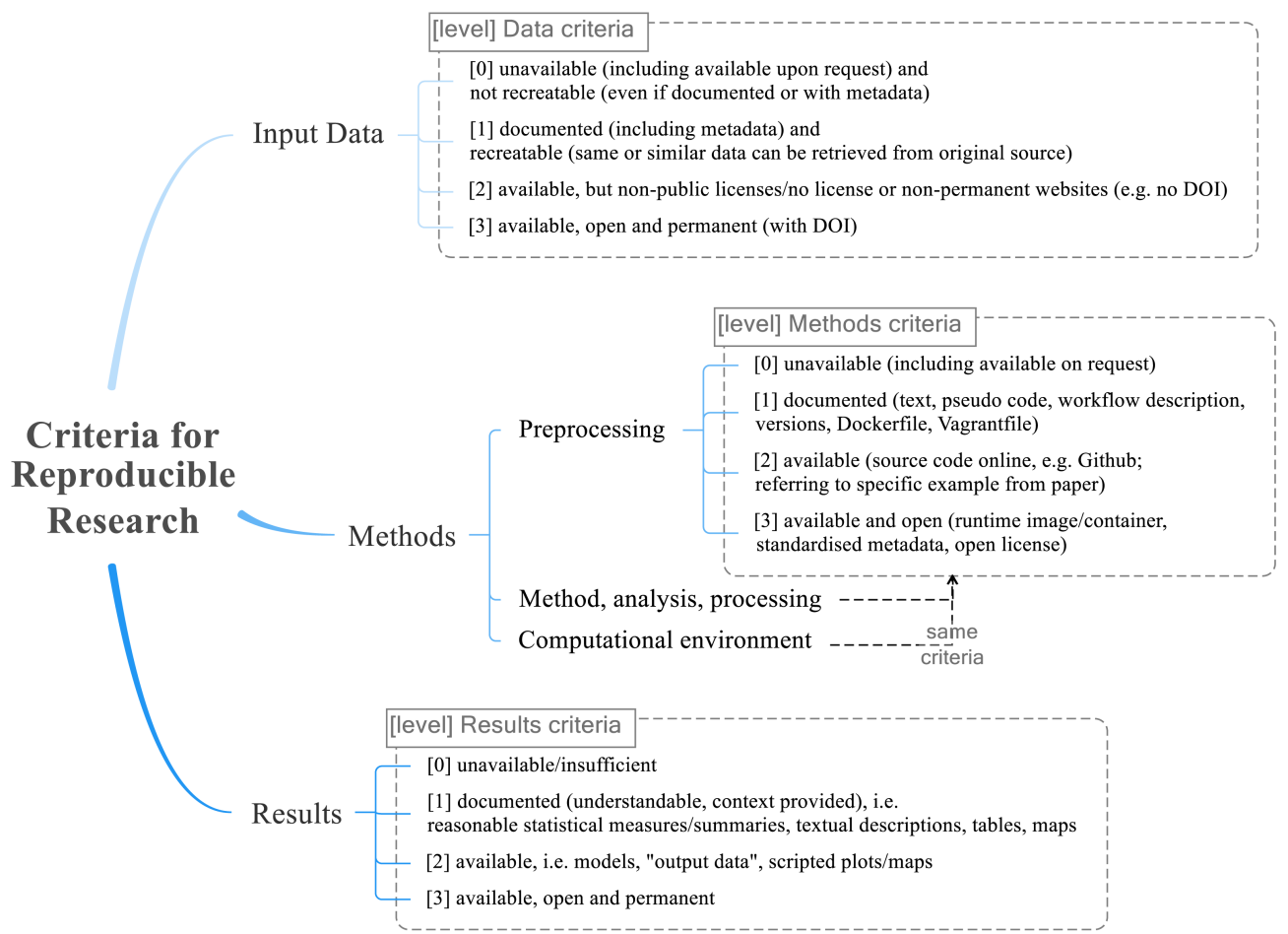

Fig. 2.2: The final reproducible research criteria used for the evaluation. The categories Data, Methods (sub-categories: preprocessing, method/analysis/processing, and computational environment), and Results each have four levels ranging from $0=$ not reproducible to $3=$ fully reproducible.

The intermediate levels ( 1 and 2 ) allow a differentiated evaluation. For example, for data at level 1, data is not accessible but documented sufficiently, so that others can re-create it. At level 2, data is available in a non-persistent way or with a restrictive license. The requirements are cumulative, meaning that higher levels of reproducibility include lower levels' requirements. The reproducibility rubric was developed in iterative discussions between all raters, using the examined literature on reproducibility as the point of reference.

By design, our criteria do not apply to conceptual research publications, namely those without data or code. Their evaluation is covered by an editorial peer review process (see for example Ferreira et al. (2015) for history and future of peer review), and assessing the merit of an argument is beyond the scope of this work.

\section{Author feedback on assessment of reproducibility (survey)}

To better understand the reasons behind the scores and to give the authors an opportunity to respond after the reproducibility of their research was assessed, we designed a survey using Google Forms ${ }^{15}$ (see Table 2.2, cf. Baker (2016a) for a large

\footnotetext{
${ }^{15}$ Google Forms: https://www.google.com/forms/about/, last access: 01.04.2019.
} 
scale survey on the topic). The full survey, as shown to the participants, is included in the supplemental material ${ }^{16}$.

Along with the survey, the authors received the results of our evaluation of their specific papers, and they were asked to express their agreement or disagreement with the results. The four main questions of the survey were designed to find out whether authors considered reproducibility important in the first place, and if so, what prevented them from fully achieving it. Finally, the authors were asked to provide their own opinion and suggestions for the AGILE community to encourage publishing fully reproducible papers.

\subsection{Results}

\subsubsection{Assessment of reproducibility}

To address RQ 3, we reviewed the papers in the corpus with the introduced criteria. Our objective in publishing the full evaluation results is not to criticize or rank individual papers, but to identify the current overall state of reproducibility in GIScience research reproducibly. The scientific merit of all papers was already proven by their nomination for the best paper award.

The procedure was as follows: First, we determined a maximum number of papers for a single evaluator to reach two evaluators per paper. Second, we grouped evaluators according to their affiliation or research group. Evaluators then chose to review papers without a conflict of interest on a first come first served basis until two goals were achieved: the evaluator reached her maximum number of reviews, and two evaluators from different research groups reviewed the paper. For assigning a level of reproducibility, the general guideline was to apply the lower of two possible levels in cases of doubt, such as partial fulfillment of a criterion or disagreement between the evaluators. All reviewers discussed disagreements and open questions after an initial round of evaluation comprising one to three reviews per researcher, and after completing all reviews. Because the assessment focuses on algorithmic and data-driven research papers, five fully conceptual papers were not assessed, while 15 partly conceptual ones were included. Notably, the data preprocessing criterion did not apply to 14 research papers. Table 2.3 shows the assessment's results.

Figure 2.3 shows the distribution of reproducibility levels for each criterion. None of the papers reach the highest level of reproducibility in any category. Only five papers reach level 2 in the data criterion, which is still the highest number of that

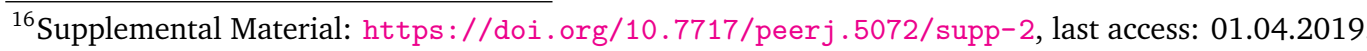


Tab. 2.2: Survey questions (except for paper identification questions; for full questionnaire see supplemental material under https://doi.org/10.7717/peerj.5072/ supp-2, last access: 01.04.2019).

\begin{tabular}{|c|c|}
\hline $\begin{array}{l}\text { 1. Have you considered the repro- } \\
\text { ducibility of research published in your } \\
\text { nominated paper? }\end{array}$ & $\begin{array}{l}\text { - Yes, it is important to me that my re- } \\
\text { search is fully reproducible } \\
\text { - Yes, I have somewhat considered repro- } \\
\text { ducibility } \\
\text { - No, I was not concerned with it } \\
\text { - Other (please add) }\end{array}$ \\
\hline $\begin{array}{l}\text { 2. Do you agree with our rating of your } \\
\text { publication? Please comment. }\end{array}$ & Open answer \\
\hline $\begin{array}{l}\text { 3a. Please rate how strongly the follow- } \\
\text { ing circumstances have hindered you } \\
\text { from providing all data, methods and } \\
\text { results used/developed during your re- } \\
\text { search? }\end{array}$ & $\begin{array}{l}\text { - The need to invest more time into the } \\
\text { publication } \\
\text { - Lack of knowledge how to include } \\
\text { data/methods/results into the publica- } \\
\text { tion } \\
\text { - Lack of tools that would help to attach } \\
\text { data/methods/results to the publication } \\
\text { - Lack of motivation or incentive } \\
\text { - Legal restrictions } \\
\text { Available ratings: } \\
\text { - Not at all } \\
\text { - Slightly hindered } \\
\text { - Moderately hindered } \\
\text { - Strongly hindered } \\
\text { - Main reason }\end{array}$ \\
\hline $\begin{array}{l}\text { 3b. Please add here if there were any } \\
\text { other hindering circumstances }\end{array}$ & Open answer \\
\hline $\begin{array}{l}\text { 4. What would you suggest to AG- } \\
\text { ILE community to encourage publishing } \\
\text { fully reproducible papers? }\end{array}$ & Open answer \\
\hline
\end{tabular}


Tab. 2.3: Reproducibility levels for paper corpus; "-" means category is not available. For full references of the corpus papers see https://doi.org/10.7717/peerj.5072/ supp-1 (last access: 01.04.2019).

\begin{tabular}{|c|c|c|c|c|c|c|}
\hline Author & Short paper & Input data & Preprocessing & $\begin{array}{c}\text { Method/ } \\
\text { Analysis/ } \\
\text { Processing }\end{array}$ & $\begin{array}{l}\text { Cmoputational } \\
\text { environment }\end{array}$ & Results \\
\hline Zhu et al. (2017) & & 0 & 1 & 1 & 1 & 1 \\
\hline Knoth et al. (2017) & & 0 & - & 0 & 1 & 1 \\
\hline Konkol et al. (2017) & & 2 & 2 & 1 & 1 & 1 \\
\hline Haumann et al. (2017) & $\mathrm{x}$ & 0 & 1 & 1 & 0 & 1 \\
\hline Brinkhoff (2017) & $\mathrm{x}$ & 0 & - & 1 & 0 & 0 \\
\hline Almer et al. (2016) & & 0 & - & 1 & 1 & 1 \\
\hline Wiemann (2016) & & 2 & - & 1 & 1 & 1 \\
\hline Juhász \& Hochmair (2016) & & 0 & 1 & 1 & 0 & 0 \\
\hline Josselin et al. (2016) & $\mathrm{x}$ & 1 & - & 0 & 0 & 1 \\
\hline Rosser et al. (2016) & $\mathrm{x}$ & 0 & - & 1 & 0 & 0 \\
\hline Kuhn \& Ballatore (2015) & & - & - & - & - & - \\
\hline Mazimpaka \& Timpf (2015) & & 2 & 1 & 1 & 1 & 1 \\
\hline Steuer et al. (2015) & & 2 & 0 & 1 & 1 & 1 \\
\hline Fogliaroni \& Hobel (2015) & $\mathrm{x}$ & - & - & - & - & - \\
\hline Heinz \& Schlieder (2015) & $\mathrm{x}$ & 0 & 0 & 1 & 1 & 1 \\
\hline Scheider et al. (2014) & & 1 & 1 & 2 & 1 & 1 \\
\hline Gröchening et al. (2014) & & 2 & 0 & 1 & 0 & 1 \\
\hline Fan et al. (2014) & & 0 & 1 & 1 & 0 & 1 \\
\hline Soleymani et al. (2014) & $\mathrm{x}$ & 0 & 0 & 1 & 0 & 0 \\
\hline Wiemann \& Bernard (2014) & $\mathrm{x}$ & 0 & 0 & 1 & 0 & 0 \\
\hline Osaragi \& Tsuda (2013) & & 0 & 1 & 1 & 0 & 1 \\
\hline Baglatzi \& Kuhn (2013) & & - & - & - & - & - \\
\hline Schwering et al. (2013) & $\mathrm{x}$ & 0 & 0 & 1 & - & 1 \\
\hline Stein \& Schlieder (2013) & $\mathrm{x}$ & 0 & - & 1 & 0 & 1 \\
\hline Osaragi \& Hoshino (2012) & & 0 & 0 & 1 & 0 & 1 \\
\hline Magalhães et al. (2012) & & 0 & 0 & 1 & 0 & 0 \\
\hline Foerster et al. (2012) & & 1 & - & 1 & 1 & 1 \\
\hline Merki \& Laube (2012) & $\mathrm{x}$ & 0 & - & 1 & 1 & 1 \\
\hline Kiefer et al. (2012) & $\mathrm{x}$ & 0 & 1 & 1 & 0 & 1 \\
\hline Raubal \& Winter (2010) & & - & - & - & - & - \\
\hline Schäffer et al. (2010) & & 0 & 0 & 1 & 1 & 1 \\
\hline Körner et al. (2010) & & - & - & - & - & - \\
\hline
\end{tabular}



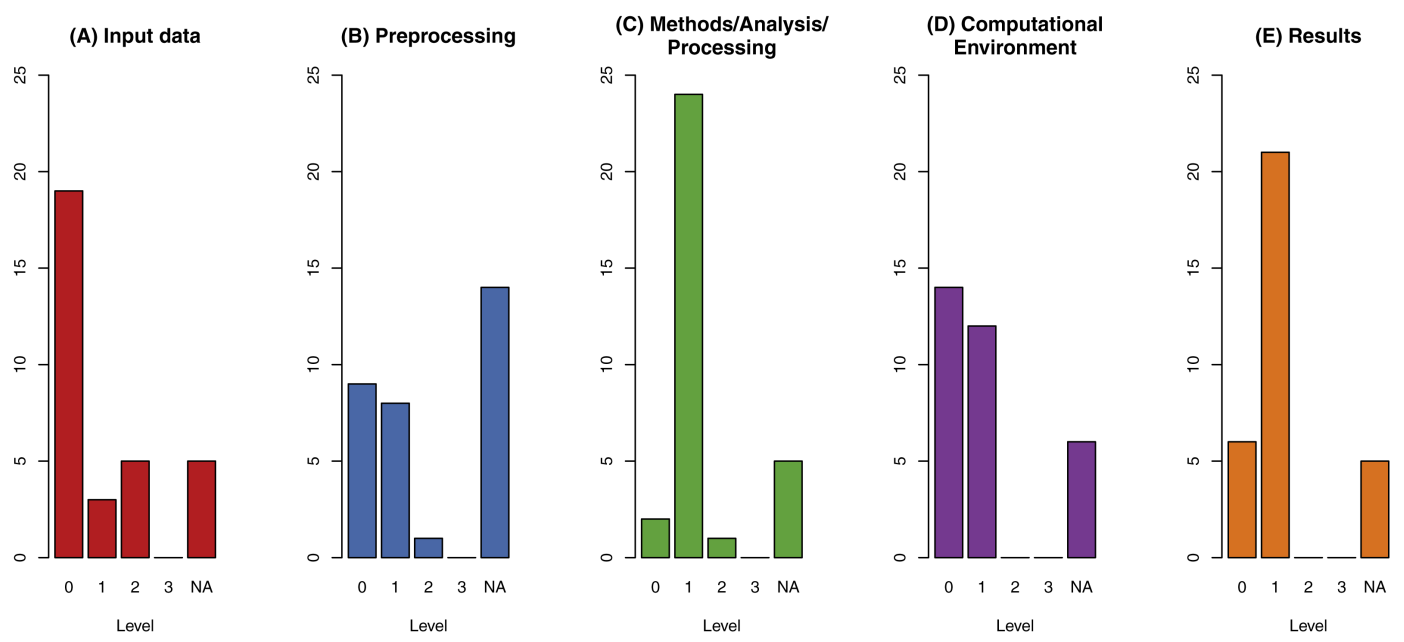

Fig. 2.3: Results of reproducibility assessment across all categories for the assessment of reproducibility: Data (A), Methods with sub-categories preprocessing (B), method/analysis/processing (C), computational environment (D), and Results (E). The level of reproducibility ranges from 0 (not reproducible) to 3 (fully reproducible); For 5 conceptual papers, all categories are NA.

level across all categories. Especially problematic is the high number of papers (19) with level 0 for data, meaning that the specific data is not only unavailable, but it is not re-creatable from the information in the paper. Data preprocessing applies to 18 publications, and the levels are low. Only one publication has level 2. Concerning the methods and results criteria, 19 out of 32 papers have level 1 in both, meaning the text includes understandable documentation.

Figure 2.4 shows that average reproducibility levels are low and do not change significantly over time, with the mean over all categories being below level 1 for all years. The categories are ordinal variables, meaning they have an implicit order but an unknown "distance" between them. They can be compared ( 3 is higher than 2), but absolute differences in numbers must not be interpreted. Moving one level up from 0 to 1 is not the same as from 2 to 3 . Averaging on ordinal variables must be conducted with care: Mode and median are mostly seen as acceptable averaging functions for ordinal data, while the mean is seen inapplicable by some.

We decided not to use median or mode because they hide all differences between the categories. The mean should not be applied for a single paper, whereby all categories in a single paper are averaged, because different evaluation rules would be combined into a meaningless number. Being aware of these limitations and the small dataset size, we opted to apply the mean and a statistical summary to categories to compare values between the different categories, and to compare the two large groups within the paper corpus (full and short papers). 


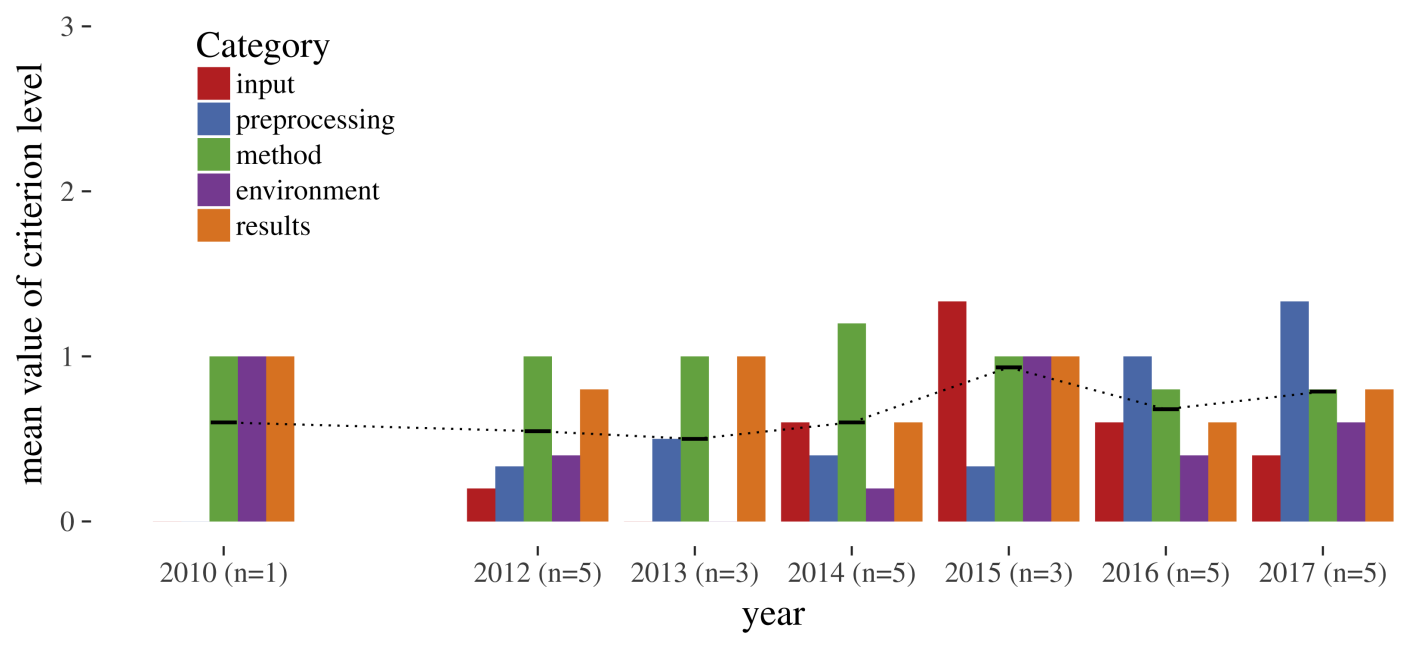

Fig. 2.4: Mean reproducibility levels per category over time; black dotted line connects the mean per year over all categories (in 2010 only one of three papers could be assessed, reaching level 1 for methods).

Tab. 2.4: Statistics of reproducibility levels per criterion.

\begin{tabular}{|l|c|c|c|c|c|}
\hline & Input data & Preproc. & Method/analysis/proc. & Comp. env. & Results \\
\hline Min. & 0.00 & 0.00 & 0.00 & 0.00 & 0.00 \\
\hline Median & 0.00 & 0.50 & 1.00 & 0.00 & 1.00 \\
\hline Mean & 0.48 & 0.56 & 0.96 & 0.46 & 0.78 \\
\hline Max. & 2.00 & 2.0 & 2.00 & 1.00 & 1.00 \\
\hline NA's & 5.00 & 14.0 & 5.00 & 6.00 & 5.00 \\
\hline
\end{tabular}

Tables 2.4 and 2.5 contain summary statistics per criterion and means for full and short papers. For each criterion, full papers reach higher levels of reproducibility than short papers (see Table 2.5).

\subsubsection{Author feedback (survey)}

This paper's repository (Nüst, 2018) includes the full survey responses. The survey was sent to authors via e-mail and was open from 23 October to 24 November 2017. In the case of obsolete e-mail addresses, we searched for updated ones and resent the form. Out of a total of 82 authors, 22 filled in the survey, resulting in responses for 17 papers, because six participants did not give consent to use their answers, two authors participated twice for different papers, and some papers had more than one individual response.

Authors were asked to comment on whether they agreed or disagreed with our evaluations of their specific paper. Seven responses fully agreed with the evaluation, five agreed partly, two expressed disagreement, and one did not answer the question. Most disagreements addressed the definition of criteria. Multiple authors argued 
Tab. 2.5: Mean levels per criterion for full and short papers.

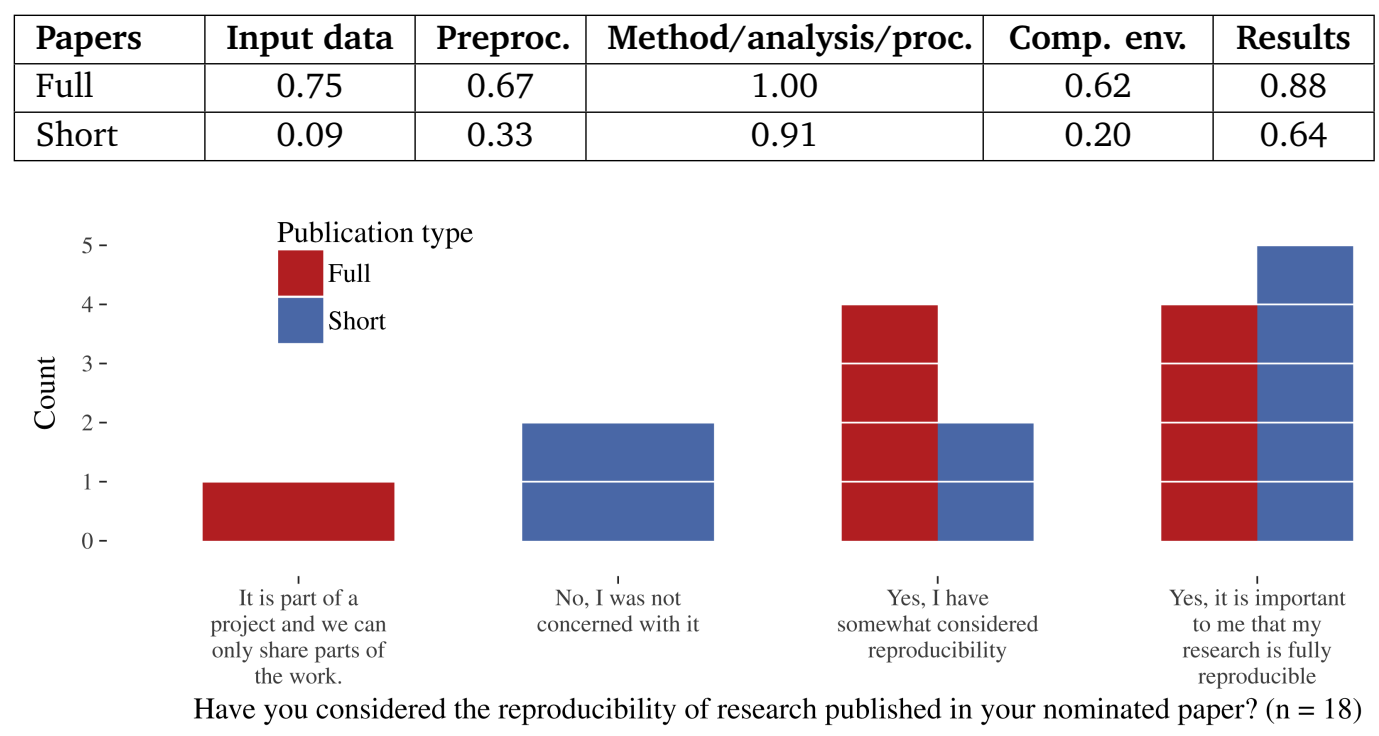

Fig. 2.5: Author survey results on the importance of reproducibility.

that such requirements should not be applicable for short papers, and that specific data is not always necessary for reproducibility. Others disagreed about treating "availability upon request" as "unavailable". One argued that OpenStreetMap data is by default "open and permanent", but for our criteria citing OpenStreetMap lacked direct links to specific versioned subsets of data.

The answers suggest that authors are generally aware of the need for reproducibility and in principle know how to improve it in their work. However, many do not consider it a priority, saying that they did not incorporate reproducibility because of a lack of motivation (eight respondents) or the extra effort required, which they say is disproportionately large in comparison to the added value.

According to the survey results, reproducibility was important to more than half of the respondents (see Figure 2.5). Only two respondents claimed they were not at all concerned about it (both short papers). Further comments revealed that some authors consider short papers as introductions of new concepts and generally too short for reproducibility concerns. The paper corpus supports this opinion because short papers reach overall lower reproducibility levels.

In contrast, we argue that transparency should not depend on the publication type but is a feature of the entire scientific process. Especially in the early stages, the potential for external review and collaboration can be beneficial for authors. Further, putting supplementary materials in online repositories addresses the problem of word count limits (for full and short papers), with which many authors struggle. 
To identify barriers to reproducibility, the authors were asked to rate how strongly five predefined barriers (Table 2.2) impacted their work's reproducibility. They could also add their reasons, for which they mentioned paper length restrictions and the need for additional financial resources. Table 2.6 shows that the most frequently mentioned reasons were legal restrictions and lack of time, where only one respondent indicated that these factors played no role. Although a lack of knowledge on how to include data, methods, and results was not considered by many as a barrier, several respondents noted a lack of supporting tools as a primary impediment for reproducibility.

Respondents also shared their ideas for how AGILE could encourage reproducibility in its publications. Four suggested open access publishing and asked for solutions to deal with sensitive data. A few suggested encouraging and promoting collaboration across research institutes and countries to mitigate temporary storage and organizations. Some respondents proposed that an award could be given for reproducible papers, reproducibility could be a requirement for the best paper nomination, or conference fees could be waived for reproducible papers. In summary, almost all authors agreed on the importance of the topic and its relevance for AGILE.

\subsection{Discussion}

\subsubsection{A critical review of this paper's reproducibility}

We acknowledge that this paper has its shortcomings concerning reproducibility. The data, code, and a description of the runtime environment are accessible on GitHub ${ }^{17}$ and deposited in an open repository on Zenodo ${ }^{18}$ (Nüst, 2018). The repository comprises an anonymized table with the survey results and a literate programming document, which transparently combines data preprocessing, analysis, and visualizations. The runtime environment description is based on Docker ${ }^{19}$ and allows readers to quickly open an interactive analysis environment in their browser based on Binder $^{20}$ (cf. Holdgraf, 2017). The working link to launch the binder is https: //mybinder.org/v2/gh/nuest/reproducible-research-and-giscience/6 (last access: 01.04.2019) and the file README.md provides instructions on the usage. The input data (i.e. the paper corpus) for the text analysis cannot be re-published due to copyright restrictions. Our sample is biased (although probably positively), as we only considered award nominees. Access to all papers would have allowed a random sample from the population. Regarding the method, the created criteria

\footnotetext{
${ }^{17}$ GitHub project: https://github.com/nuest/reproducible-research-and-giscience, last access for this and the following URLs: 01.04.2019.

${ }^{18}$ Zenodo: https://doi.org/10.5281/zenodo.1227260

${ }^{19}$ Wikipedia article about Docker: https://en.wikipedia.org/wiki/Docker_(software)

${ }^{20}$ Binder: http: //mybinder .org/
} 
Tab. 2.6: Hindering circumstances for reproducibility for each survey response $(\mathrm{n}=17)$ sorted by barrier type for the category with most "Main reason" occurrences; each line is one response.

\begin{tabular}{|c|c|c|c|c|}
\hline $\begin{array}{c}\text { Legal } \\
\text { restrictions }\end{array}$ & Lack of time & Lack of tools & $\begin{array}{c}\text { Lack of } \\
\text { knowledge }\end{array}$ & $\begin{array}{c}\text { Lack of } \\
\text { incentives }\end{array}$ \\
\hline Main Reason & $\begin{array}{l}\text { Strongly } \\
\text { hindered }\end{array}$ & Not at all & Not at all & $\begin{array}{l}\text { Strongly } \\
\text { hindered }\end{array}$ \\
\hline Main Reason & Not at all & Not at all & Not at all & $\begin{array}{l}\text { Moderately } \\
\text { hindered }\end{array}$ \\
\hline Main Reason & $\begin{array}{c}\text { Slightly } \\
\text { hindered }\end{array}$ & $\begin{array}{l}\text { Strongly } \\
\text { hindered }\end{array}$ & $\begin{array}{l}\text { Moderately } \\
\text { hindered }\end{array}$ & $\begin{array}{l}\text { Strongly } \\
\text { hindered }\end{array}$ \\
\hline Main Reason & Not at all & $\begin{array}{c}\text { Slightly } \\
\text { hindered }\end{array}$ & Not at all & Not at all \\
\hline $\begin{array}{l}\text { Strongly } \\
\text { hindered }\end{array}$ & $\begin{array}{l}\text { Strongly } \\
\text { hindered }\end{array}$ & $\begin{array}{l}\text { Strongly } \\
\text { hindered }\end{array}$ & $\begin{array}{c}\text { Moderately } \\
\text { hindered }\end{array}$ & $\begin{array}{l}\text { Strongly } \\
\text { hindered }\end{array}$ \\
\hline $\begin{array}{l}\text { Moderately } \\
\text { hindered }\end{array}$ & Main Reason & Not at all & Not at all & Not at all \\
\hline $\begin{array}{c}\text { Slightly } \\
\text { hindered }\end{array}$ & $\begin{array}{l}\text { Moderately } \\
\text { hindered }\end{array}$ & $\begin{array}{c}\text { Slightly } \\
\text { hindered }\end{array}$ & $\begin{array}{c}\text { Slightly } \\
\text { hindered }\end{array}$ & $\begin{array}{l}\text { Moderately } \\
\text { hindered }\end{array}$ \\
\hline $\begin{array}{l}\text { Slightly } \\
\text { hindered }\end{array}$ & Not at all & Main Reason & $\begin{array}{l}\text { Strongly } \\
\text { hindered }\end{array}$ & Not at all \\
\hline Not at all & $\begin{array}{l}\text { Moderately } \\
\text { hindered }\end{array}$ & Not at all & $\begin{array}{c}\text { Moderately } \\
\text { hindered }\end{array}$ & Not at all \\
\hline Not at all & $\begin{array}{l}\text { Strongly } \\
\text { hindered }\end{array}$ & $\begin{array}{l}\text { Strongly } \\
\text { hindered }\end{array}$ & $\begin{array}{l}\text { Strongly } \\
\text { hindered }\end{array}$ & $\begin{array}{c}\text { Slightly } \\
\text { hindered }\end{array}$ \\
\hline Not at all & $\begin{array}{l}\text { Moderately } \\
\text { hindered }\end{array}$ & Not at all & Not at all & Not at all \\
\hline Not at all & $\begin{array}{c}\text { Slightly } \\
\text { hindered }\end{array}$ & Main Reason & Not at all & $\begin{array}{l}\text { Strongly } \\
\text { hindered }\end{array}$ \\
\hline Not at all & Main Reason & Not at all & Not at all & Not at all \\
\hline Not at all & Main Reason & Not at all & Not at all & Not at all \\
\hline Not at all & $\begin{array}{l}\text { Moderately } \\
\text { hindered }\end{array}$ & $\begin{array}{l}\text { Moderately } \\
\text { hindered }\end{array}$ & Not at all & $\begin{array}{l}\text { Strongly } \\
\text { hindered }\end{array}$ \\
\hline Not at all & Not at all & Not at all & Not at all & Not at all \\
\hline Not at all & $\begin{array}{l}\text { Slightly } \\
\text { hindered }\end{array}$ & Not at all & $\begin{array}{c}\text { Slightly } \\
\text { hindered }\end{array}$ & Not at all \\
\hline
\end{tabular}


and how humans assigned them cannot honor all details and variety of individual research contributions and is inherently subjective. We tried to mitigate this by applying a "four eyes" principle, and transparently sharing internal comments and discussion on the matter in the code repository. Using our classification, we critically assign ourselves level 0 for data and level 3 for methods and results.

\subsubsection{Improving day-to-day research in GIScience}

Our evaluation identifies issues of reproducibility in GIScience. Many of the evaluated papers use data and computer-based analysis. All papers were nominated for the best paper award within a double-blind peer review and thus represent the upper end of the quality spectrum at an established conference. Overall reproducibility is low, and no positive trend is perceivable. It seems that current practices in scientific publications lack full access to data and code. Instead, methods and results are only documented as part of the text.

In order to significantly improve the reproducibility of research, there must be changes in educational curricula, lab processes, universities, journal publishing, and funding agencies (Yale Law School Roundtable on Data and Code Sharing, 2010; McKiernan, 2017) as well as reward mechanisms that go beyond paper citations (cf. term "altmetrics" in Priem et al. (2017)). This process is a major and longterm endeavor. Here, we focus on recommendations and suggestions for individual researchers and a specific organization: AGILE. A snowball effect may lead to a change in practices in the GIScience community. The remainder of this paper addresses RQ 4 by formulating suggestions to researchers and the AGILE conference organizers.

\subsubsection{Suggestions to authors}

Regarding habits and workflows, the Carpentries (the union ${ }^{21}$ of Data Carpentry (Teal et al., 2015) and Software Carpentry (Wilson, 2006) offer lessons on tools to support research, such as programming and data management, across disciplines. Further resources are available from programming language and software communities, research domains, and online universities. Often these resources are available for free because the software is Free and Open Source Software (FOSS) and driven by a mixed community of users and developers. Ultimately, proprietary software is a deal-breaker for reproducibility (cf. Ince et al., 2012; Baker, 2016b). OSGeoLive $^{22}$ provides a simple environment to test open alternatives from the geospatial

\footnotetext{
${ }^{21}$ Joint future for Software and Data Carpentry: http://www . datacarpentry.org/blog/merger/, last access for this and the following URL: 01.04.2019.

${ }^{22}$ OSGeo-Live: https : //live.osgeo.org/
} 
domain, and several websites offer help in finding FOSS comparable to commercial products $^{23}$. However, authors can do more than use open software to improve reproducibility. It is not only about the software. They can engage in simple tasks such as "naming things" sensibly ${ }^{24}$, they can be realistic by not striving for perfection but following "good enough practices in scientific computing" (Wilson et al., 2017), they can explore "selfish reasons to work reproducibly" (Markowetz, 2015), and they can follow FAIR ${ }^{25}$ guidelines with "structuring supplemental material" (Greenbaum et al., 2017).

\subsubsection{Recommendations to conferences in GIScience and organizations like AGILE}

What can conferences and scientific associations do to encourage reproducibility? A crucial step in improving reproducibility of GIScience research is acknowledging the important role organizations like AGILE can play in adopting reproducible research practices, which can build upon a large body of guidelines, documentation, and software. In the remainder of this section, we propose concrete actions for organizations, using AGILE as the leading example.

AGILE could show that it recognizes and supports reproducibility by offering an award for reproducible papers. This award is already provided by other communities, e.g. the ACM SIGMOD 2017 Most Reproducible Paper Award ${ }^{26}$. At AGILE, when reviewers suggest submissions for best (short) papers, they could also have these papers briefly checked for reproducibility. This check could be the task of a new Scientific Reproducibility Committee led by a Reproducibility Chair, working alongside the existing committees and their chairs. Committee membership would be publicly recognized. The "most reproducible paper" could be prominently presented in the conference's closing session.

Kidwell et al. (2016) demonstrated that open data badges had a positive effect on actual publishing of data in the journal Psychological Science, which uses badges and corresponding criteria from the "Center for Open Science"27 (COS). Further examples are the "kite marks" used by the journal Biostatistics (Peng, 2011), the common standards and terms for artifacts used by the Association for Computing

\footnotetext{
${ }^{23}$ see e.g. https://opensource.com/alternatives or https://alternativeto. net, last access for this and the following URLs: 01.04.2019.

24 Speakerdeck: https://speakerdeck.com/jennybc/how-to-name-files by Jennifer Bryan

${ }^{25}$ Force11.org. Guiding principles for findable, accessible, interoperable and reusable data publishing: version B1.0. https://www. force11.org/node/6062

${ }^{26}$ ACM SIGMOD 2017 Most Reproducible Paper Award: http://db-reproducibility.seas. harvard.edu/ and https://sigmod.org/2017-reproducibility-award/

${ }^{27}$ Center for Open Science: https://osf .io/tvyxz/wiki/home/
} 
Machinery's (ACM) ${ }^{28}$, and the Graphics Replicability Stamp Initiative (GRSI) ${ }^{29}$. While AGILE could invent own badges, reusing existing approaches has practical advantages (no need to design new badges), organizational advantages (no need to reinvent criteria), and marketing advantages (higher memorability). Author guidelines would include instructions on how to receive badges for a submission. The evaluation of badge criteria would be integrated into the review and could inform the reproducible paper award.

Author guidelines are the essential place to set the foundation for a reproducible conference (cf. SIGMOD $2018 \mathrm{CFP}^{30}$ ). Independently of advertising awards and badges, author guidelines should include clear guidelines on when, how, and where to publish supplemental material (data, code). Author guidelines for computational research must make authors aware of highlighting reproducibility-related information for reviewers and readers. These guidelines should contain practical advice, such as code and data licenses ${ }^{31}$, and instructions on how to work reproducibly, such as by providing a space for sharing tools and data, which is the most popular suggestion from the survey (seven respondents).

While the established peer-review process works well for conceptual papers, a special track or submission type ${ }^{32}$ could accommodate submissions focusing on reproducibility without an original scientific contribution and an adapted process (e.g. public peer review). Such publications can include different authors, e.g. technical staff, or even reviewers as practiced by Elsevier's Information Systems journal. Publications in an exclusive track can also mitigate limitations on research paper lengths. Unfortunately, they can also convey the counterproductive message of reproducibility being cumbersome and uncommon.

Submissions through this special track as well as the regular conference proceedings should be published as open access ${ }^{33}$ content in the future. It might even be possible to re-publish short papers and abstracts of previous conferences after solving juridical concerns (e.g. if author consent is required). To do this, AGILE could utilize existing repositories or operate its own, where using third party repositories ${ }^{34}$

${ }^{28}$ ACM: https://www.acm.org/publications/policies/artifact-review-badging, last access for this and the following URLs: 01.04.2019.

${ }^{29}$ GRSI: http://www .replicabilitystamp.org/

${ }^{30}$ SIGMOD 2018 CFP: https://sigmod2018.org/calls_papers_sigmod_research.shtml

${ }^{31}$ e.g. OSI compliant for code and Open Definition compliant for data, see http://licenses. opendefinition.org/.

${ }^{32}$ See IEEE's CiSE magazine's Reproducible Research Track https://www.computer.org/cise/ 2017/07/26/reproducible-research-track-call-for-papers/, and Elsevier journal Information Systems' section for invited reproducibility papers https ://www . elsevier. com/journals/ information-systems/0306-4379/guide-for-authors.

${ }^{33}$ Open access: https://open-access.net/DE-EN/information-on-open-access/ open-access-strategies/

${ }^{34}$ Beside the incumbents Figshare (https://figshare.com/), Open Science Framework (OSF) (https://osf.io/, community-driven) and Zenodo (https://zenodo.org/, potentially prefer- 
for supplements would reduce the burden on the AGILE organization. Choosing one repository allows for collecting all AGILE submissions under one $\operatorname{tag}^{35}$ or community $^{36}$. An AGILE-specific repository would allow more control but would require more work and might have lower visibility, since search engines index the large repositories properly. Both approaches would support a double-blind review by providing anonymous view-only copies of supplemental material ${ }^{37}$.

We see AGILE, carried by its member labs and mission ${ }^{38}$, as being in a unique position among GIScience conferences to establish a common understanding and practice of reproducible research. Firstly, member labs can influence education, especially at the graduate level, and ideally, collaborate on open educational material. Completing a Ph.D. in an AGILE member lab and participating in AGILE conferences should qualify early career scientists to publish and review reproducible scholarly works. Secondly, the conference can take a leading role in setting up new norms for conference review and publication but at the same time cooperate with other conferences (e.g. ACM SIGMOD). At first AGILE would encourage but eventually demand the highest level of reproducibility for all submissions. This process certainly will take several years to complete.

\subsection{Conclusions}

What skills related to reproducibility are desirable for authors at GIScience conferences in 2028? Predicting ten years ahead might not be scientific, but it allows for formulating a vision to conclude this work. We assume that in 10 years, hardly any paper will not utilize digital methods, such as software for analysis, interactive visualizations, or open data. Ever more academics will face competitive selection processes, where its transparency and novelty will measure the quality of research. To achieve novelty in a setting where all research is saved, findable and potentially interpreted by artificial intelligence (Jones, 2016), a new contribution must be traceable. Thus, the trend towards "open science" will be reinforced until it is standard practice to use and publish open source code and open data as well as to incorporate alternative metrics beyond citations. As of now, AGILE is not ready for such research. It has identifiers (DOIs) only for full publications and lacks open

able given AGILE's origin because EU funds it), a large number of open access repositories exists, see http://roar.eprints.org/ and http://opendoar.org/, including platforms by publishers, e.g. Springer (https://www.springer.com/gp/open-access), or independent organizations, e.g. LIPIcs proceedings (https://www.dagstuhl.de/en/publications/lipics), last access for these and the following URLs: 01.04.2019.

${ }^{35}$ Tags: http://help.osf.io/m/sharing/1/524053-tags

${ }^{36}$ Community: https://zenodo.org/communities/

${ }^{37}$ Creating anonymized supplemental material: http://help.osf.io/m/links_forks/l/ 524049-create-a-view-only-link-for-a-project

${ }^{38}$ About AGILE: https://agile-online.org/agile-community 
licenses for posters and (short) papers. Statements on preprints (publication before submission) and postprints ("green" open access ${ }^{39}$ ) are missing.

Researchers, conference organizers, and program committees will have to leave their comfort zone and change the way they work. Also, in order to overcome old habits, they will have to immediately see the benefits of the new ways (Wilson et al., 2017). The evidence for the benefits of "open science" is strong (McKiernan et al., 2016), but in order to succeed, the community must embrace the idea of a reproducible conference. We acknowledge that fully reproducible GIScience papers are no small step for both authors and reviewers, but making them the standard would certainly be a giant leap for GIScience conferences. We are convinced that a conference like AGILE can provide the required critical mass and openness, and we hope the experiences and information provided in this work represent a sound starting point.

\subsection{Acknowledgements}

We thank all authors who participated in the survey and the reviewers for their detailed comments and valuable suggestions for the manuscript. We want to thank Celeste R. Brennecka, from the Science Writing Support Service of the University of Münster, for her editorial support.

\footnotetext{
${ }^{39}$ Open access strategies: https://open-access.net/DE-EN/information-on-open-access/ open-access-strategies/, last access: 01.04.2019.
} 



\title{
Computational reproducibility in
} geoscientific papers: Insights from a series of studies with geoscientists and a reproduction study

\author{
This chapter was published as: Konkol, Markus, Christian Kray, and Max Pfeiffer \\ (2018). "Computational reproducibility in geoscientific papers: Insights from a \\ series of studies with geoscientists and a reproduction study". In: International \\ Journal of Geographical Information Science, 1-22. eprint: https://doi.org/10. \\ $1080 / 13658816.2018 .1508687$. It corresponds to P3 in the list of publications.
}

\begin{abstract}
Reproducibility is a cornerstone of science and thus for geographic research as well. However, studies in other disciplines such as biology have shown that published work is rarely reproducible. To assess the state of reproducibility, specifically computational reproducibility (i.e. re-running the analysis of a paper using the original code), in geographic research, we asked geoscientists about this topic using three methods: a survey $(n=146)$, interviews $(n=9)$, and a focus group $(n=5)$. We asked participants about their understanding of open reproducible research $(O R R)$, how much it is practiced, and what obstacles hinder ORR. We found that participants had different understandings of $O R R$ and that there are several obstacles for authors and readers (e.g. effort, lack of openness). Then, in order to complement the subjective feedback from the participants, we tried to reproduce the results of papers that use spatial statistics to address problems in the geosciences. We selected 41 open access papers from Copernicus and Journal of Statistical Software and executed the $R$ code. In doing so, we identified several technical issues and specific issues with the reproduced figures depicting the results. Based on these findings, we propose guidelines for authors to overcome the issues around reproducibility in the computational geosciences.
\end{abstract}




\subsection{Introduction}

Reproducibility is an essential element of scientific work in general, as it enables researchers to re-run and reuse experiments reported by others. Further benefits of working and publishing reproducibly include increased transparency and more efficient review processes (Gil et al., 2016). Despite these advantages, publishing results in a reproducible way is still not common practice (Reichman et al., 2011), which is part of the reason why some have proclaimed a "reproducibility crisis" (Baker, 2016a). A recent study in economics (Gertler et al., 2018) has shown that even when authors make the data and code publicly accessible, it is not guaranteed that readers can successfully reproduce the results published in the paper. On top of that, the inconsistent usage of the terms reproducibility and replicability within and across disciplines can cause further confusion (Bollen et al., 2015). It is thus not surprising that the topic of reproducible research and how to realize it are discussed across many disciplines such as biology (Leek and Jager, 2017) and computer science (Stodden, 2010).

In this article, we focus on computational reproducibility in general and open reproducible research (ORR) in particular. Goodman et al. (2016) state that in ORR all used research components, e.g. data, software, and configuration are publicly accessible and produce the same results (i.e. numbers, tables, figures) compared to those reported in the paper. This form of publishing research is particularly relevant in the geosciences which encompass all domains related to earth sciences, such as climatology and landscape ecology (see the list of geoscientific domains by Nature Geosciences (2018)). According to Goodchild (1992), three relevant topics in geographic information science are spatial statistics, algorithms that operate on geographic information, and the display of geographic information. Many papers published in the geosciences apply spatial statistics based on geographic information, and the results are often displayed as maps or time series. Thus, to achieve a minimum standard of credible research results, computational reproducibility is essential. However, compared to other disciplines, the field of computational geosciences (geoscientific research based upon code and data) has given little attention to reproducibility (cf. Giraud and Lambert, 2017). This paper aims to address this gap by investigating how geoscientists who conduct computational research understand reproducibility, whether and how it is practiced, and what obstacles hinder it. Hence, we carried out three studies with geoscientists (a survey, interviews, and a focus group), and we performed a reproduction study using previously published reports that apply spatial statistics in $R$.

Contributions. This article contributes to the following insights. First, we report on what geoscientists understand $O R R$ to mean. Second, we identify practical obstacles 
that stand in the way of authors publishing $O R R$, and we also identify some obstacles that readers face when reproducing others' work. Third, from our reproducibility study, we report on technical issues when attempting to execute the original code provided in the papers we aimed to reproduce. Next, we describe key differences that impeded the comparability of the original and our reproduced figures. Finally, we propose a set of guidelines for authors to address the identified issues.

Scope. Reproducibility is a complex concept involving different stakeholders (see chapter 4) across multiple disciplines. To keep the scope of the research manageable, we do not consider qualitative research and how to reproduce it, and amongst all stakeholders, we only focus on authors and readers.

In the following, we first review related work on reproducible research in general and in particular in the geosciences. Then, we report on the four studies we conducted here, i.e. the survey, the interviews, the focus group, and the reproducibility study. We then discuss our findings and their limitations. We conclude by summarizing key insights and providing a set of guidelines for authors wishing to publish reproducibly.

\subsection{Related Work}

We first review work on the various definitions of reproducible research, the obstacles authors and readers face when producing and using reproducible research, the incentives for publishing reproducibly, and approaches to overcome the associated barriers.

\subsubsection{Reproducible research}

Different definitions of and perspectives on reproducible research have been proposed. According to Leek and Peng (2015), research results reported in a paper are reproducible if they can be re-computed based on the same data and "knowledge of the data analysis pipeline". Easterbrook (2014) considers research to be reproducible if it enables the re-creation of the results based on given code or an own program. Both definitions are flexible regarding the use of procedures and software. In contrast, Gentleman and Lang (2007) require that for authors' research to be called reproducible, they must include the software they used to produce their results. Similarly, Bollen et al. (2015) equates reproducibility with being able to achieve the same results as reported using the same data and procedure. Goodman et al. (2016) linked reproducibility to specific purposes: they distinguish between methods reproducibility, referring to achieving the same results based on the same data and 
code, and results reproducibility, which corresponds to replicability, i.e. achieving consistent results by independent experiments with new data and code. Peng (2011) proposed a reproducibility spectrum ranging from not reproducible (if no research materials are provided), to reproducible (if code and data are available), to fully replicable. In contrast, Leek and Jager (2017) make a binary distinction based on the outcome of an attempt to reproduce results: research is reproducible if the results are the same; if they are not, the research is not reproducible. In summary, we can thus note that while the basic notion of reuse is consistent across different definitions of reproducibility, there are also substantial differences: some definitions simply require a detailed methodology section whereas others demand access to all used materials (e.g. data and software).

\subsubsection{Incentives for publishing reproducible research}

Independent of what definition of reproducibility is used; there are a number of reasons why it makes sense for authors to publish reproducible work and for readers to make use of such work. Most importantly, reproducible research facilitates the reuse of the results in the paper, including the methods and data that were used to produce the results (Collberg and Proebsting, 2016; Gil et al., 2016). Furthermore, detecting errors is easier if research is reproducible (Gentleman and Lang, 2007), such as when there are differences between the reported and replicated results (Donoho et al., 2009). Readers or reviewers can then check if there was an error in the original analysis, e.g. by studying the data analysis and parameters that were used (Stodden et al., 2016). Moreover, it is likely that reproducibility will become a requirement for reputable publication outlets (Gil et al., 2016); working reproducibly from the beginning can make it easier to meet a journal's standards (Hillebrand and Gurevitch, 2013). With the growing trend towards "open science" including open data, open code (Gewin, 2016), transparency can be increased, and the credibility crisis can be tackled (Reichman et al., 2011).

Further benefits of reproducible research arise from new possibilities afforded by the approach, e.g. meta-analyses (Stodden et al., 2016), continuously evolving papers (Brunsdon, 2016), and new cooperations (Costello, 2009). Journals such as Distill ${ }^{1}$ support publication of transparent research that can include, e.g. interactive figures. The ReScience $e^{2}$ journal encourages replication of the computational steps in published articles, ideally as open source implementations for future use. Finally, providing public access to code and data increases citation numbers (Piwowar et al., 2007; Vandewalle, 2012), which have a direct impact on researchers' reputations. Despite these benefits, it is essential to keep in mind that reproducible research cannot

\footnotetext{
${ }^{1}$ Distill: https://distill.pub/journal/, last access for this and the following URL: 01.04.2019.

${ }^{2}$ ReScience: http://rescience.github.io/
} 
prevent flaws during data collection (Ostermann and Granell, 2017). Nevertheless, it can help to establish a minimum standard for credible computational research (Bollen et al., 2015).

\subsubsection{Reasons for unreproducible papers}

Given the long list of benefits and incentives for publishing reproducibly, it might seem surprising that not all research is published in this way. There are, however, a number of different reasons that explain why most papers are published in a non-reproducible way. These reasons include cultural (Reichman et al., 2011) and technical (Easterbrook, 2014) barriers as well as authors who cannot reproduce their own results (Vandewalle et al., 2009). One key issue is that data is rarely available (Ioannidis et al., 2009). If not archived, data availability declines with article age, making it particularly challenging to reproduce older publications (Vines et al., 2014). A second issue is the source code is rarely accessible, and if it is, it is not always in the right version (Collberg and Proebsting, 2016). This issue is caused mainly by the considerable effort required for preparing code and data for publication (Barnes, 2010). Furthermore, authors are frequently not aware of the incentives that might be worth the extra effort (Nosek et al., 2015) and of the drawbacks of not publishing reproducibly, e.g. having to respond to questions about the code (Gewin, 2016). Another problem is that many scientists worry about falling behind (Gewin, 2016) if they spend time "unwisely", as the credit system does not sufficiently reward scientists for fully disclosing their own work (McCullough et al., 2008). In addition, researchers fear that if they are fully transparent, others question their conclusions (Piwowar et al., 2007) and thereby tarnish their reputation; but this is, in fact, an essential process in science (Benestad et al., 2016). Further barriers to reproducible research are legal aspects, sensitive data (Stodden et al., 2016), and ethical concerns (Darch and Knox, 2017). As a result of these issues, some have proclaimed that science is suffering from a "reproducibility crisis" (Baker, 2016a). Examples that support this claim highlight the drawbacks of unreproducible papers including a study with 100 replication attempts by Open Science Collaboration (2015) and the flaws detected in published articles (cf. Benestad et al., 2016).

\subsubsection{Guidelines and recommendations}

Several authors have proposed ways to overcome the issues and barriers outlined in the previous subsection. Nosek et al. (2015) presented eight Transparency and Openness Promotion (TOP) guidelines addressing, for example, citation standards for materials, sharing of data and methods, and preregistration. Each guideline has four levels ranging from standard not met to standard fully met. Based on these guidelines, 
Stodden et al. (2016) suggested the Reproducibility Enhancement Principles (REP) for computational research, but they also highlighted that journals should demand all research components underlying the analysis. Ideally, these components should be shared via public repositories and archives (Gewin, 2016). Another recommendation is to design an improved credit system to address citation issues such as being able to cite individual research components (Gil et al., 2016). Consequently, all citable components will need a legal statement on reusability (Stodden et al., 2016). Scientists should choose open source software instead of proprietary tools, and they should add information on the computational environment (Fehr et al., 2016). Both practices facilitate reproduction by third parties, and Steiniger and Hay (2009) also showed that free tools can be as useful as proprietary software. Moreover, figures should be created by using scripts instead of ready-to-use toolboxes, which hide the computational steps (Sandve et al., 2013) and hinder reproduction. In this context, being able to reproduce figures is particularly important, as they are popular means to visualize computational results (cf. Claerbout and Karrenbach, 1992). Several technical solutions support scientists in publishing reproducible research. A popular approach is literate programming, which allows authors to combine text and code into a single document (Knuth, 1984), e.g. using RMarkdown (Allaire et al., 2018) or Jupyter Notebooks (Kluyver et al., 2016). This approach can be extended to reproducible books such as the openly developed book Geocomputation with $R$ by Lovelace et al. (2016), to which everyone can contribute. A useful tool for sharing code is $\mathrm{GitHub}^{3}$, a popular software development platform. However, one issue with publishing material on publicly accessible platforms is that it can interfere with a double-blind reviewing process. To counter this issue, the "Open Science Framework" ${ }^{4}$ also enables sharing materials anonymously for peer review.

\subsubsection{Reproducible research in the geosciences}

Ostermann and Granell (2017) investigated if research on volunteered geographic information (VGI) is reproducible, i.e. by using the same data and methods, and is replicable, i.e. by conducting an independent experiment with new data and a similar method. According to their results, none of the investigated publications were reproducible and less than half of them were replicable. In order to facilitate reproducibility, Gil et al. (2016) proposed the Geoscience Paper of the Future, which provides public access to research components enriched by metadata. Giraud and Lambert (2017) argued that figures, such as maps, frequently depict key results of geographic research and thus should be reproducible. Brunsdon (2016) investigated the importance of code in quantitative geography. Key observations were that making code available facilitates tasks, such as comparing different implementations of an

\footnotetext{
${ }^{3}$ GitHub: https://github.com/, last access for this and the following URL: 01.04.2019.

${ }^{4}$ Open Science Framework: https://osf .io/
} 
analysis. In addition, researchers can re-run the analysis with another or updated dataset. In this context, it is particularly important to provide the original code, as textual descriptions might be inaccurate. Increasingly, geoscientific journals such as Nature Geosciences encourage publishing code underlying the reported results. In the Vadose Zone Journal (Skaggs et al., 2015), authors can submit reproducible research articles by attaching data, code, and metadata. While this constitutes a big step towards making research fully reproducible, the problem of making the code executable on different machines and in different environments persists. In order to tackle this issue, chapter 4 proposes the Executable Research Compendium (ERC), which encapsulates the runtime environment and all research components underlying the analysis in a Docker container. This approach can thus lead to improved reusability, accessibility, and transparency.

In summary, we can observe that reproducible research has gained importance in computational geosciences in recent years. However, it is largely unknown what geoscientists who conduct computational research such as spatial statistics understand $O R R$ to mean, what roadblocks they face in realizing $O R R$, and what differences exist compared to other disciplines. In the next sections, we therefore report on a series of studies we conducted to shed light on these questions. While research on reproducibility mostly focused on the accessibility of materials, we took one further step and examined if the code attached to papers is actually executable. Then, we proceeded to compare the resulting figures to those in the original article.

\subsection{Methods}

In order to obtain an initial but comprehensive overview of reproducibility in the computational geosciences and to better understand obstacles impeding $O R R$, we ran four complementary studies: an online survey, semi-structured interviews, a focus group discussion with geoscientists who conduct computational research, and a reproducibility study. The combination of these methods enabled us to gather qualitative and quantitative data directly from researchers in the geosciences as well as to objectively assess whether recently published papers in the computational geosciences are reproducible. By interrelating both types of data, we hoped to be able to gain more profound insights than either method could offer on its own. To keep the scope of the research manageable, we focused on authors and readers as participants and a subset of publication outlets with readily available material. 


\subsubsection{Approaches}

Online survey: Online surveys are efficient means for collecting responses from a large number of people (Lazar et al., 2017). Our goal was to examine key aspects in reproducible research, i.e. the accessibility of code and data, published by scientists with a geoscientific background. We analyzed the data using descriptive statistics and diverging stacked bar charts as suggested by Heiberger and Robbins (2014).

Semi-structured interviews: To receive more profound insights into what geoscientists understand by the term $O R R$ and what obstacles they face when publishing $O R R$ or when reproducing others' work, we conducted semi-structured interviews. During interviews, participants can express their thoughts freely, and the interviewer can ask more concrete or follow-up questions if required (Lazar et al., 2017). We applied the grounded theory approach for data analysis (Glaser and Strauss, 1967). Focus group: In focus group discussions, participants can interact with each other, because such conversations may elicit opinions and ideas different from those mentioned in interviews (Glaser and Strauss, 1967). We thus organized a focus group session to complement the interviews and the other data we gathered. We used the same topics and the same grounded theory approach as we did with the interviews. Reproducibility study: In order to objectively assess the technical issues that make it difficult to reproduce others' work, we also conducted a reproducibility study. We systematically collected papers that had included source code written in $R$, and we then executed the analysis. During the study, we took note of any issues and how we were able to solve them. The resulting insights enabled us to derive recommendations for authors on how to avoid these issues. In the following sections, we report on each study and its key results in detail.

\subsubsection{Online survey}

We conducted an online survey in order to assess whether geoscientists who conduct computational research publish $O R R$.

\section{Participants}

We recruited respondents during a poster presentation at the European Geosciences Union General Assembly (EGU) 2016 ${ }^{5}$. In total, 13,650 scientists from 109 countries and several research areas $^{6}$ (e.g. biogeosciences, climatology) participated in the conference. In addition, we emailed 1,554 researchers who contributed to the conference with a poster or talk. To be included in the analysis, participants had to

\footnotetext{
${ }^{5}$ EGU: https://www. egu2016. eu/, last access for this and the following URL: 01.04.2019.

${ }^{6}$ See https://meetingorganizer.copernicus.org/EGU2016/sessions-of-special-interest/ ECS for a full list of research domains.
} 
submit the survey actively. In total, 215 geoscientists started filling out the survey, of which 146 completed it (mean $\mu=17$ years in research, standard deviation $\sigma=9$ years). In our analysis, we only included the responses by participants who had submitted the survey and who had a research background in the geosciences.

\section{Materials}

After defining $O R R$, the survey collected background information about the respondents, i.e. whether they were authors, readers, or both, and their research field. We asked them how often (i) they published re-computable results, how frequently their papers linked to (ii) the data used and (iii) the code employed, and (iv) how often they tried to reproduce the results of other researchers. If they answered (ii) and (iii) in any way other than "never", we also asked how frequently they included persistent identifiers (e.g. Digital Object Identifier (DOI)). Respondents answered the frequency questions using a five-item scale from "never" to "always". We evaluated the data with the help of descriptive statistics. The survey was available for six months (April-September 2016). All the materials we used for the survey, i.e. the questions, data, and code (reproducible RMarkdown document) are included in the supplements.

\section{Results}

Of those who responded to the survey, 49\% indicated they published their research often or always in a way that enables re-computation (Figure 3.1). However, only $33 \%$ included links to the data underlying the paper. Among those who included such links, $27 \%$ included persistent identifiers. Only $12 \%$ of all authors linked to the code used to produce the results, and among those who did, $12 \%$ included persistent identifiers. Of all survey respondents, $7 \%$ tried to reproduce other researchers' results often or always, and among those who answered this question other than never, $24 \%$ succeeded often or always. We can thus observe that there is a mismatch between those who said they published re-computable research and the frequency with which data and, particularly, code were shared. Figure 3.1 summarizes the responses obtained from all participants.

\subsubsection{Semi-structured interviews}

We conducted semi-structured interviews with geoscientists who conducted computational research to investigate their understanding of $O R R$ and to identify barriers to the realization of $O R R$. We recruited nine geoscientists (mean $\mu=9$ years in research) from geoinformatics, landscape ecology, geochemistry, and planetology (from within 


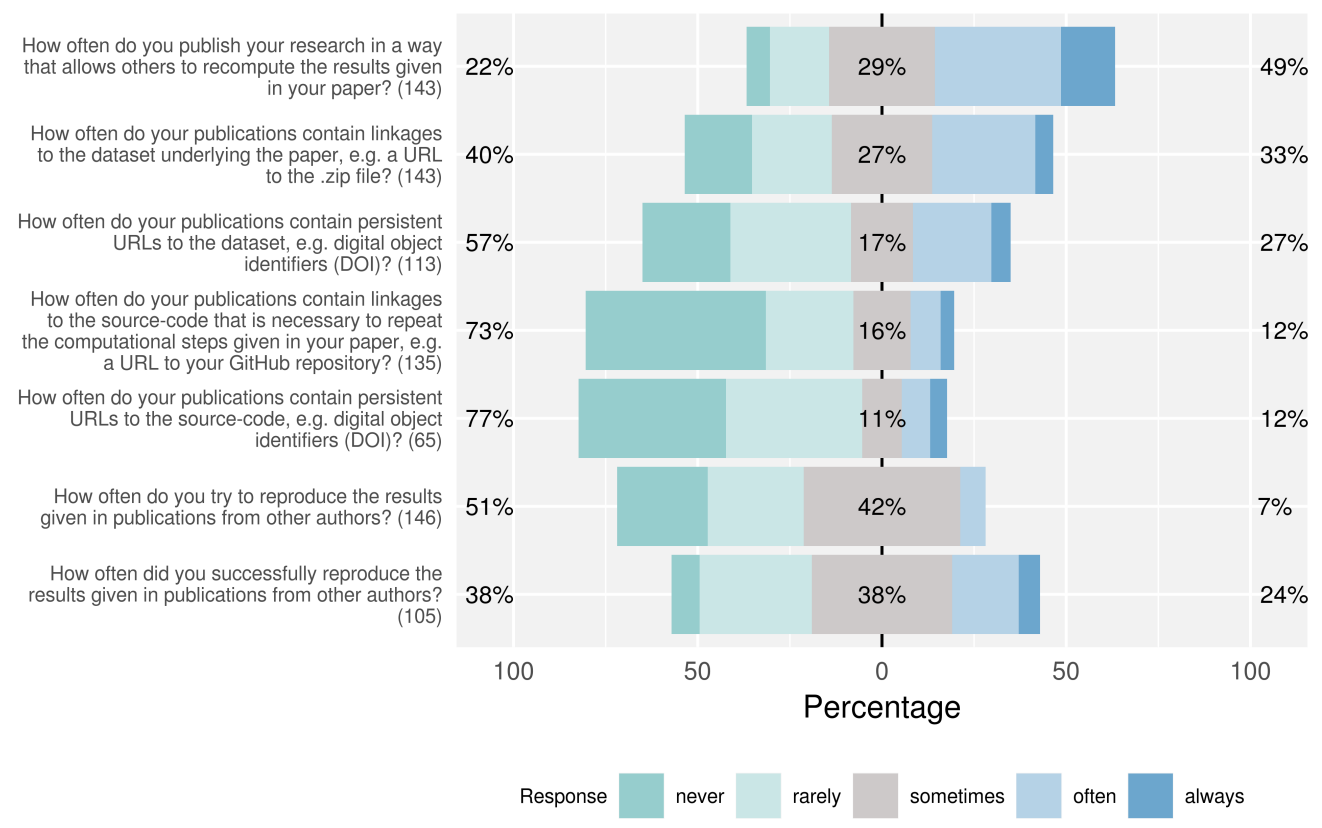

Fig. 3.1: The diverging stacked bar chart (Heiberger and Robbins, 2014) shows the percentage of respondents publishing reproducible research and reproducing the work of other researchers. Numbers in brackets show absolute numbers of respondents. Percentages are grouped into the categories never/rarely, sometimes, and often/always. Note: All except the last question were only shown to authors and not to readers.

our faculty ${ }^{7}$ ) who had previously published papers that included geospatial figures based on computations, e.g. maps or time series.

\section{Materials}

The interview began with a brief introduction to the overall topic. We then asked participants to explain how they understand ORR in three consecutive steps. First, we asked what is meant by reproducible research, then open research, and finally ORR. Next, we presented our definition (see above) so that we could continue the interview with a common understanding. We then asked for obstacles participants perceive that hinder them from publishing ORR (author's perspective) and prevent the reproduction of other researchers' work (reader's perspective). Finally, participants had to fill out a brief survey to collect background information about them (research field, years in research).

\footnotetext{
${ }^{7}$ Institute for Geoinformatics, University of Münster.
} 


\section{Procedure}

In order to ensure that the interview questions were understandable, we tested the interview with three Ph.D. students and revised the questions according to their feedback. All actual participants of the study received the final questions one day in advance. We recorded the interviews for later transcription (audio only). Before the interview started, participants were presented with a consent form that informed them about the audio recording, their rights, and their statements being treated anonymously. After they had signed it, we asked a series of questions during the actual interview and handed out a short questionnaire at the end. On average, the interview took 54 minutes (between 35 to $66 \mathrm{~min}$.). Seven interviews were conducted in German, two in English. We applied grounded theory (Glaser and Strauss, 1967) to analyze the data. We captured key statements and assigned these statements to codes ("open coding"), which were then grouped into higher level themes and finally into categories. The supplemental files to this article include all materials we used for the interview, i.e. the questions we used, the questionnaire, the statements from the interviewees, the codes and categories we derived as well as the consent form.

\section{Results}

In line with existing literature, we found that geoscientists have a divergent understanding of ORR. For eight interviewees, reproducible research should describe the methods that were used to produce the results in sufficient detail for them to be repeated by others. They expected that reproducing such studies would achieve consistent results (7 mentions) and that reproducible research should make materials, e.g. code and data, accessible (3). Accessible materials were also relevant in open research (5), which should also be transparent (3) and free of charge (3). Interviewees combined these aspects to describe $O R R$, i.e. public access to data (5), code, and methods (4) to achieve the same results (3). Moreover, in ORR there should be an explanation of how results were produced (3) and the research components should have non-restrictive licenses. Three associated the term with replicability, i.e. confirming results with independent experiments (Bollen et al., 2015).

Furthermore, interviewees named several obstacles hindering the publication of open and reproducible results and preventing the reproduction of other researchers' results (Table 3.1): Insufficiently described methods were seen as impeding the understanding of how results were produced. Frequently, materials needed for reproducing results were inaccessible due to scientists' fears or concerns (e.g. regarding legal issues). Another problem was the use of proprietary tools, which can encapsulate essential processes such as how results are computed and thus can 
Tab. 3.1: Obstacles while publishing reproducible research (left) and while reproducing other researchers' work (right). Numbers in brackets show how many interviewees mentioned the obstacle. Aspects marked with (*) were also mentioned in the focus group discussion.

\begin{tabular}{|l|l|}
\hline $\begin{array}{l}\text { Obstacles while publishing reproducible } \\
\text { research (authors) }\end{array}$ & $\begin{array}{l}\text { Obstacles while reproducing others' } \\
\text { work (readers) }\end{array}$ \\
\hline Describe methodology sufficiently (5) & Missing details in methodology (7)* \\
\hline Losing competitive advantages (4)* & Inaccessible materials (3)* \\
\hline Prepare code and data (4) & Not yet relevant (3)* \\
\hline Not yet relevant (4) & Proprietary software (2) \\
\hline Proprietary software (4) & Time consuming (2) \\
\hline Missing supporting tools (3) & Lack of expertise (1) \\
\hline Licensing (3)* & $\begin{array}{l}\text { Individual interpretations lead to other } \\
\text { conclusions* }\end{array}$ \\
\hline Code not worth publishing (2) & \\
\hline Making it understandable for non-experts (1) & \\
\hline
\end{tabular}

decrease transparency. In addition, several interviewees considered reproducible research as not being relevant for them yet. They also argued that their code was developed for a specific use case and thus not worth publishing, as others would not be able to reuse it. Finally, working reproducibly was seen as being too time consuming and not sufficiently supported by tools.

\subsubsection{Focus Group}

In order to complement the insights from the interviews regarding researchers' understanding of $O R R$ and the obstacles hindering $O R R$, we also conducted a focus group discussion. We recruited five additional geoscientists (mean $\mu=5$ years in research) from our faculty with backgrounds in landscape ecology and geoinformatics based on the same criteria we used for recruiting interviewees.

\section{Procedure}

The focus group discussion comprised the same three parts as the interviews. Participants received the guiding questions one day in advance. On the day of the focus group session, we used the same questionnaire and consent form. We briefly introduced the topic, asked participants to introduce themselves, and then asked the questions. The focus group took 86 minutes in total and was conducted in German. Statements were analyzed using the same grounded theory approach we applied for the interviews. All materials used for the focus group, i.e. the statements, codes and categories, the questionnaire, as well as the consent form are available in the supplements. 


\section{Results}

Participants of the focus group described their understanding of ORR one after another and also referred to each other. They collaboratively achieved the following definition: ORR contains a detailed description of the methodology ( 3 mentions) and provides access to data (2), model, and code (3). Readers can achieve consistent results (5) by repeating the analysis. This form of publishing scientific insights then leads to research being more transparent (5), because readers better understand how results were achieved and which limitations they have.

The participants of the focus group discussion made many statements that were similar to those mentioned in the interviews (cf. Table 3.1 for a summary of repeated statements). In addition to those statements, there were also a number of points that did not come up during the interviews. One participant pointed out that reproduction might fail due to individual interpretations that could differ from one researcher to another. Using different versions of the same software could result in some required functionality not being included or lead to deviating results due to the functionality having changed from one version to another. Thus, reproducible research is not necessarily achieved even when the used materials are accessible. To better understand the relevance of this observation, in our reproducibility study (below) we decided to reproduce results from papers that made available all their original materials, namely the spatial statistics and code.

\subsubsection{Reproducing geoscientific results}

A key benefit of $O R R$ is that other scientists can reuse existing materials such as code. However, this is only practical if the code is executable and produces the same results as those reported in the paper. In order to further examine obstacles for readers while reproducing published work, we tried to execute the code attached to papers and compared the figures depicting results in the reproduced and original versions.

\section{Materials}

We selected a paper for our study if it met the following criteria: (i) it was licensed as open access; (ii) it provided links to code written in $R$ (R Core Team, 2018), a programming language that is used frequently in the geosciences (Giraud and Lambert, 2017), and linked to data that was used (if applicable); and (iii) it was published between January 2016 and August 2017. The latter criterion was used in order to capture current practices of researchers and to keep the scope of the work manageable. We began our search by scanning the journals published by 
Copernicus Publications ${ }^{8}$, which are all open access and many of which fall into one of the aforementioned geoscientific domains. In the first run, we searched for papers using the keyword $R$ Core Team, as this term is used frequently to cite $R$ as the programming language underlying the developed software. In order to find cases where the authors did not cite the programming language but rather linked to the externally hosted code, we searched a second time using the term GitHub, which is a popular platform for storing and sharing source code. This two-step search yielded 31 research articles from the geoscientific domain that met our three criteria. Because computational analyses used in articles are often based upon other software libraries, we broadened our analysis and also considered the ten most cited papers of the Journal of Statistical Software 9 (state June 2016) that describe frequently used $R$ packages based on code written in $R$. Although these papers are not specific to the geosciences, they describe important features for spatial statistics, e.g. handling spatial and temporal data.

\section{Procedure}

In order to execute the code included in the papers, we set up RStudio (RStudio Team, 2015) on an Ubuntu system (Version: 16.04) using rocker/geospatial (Version 3.4.2), a Docker image tailored to the geoscientific domain (Boettiger and Eddelbuettel, 2017). If we encountered issues while running the scripts that we were unable to resolve by ourselves, we searched the Web for solutions ${ }^{10}$. If this was unsuccessful, we contacted the corresponding author. If they did not reply within four weeks, we considered reproduction to have failed for this paper. Once the scripts compiled without issues, we did not further inspect the code or make any changes.

We documented all the technical issues and how we solved them. Each issue we encountered was categorized into one of the four categories: minor, substantial, severe, and system-dependent issues. All scripts that successfully compiled were then executed, and we saved all figures that they generated during execution. Figures consisting of several sub-figures (e.g. Figure a, b) were considered as one figure. The generated figures were then visually compared to the original ones in a side-by-side manner. Since figures (such as maps or time series) are frequently used to relay key results in academic papers, comparing those produced during reproduction to the figures in the original paper is one way to confirm that the reproduced results are identical to the reported ones. During this comparison, we recorded any differences that we found. Each type of difference, such as a label being different in the reproduced and original figure, was counted only once. This approach was chosen

\footnotetext{
${ }^{8}$ Copernicus Publications: https://publications.copernicus.org/, last access for this and the following URLs: 01.04.2019.

${ }^{9}$ Journal of Statistical Software: https://www.jstatsoft.org/index

${ }^{10}$ see e.g. StackOverflow: https://stackoverflow. com/
} 
to avoid issues with how to count different types of differences and to prevent over emphasizing consistent but repeated differences (e.g. numeric labels next to an axis all changing due to the depicted range being different in the reproduced and the original figure).

The supplemental material attached to this article includes a list of all papers that we examined as well as a reproducible RMarkdown document for Figure 3.2.

\section{Results}

Below, we first report on the technical issues we encountered and then summarize the differences between the original and reproduced figures that we observed.

Technical issues: The code of two papers ran without any issues, 33 had resolvable issues, and two were partially executable, i.e. the code produced output but also had issues that we could not resolve. We classified four papers as being unreproducible, as we could not solve all issues. The code of 15 papers contained issues that required contacting the corresponding author. Eleven authors helped us to find solutions, e.g. by pointing out code changes or solutions to the problems. Five of them sent additional code and data. One author helped with some but not all issues; three authors did not reply within the four-week time limit. In total, we encountered 173 issues in 39 papers (mean $\mu=4.4$ issues per paper), which we categorized as follows (see Table 3.2).

Minor issues were defined as being resolvable without any manipulation of the code that was provided by the authors. They mainly resulted from code calling a library that was not installed but could be found in public repositories, i.e. CRAN for $R$. This issue emerged 49 times in 24 papers (49/24). Minor issues also comprised negligible issues (4/3), e.g. a faulty function irrelevant for further computations. In total, we encountered 53 minor issues in 25 papers. Six papers only had minor issues.

Substantial issues $(73 / 25)$ required manipulating the code in order to resolve them, e.g. by adjusting file directories (34/13). We encountered deprecated functionalities (10/4) that had to be resolved by installing an archived version of the corresponding library or by using the current version with potentially deviating results. Substantial issues also resulted from functionalities which saved outputs locally but did not execute properly (10/2). These issues could be addressed by plotting the results within the programming environment. Further issues in this category were libraries that did not exist in the CRAN repository (8/7). Some scripts called functions without explicitly importing the library that provided these functions (9/4). Again, we had to find and embed the right library. The search was more demanding when the name 
Tab. 3.2: Issues we encountered during code execution. Numbers in brackets show how often and in how many papers they occurred (overall occurrence/number of papers). In total, we encountered technical issues in 39 papers.

\begin{tabular}{|l|l|l|l|}
\hline Minor (53, 25) & $\begin{array}{l}\text { Substantial (73, } \\
\text { 25) }\end{array}$ & Severe (41, 22) & $\begin{array}{l}\text { Sys.-dependent } \\
\mathbf{( 6 , 5 )}\end{array}$ \\
\hline $\begin{array}{l}\text { Library not found } \\
\text { but available in } \\
\text { repository }(49,24)\end{array}$ & $\begin{array}{l}\text { Wrong directory (34, } \\
13)\end{array}$ & $\begin{array}{l}\text { Flawed functionality } \\
(13,9)\end{array}$ & $\begin{array}{l}\text { Insufficient RAM } \\
(2,2)\end{array}$ \\
\hline $\begin{array}{l}\text { Faulty variable call } \\
(4,3)\end{array}$ & $\begin{array}{l}\text { Deprecated function } \\
(10,4)\end{array}$ & $\begin{array}{l}\text { Missing data or code } \\
(11,9)\end{array}$ & $\begin{array}{l}\text { Function behaves } \\
\text { differently across } \\
\text { OSes (3, 3) }\end{array}$ \\
\hline & $\begin{array}{l}\text { Output not storable } \\
\text { in local folder (10, 2) }\end{array}$ & $\begin{array}{l}\text { Flawed data integra- } \\
\text { tion (11, 8) }\end{array}$ & $\begin{array}{l}\text { Installing libraries } \\
\text { on different OSes } \\
(1,1)\end{array}$ \\
\hline & $\begin{array}{l}\text { Function not found } \\
\text { or missing library (9, } \\
\text { 4) }\end{array}$ & Code in PDF (6, 6) & $\begin{array}{l}\text { Library not found } \\
\text { and not in repository } \\
(8,7)\end{array}$ \\
\hline & Broken link (2, 2) & & \\
\hline
\end{tabular}

of the library was unknown, as different libraries can provide the same function name but with different implementations. We considered one reproduction as failed since we were unable to find an outdated library that provided the functions called in the script. Finally, in two papers the links to the required materials were broken, which meant that we had to contact the author and look for other repositories from the same author in order to access those materials.

Severe issues (41/22) required a deeper understanding of the source code and the programming language in order to be resolved. These issues arose when it was necessary to adapt functions or parameters (13/9) or when data or code segments were missing (11/9); while we were able to resolve the issues of one paper by ourselves, we had to contact the authors of the other eight papers to tackle the issues that emerged. Five authors sent the required material and one updated the library that had caused issues. Two authors did not reply within the four-week period, which meant that we classified the reproduction as failed. Further causes for severe issues included data not loading correctly (11/8) and having to extract code from a PDF (6/6), which entailed a number of copy-and-paste issues.

System-dependent issues (6/5) relate to problems resulting from the computational environment in which the code was run. Two analyses required more random access memory (RAM) than was available on the machine we used for our study. Some scripts (partially) failed when run inside a Docker container but worked fine 
Tab. 3.3: Number of design and content differences between original and reproduced figures. Bolded numbers in brackets show how many figures had the corresponding figure component. Italic numbers in brackets show in how many papers the differences occurred.

\begin{tabular}{|l|c|c|c|c|c|c|c|c|}
\hline $\begin{array}{l}\text { Compar- } \\
\text { ability }\end{array}$ & Total & $\begin{array}{c}\text { Legend } \\
\mathbf{( 3 8 )}\end{array}$ & $\begin{array}{c}\text { Labelling } \\
\mathbf{( 9 3 )}\end{array}$ & Results & $\begin{array}{c}\text { Aspect } \\
\text { Ratio }\end{array}$ & $\begin{array}{c}\text { Axes } \\
\mathbf{( 9 0 )}\end{array}$ & $\begin{array}{c}\text { Place- } \\
\text { ment }\end{array}$ & $\begin{array}{c}\text { Back- } \\
\text { ground } \\
\text { (50) }\end{array}$ \\
\hline Design & $\begin{array}{c}315 \\
(28)\end{array}$ & $\begin{array}{c}18 \\
(12)\end{array}$ & $60(20)$ & $44(19)$ & 78 & 64 & 18 & $33(9)$ \\
& & & $(25)$ & $(22)$ & $(11)$ & \\
\hline Content & $\begin{array}{c}105 \\
(27)\end{array}$ & $\begin{array}{c}15 \\
(11)\end{array}$ & $21(13)$ & $46(20)$ & N/A & $13(9)$ & N/A & $10(4)$ \\
\hline
\end{tabular}

(a)
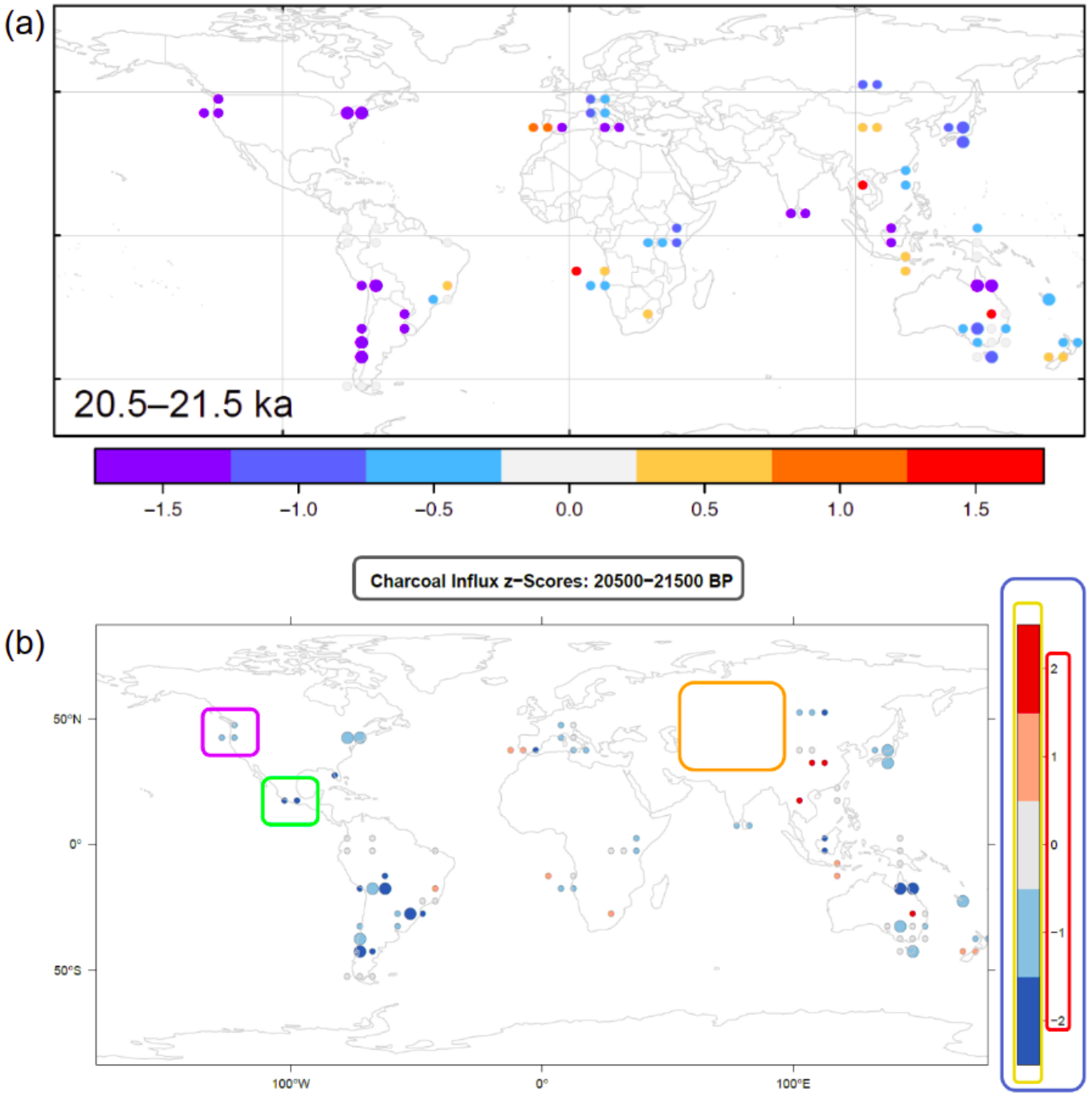

Fig. 3.2: Differences between the original (a) and reproduced figure (b) (Marlon et al., 2016). Design: placement of legend (blue box), aspect ratio, color of results (purple), data type of legend numbers (red). Content: labelling (grey), background map (orange), number of classes (yellow), deviating results (green). 
when we ran them on Windows (3/3). Finally, the installation of libraries might be different in a Docker container and on Windows (1/1).

Differences between original and reproduced figures: The code of 28 out of 41 papers produced 97 figures, which we compared to the ones contained in the original paper. We observed the following differences which impeded the comparability of figures and deviations between original and reproduced figures (Table 3.3).

Cosmetic differences: 78 out of 97 reproduced figures had a different aspect ratio than the original one. We encountered 44 cases where the visualization of the results differed regarding line widths or colors of bar charts and data points. In total, 90 figures consisted of diagrams that included axes, and 64 of those had a different font, interval, or data type (e.g. "2" instead of "2.0"). The axes were missing or had a different scale unit in a further 13 cases. We counted 50 figures that had a background (e.g. maps, grids). In 33 of these, the line widths of boundaries or grid structures differed, and in ten cases the level of detail was different, or the grid was missing entirely. A legend was present in 38 figures, and in 18 cases, it differed in terms of colors, font, or data types. Frequently, the legend was completely absent or incomplete (15 cases). Further differences we spotted relate to the placement of figure components (e.g. subfigures), which differed in 18 cases. Labels were present in 93 figures; we counted 60 cases with different fonts and 21 cases with a different or missing text. Overall, we counted 374 cosmetic differences that can affect the comparability of original and reproduced figures: 315 of those differences were related to the design of the figures, and 59 differences were related to the actual content of the figures.

Deep differences: The results of 46 out of 97 reproduced figures deviated from the original figures on a deeper level, e.g. graphs had different curves, and key numbers were missing or different. These differences make it harder for readers to determine whether or not the reproduced figures depict the same results as those shown in the original figures. We did not find systematic correlations between the specific issues, cosmetic differences discussed, and deeper differences. In the case of deeper differences, confirming successful reproduction will thus most likely require a more in-depth inspection, e.g. using raw and/or intermediate results produced by the scripts.

In order to illustrate the differences and the resulting difficulties when comparing an original and a reproduced figure, consider the following example from Marlon et al. (2016). The different aspect ratio leads to a different appearance that might be interpreted as the results being different although the actual numbers from the original and the reproduced analysis are identical. Figure 3.2 shows a real example in which we highlighted typical differences in the reproduced figure. We are very 
grateful to the corresponding author (Marlon et al., 2016) for permitting us to include their figure as an example to illustrate typical differences that can occur during reproduction.

\subsection{Discussion}

The main goal of the work presented in this article was to shed light on how reproducibility is perceived and practiced in the computational geosciences. In our studies, geoscientists had divergent perceptions of what ORR means. Half of the survey participants said they published re-computable research results, but they rarely linked to source code and data underlying the figures and numbers. Interviewees already conducted computational research such as spatial statistics but focused mainly on the methodology and associated it with replicability (Bollen et al., 2015). As a detailed description of the methodology can be sufficient to ensure replicability, this might explain the high number of respondents claiming that they publish reproducible research though many did not attach code and data. Three interviewees initially struggled when asked to define the term, which might indicate that the topic is not fully present in their daily work. The low number of geoscientists who regularly reproduce other researchers' work (7\%) confirms this impression. The fact that only a few of them succeeded confirms the findings reported by Baker (2016a).

The obstacles hindering $O R R$ mentioned by interviewed geoscientists also confirm what has been reported in the literature for other disciplines (cf. Baker, 2016a). It seems some of the obstacles impede both authors and readers, such as the use of proprietary software preventing code publication and reproduction. The results from the questionnaire and the interviews indicate that realizing openness is one key issue in reproducible research, which provides support for the current trend towards openness in science in general (open access, open data, open code).

While executing the code of the papers, we encountered a substantial number of technical issues and observed various differences between original and reproduced figures. The code of only two papers was executable without any issues. 33 out of 41 papers were executable after we resolved different types of issues that varied in terms of severity and effort required to address them. We also came across a number of system-dependent issues that resulted from implicit assumptions about the underlying system (e.g. the operating system). These issues might highlight the importance of describing the computational environment in reproducible research. Reproducibility in general hinges on being able to produce the results from the paper. The figures showing results (e.g. maps) provide an effective way to quickly compare original and reproduced results. In our study, we identified numerous cosmetic and 
deeper differences between the figures in the original publication and the figures that were generated during reproduction (see Figure 3.2 for an example). It was not straightforward to determine whether results deviate because reproduced figures had a different aspect ratio or because the computational steps produced a different outcome. Finding the right configuration (e.g. parameters) to produce identical figures usually requires effort and knowledge of the code. These figure-related issues, as well as the technical ones mentioned above, provide initial evidence that "just" making code and data publicly available often does not guarantee that others can execute the analysis and produce identical results.

Besides code, it is still important to consider data underlying a geospatial analysis. Ideally, authors should provide their geographic data following the FAIR principles (Wilkinson et al., 2016): Data should be findable, i.e. by persistent identifiers; accessible, i.e. for free; interoperable, i.e by using open formats (e.g. GeoJSON instead of shapefile); and reusable, i.e. by using open licenses. Several other approaches might counteract the obstacles in ORR. Educating graduate students, offering workshops for scientists, or conducting hands-on seminars addressing the technical difficulties while publishing reproducible results might be promising solutions (cf. Leek and Jager 2017). Such educational initiatives could increase the awareness of ORR not only for one's own research but also while reviewing others' submissions.

Making available tools that integrate well with geoscientists' existing workflows and that provide support for open reproducible work might be another key element for boosting reproducibility in the geosciences. Some initial proposals have been made in this field (see chapter 4), and the common domain of space and time holds great potential in this respect. Geographic information systems, satellite imagery, and geospatial analysis systems (such as libraries for geospatial statistics in R) are widely used throughout the geosciences. Integrating those systems and datasets into reproducibility tools may drastically lower the effort needed to work reproducibly while keeping in line with geoscientists' existing workflows.

While it is highly desirable and necessary to address technical issues, provide supportive tools, and further educate geoscientists about $O R R$, these steps alone are probably not sufficient to eliminate the practice of publishing unreproducible research. The overall culture, processes, and reward systems around scientific work need to be adjusted, too. Scientists (including but not limited to those who conduct computational research) need to shift towards ORR in their working methods (Markowetz, 2015). In addition, fears and worries of (geo-)scientists need to be addressed. For example, we observed that some authors were reluctant to fully disclose their own work since they feared that others would either "steal" it or find issues that might damage their reputation. More fine-grained citation systems (e.g. for data, code) and 
"evolving" publications (e.g. where credits are given to improvements proposed by researchers other than the authors) might be ways to deal with such fears. In addition, there is the issue of a lack of incentives to reproduce other researchers' work. If reviewers would receive rewards for their effort to reproduce submissions (Stodden et al., 2016), some issues could already be resolved during the review process and reproduction could become more widely practiced. Furthermore, publication outlets might consider desk-rejecting submissions that are not reproducible.

Limitations: The work presented in this article aims to shed light on how ORR is perceived and practiced in the computational geosciences. In order to keep the scope of the research manageable, we made several assumptions and decisions which limit the generalizability of our results. One key limitation pertains to the study participants. The number of participants and their research areas do not represent the diversity of the geoscientific domain. Moreover, interviewees and focus group participants were recruited from the same faculty. Researchers from other institutions or countries might hold different perceptions of ORR or may be able to identify other obstacles. Although we contacted scientists from several geoscientific domains to complete the survey, the total number of respondents (146) still does not represent all geoscientists. Consequently, some research domains within the geosciences might not have been included in our studies. In addition, it is also likely that the survey was completed by people who are inherently interested in reproducible research, which thus could have introduced some bias. A truly representative selection from all geoscientists and all geoscientific domains is difficult to achieve.

Another limitation pertains to the selection criteria for papers we used in the reproducibility study. Due to practical reasons (e.g. the authors' familiarity with certain programming languages and tools) as well as time constraints, we focused on very recent papers from few outlets published by Copernicus and the Journal of Statistical Software. While this decision enabled us to systematically evaluate the reproducibility of the selected papers, it also makes the findings less representative. However, in recent years, new technologies have emerged that assist scientists in sharing code and data (e.g. GitHub and Zenodo). Hence, recent papers likely do show the best current practice, and older papers might show even worse results. More papers from other outlets are needed to draw informed conclusions. In addition, we only focused on computational reproducibility rather than general reproducibility. While this limits the scope of the article, we argue that computational reproducibility is particularly relevant in the geosciences since many papers include results that are produced by source code. Although the identified technical issues were specific to the $R$ programming language, it seems very likely that similar issues might also emerge when using other programming languages (such as Python, which is also very popular in the geosciences). In order to confirm this, we plan to run another 
study with articles based on Python to see if similar or different issues emerge and with what frequency.

Despite us defining ORR explicitly in the studies, participants might still have had replicability in mind while answering the questions, which might constitute another limitation. Since we conducted the studies in German and English, we took great care to provide identical definitions of $O R R$ in both languages. Though in principle translation issues might constitute another limitation, we consider this unlikely. Finally, we did not investigate the authors' intentions behind attaching materials, i.e. whether they did it to facilitate reproduction of the results or just as supplements. This aspect could explain some of the issues, differences, and deviating results. If authors anticipate that readers want to reproduce their work, they might take greater care when preparing data and code than when authors only include material for completeness or to comply with a journal's requirements.

\subsection{Conclusion}

Reproducibility is essential to computational research in general and consequently to papers that apply spatial statistics based on code and data. In order to shed light on ORR in the computational geosciences, we reported on a series of studies that examined issues, perceptions, and practices related to ORR. We conducted an online survey (146 responses), semi-structured interviews $(n=9)$, a focus group $(n=5)$, and a reproducibility study with 31 articles including spatial statistics published by Copernicus Publications and ten papers from the Journal of Statistical Software. Our main contributions are the initial identification of (1) geoscientists' understanding of the term ORR and (2) practical obstacles which might hinder reproducibility in the computational geosciences. Moreover, we report on (3) issues arising when reproducing papers that apply spatial statistics based on code and data, and (4) differences in the reproduced results. Our final contribution is the provision of (5) a set of guidelines for authors wishing to publish reproducible work.

The results from our studies indicate that geoscientists might have a divergent understanding what reproducibility means, and that reproducibility might be hindered by a lack of openness regarding data, code, and proprietary software. In order to gain direct insight into current practice, we tried to execute the source code attached to 41 scientific publications and analyzed the figures that were generated during reproduction. In total, we identified 173 issues, which we classified into four categories: minor, substantial, severe, and system-dependent issues. We compared 97 original and reproduced figures and detected 420 differences, which were either cosmetic or of a deeper nature. It appears that publishing code and data with a paper does not guarantee that the reported results can be easily reproduced and 
that the figures generated during reproduction are identical to those in the original paper. To overcome the issues, we propose the following guidelines for publishing computational research reproducibly:

1. Embed and install libraries within the source code and include their version numbers to facilitate finding the right libraries.

2. Make directories relative to a top directory (see here ${ }^{11}$ package for $R$ ) instead of the author's computer.

3. Do not modify the source code once the results are copied into the paper, as later changes of the code might affect already extracted results.

4. Execute the code in a clean programming environment after completing the analysis, e.g. by using rocker/geospatial, to spot issues other readers might have.

5. Publish input data and processed data of figures to enable readers to assess whether deviating figures result from differences in the data analysis or in the settings of the systems used to generate the original and the reproduced figures.

6. Encapsulate code and data in a project folder to facilitate execution (Fehr et al., 2016), e.g. as an Executable Research Compendium (see chapter 4).

7. Provide code and data in original files instead of PDFs to avoid cut-and-paste issues and to lower the burden for readers to re-run and reuse the analysis.

8. Use code to produce and design figures (Sandve et al., 2013), ideally embedded in executable documents, e.g. RMarkdown (Allaire et al., 2018) or Jupyter notebooks (cf. Kluyver et al., 2016), to avoid scaling issues.

Our recommendations confirm and complement suggestions regarding reproducible computational analyses in general (Bailey et al., 2016) but were specifically designed to address the issues we encountered when reproducing the 41 selected papers. The recommendations could also be used as author guidelines in conferences and journals. In addition, they might inform the design of tools that support working reproducibly.

${ }^{11}$ here package: https : //www.r-bloggers.com/making-an-r-package-to-use-the-here-geocode-api/, last access: 01.04.2019. 


\section{Acknowledgement}

We are very grateful to Marlon et al. (2016) for publishing open and reproducible research and for allowing us to use their figure as an example in this paper.

\section{Supplemental material}

An "Open Science Framework" project with code and data is accessible under https: //osf.io/b2n78/?view_only=7f67dc2f25e348ebbb6c584ace93ce51 (last access: 01.04.2019). 


\title{
Opening the publication process with executable research compendia
}

\author{
This chapter was published as: Konkol*, Markus, Daniel Nüst*, Edzer Pebesma, Chris- \\ tian Kray, Marc Schutzeichel, Holger Przibytzin, and Jörg Lorenz (2017). “Opening \\ the publication process with executable research compendia". In: D-Lib Magazine, \\ 23(1/2). eprint: http://www.dlib.org/dlib/january17/nuest/01nuest.html. \\ It corresponds to P1 in the list of publications.
}

*Shared co-first authorship

\begin{abstract}
A strong movement towards openness has seized science. Open data and methods, open source software, open access, open reviews, and open research platforms provide legal and technical solutions to new forms of research and publishing. However, publishing reproducible research is still not common practice. Reasons include a lack of incentives and a missing standardized infrastructure for providing research material such as datasets and source code together with a scientific paper. Therefore we first study fundamentals and existing approaches. On that basis, our key contributions are the identification of core requirements of authors, readers, publishers, curators, as well as preservationists and the subsequent description of an executable research compendium (ERC). It is the main component of a publication process providing a new way to publish and access computational research. ERCs provide a new standardizable packaging mechanism which combines data, software, text, and a user interface description. We discuss the potential of ERCs and their challenges in the context of user requirements and the established publication processes. We conclude that ERCs provide a novel potential to find, explore, reuse, and archive computer-based research.
\end{abstract}

\subsection{Introduction}

Open access is not only a form of publishing that ensures research papers become available to the broad public free of charge, but it is also connected to a trend towards "open science", which makes research more transparent (Nosek et al., 
2015), and see also Charles W. Bailey, What is open access? ${ }^{1}$ and open access to Scientific Information, History of the Open Access Movement ${ }^{2}$ ). To fully realize open (computational) science, we expect everyone can reproduce findings because access is granted to papers, research data, methodology, and the computational environment. In parallel, the scientific paper "is evolving into a multi-part distributed object" that can include the paper, data, and code (Hanson et al., 2015, p. 1). Access to these components is rarely given, making it challenging to archive and reproduce methods and results. Reasons include privacy concerns and copyright issues. Another key obstacle is the lack of standardized means for sharing all the parts of an "evolved" scientific paper in a natural way (Hanson et al., 2015).

The main contribution of this work is the definition of a compendium-based publication process. It facilitates "open science" and enables new ways to conduct research. Its core component is the executable research compendium (ERC), which opens and integrates the scientific process across all activities and stakeholders. The compendium includes, besides the actual paper, source code, the computational environment, the dataset, and a definition of a user interface. It has internal connections which facilitate new ways of interacting and reuse. Such a compendium is a self-contained entity and can be executed almost entirely on its own, requiring only a generic virtualization environment. ERCs are intended for the huge number of research projects which live on a researcher's desktop computer. Larger scale undertakings with high computational or storage requirements, e.g. distributed infrastructures, and using external third-party services ("black boxes") are out of the scope of this work although being subject to vivid research (cf. Chen et al., 2016; Goecks et al., 2010).

The paper is structured as follows. The next section provides a review of related work and basic concepts. In the central part, we explain how the ERC builds upon stakeholder requirements and current technologies. We describe the process of publishing ERCs and conclude by highlighting the main contributions and future work.

\subsection{Related Work}

In this chapter, we introduce the term "reproducibility" and open access as motivators of an open publication process. Our work builds on the concept of research compendia/objects, data platforms, and methods to capture software environments. We also summarize key challenges in open reproducible research.

\footnotetext{
${ }^{1}$ What is open access: http://digital-scholarship.org/cwb/WhatIsOA.htm, last access for this and the following URL: 01.04.2019.

${ }^{2}$ History of open access: https://open-access.net/DE-EN/information-on-open-access/ history-of-the-open-access-movement/
} 


\subsubsection{Definition of Reproducibility}

In the current research, talking about computational reproducibility can lead to confusion as a universally accepted definition of the term "reproducibility" is not yet established. While some researchers treat "reproducibility" and "replicability" as interchangeable terms (Bollen et al., 2015), others distinguish them carefully (Leek and Peng, 2015; Goodman et al., 2016). "Replicability" is given if researchers receive the same results by using the same methodology but a different dataset (Bollen et al., 2015). Research findings are "reproducible" if researchers can compute the same results by using the same procedure and the same dataset (Bollen et al., 2015). This means a researcher requires the entire set of information which constitutes the basis of the results reported in the paper including datasets, source code, and configuration details (Vandewalle et al., 2009). A key difference between the two terms is the dataset which needs to be different from the originally used dataset in the case of "replicability", and to be the same in the case of "reproducibility". Consequently, it does not mean that reproducible research findings are valid. They can still be subject to flaws in the study design (Leek and Peng, 2015). However, reproducible outcomes are more reliable as they allow other researchers to understand the (computational) steps described in the paper. In open reproducible research, the components required to reproduce the results are publicly available. Other scientists are thus able to reuse parts for their research.

\subsubsection{Open Data and Open Access}

Today, most researchers find it difficult to reproduce the analysis reported in papers published 5-10 years ago, or even recover the data. For instance, Vines et al. (2014) reported a half-life of four years for data recovery from the original authors. Nowadays there is a strong trend towards publishing scientific papers as open access, meaning anyone has free access to the published material, including free access to data and software (cf. The Open Definition ${ }^{3}$ : "Open data and content can be freely used, modified, and shared by anyone for any purpose."). Different models exist (cf. Harnad et al., 2004) and publishers increasingly offer open access routes for research papers. For instance, PLoS-One's policy requires authors to publish data by default (Bloom et al., 2014). The Directory of Open Access Journals (DOAJ ${ }^{4}$ ) lists over 9000 journals. Open access is a key topic in the European Union's research programme Horizon $2020^{5}$. Dissemination and reuse of scientific findings shall be

\footnotetext{
${ }^{3}$ The Open Definition: http://opendefinition.org/, last access for this and the following URLs: 01.04.2019.

${ }^{4}$ DOAJ: https://doaj.org/

${ }^{5}$ Horizon 2020: https://ec.europa.eu/programmes/horizon2020/en/h2020-section/ open-science-open-access
} 
improved by making them accessible at no charge through suitable e-infrastructures (European Commission, 2015) such as the "European Open Science Cloud"6.

\subsubsection{Research Compendia and Research Objects}

Gentleman and Lang (Gentleman and Lang, 2007) use the term Research Compendium (RC) to refer to the unit of scholarly communication which includes the research paper, the code, and the data with a "dynamic document" (cf. Knuth, 1984) at the core. They present the advantages and potential uses of (executable) research compendia, which allow to completely reproduce the computational aspects of a scientific paper. (Randomness of specific simulations or machine learning methods can be handled by setting a seed. Numerical differences between runs can be mitigated by appropriate rounding. Both these aspects require careful design of the actual analysis and are beyond the scope of ERC.) The practical implementation focuses on "single language compendia", which are essentially the respective languages' standard packaging mechanism (e.g. $R$ packages). Stodden et al. (2015) developed a platform for sharing, executing and validating Research Compendia.

Research Objects (RO) are "semantically rich aggregations of resources that bring together the data, methods, and people involved in (scientific) investigations" (Bechhofer et al., 2013, p. 609). They comprise metadata standards and bundling of any kind of resource across a range of scopes, such as scientific workflows (Belhajjame et al., 2015), preservation (Research Object BagIt archive ${ }^{7}$ ), or computational jobs ${ }^{8}$. These can be included or remote (i.e. linked) resources. ROs are also Linked Data resources themselves and can be managed and preserved in a tailored platform (Palma et al., 2013).

Both Nature and Nature Geosciences published about the need to publish reproducible code (Nature Editorial, 2014; Easterbrook, 2014). The majority of scientific journals allow adding supplementary material to publications, and hence creating and publishing Research Compendia or Research Objects has been possible for a long time. Still, current supplemental material frequently looks very much like a directory on the researcher's personal computer.

\footnotetext{
${ }^{6}$ European Open Science Cloud: http://ec.europa.eu/research/openscience/index. $\mathrm{cfm}$ ?pg= open-science-cloud, last access for this and the following URLs: 01.04.2019.

${ }^{7}$ BagIt: https://github.com/ResearchObject/bagit-ro

${ }^{8}$ Computational jobs: http://www.researchobject.org/initiative/stelar/
} 


\subsubsection{Research Data Platforms}

We see a large number of technical solutions for research repositories, including RunMyCode $^{9}$, the Open Science Framework ${ }^{10}$, Zenodo $^{11}$, and figshare ${ }^{12}$ (see Dave Wilkinson's "rubric"13 for platforms). All these platforms provide identification (some only by email; others support ORCID ${ }^{14}$ or GitHub ${ }^{15}$ identities), uploading, and persistent identifiers for citation of deposited items. All platforms have a data size limitation, some have paid upgrade plans. Some sites argue users should trust their long-term availability, others offer the service as-is, for example, RunMyCode is provided on an "as is" and "as available" 16 basis. All these platforms are fine for viewing and downloading, but none of them performs the computations needed to carry out reproducible research. This is most likely due to re-computation requiring considerably more resources than storage, and thus causing substantial costs, and security concerns. Also, the platform would need to understand a plethora of different computational setups and would have to cater for the execution of compendia within these.

\subsubsection{Capture Workflows and Runtime Environments}

Researchers enjoy conducting their research in an environment composed of software of their own choice. Consequently, the number of unique research environments approximates the number of researchers, and hence expecting one standardized computational workflow would not be feasible. However, the complete computational environment of the original researcher, the runtime environment, is an essential aspect of reproducibility. Different approaches to capture the workflows and runtimes can be chosen.

Regarding workflows, a commonality of all computational sciences is carrying out a number of steps (process input file(s), process data into the result, e.g. number or graph). These steps are ordered and must be documented to allow understanding, execution, and reproduction. The canonical example is the classic unix utility "make" (Mecklenburg, 2004), which executes commands according to the instructions in a Makefile. The concept has been adapted for specific scenarios, e.g. remake ${ }^{17}$ for data analysis in $R$, drake ${ }^{18}$ for data workflow management, or Taverna (Wolstencroft

\footnotetext{
${ }^{9}$ RunMyCode: http://www.runmycode. org/, last access for this and the following URLs: 01.04.2019.

${ }^{10}$ OSF: https://osf.io/

${ }^{11}$ Zenodo: https: //www . zenodo.org/

${ }^{12}$ figshare: https://figshare.com/

${ }^{13}$ rubric: http://wilkie.github.io/reproducibility-site/rubric.html

${ }^{14}$ ORCID: https://orcid.org/

${ }^{15}$ GitHub: https : //github.com/

${ }^{16}$ as is, as available: http://www.runmycode.org/terms-of-use.html

${ }^{17}$ remake: https://github.com/richfitz/remake

${ }^{18}$ drake: https://github.com/Factual/drake
} 
et al., 2013) for web-based workflows. Santana-Perez et al. (2017) demonstrate a semantic modeling approach to conserve scientific workflow executions based on semantic vocabularies. Thain et al. (2015) discuss two broad approaches to capture scientific software executions: "preserving the mess, and encouraging cleanliness" They provide an extensive picture on technical and organizational challenges in the context of in-silico experiments and present prototypical solutions (e.g. Umbrella).

Alternatively, the environment can be confined to an interpreter of a particular language, such as $R$ (R Core Team, 2018). The environment is reproduced if the correct versions of the interpreter and all extension packages/libraries/modules are used. These dependencies quickly become complex, so manual documentation is not feasible. The $R$ extension packages checkpoint ${ }^{19}$ or packrat $^{20}$ ease the process of reproducing project dependencies, except the interpreter itself. ReproZip (Chirigati et al., 2013) or Parrot (Thain et al., 2015) apply tracing techniques to capture the minimal set of objects and commands needed for the analysis and use it to build a virtual machine (VM) or container image for execution.

Containerization originates from packaging applications and their dependencies for deployment in cloud infrastructures (cf. Dua et al., 2014). Containers have proven to be a suitable technology in reproducible research: Howe (2012) lists improvements virtualization and cloud computing provide for reproducibility, all of which apply directly to containerization. Boettinger (2015) demonstrates its usage for computational analysis in $R$ and derives best practices. Marwick (2015) accompanies a research article by Clarkson et al. (2015) with a complete research compendium containing the analysis (also available on GitHub ${ }^{21}$ ). Hung et al. (2016) use containers to package graphical user interface-based research environments with multiple tools from multiple languages across operating systems in the context of bioinformatics.

The dominant platform for containerization is Docker (Merkel, 2014). Its images are created using a recipe called Dockerfile - a Makefile with inheritance support for creating containers. Images can be built and executed on any machine running a Docker host, and are distributed in a readily executable form via online hubs, for example, Docker Hub ${ }^{22}$ or Quay ${ }^{23}$.

\footnotetext{
${ }^{19}$ checkpoint: https://cran.r-project.org/web/packages/checkpoint/index.html, last access for this and the following URLs: 01.04.2019.

${ }^{20}$ packrat: https://cran.r-project.org/web/packages/packrat/index.html

${ }^{21}$ GitHub: https://github.com/benmarwick/1989-excavation-report-Madjedbebe

${ }^{22}$ Docker Hub: https ://hub. docker. com/

${ }^{23}$ Quay: https: //quay.io/
} 


\subsubsection{Challenges in Open Reproducible Research}

Solutions to technical and legal challenges concerning implementing reproducible research exist (cf. Stodden et al., 2014). Other challenges, such as the question of incentives and developing reward mechanisms, are closely connected to the intricacies of academic authorship and credit. The challenges and some approaches are summarized in the Wikipedia article on academic authorship ${ }^{24}$, for example, the individual h-index ${ }^{25}$ (George A. Lozano) or Nature's author contributions statements ${ }^{26}$.

Among the reasons why researchers do not publish reproducibly, Borgman (2007) mentions (1) a lack of incentives in terms of citations or promotion, (2) the effort required to clean data and codes, (3) the creation of a competitive advantage over other fellows, and (4) intellectual property issues. Other reasons include privacy or confidentiality issues (Glandon, 2011). None of these issues can be solved by technical solutions alone. Instead, they require a discourse within the scientific community, or even a mind-shift to "build a culture of reproducibility" based on selfish reasons to publish reproducibly (Markowetz, 2015, p. 3).

Although moving the relevant parts of a researcher's hard drive to public and citable archives is a step forward in the direction of open access to research findings, we believe this alone will not convince researchers to adopt it as standard practice. Such archived workspaces could be incomprehensible (Easterbrook, 2014). The potential reward is considered smaller than the possible reputation damage or expected follow-up work, e.g. support questions.

Each scientific community must address such worries to make publication of workspaces an effective incentive for reproduction. Encouraging statements such as Nick Barnes' (2010) column "Publish your computer code: it is good enough" pave the way towards venturing reproducibility of software. However, they also highlight the necessity for communication of researchers' uncertainties regarding reproducibility as a deliberate act of exposure to critics and competitors.

The research data repositories mentioned before support reproducibility, but they cannot carry out the actual reproduction, nor do they suggest conventions for doing so in an automated fashion. Although they are designed to deposit code and data supplementing a scientific paper, none of them require documenting the runtime environment, under which the reproduction material is expected to reproduce the paper systematically. This puts more burden on the original author, as each is

\footnotetext{
${ }^{24}$ Academic authorship: https://en.wikipedia.org/wiki/Academic_authorship, last access for this and the following URLs: 01.04.2019.

${ }^{25}$ h-index: https : //arxiv .org/abs/1307.1330

${ }^{26}$ Nature policies: https ://www. nature.com/authors/policies/authorship.html
} 
supposed to know how to describe this generically. The same burden is placed on a reader as the reproducer.

\subsection{The Executable Research Compendium}

\subsubsection{Stakeholders and Roles in the Publication Process}

The ERC brings together perspectives of different user groups involved in scientific publications. To develop a common ground for them, we first consider some essential requirements of users separately. We identified these requirements in discussions including experts from university, library, and publishing (members of the project team and external partners ${ }^{27}$ ).

Authors must be given support to create an ERC without too much additional work, ideally starting from their digital workspace "as is". Since filling out forms for metadata is often perceived as a daunting and time-consuming task, an ERC should be restricted to a minimal set of required metadata. Ideally, the needed information can be (semi-)automatically deduced via existing identifiers and catalogs, such as DataCite (Brase, 2009) for datasets and ORCID (Haak et al., 2012) for researchers, thus avoiding the need for entering them multiple times. If common practices for reproducible research are followed then creating an ERC should be as simple as manually editing a configuration file and running a single command.

Readers want to be able to re-run the analysis in an ERC. On a basic level, a compendium must provide a simple "start" button to run an analysis and a trafficlight-like signal to learn about the result, cf. Elsevier's Earth and Planetary Innovation Challenge winning submission "One-Click-Reproduce" (Pebesma, 2013). A green light shows a successful, a red one an unsuccessful reproduction of the results. Here a green light means the computational steps are executable and the results are equal to those submitted by the original author. A red light means this is not the case. Either way, the status light is no indication on the validity of the findings. At an advanced level, readers can examine detailed information on, for example, data and code underlying figures, or errors preventing successful execution. A typical reader's question might be about assumptions and concrete tools the authors did not mention. Moreover, it would be interesting for readers to see how the results change and if the conclusions still hold after manipulating parameters.

Reviewers must be put in the position to scrutinize a piece of research. While potential malicious intent of the submitting authors is not considered here, review processes

\footnotetext{
${ }^{27}$ project team and partners: https://o2r.info/about/, last access: 01.04.2019.
} 
still involve a trust component as far as scientific and scholarly best practices are concerned. Reviewers often have to rely on the analysis in a research paper to be complete, correct, and consistent with the included visualizations. In order to verify the analysis, they need tools to re-create computation-based analyses with minimal effort. As a review and publication process is well established, the ERC shall align itself with it. It becomes the item under review and should help to increase the quality of the review. Since this also increases the quality of journals, ERCs ultimately serve the needs of editors, authors, readers, and publishers.

Libraries and publishers make research articles accessible to the scientific community and the public. However, access to the source material is not always granted. One of the reasons for this is neither libraries nor researchers are currently familiar with a publication process that allows for including procedures used to produce research outcomes (tables, figures). Any new tool for this purpose must leverage their expertise and pay attention to their existing workflows.

Curators and preservationists require the reader's basic level to assess a compendium's status. A compendium run must entail an execution of the contained computation and an automatic validation of the result to ensure the integrity of the digital asset. Since they need to integrate an ERC into a curation workflow, the required metadata must follow standards for digital preservation. As they cannot rely on the execution platform to be persistent, they need access to relevant metadata in "their" formats independently from the data and software within the compendium. Subsequently, the top-level package of the compendium should follow a preservation standard.

To fulfill the needs of these different user groups, we define conventions in the remainder of this chapter. They allow to create research compendia supporting automatic execution and to create services for executing such research compendia.

\subsubsection{Core Parts of ERC}

The core parts of an ERC are data, software, documentation, and user interface (UI) bindings (see Figure 4.1).

Data comprises all inputs for an analysis, ideally starting with raw measurements, for example, in the form of text files, or databases.

Software comprises code created by a researcher and all underlying libraries or tools to reproduce the analysis in the form of scripts/source code, a Dockerfile, and a Docker container. 


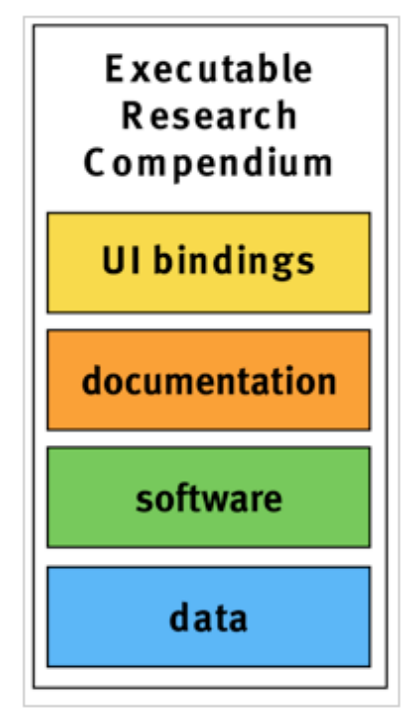

Fig. 4.1: The core parts of an ERC are data, software, documentation, and UI bindings.

Documentation comprises both instructions, such as a README file, and the actual scientific publication, e.g. in PDF format, any supplemental records, and metadata in standardized formats. The actual publication is the main output of the compendium and the core element for validation. An important metadata element is a license for each part of a compendium.

UI bindings provide linkage between research components and user interface widgets. They can be used to attach UI widgets to static diagrams in order to make them interactive. Their representation can be stored as metadata within an ERC as part of the documentation. The resulting UI widgets open up the container and allow readers to drill deeper into results. UI bindings can unveil parameters which are required for a comprehensive understanding but are often buried in the code.

\subsubsection{Creation and Reproducibility}

Two approaches for creating an ERC are possible: post-hoc or on-the-fly. Post-hoc creation is based on the regular workspace, which is a collection of files (data, code, documentation). Authors of a publication can submit a workspace to an ERC building service to generate a compendium. Such a service requires complex logic and user intervention, either by an expert on the service provider side or by the author, to detect how to start the analysis and how to validate its result. Following best practices from reproducible research, such as literate programming (Knuth, 1984) or having a default main file and execution command (comparable to a Makefile), can considerably reduce the required intervention. An ERC specification can support this with a "convention over configuration" approach (see Wikipedia, 
Convention over configuration ${ }^{28}$, Nicholas Chen, "Convention over Configuration"29, and also Maven standard directory layout ${ }^{30}$ ). Information that cannot be derived automatically must be elicited during the submission process, ideally with pre-filled forms.

Alternatively, the creation and maintenance of a compendium happen on-the-fly while carrying out research. A user starts with a template compendium. Since the compendium evolves with the work in progress, it can be executed and checked regularly. In both cases, the ERC is bound to a specific publication document. This "exit point" is also well-defined in the ERC and is exploited for result validation and to highlight changes in the results after parameter manipulation.

In order to reproduce an analysis, we make use of the containerization technology Docker. Storing both a docker image and the corresponding Dockerfile creates two levels of reproduction: (i) the ability to provide the original runtime environment, and (ii) re-creating it from scratch, potentially in a more recent version. Docker mitigates some issues for the reproduction service to run the software of the original researcher, for example, with a varying environment of the workspace (Linux, Windows, OS-X), but cannot solve others, for example, requiring licensed software $\left(\right.$ MATLAB $^{\mathrm{TM}}$, ArcGIS $^{\mathrm{TM}}$ ) which must not be redistributed.

Existing approaches using Docker to replicate environments (see the previous chapter) require readers to follow individual written instructions and to have expertise in the used software setup. This is not apt for a one-click execution (for readers) nor automatic content validation (for reviewers and curators). The ERC defines machine-readable conventions for computer systems to control and evaluate the embedded container, namely command-line interface instructions to run it (e.g. "docker run" as part of ERC metadata), and rules to check a successful execution based on the created workspace. The Dockerfile used to build this container defines the environment and command for analysis execution.

After an ERC's execution, the result is evaluated. A minimal evaluation relies on the exit code ${ }^{31}$ of the main process in the container. At an advanced level, checksums or contents of files and execution logs can be evaluated.

Once the creation service can execute the analysis, the information accessible at runtime, e.g. loaded modules and libraries as well as dataset objects, is a fast and

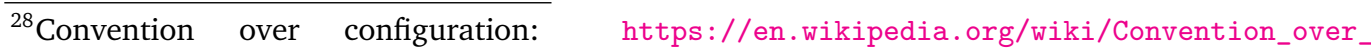
configuration, last access for this and the following URLs: 01.04.2019.

${ }^{29}$ Convention over Configuration by Nicholas Chen: http://softwareengineering.vazexqi.com/ files/pattern.html

${ }^{30}$ Maven: https://maven . apache.org/guides/introduction/

${ }^{31}$ Exit code: http://tldp.org/LDP/abs/html/exitcodes .html\#EXITCODESREF
} 
reliable way to derive both software metadata and dataset metadata. The potential information stretches from full names and versions of attached libraries to the spatial and temporal extent of the data subsets. This is prone to be more reliable than analyzing source code, using system-wide installed software, trying to read all possible data formats while assuming all data files in a workspace are used, or manual documentation.

Docker is well suited for storing and transporting software and its dependencies, but it does not serve the needs of data repositories and archives well, that are concerned with bitstream preservation and integrity of files.

\subsubsection{Publication Process}

An ERC facilitates a scientific publication that is not only composed of a paper but also the data, the source code, and the software environment, packaged in an executable manner. The executable research compendium described in this work is a fundamental component of the proposed publication process consisting of four consecutive steps (see Figure 4.2). The process is aligned with peer-review based journals. Their existing practices and protocols can be applied for transfer of compendia between stages and handling the required data, such as related communications or the state within the process.

In the first sub-process, the author prepares the research material as described in the previous section resulting in an unvalidated research compendium (URC). A URC is neither executable, not necessarily visible to the public, but it ideally contains already the entire research material including dataset, source code, and the software environment. A URC forms the input to the validation sub-process which involves (i) metadata verification, (ii) the error-free execution of the reproducible parts of the paper, and (iii) a confirmation from the primary author that the automatically reproduced results are identical to the actual publication. The first two aspects should be automated as part of a submission. If the validation succeeds, it produces an executable research compendium (ERC), which is passed on to the next subprocess.

The review process focuses on human inspection. It is necessary to avoid publishing meaningless or questionable compendia. Human inspection can be done by library staff (e.g. to ensure adherence to non-formal criteria), maintainers of a data repository (e.g. to filter offending or illegal content), journal editors, and peer reviewers (e.g. to warrant scientific quality in the context of a journal submission). If the review process has a positive outcome, it produces a reviewed executable research compendium (RERC), which constitutes the input to the final sub-process: 


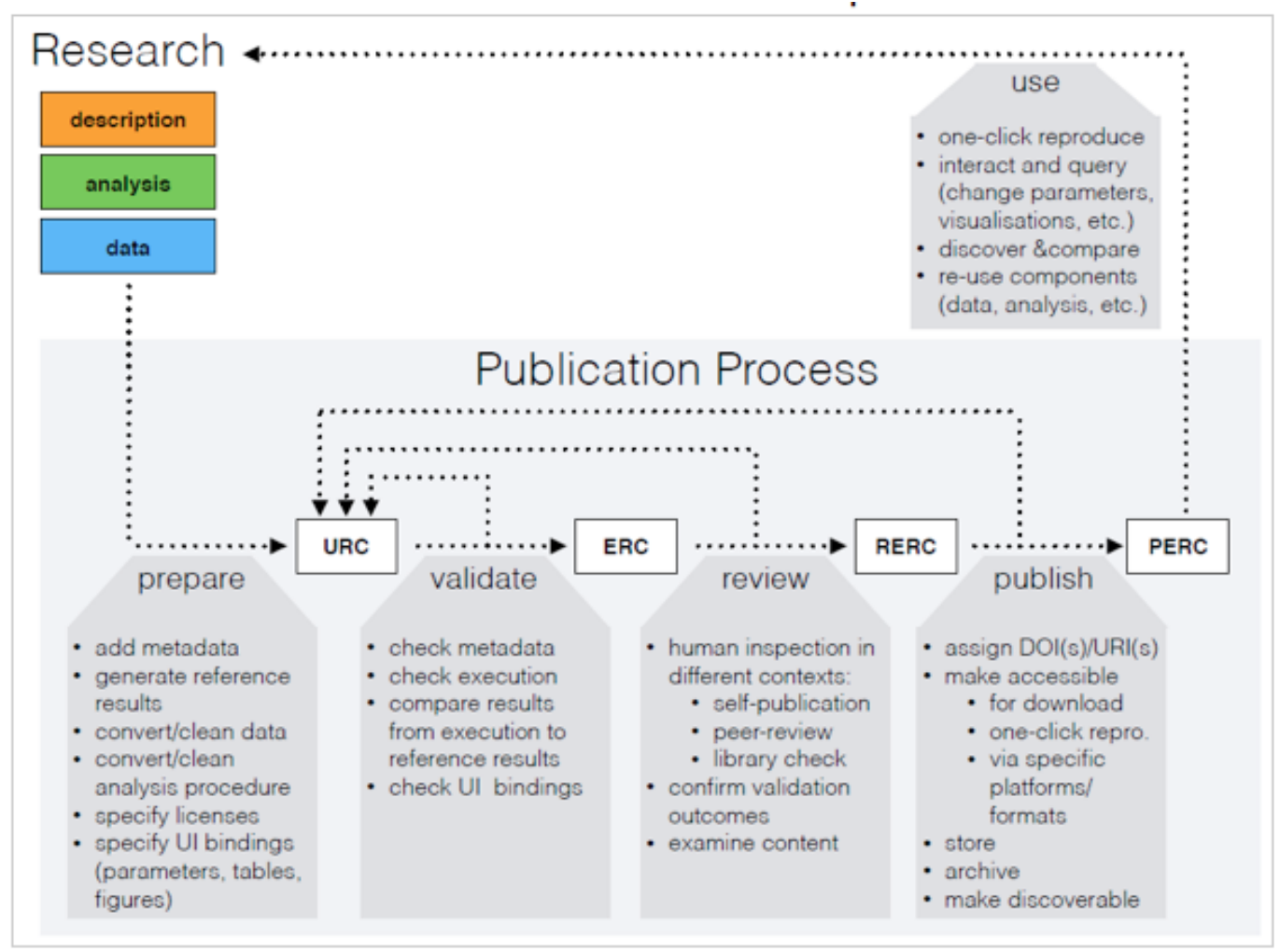

Fig. 4.2: The ERC-based publication process: a research workspace is prepared for a URC, which is validated to become an ERC, which turns into an RERC after peer review, and eventually published as a PERC to be used, e.g. for subsequent cycles.

publish. During this sub-process, a RERC is enriched (e.g. by assigning DOIs/URIs) and then made accessible as a published executable research compendium (PERC). Researchers can reproduce the results reported in a PERC via a single click. They can also reuse components of a PERC (e.g. its data) for their research. In order to make PERCs discoverable and comparable, there has to be a minimum of meta information attached to them.

Finally, if the authors provide UI bindings during the publication process, readers can interact with an ERC in a much broader way. For example, if the authors specify which variables in the code contain specific threshold values, then readers of the PERC can interactively change them - i.e. via an automatically generated UI to explore whether the reported outcomes still hold for different threshold values. Another benefit relates to diagrams and visualizations: readers could select from different visualizations to more easily compare it to a diagram in a second paper.

\subsubsection{Findability and Preservation}

ERCs can have layers of meta information which are divided into reproducibility metadata and discovery metadata. Packages, libraries, and specific versions of 
software is crucial information for reproducibility. Therefore these must be made available for the URC's validation.

The ERC itself is conceptualized as a ready-for-ingest digital asset as defined in the OAIS reference model (CCSDS, 2012). Its outer container uses the BagIt file packaging format by Boyko et al. (2011). It provides minimal metadata and checksums for a file-based payload. The payload comprises at least (i) the Docker (an inner container) and Dockerfile image mentioned above, (ii) metadata files in different formats such as Codemeta ${ }^{32}$, DataCite (Brase, 2009), (iii) the workspace of the conducted research including code and data files, and (iv) created output documents, e.g. a PDF of the original paper. Research data and code can be stored in the image to make distributed reproduction easier but making them accessible as part of the outer container is advantageous for long-term preservation. This is also the case for the metadata.

By design, ERCs encapsulate any information relevant to the reproduction of its contents (cf. "Core parts of ERC"). External contextualization of an ERC can be achieved by generating additional discovery metadata and ultimately connecting the ERC as a whole to the Linked Open Data cloud (Bizer et al., 2009) by using disciplinespecific vocabularies such as GeoSPARQL ${ }^{33}$. This is done to support findability of the ERC. Semantic references make ERC metadata interoperable and ready for discovery.

For the retrieval of software metadata, it is necessary to identify software dependencies. The metadata included in an ERC also provides structural information needed to connect the entry point for execution with the internal structure of the underlying parts (code, data, text, UI bindings). It is, therefore, reasonable to start connecting the available information about the underlying parts as early in the workflow as possible and thus contextualize the files within an ERC.

Our specification also considers preservation requirements by extending bitstream preservation. The re-creation of the original computational environment is vital when re-running the code of a paper. Jon Claerbout's idea of an article being mere advertising of the underlying scholarship has been concisely paraphrased by Buckheit and Donoho (1995, p. 5): "The actual scholarship is the complete software development environment and the complete set of instructions which generated the figures". Consequently, when it comes to preservation, this complete environment must be included and the instructions must be executable. This refers to the internal structure and dependencies.

\footnotetext{
${ }^{32}$ Codemeta: https://github.com/codemeta/codemeta, last access for this and the following URL: 01.04.2019.

${ }^{33}$ GeoSPARQL: http: //www . opengeospatial .org/standards/geosparql
} 
In addition, ERCs can be integrated into the existing ecosystem of persistent identifiers using mechanisms to uniquely and permanently identify the involved resources and agents. Furthermore, metadata comprises explicit license information, ideally using well-known abbreviations or vocabularies, for each component of a compendium. Authors must be assisted in defining data, software, and text licenses, as this is a tedious but crucial aspect of openness - only a proper license allows and defines possible reuse.

The user interface definitions (UI bindings) are an optional element of an ERC and allow for interaction with the included analysis. On top of that, they document the analysis code by providing a guide to the main functions and their manipulable parameters. We see different approaches to generate them ranging from fully automatic to manual definition. Fully automatic UI bindings analyze the code and generate UI widgets based on the input type (e.g. text, number). UI bindings are created by the author, for example, by adding statements in the source code or by using software designed for this purpose.

\subsubsection{Interaction, Exploration, and Reuse}

An ERC not only enables third parties to reproduce the original research results (figures, tables) but also facilitates interaction with them. Because an ERC transparently abstracts various analyses, i.e. it is a "white box", it seems trivial to build a web service which downloads a research compendium from a repository, unpacks it, executes it, and returns the results created. This extends the minimalistic control functionalities described before with relevant features. Once reproducibility becomes easy to use via ERCs, scientists can benefit from the ability to drill deeper into the computational instructions. For example, they can interactively investigate whether the originally reported results change if they manipulate an underlying parameter, which is fixed in a "classical" article. In an ERC they can be exposed as changeable variables, e.g. via dedicated components in the user interfaces such as a slider. The original author could provide information about what constitutes a reasonable range of values for this variable. In addition, being able to inspect analysis procedures more closely can help in detecting errors and in safeguarding the integrity of the publication process. For example, a reader might update an underlying library with known bugs but leave the ERC otherwise unchanged. The recent Reinhart and Rogoff case (Reinhart and Rogoff, 2010; Herndon et al., 2014) showed how damaging it can be when a paper insufficiently describes the analysis on which its conclusions are based. 


\subsection{Discussion}

\subsubsection{Approach}

In this paper, we propose the executable research compendium as a new form for supporting the creation and provision of research results. The four parts of an ERC open up archival of and interaction with computational research with the following improvements: (i) reviewers obtain tools for efficiently validating results in scientific publications submitted by researchers, (ii) results are well-grounded since the research steps and data described in an article are shared, and (iii) other researchers benefit because they obtain tools for accessing, reusing, and extending research components.

The problem of scientists each having an individual workflow is tackled by packaging not just the workspace with data and code, but also the actual publication, the runtime environment, and UI bindings. ERCs not only enable third parties to reproduce the original research and hence re-create the original research results (figures, tables), but also facilitate interaction and recombination, e.g. with other data or new methods. This recombination is complicated and probably feasible only for compatible data respectively computational methods and thus restricted to particular domains.

\subsubsection{Distinction of ERC}

ERCs rely on the concept introduced as research compendium (RC) by Gentleman and Lang (2007). The core difference to an RC is that an ERC is aware of its complete software environment and contains the so-called "transformation software". The "transformation software" generates different views (e.g. PDF documents or graphics) from the RC by processing code chunks within dynamic documents, e.g. by passing them to an interpreter of the so-called "definition language" (Gentleman and Lang, 2007). The authors mention the possibility to include "general purpose software" as part of the "auxiliary software" into an RC in case a version must be specific or might disappear. ERCs take this one step further and embed all software required to run the compendium's code, including the interpreter of the "definition language". It effectively removes all requirements towards the host machine except a Docker runtime.

Research Objects focus on linked data technology. As such, they are characterized by aggregation and referencing of distributed resources, including workflows and their execution. In contrast, ERCs seek to provide consistent packaging for simple reproduction ("one-click") and interlinking within the contained parts. Both ROs and 
ERCs are containers for research data and code needed to preserve and reproduce an analysis, but they approach this goal from different directions (cf. RO bundle ${ }^{34}$ ). ROs focus on the outer perspective: provenance, dependency, interconnections. ERCs target the inner scope: consistency, completeness, independence.

\subsubsection{Creation and Usage}

The two creation patterns support the majority of user workflows. Post-hoc is less intrusive during research, while a template can put good reproducible research practices (e.g. literate programming) into effect. It remains to be examined which approach requires less effort or finds higher uptake by researchers, and if the expected benefits of publishing an ERC instead of a classic paper outweigh the additional efforts for authors. In any case, researchers have to adjust their workflows to remove all "manual" tasks in favor of replicable scripts.

The packaging format specified by the ERC assists different applications (e.g. oneclick reproduce, long-term archival, research information systems) and thus allows future usage independent from the described purposes. The runtime packaging feature is based on open source software with high uptake in industry, but although an open specification process is underway (cf. the Open Container Initiative ${ }^{35}$ ), longevity issues cannot be put aside at this point.

Storing the image and its recipe, i.e. the Dockerfile, increases the chances of reproducing work long after the original publication. With this burden on the Dockerfile, it must be evaluated what criteria it shall comply with for long-term archival because clarity and extensiveness (e.g. explicit versions) outweigh typical concerns (e.g. image size, up-to-dateness). Nevertheless, ERCs mitigate some of Howe's (2012) challenges for virtualization, e.g. reuse and limitations to interactivity.

ERCs do not cover privacy issues, requiring to anonymize data before publication. This restriction holds for other publication forms as well. ERCs cover security concerns because the Docker container provides an effective sandboxing mechanism.

Interactivity is confined by the computation time. Because manipulation of one parameter requires re-running the whole container, an execution platform needs to be transparent and reliable in communicating this issue to the user, e.g. by indicating the expected run duration. The efforts needed to create an ERC can be minimized by using automated metadata derivation. This aspect also applies to the generation

\footnotetext{
${ }^{34}$ RO bundle: https://researchobject.github.io/specifications/bundle/, last access for this and the following URL: 01.04.2019.

${ }^{35}$ Open containers: https : //www . opencontainers . org/
} 
of UI bindings, which must be possible without too much effort by the author. We still have to evaluate to which extent UI bindings can be generated automatically.

\subsubsection{Challenges}

The key challenges for a publishing process based on ERCs are (i) the creation of ERCs must be easy for authors, (ii) ERC-based interaction, discovery, exploration, and reuse must provide sufficient benefits for scientists to result in a broad uptake of the concept, and (iii) ERCs must handle diverse workspaces and integrate requirements from all stakeholders. The adoption of the ERC will be limited if tackling these challenges leads to a system that is too complex or not understood by users.

Some core aspects of the publication process cannot be defined to the required level of detail at this point, namely management of review state (Put review metadata and state into the container or keep it outside?) or transfer and storage (Can journal platforms handle ERC file sizes and execution?). It is inevitable to accompany the concepts in this work by a practical implementation to settle these questions.

Research compendia are designed to support science during preparation, implementation, and publication. However, the definition of ERCs alone cannot enforce correct methodology or proper reviews. Communities of practice have to develop conventions and to expand education to put compendia into effect. Currently, researchers use libraries rarely to curate their work. ERCs can connect the research and library communities as a step towards better digital curation, one of the major challenges for memory institutions of the future.

\subsection{Conclusion}

Reproducible research is a goal with extraordinary meaning for scientific publications. ERCs provide innovation for the publication process by opening its result for reuse. Subsequently, they help to implement the goals of "open science". In this paper, we provide the following key contributions:

- a compendium-based publication process

- reproduction of the computational steps, the results, and visualizations in ERCs

- packaging computational research for long-term reproducibility

- new ways of interaction with research 
Reproducible research can only be realized by creating technical and communicational solutions for the difficulties outlined above. Our design focuses on the interaction of the different roles within scientific research and publication culture. The executable research compendium reduces efforts on the technical side of reproducibility and thus fosters the community's acceptance of openness and reproducibility and creates the basis for open collaborations between scientists.

\subsection{Future Work}

Because ERCs only work when taking care of the specifics of both a scientific domain and the software, we expect a focused solution to deliver the best results for users. We are currently in the process of developing an open, formal specification for ERCs and an open source web-platform allowing users to build, store, execute, and interact with ERCs in the context of computational geosciences in $R$, going beyond most current research data platforms (see Opening Reproducible Research ${ }^{36}$ project on GitHub).

The prototypical implementation will be subject to a series of usability evaluations considering the views of all stakeholders and roles. In particular, usability while creating ERCs will be a crucial factor for the acceptance of ERCs as a form of publication. Although ERCs are designed with existing platforms and workflows in mind, practical evaluation of their successful integration is needed, i.e. a demonstration of a complete publishing process from submission on a journal platform, evaluation during peer review, storage in repositories, publication and interaction on an online platform, and long-term archival. Inherently manual steps of a publishing workflow, e.g. copy-editing, create new challenges for systematic interpretation of the output. A user-friendly interactive execution of compendia has to address open questions regarding parameter transfer and partial container execution.

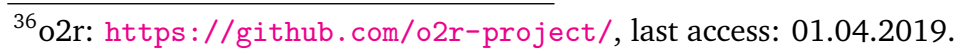





\title{
In-depth examination of spatiotemporal figures in open reproducible research
}

\author{
This chapter was published as: Konkol, Markus and Christian Kray (2018). "In- \\ depth examination of spatiotemporal figures in open reproducible research". In \\ Cartography and Geographic Information Science, 1-16. eprint: https ://doi .org/ \\ 10.1080/15230406.2018.1512421. It corresponds to P4 in the list of publications.
}

\begin{abstract}
Figures such as maps and time series are essential means to visualize spatiotemporal results in scientific papers. Being able to re-compute them using the underlying source code and data is thus a core aspect in reproducible research. However, many scientists see the preparation of code and data for publication as an additional burden without immediate benefits. In this work, we investigate the advantages and new capabilities of reproducible research papers. Our key contributions are (i) the extension of a geoscientist's workflow while examining papers including reproducible figures such as maps and (ii) the prototypical implementation of the workflow as a web application. The workflow is based on current practices of geoscientists and encapsulates benefits of reproducible figures. It is informed by ideas and needs identified by geoscientists in a survey, interviews, and a focus group. Based on their statements, we first extend the traditional workflow steps Discovery and Inspection by additional capabilities and propose two new steps: Manipulation of the content of a spatiotemporal figure and Substitution of the underlying code and data. The extended workflow and its implementation might facilitate in-depth examination and reusability of geoscientific results.
\end{abstract}

\subsection{Introduction}

The main goal of scientists is to create and disseminate new domain knowledge by writing research articles. Explaining this knowledge solely with textual descriptions is hardly achievable concisely and understandably. Hence, figures are popular means to convey new research results and support the reader's understanding. These figures are mostly published in static PDF files, allowing only to look at them but 
not to understand how the results were achieved. This is particularly important for geoscientific papers which often include spatiotemporal results, e.g. spatial interpolations and temperature developments. Maps and time series are popular means to visualize such outcomes (Giraud and Lambert, 2017). Frequently, these figures are produced by source code from data and are consequently a key aspect in reproducible research. We consider research results to be reproducible if all research components underlying the scientific results are publicly available in addition to the paper. Moreover, others can re-run the computational steps by using the same source code, dataset, and configuration leading to the same figures, tables, and numbers which are reported in the paper (Stodden et al., 2016). This form of publishing research holds the potential to address the shortcomings mentioned above. However, publishing code and data is a frequently mentioned burden (Savage and Vickers, 2009). While being able to reproduce results is a definite advantage, it is less clear what further immediate benefits reproducible figures have for geoscientists. In this work, we identify these benefits and show how they can be embedded into the current workflow of scientists working with research articles.

Even if the computations underlying a figure are accessible, manipulating parameters or substituting datasets to see how the results change are time-consuming tasks. In order to solve this issue, reproducible figures could be used, e.g. to create interactive maps which are usually provided by map services but rarely available in papers. By attaching user interface (UI) widgets (e.g. sliders), readers can change the model used for the interpolation map. Such controls might improve a reader's understanding of the analysis and results (Wacharamanotham et al., 2015).

This paper reports on a series of studies investigating how geoscientists currently work with scientific publications and what their needs are regarding reproducible papers. Our key contributions are (i) the extension of their current workflow to reap further benefits from reproducible results such as figures, (ii) and the implementation of the workflow as a prototypical web application.

Our work focuses on publications reporting on computational analyses of quantitative data in the geosciences and on figures showing computational results. Hence, we did not consider qualitative data (e.g. interviews) or figures showing photographs or processes. Consequently, parts of this work might be applicable to other domains although we illustrate our workflow by geo-specific scenarios. In the following, we review related literature. Then we report on the methodology and the conducted studies. Finally, we describe and discuss the extended workflow and its implementation. We conclude by summarizing key insights and briefly outlining future work directions. 


\subsection{Related Work}

First, we briefly review the typical steps of researchers working with scientific papers as a basis for the extended workflow. To put reproducible figures into a broader context, we then review reproducibility in general. The chapter concludes by summarizing work on the role of (interactive) figures in papers and reproducible research.

\subsubsection{Working with scientific publications}

There are two essential steps in a researcher's workflow when examining scientific publications: The Discovery (Monge and Elkan, 1996) phase relates to searching for papers relevant for the own work, e.g. those describing suitable methodologies or study outcomes. Papers can be found using various strategies (Conn et al., 2004) and tools (Renear and Palmer, 2009; Tenopir et al., 2009). Search engines are particularly popular but suffer from several limitations (Wu et al., 2012). Constraining a search query to article components (e.g. figures) can help to address some of them (Bishop, 1999; Sandusky and Tenopir, 2008) as can faceted search (Hearst, 2006; Kules and Shneiderman, 2008).

Several websites provide suggestions (Pain, 2016; Rodriguez, 2015) for efficient paper Inspection. Matarese (2006) suggests browsing, a method focusing on paper components such as graphics. To assess the relevance of a paper, scientists skim through the article and extract key messages, e.g. by examining visualizations (Bishop, 1999; Renear and Palmer, 2009). Tenopir et al. (2009), identified three depths of reading articles: "extracting the key messages", "with great care", and "just to get an idea". Inspection depends on the purpose, e.g. reusing methodologies/results or peer review (Kircz, 1998, Tenopir et al. 2009). To reuse data or code, scientists need to understand them sufficiently and to assess their reliability (Faniel and Jacobsen, 2010). Pham et al. (2012) list several other purposes in the context of reproducible research such as executing the original computations or manipulating datasets and configurations.

\subsubsection{Reproducible research}

Reproducible research is a key aspect in science but perceived differently amongst researchers (Ostermann and Granell, 2017). Several papers discuss the term and provide new definitions (Goodman et al., 2016) or describe the difference compared to terms such as replicability (Patil et al., 2016), which is achieved by independent experiments leading to consistent results (Leek and Peng, 2015). Many scientific 
results are not reproducible due to missing materials such as code and data (Ioannidis et al., 2009; Merali, 2010). Consequently, the "reproducibility crisis" impaired the credibility of scientific outcomes (Donoho et al., 2009). Reasons for unreproducible papers include a lack of incentives and the fear of being scooped (Gewin, 2016). Authors perceive sharing materials as a burden due to the effort required to clean code and data (Savage and Vickers, 2009). However, counter-arguments are increased transparency (Reichman et al., 2011), a better understanding of the study results (Gil et al., 2016), and increased impact of papers including code and data (Piwowar et al., 2007; Vandewalle, 2012). To support publishing reproducible research, several authors suggest guidelines (cf. Barba, 2016; Nosek et al., 2015; Stodden et al., 2016), e.g. writing source code instead of using ready-to-use tools. Thus, the creation of figures is not hidden in encapsulated software features (so-called "black box") but transparent and understandable. Figures often convey results and are thus essential aspects of reproducible research definitions (cf. Claerbout and Karrenbach, 1992). This is particularly relevant for geoscientific papers which frequently include maps and time series to show spatiotemporal results (Giraud and Lambert, 2017).

\subsubsection{Figures in scientific publications}

Figures are key components in scientific papers as they communicate results efficiently (Cleveland, 1984), facilitate the assessment of relevancy (Sandusky et al., 2007), and help understanding (Rolandi et al., 2011). Mack (2014, p. 4) said: "When presenting results, a good graph is like a good scientific theory - once you see it, everything just makes sense." In the geosciences, maps are frequently used to visualize spatial data. They support authors in communicating a specific message and readers in understanding it (DiBiase et al., 1992). Kwakkel et al. (2014) listed several open source tools, which facilitate the creation and exploration of maps in a scientific context. However, they also pointed out the importance of knowing about the underlying dataset. Lee et al. (2017) identified a correlation between the number of citations and visualizations. Still, static figures might hide details of the results (e.g. outliers) making it difficult to challenge the findings (Weissgerber et al., 2016). Reproducible figures can help to avoid and detect errors, e.g. image duplication (Bik et al., 2016). Other error types such as missing or incomplete captions might be less harmful if underlying components are accessible (Cleveland, 1984). In addition, accessible code and data enable creating interactive figures.

\subsubsection{Interactive figures}

Allowing users to control the content of a figure can improve their understanding

(Wacharamanotham et al., 2015). Moreover, interactive figures might tackle issues 
of static illustrations such as hidden details (Weissgerber et al., 2016). There is some evidence that even fundamental interactions such as zooming increase usability and assist scientists in comparing and matching tasks (Adnan et al., 2016; Perin et al., 2013). When using interactive and in particular animated figures it is important to consider their limitations or potential bias (Tversky et al., 2002). Their evaluation should consider both user preference and performance (Hegarty et al., 2009). DiBiase (1990), DiBiase et al. (1992), and MacEachren and Kraak (1997) discussed interactive maps as a tool for exploring and analyzing scientific data. They suggested model steering as a means for studying environmental processes and animated maps which show spatiotemporal data. These means enable researchers to examine different states of the visualized dataset, which is not feasible in static publications (Kwakkel et al., 2014). While publishing interactive results is already possible, it is done rarely. The application Jupyter (Kluyver et al., 2016) combines text and code in one view allowing readers to re-run the computations. With little programming knowledge, readers can manipulate code lines, re-run the analysis to see differences in the results and incorporate UI widgets, such as a slider. RMarkdown (Baumer and Udwin, 2015) is a similar tool but combines source code written in $R$ (R Core Team, 2018) and markdown, i.e. plain text which can be translated to HTML. Similar to Jupyter, RMarkdown enables readers to change the code and to incorporate interactive figures (Chang et al., 2018). Interactive figures are also automatically created by Statsplorer (Wacharamanotham et al., 2015) which supports students in understanding statistical tests. Adnan et al. (2016) observed that even fundamental interactions, e.g. tooltips, led to users feeling more confident compared to static time series and finding it more comfortable to detect maxima and minima (see also Perin et al., 2013).

To sum up, Discovery and Inspection are essential steps in a researcher's workflow and figures play an essential role in scientific papers. Reproducible research allows re-running the computational results using code/data and thus provides new ways to support these steps by enabling interactive figures.

\subsection{Methodology}

To better understand current practices and needs of geoscientists while examining papers, we collected input from them via an online survey, semi-structured interviews, and a focus group. Based on their responses, we identified additional tasks for Discovery and Inspection, and two new phases: Manipulation and Substitution. The code and the data of all three studies are provided in the supplements.

Online surveys are suitable tools for collecting responses efficiently (Lazar et al., 2017). Our goal was to examine how often geoscientists use which figure type and 
what tasks they would like to perform while searching for and examining a scientific paper. We analyzed the data by using descriptive statistics.

To obtain insights into how geoscientists work with papers, the role of figures in geoscientific papers, and potential tasks during paper examination that go beyond traditional inspection, we conducted semi-structured interviews. We applied the grounded theory approach to analyze the data (Glaser and Strauss, 1967). We scanned the transcripts in a line-by-line manner and captured key statements. These were assigned to codes ("open coding") and then grouped to higher level themes. Finally, we grouped the themes into categories which inform the extended workflow described in this paper.

As a third method, we conducted a focus group to collect insights on the same three topics as examined in the interviews. Focus groups facilitate seamless interactions amongst participants and potentially elicit viewpoints and experiences that are different from those of the interviewees. We applied the same grounded theory approach as for the interview data. Subsequently, we describe used materials, conducted procedures, and results of the three studies.

\subsection{Online survey}

To understand scientists' preferences when working with scientific papers, we conducted a survey (see supplemental material).

\subsubsection{Materials}

The questionnaire started with a definition of open reproducible research. Then, we asked if respondents are authors, readers (or both), and for their research fields. Next, respondents who were authors received a list of figure types and indicated, how often they incorporated them in their last five papers. The list was based on Franzblau and Chung (2012) but extended by maps and time series. Respondents then indicated their degree of agreement with statements starting with "While reading a paper related to my research, I would like to ..." followed by activities that support their work, e.g. "... see the code underlying a figure". The same approach was applied for the next two sections: The first started with "It would be useful for my research, if I could search for and access ..." followed by research components, e.g. "... the used source code". The second section started

with "For my research, I would like to search for publications using ..." followed by the options "spatial", "temporal", and "thematic" properties. Respondents used Likert and frequency scales to answer the questions (Lazar et al., 2017). Finally, 


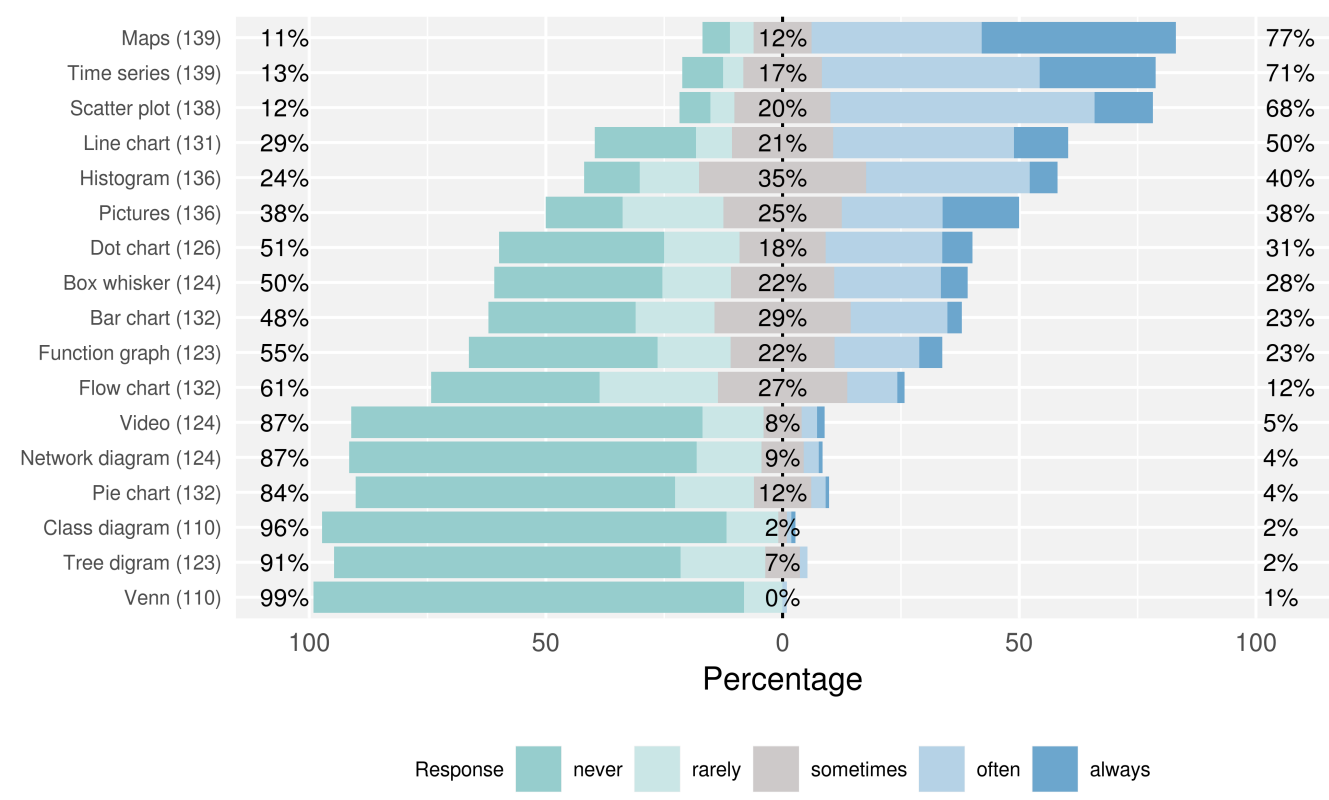

Fig. 5.1: Responses to "Relating to your last five publications, how often did you incorporate the following visualizations?" Numbers in brackets show how many responded to the item. Percentages are grouped into never/rarely, sometimes, and often/always.

respondents had the opportunity to express their thoughts in a comment field. The questionnaire concluded by asking how many years they have worked in research so far.

\subsubsection{Procedure}

We piloted the survey with five Ph.D. students to ensure understandability and incorporated their feedback iteratively. The survey was disseminated via a QR code on a poster that we presented on a geoscientific conference. Moreover, authors who submitted to that conference were contacted via e-mail. 146 geoscientists completed the survey (mean $\mu=17$ years in research, standard deviation $\sigma=9$ years) of which 144 write papers, 140 read papers (139 do both). It was accessible for five months via the open source survey tool LimeSurvey ${ }^{1}$. Survey respondents had to submit the survey actively for it to be included in the analysis.

\subsubsection{Results}

Figure 5.1 shows how often survey respondents included the listed figure types in their last five publications. Maps (77\% incorporated them often or always), time

\footnotetext{
${ }^{1}$ LimeSurvey: https://www. limesurvey .org/de/, last access: 01.04.2019.
} 


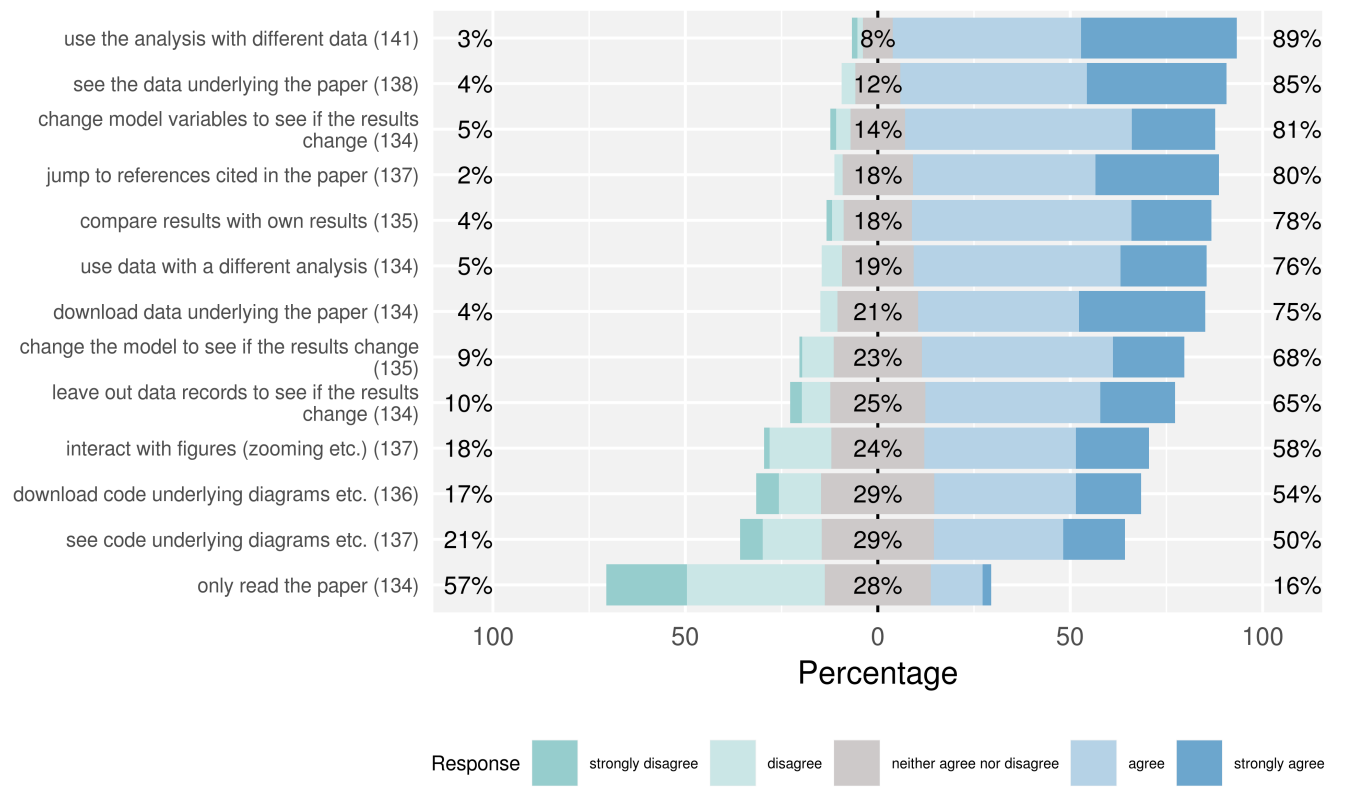

Fig. 5.2: Responses to the question: "While reading a scientific publication related to my own research, I would like to ...". Numbers in brackets show how many responded to the particular item. Percentages are grouped into strongly disagree/disagree, neither agree nor disagree, agree/strongly agree.

series (71\%), and scatter plots (68\%) were used most, followed by line charts (50\%), histograms (40\%), and pictures (38\%). Dot charts (31\%) and box-whisker plots (28\%) were less popular.

Regarding search and access capabilities, respondents preferred methodologies (94\%), (consistent with Bishop (1999)) and datasets (91\%) over code (64\%), research questions (64\%), and hypotheses (63\%). For constraining queries, respondents requested thematic (87\%), temporal (69\%), and spatial (67\%) properties.

Figure 5.2 shows how many respondents requested the tasks while examining a paper. Datasets played an important role. $89 \%$ of the respondents requested to use the analysis method with a different dataset. While $85 \%$ wanted to see underlying datasets, $76 \%$ requested to use it with a different analysis method. Downloading datasets (75\%) and leaving out data records to see how the results change (65\%) were also popular. Tasks related to source code were less popular. Downloading code underlying the computations was requested by $54 \%$ of the respondents. Furthermore, respondents wanted to change the entire model $(68 \%)$ or its parameters $(81 \%)$ to see if the results change. More than half of the respondents requested interactive figures (58\%). Finally, survey respondents requested to compare the reported results with own findings (78\%). The comments did not reveal any new insights concerning the workflow. 


\subsection{Semi-structured interviews}

To receive insights into the current workflow of geoscientists while working with papers, the role of figures in papers, and desirable tasks while examining reproducible research, we conducted semi-structured interviews (see supplemental material). We contacted geoscientists from our faculty personally if they had already published a paper including figures based on computational analyses with quantitative or geographic data such as time series and maps. Nine scientists from geoinformatics, landscape ecology, geochemistry, and planetology (mean $\mu=9$ years in research) were recruited.

\subsubsection{Materials}

The interview had three parts. First, we asked how interviewees work with papers published by other authors. Our questions referred to workflow, goals, difficulties, and emerging questions. We then asked how they search for papers, difficulties during that task, and ideas for improvements. Second, we asked for situations in which figures are (not) helpful. Third, we asked about which static components of papers should be made interactive. Only if interviewees had difficulties in answering this question, they received a sheet with a map and a time series (Figure 5.3) for inspiration. Two interviewees struggled with the question and hence received these figures. By showing two mockups (Figure 5.4), we introduced the idea of a platform that facilitates the reproduction of research results and enables in-depth examination of research components. Then, we asked for criteria and core functionalities which encourage the use of such a platform. The first mockup (Figure 5.4, left) showed the research components, a button for executing the analysis, and the static paper. The second mockup (Figure 5.4, right) showed the interactive view of the paper, i.e. enriched by UI widgets (slider, option buttons) to change the figure content. Afterward, interviewees received a questionnaire for collecting background information.

\subsubsection{Procedure}

We piloted the interview with three Ph.D. students to ensure understandability. We sent the guiding questions to the interviewees one day in advance and interviewed them in their office to ensure they felt comfortable. A consent form informed that participation was voluntary and their statements were audio recorded and treated anonymously. Then we asked the questions and handed out the questionnaire on demographic information. The interviews took 54 minutes on average (duration: 35 

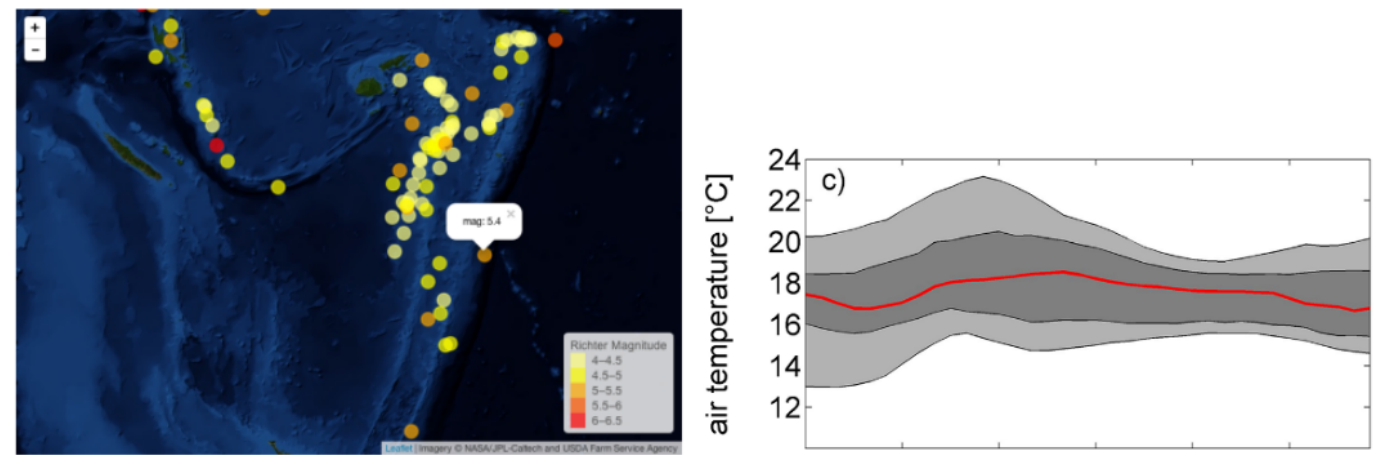

Fig. 5.3: Pictures used to inspire interviewees who struggled with imagining interactive components in static papers. Left: Map of earthquake events in the Pacific (Source: https://cran.r-project.org/web/packages/leafletR/ leafletR.pdf, last access: 01.04.2019) Right: Daytime pattern of air temperature (El-Madany et al., 2016).
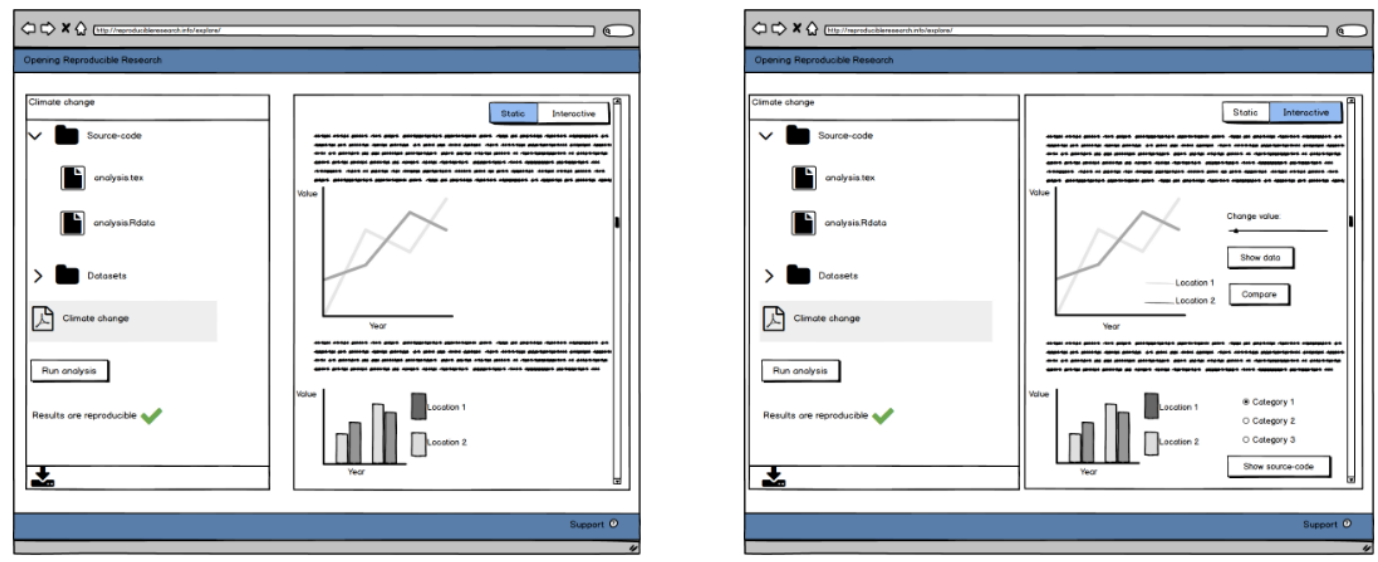

Fig. 5.4: Mockups used to inspire participants and to present the idea of a reproducible research platform. Left: research components, possibility to re-run the analysis in the lower left corner, and the static paper. Right: interactive view enriched by UI widgets to change values. 
to 66 min.). Two interviews were conducted in English, seven in German. Interviews were transcribed literally.

\subsubsection{Results}

Most of the interviewees searched for papers using keyword-based search engines (8 interviewees mentioned that). Three interviewees used suggestions from colleagues or alerts. Two interviewees each looked at citations, into journals, or used internal databases. Further means were logical operators to connect keywords, e.g. AND/OR (2 mentions). The paper search was impeded by difficulties such as finding relevant literature (5), e.g. because search engines did not consider synonyms. Inaccessibility (e.g. paywalls) was another issue (3). For improving search, interviewees suggested constraining queries (3), e.g. by methodology or properties such as chemical substances, using operators (2), and searching for interoperable data (2).

Interviewees had several goals while working with papers. First, interviewees aimed at integrating their research results into the scientific landscape (6). Six interviewees wanted to build upon others' work, e.g. by reusing the methodology. Comparing their findings with existing results (6) and receiving background knowledge (5) were also essential tasks. Three interviewees each aimed at extracting key messages and finding suitable citations. Last, two interviewees wanted to validate their methods or techniques. To achieve these goals, interviewees inspected papers in a superficial and detailed manner. Superficial inspection was about assessing the paper's relevance and extracting key messages. Therefore, interviewees skimmed through the paper and read title, abstract, and conclusion (consistent with Keshav, 2007). Five interviewees focused on figures already during a superficial paper inspection. Only if they could reuse results or acted as reviewers, they continued with a detailed inspection by studying methodology, results, and discussion.

While examining papers, interviewees faced several difficulties. They all struggled with missing details in the methodology section (e.g. regarding configurations) required for a detailed understanding (9). This problem also occurred if research components were missing (3). Missing details in the results section (e.g. missing pvalues) impaired a detailed understanding of the findings (2). Missing visualizations and poorly written abstracts impeded assessing if a paper is relevant (4).

The questions interviewees had while examining a paper addressed methodology (3), e.g. "Why were certain values not measured and compared?", the underlying datasets (3), e.g. "How does the dataset look like and how did they collect it?", 
Tab. 5.1: Requested tasks while examining a paper assigned to the four workflow steps. Numbers in brackets indicate how many mentioned the task (\#interviewees; \#focusGroupParticipants).

\begin{tabular}{|c|c|c|c|}
\hline Discovery & Inspection & Manipulation & Substitution \\
\hline $\begin{array}{c}\text { Constrain search } \\
(3 ; 1)\end{array}$ & See dataset $(7 ; 5)$ & Modify data (5;4) & Switch figure (4;1) \\
\hline Search for data (2;2) & See code (7;2) & $\begin{array}{c}\text { Modify } \\
\text { parameters (5;1) }\end{array}$ & $\begin{array}{c}\text { Substitute dataset } \\
(3 ; 2)\end{array}$ \\
\hline Spatial search (0;3) & $\begin{array}{c}\text { Dynamic figures } \\
(5 ; 1)\end{array}$ & $\begin{array}{c}\text { Re-run with } \\
\text { different software/ } \\
\text { package versions } \\
(0 ; 1)\end{array}$ \\
\hline $\begin{array}{c}\text { Combine keywords } \\
(2 ; 0)\end{array}$ & $\begin{array}{c}3 \mathrm{D} / \text { animations } \\
(5 ; 0)\end{array}$ & & \\
\hline $\begin{array}{c}\text { Executable } \\
\text { supplements }(0 ; 1)\end{array}$ & $\begin{array}{c}\text { Additional } \\
\text { computations }(2 ; 1)\end{array}$ & & \\
\hline Synonyms $(0 ; 1)$ & $\begin{array}{c}\text { Compare } \\
\text { components (2;0) }\end{array}$ & & \\
\hline Similar studies $(0 ; 1)$ & $\begin{array}{c}\text { Re-run analysis } \\
(2 ; 0)\end{array}$ & & \\
\hline
\end{tabular}

and results (2), e.g. "How did they achieve the results and what are the confidence intervals?" Such questions can be particularly relevant during the review process (1).

When inspecting papers, interviewees focused on figures (6) as they visualized key results (4). They helped to understand the paper content efficiently (5) as they showed much information at a glance instead of a sequential textual description. Consequently, readers could better assess if a paper is relevant (2). In addition, figures provided insights into the dataset (5) which was particularly important if it was unavailable. Another benefit was that figures complemented textual descriptions (4) making it unnecessary to read the entire paper. Figures supported readers in accomplishing complex tasks (4) such as recognizing patterns or comparing numbers.

Interviewees also mentioned disadvantages of figures. The message of a figure might be irrelevant (6) resulting in wasted time to understand it. Next, it was unclear how it was computed (5), particularly if code and data were unavailable. Related to that, figures often aggregated numbers thus hiding exact values (3) and impeding comparisons with other figures. Still, figures required descriptions for a better understanding (4). Otherwise, they were more confusing than helpful (2). Next, readers were limited to the figure selected by the author (3) and could not change the visualization. Also, the figures did not show how the authors handled outliers (2). Last, figures were seen as unsuitable for complex visualizations (3), e.g. temporal developments shown in maps. 
We asked interviewees for their ideas to integrate interactive components into static papers and core functionalities of tools and services which support working with reproducible research (Table 5.1). Addressing the limitations of papers and figures mentioned above, inspecting code and data to understand research results was the most popular feature (7). Furthermore, interviewees requested to manipulate datasets (5) and model parameters (5) to see how results change and to test the reported assumptions. Three interviewees suggested substituting the data by another compatible one. Comparing datasets and figures require supporting tools (2), e.g. a side-by-side comparison or an overlay. Finally, two interviewees requested to re-run the computational analysis (2).

"If all datasets are accessible in a certain format [... ], I could reproduce the work and do own investigations without having additional effort, without thinking of data transformations." (Interviewee 4, translated from German)

Next, interviewees requested dynamic figures (5), such as zoomable maps and diagrams showing exact values. To counteract the limitations of specific figures, four interviewees proposed switching the given visualization to a different one. They also proposed three-dimensional visualizations/animations describing the methodology (5) and additional computations (2), e.g. statistical summaries. While examining the interactive paper, readers requested features to notify the author about new findings or to discuss them with other researchers (4). Participants identified a number of potential benefits of these tools: reusing research components (3), deeper understanding (2), supporting reviewers (2), meta-analyses (2), collaboration (1), validation (1), and cross-checking (1).

Authors require tools to realize these features and interactive figures. When asking for key criteria for reproducible research platforms which encourage usage, interviewees' main consideration was the copyright for data and code (5). Tools should not be time-consuming (4) and able to handle the diversity of research components (4).

"I would be happy to provide all required files as long as it (reproducible research platform) can deal with my messy code. So, especially for older papers, it is really a mess. I would have troubles to understand it again." (Interviewee 1, direct quote)

Two interviewees said publishing code is not beneficial as understanding it is too time-consuming. Three interviewees expected features to acknowledge reused work (3). Moreover, two interviewees requested mechanisms to encourage collaboration 
between researchers, e.g. if readers intent to reuse published materials. Hence, acknowledgment results in citations and co-authorship. Trust is another issue. While three interviewees feared misuse of their data, e.g. by commercial enterprises, two interviewees expected trusted providers, e.g. journals. Interviewees feared to be scooped when making research components publicly accessible (4) (consistent with Nosek et al., 2015). Three interviewees acknowledge that the tools facilitate detecting errors but had concerns regarding researchers' reputation. Furthermore, the features could lead to new scientific insights raising the question of when they result in a new paper (1).

\subsection{Focus group}

To complement the insights from the interview and the survey, we organized a focus group. We recruited five additional geoscientists from geoinformatics and landscape ecology using the same criteria as for the interviews (mean $\mu=5$ years in research).

\subsubsection{Procedure}

The focus group had the same three parts as the interview. We learned from the interviews that showing mockups (Figure 5.4) before asking for core functionalities of a reproducible research platform resulted in participants focusing on the pictures. Hence, we showed the mockups afterward and then asked for situations in which they (do not) benefit from it. Participants received the guiding questions one day in advance and the same consent form as the interviewees. Afterward, we briefly introduced the topic, asked participants to introduce themselves, and then asked the questions. Finally, participants received the same questionnaire on demographic information as the interviewees. The focus group took 86 minutes and was conducted in German. The statements were transcribed literally.

\subsubsection{Results}

Many statements of the participants confirm what the interviewees said (Table 5.1). Below, we only report on the differences. Typical challenges while searching for papers were receiving an overview of the search results and specifying queries to reduce the results list. For improving the search, participants suggested constraining the search by spatial properties or those papers having executable code attached. Regarding interaction possibilities and core functionalities, one participant considered that different software versions could lead to deviating results and therefore suggested changing the version when executing the analysis. One participant named 
benefits such as transparency and credibility. Finally, participants developed the idea of a meta-analysis which can be updated regularly with new data. Relating to incentives, participants suggested journal guidelines and solutions to avoid reputation damage if others find errors in the research that the author laid open.

"The way of publishing changes. You are aware others look at it in a different way. Maybe you are more careful which might be an advantage. I think it needs to lower the fear of authors becoming vulnerable." (Participant 1, translated from German)

\subsection{The extended workflow for reproducible research papers}

The three studies revealed insights into the workflow of geoscientists working with papers. Discovery, i.e. the search for papers, and Inspection, i.e. the examination of the content, are two essential steps. Participants requested tasks which are infeasible if research is not reproducible, e.g. re-running the analysis or changing the parameters underlying a figure. While some of the tasks refer to Discovery and Inspection, others exceed current practice. Hence, we describe Manipulation and Substitution to follow the first two phases. Figure 5.5 shows the four steps which comprise the tasks listed in Table 5.1. We also consider their contribution to a reader's understanding. Finally, we identify Comparison as a cross-cutting task relevant within each step.

The workflow step Discovery allows fine-grained search queries compared to a fulltext search. Researchers can create queries including spatial, temporal, and thematic properties which can be extracted from the data and the source code. If code is available, geoscientists can discover papers which include maps based on a specific interpolation functionality (e.g. krigeST from the $R$ package gstat). Consequently, researchers can search for papers more efficiently and gain initial insights into the paper content. This is particularly relevant for geoscientific papers, which frequently include maps and time series based on computational analyses.

When a relevant paper is discovered, geoscientists continue with its Inspection. Readers are not limited anymore to the textual description of the conducted research. They can thoroughly examine the dataset and the code underlying the reported results and figures. Readers might learn how a figure was computed. This functionality can be facilitated by highlighting those code lines and data subsets which underpin a specific figure. Readers can thus understand how a map showing an interpolation was computed and are better able to reuse the underlying code for their 


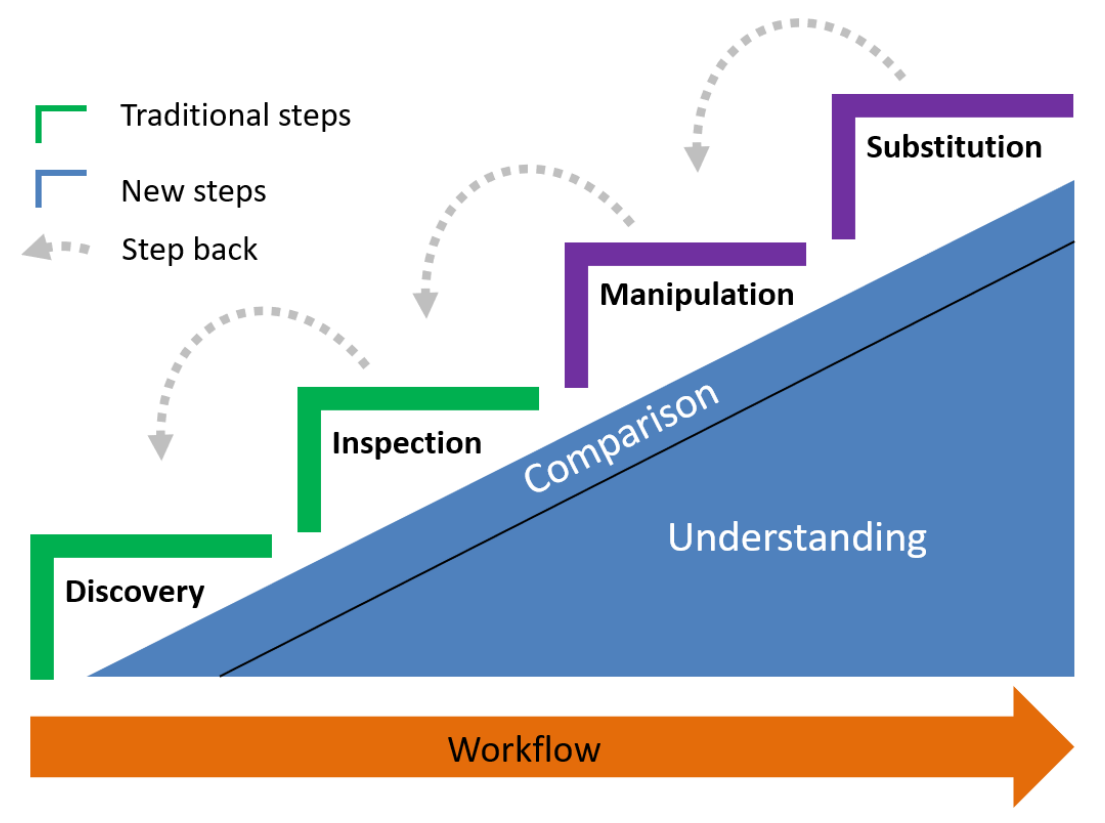

Fig. 5.5: The extended workflow for examining reproducible scientific papers. The steps Discovery, Inspection (passive tasks, e.g. looking at components), Manipulation, and Substitution (active tasks, e.g. modifying parameters or replacing underlying components) each contribute to the reader's understanding. The knowledge gain is probably not equal as suggested by the step height. Comparison is considered as a cross-cutting task relevant in each step.

work. Examining data processing steps and configuration details might counteract missing information in the methodology section. The Inspection phase answers questions raised in our studies, e.g. "How does the dataset look like" and "How did they achieve the results" which is not possible in traditional papers.

After understanding the materials, readers proceed with the Manipulation phase. Here, geoscientists do not only look at the paper and the materials but become active readers. Manipulation comprises all tasks which allow readers to modify what underlies a figure. In the kriging example, readers can change the model of the underlying variogram (e.g. gaussian, spherical, exponential) and manipulate its parameters (sill, range, nugget) to assess the influence on the interpolation uncertainty. Such tasks can be facilitated by UI widgets, e.g. a slider for changing parameters. This phase holds the potential to enhance the understanding of the mechanisms underpinning a figure. Readers might learn how changes in the configuration defined by the author affect the results. They can test assumptions and see if the findings still hold for different settings. Manipulation is not meant to be an isolated step. Readers should be able to modify parameters and inspect materials at the same time. Otherwise, users cannot learn about the internal processes ("black boxes"). 
In the Substitution phase, readers are not restricted to the underlying research components anymore. For example, they can re-run the kriging analysis after replacing the original dataset by their own compatible dataset of a different region and see the new result. Moreover, readers can merge datasets from different publications to replace the original one. Similarly, readers can replace the original algorithm, e.g. to try out a different interpolation implementation. Consequently, readers might learn about the portability of the results or create new scientific insights. Geoscientists might become encouraged to reuse research outcomes for own work resulting in acknowledgment for the original author and less work for the reader. Again, Substitution is not an isolated step but can be combined with manipulating the new results.

Comparison is a cross-cutting task relevant for each step and a reader's understanding. During Discovery, geoscientists might want to compare metadata about the paper or the research components after searching for papers located in a user-defined area or temporal constraint. During Inspection, readers might want to compare, e.g. results and figures, within a paper or between different papers. The same applies to Manipulation. Therefore, the assets should be presented in a way which facilitates readers to identify and understand differences, e.g. in a side-by-side manner, by combining them into another visualization, or by an overlay. It should be possible to compare the original kriging interpolation with a map in which the reader changed the parameters. The same applies to Substitution. Readers should be able to compare figures before/after replacing input datasets. Another issue arises if two different map types should be compared. If code and data are accessible, we might transform two incomparable map types into a third map type which facilitates the comparison task.

\subsection{Realization of the workflow}

We implemented a prototypical web application to realize the extended workflow presented in the previous section (Figures 5.6-5.9). It supports readers during the traditional workflow steps Discovery and Inspection. Furthermore, we implemented tools to assist readers in achieving the two additional steps Manipulation and Substitution.

Therefore, we built upon the concept of an Executable Research Compendium (ERC) (see chapter 4). It encapsulates the runtime environment and all research components, i.e. source code, data, and metadata, required to re-run the analysis using Docker, an open source solution for encapsulating applications in a container. Readers can thus execute and examine the analysis underlying the reported results. We 


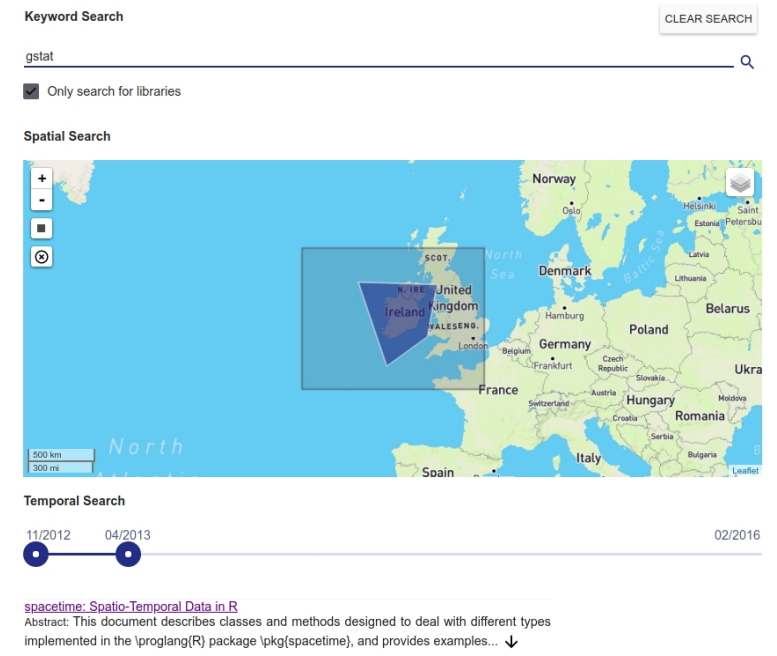

Fig. 5.6: Implementation of the workflow step Discovery. Readers can create queries using spatial (map), temporal, thematic (keywords), and source code-specific properties (libraries).
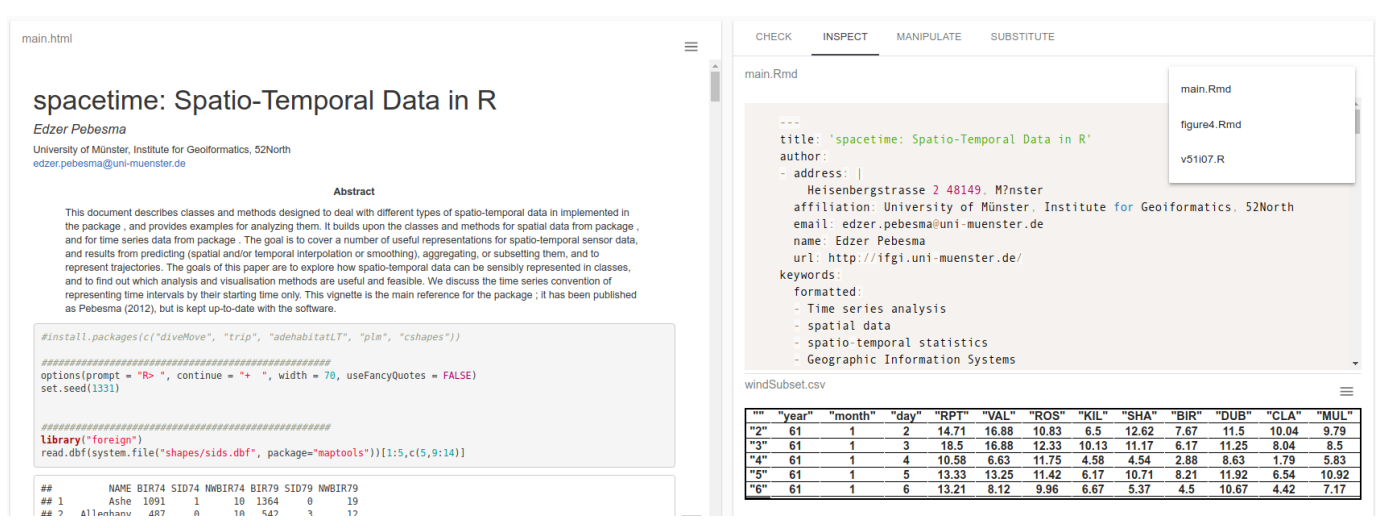

Fig. 5.7: Inspection: Readers can read the paper (left) and at the same time examine the underlying source code and dataset to understand how the reported results were computed (right).

used the services to create and execute ERCs ${ }^{2}$, to extract metadata from the datasets and the $R$ scripts $^{3}$, and the application programming interface (API) ${ }^{4}$. We demonstrate its application using the paper about the spacetime package for $R$ (Pebesma, 2012). Consistent with the scenario mentioned above, the paper has code attached which includes a functionality for kriging interpolations.

To find papers relevant for their research, readers can first search for publications by combining spatial (bounding box), temporal, thematic (keywords), and source code-specific properties, e.g. a certain library used in the $R$ script (Figure 5.6). When

\footnotetext{
2o2r-platform: https://github.com/o2r-project/o2r-platform, last access for this and the following URLs: 01.04.2019.

${ }^{3}$ o2r-meta: https://github.com/o2r-project/o2r-meta

${ }^{4}$ o2r-web-API: https://github.com/o2r-project/o2r-web-api
} 
they find a suitable paper, they can read it as before but at the same examine the $R$ script and the data used to compute the numbers and figures in the paper (Figure 5.7). In the next step, they can change parameters used to produce a specific figure and see how the results change or if the assumptions are robust. In the kriging example, they can manipulate the model underlying the variogram and inspect only those code lines and data subsets used to produce the particular figure (Figure 5.8). Finally, readers can check if the results are applicable to other scenarios, e.g. by substituting the dataset underlying the paper by a compatible one from another paper (Figure 5.9).

To sum up, the workflow steps address the issues mentioned by the study participants. The extended search capabilities support finding relevant papers. Being able to inspect data/code makes research more transparent. By manipulating parameters, readers might better understand them. Substitution might facilitate reusing materials.

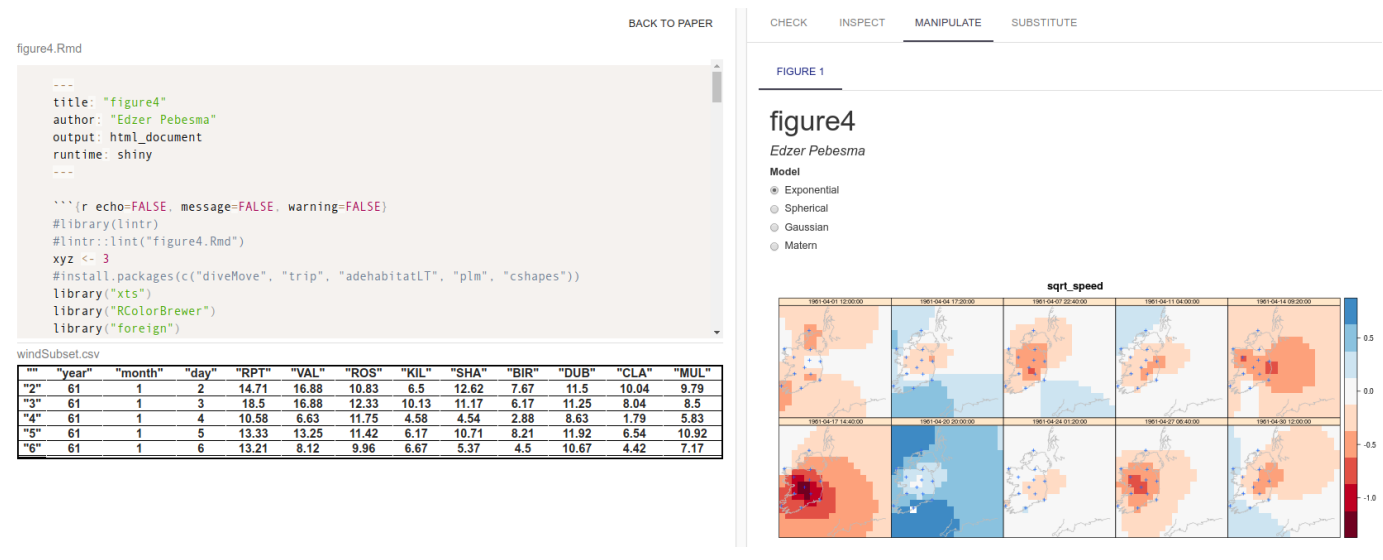

Fig. 5.8: Manipulation: Readers can change the model of the variogram underlying the kriging interpolation by using option buttons to see how the results change (right), and examine only those parts of the source code and data subsests that were used to produce the figure (left).

\subsection{Discussion}

One might argue that the presented workflow is not geo-specific. The extended workflow could be equally relevant, e.g. for biologists or psychologists working with computational statistics. This aspect is not a limitation but indicates the general need for reproducible research which can be examined in detail. However, the workflow steps encapsulate needs mentioned by geoscientists and thus primarily address the geoscientific domain. A typical characteristic of geoscientific papers is the use of maps and time series (see survey results) to show spatiotemporal results based on computational analyses. Although we showcase the approach using maps, the 


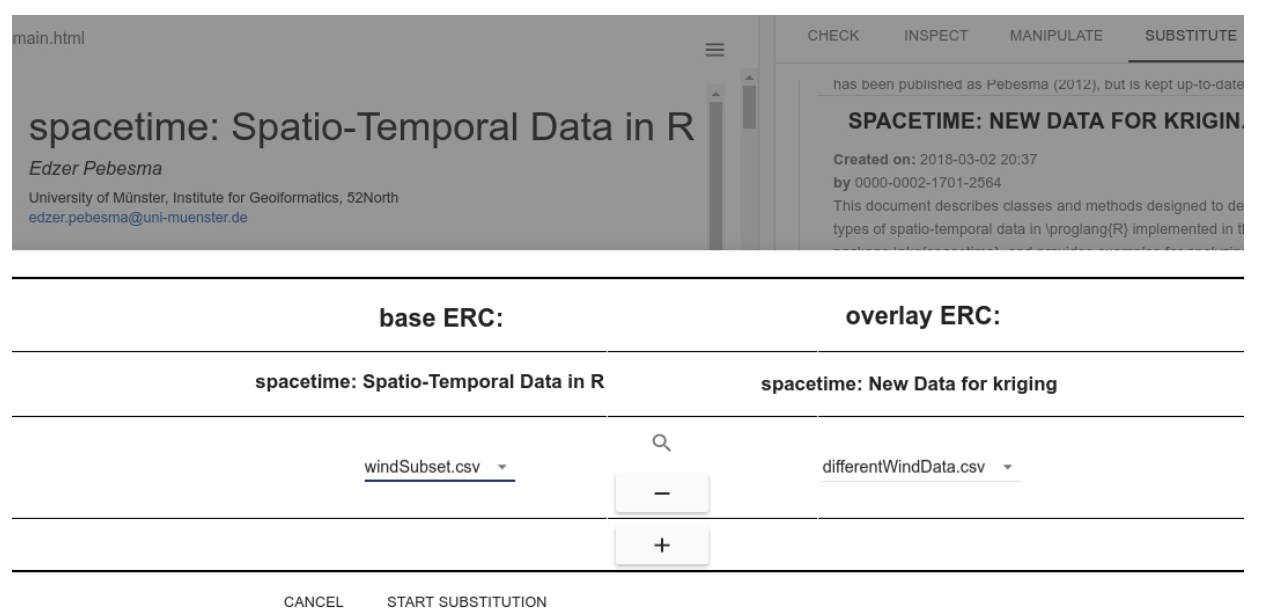

Fig. 5.9: Substitution: Readers can substitute the dataset underlying a paper (base Executable Research Compendium, ERC) by data from another paper containing compatible data (overlay ERC).

workflow can be applied to other figure types produced by code from data. Hence, reproducible figures, i.e. figures which can be re-computed using the same data and code, are particularly important.

The workflow is beneficial for geoscientists who aim at reusing results, e.g. geographical analyses and spatiotemporal data. Reviewers can better assess the assumptions made by the authors and validate the findings prior to their publication in a more efficient way. The two user groups require an understanding which goes beyond extracting key messages. The workflow facilitates this understanding by providing access to all materials used to compute the results. Readers can understand the mechanisms underpinning the figures, and thus assess reusability. Easily manipulable figures can also be part of a superficial paper examination, e.g. to quickly assess the robustness of the results by changing the parameters to see effects on the interpolation uncertainty. Although each workflow step results in additional knowledge about the paper, the individual contribution of each step to a reader's understanding remains open. The knowledge gain after each step is probably not equal as suggested in Figure 5.5. It is also unclear if completing the steps is required and preferred in the proposed order. Readers can start by substituting the original dataset with data from another region. However, they probably can only take full advantage if they understand the original data and code, apart from assessing interoperability.

Understanding how results were computed is not solved by showing data and code alone. Readers still need some programming knowledge and support, e.g. by showing code subsets of a specific figure. Hence, we realized the workflow as a prototypical implementation to assist readers in achieving these tasks. The application provides contextualized access to underlying research components, such as code and data 
used to compute a specific figure or number. It can be used to conduct user studies, e.g. about how the extended workflow affects a reader's understanding. Moreover, we can evaluate if reviewers can assess a paper's correctness and validity more efficiently than by reading the static paper.

The prototypical application provides initial evidence for the overall realizability of the workflow. However, the implementation also reveals open questions and issues. First, participants mentioned they would like to inspect the data underlying a paper. Such support can range from solely displaying the data to complete data exploration packages similar to Tablaeu (Murray, 2013) and QGIS (QGIS Development Team, 2018). More research is required to identify which tool support is needed for data inspection. This task might differ if readers examine a scientific paper compared to the work with geographic information systems. Next, the manipulation of figures requires re-running the code with the new parameter. The computation time of the example paper was short allowing to see the new results immediately. Nevertheless, readers will probably not want to wait for longer computations. Consequently, mechanisms to preprocess the result with possible parameters might be needed or at least a clear indication for how long computations will take. Third, scientists use different data formats (e.g. Shapefile and GeoJSON) to store spatiotemporal data. Consequently, there are two critical challenges in the context of the substitution step. Substituting datasets can only be successful if they are interoperable. Open data, which is not only about accessible data but also about open formats, at least partially tackles this issue. Open formats such as GeoJSON for simple use cases and GML (Geography Markup Language) for more complex scenarios facilitate software to read and handle the data. Second, datasets stored in the same format are not necessarily compatible, e.g. due to different data types or column names. User interfaces similar to text editors might support readers to rename or rearrange columns to make their dataset compatible with the original one. In addition, authors might publish their data as linked data, i.e. semantically enriched, machine-readable, and connected to external datasets (Bizer et al., 2009).

Results: We categorized the responses of the participants into the presented workflow steps. Most of the statements referred to Discovery and Inspection. Fewer statements were related to Manipulation and Substitution. One reason might be that Discovery and Inspection are frequent tasks in a researcher's workflow to which everyone can contribute. In contrast, manipulating figures and substituting datasets is rarely possible and, even if materials are provided, rarely done as supportive interfaces are missing. The survey results show that the listed activities (Figure 5.2) are popular tasks. The lowest value is $50 \%$ (for seeing code) which is still high enough to motivate its implementation. Source code received less attention from the study participants than datasets. Fewer statements on requested features, usage criteria, and incentives related to source code. Seeing and downloading it appears 
to be less important. Code-related tasks in the questionnaire were desired to a lesser extent. One reason might be that study participants had little programming expertise and could thus not reuse code - one interviewee stated coding is not standard in their field. Overall, the statements on figures in publications confirm their essential role in research (Bishop, 1999; Claerbout and Karrenbach, 1992).

Reproducible research is a prerequisite for most of the requested tasks but rarely done in practice. As shown in our implementation, approaches such as Executable Research Compendia (see chapter 4) are suitable concepts to realize the requested tasks. Reproducible research contributes to solving several issues identified by participants. Attaching code and data increases transparency and discloses the computations including all details underpinning the reported figures and results. Readers are thus better able to follow the analysis and reuse data and code. Spatiotemporal analyses are hardly describable with text alone. Hence, figures such as maps showing results are essential means in the geoscientific domain (Giraud and Lambert, 2017). Being able to re-compute them is a crucial aspect of reproducible research. Including openness using open source tools and non-proprietary data formats can help to avoid compatibility issues. Another vital issue, the reluctance of sharing materials, cannot be solved by the workflow and its implementation alone. It is essential to have a discourse within the scientific community and a shift towards "open science" (cf. Markowetz, 2015). The workflow and the implementation are supportive tools that reveal further benefits of open reproducible research. As a result, these benefits might encourage authors to share their data and source code.

Limitations: We first asked interviewees for interaction possibilities, then showed mockups for supporting tools and afterward asked for core functionalities. It seemed that participants focused on mockups too much. This confirmed the provided features but asking the question on core functionalities before and after the mockups, which we did in the focus group discussion, might have resulted in less biased statements. Nonetheless, the answers on the preceding question about interaction possibilities were similar to the statements on core functionalities which might have counteracted this issue.

\subsection{Conclusion}

Publishing reproducible research is perceived as being good scientific practice. However, enabling others to re-run the analysis does not incentivize authors to invest time into the preparation of data and code. In this work, we presented additional benefits geoscientists gain if results, particularly figures become reproducible. We focused on figures such as maps which are popular means to communicate spatiotemporal results in geoscientific papers. The new capabilities were derived from a series of 
studies with geoscientists. Our key contributions are an extended workflow for examining reproducible publications and the prototypical implementation of the workflow as a web application. Using the example of a map showing an interpolation, we described additional capabilities during the Discovery and Inspection phase and presented two new steps to follow these two phases. Manipulation comprises tasks which allow readers to modify settings underlying a figure, e.g. by changing a model parameter. Substitution refers to replacing research components, e.g. the dataset or the algorithm by alternative, compatible ones. The Comparison of results, e.g. the original map and the manipulated one, was considered as a cross-cutting task relevant within each step. The workflow steps encapsulate benefits for readers: Discovery provides spatiotemporal and code-related information and thus initial insights into the analysis which is useful to assess the paper's relevance. During Inspection, readers can understand the materials underlying the results, e.g. the geostatistical analysis used to compute the map. Manipulation facilitates reviewers to test assumptions and validate the findings. Finally, Substitution enhances understanding of the applicability of the results, e.g. by using a dataset from another region or a different interpolation algorithm.

The presented workflow particularly supports a detailed paper examination, to test assumptions, or build upon results. Hence, it would be interesting to conduct further studies which focus on the needs of reviewers and those who reuse research components. We will pay particular attention to how readers interact with the workflow steps and to what extent the steps contribute to readers' understanding. The studies in this work revealed issues which were not central to this paper but require further research, e.g. how to handle copyright issues for paper, data, and code. We will also design supportive tools for authors to create interactive figures. These tools collect information about which data subsets (e.g. a specific column) underlie a figure thus simplifying the substitution with other datasets. We will integrate these tools and the extended workflow into existing frameworks such as Open Journal Systems ${ }^{5}$.

\section{Supplemental material}

A reproducibility package including materials for the survey, interviews, and the focus group is accessible as an Open Science Framework project ${ }^{6}$. The code for the web application can be found on GitHub ${ }^{7}$.

\footnotetext{
${ }^{5}$ Open Journal Systems: https://openjournalsystems.com/, last access for this and the following URLs: 01.04.2019.

${ }^{6}$ Supplemental material: https://osf .io/brjtg/?view_only=1354063fe3c34a95954dd5ecec85f583

${ }^{7}$ o2r-platform: https://github.com/o2r-project/o2r-platform
} 


\section{Acknowledgments}

We are grateful to Max Pfeiffer for proofreading and inspiring discussions. 


\title{
Creating interactive scientific publications using bindings
}

\author{
This chapter is under review: Konkol, Markus, Christian Kray, Jan Suleiman (2018). \\ "Creating interactive scientific publications using bindings". Proceedings of the ACM \\ on Human Computer Interaction. https://doi.org/10.31223/osf.io/jtd8c. It \\ corresponds to the P5 in the list of publications.
}

\begin{abstract}
Many scientific publications report on computational results based on code and data, but even when code and data are published, the main text is usually provided in a separate, traditional format such as PDF. Since code, data, and text are not linked on a deep level, it is difficult for readers and reviewers to understand and retrace how the authors achieved a specific result that is reported in the main text, e.g. a figure, table, or number. In addition, to make use of new the opportunities afforded by data and code availability, such as re-running analyses with changed parameters, considerable effort is required. In order to overcome this issue and to enable more interactive publications that support scientists in more deeply exploring the reported results, we present the concept, implementation, and initial evaluation of bindings. A binding describes which data subsets, code lines, and parameters produce a specific result that is reported in the main text (e.g. as a figure, table, or number). Based on a prototypical implementation of these bindings, we propose a toolkit for authors to easily create interactive figures by connecting specific UI widgets (e.g. a slider) to parameters. In addition to inspecting code and data, readers can then manipulate the parameter and see how the results change. We evaluated the approach by applying it to a set of existing articles. The results provide initial evidence that the concept is feasible and applicable to many papers with moderate effort.
\end{abstract}

\subsection{Introduction}

Many scientific articles include computational results, such as figures, tables, and numbers, that are based on source code and data. For this reason, the "open science" initiatives "open code" and "open data" request authors to publish the materials 
underlying these results. Readers and reviewers should be able to understand how the authors produced the computational results, which parameters were used for the analysis, and how manipulations to these parameters affect the results. Reviewers and readers could thus check whether the results vary after changing the original parameter set and reuse the materials for their own research. Increasingly, journals and funding agencies are instituting guidelines to ensure that researchers share their materials.

However, the way research is published has not yet adapted to these advancements. Scientific insights are still published as static texts, i.e. in PDF documents or HTML renderings. These published papers only provide links to supplemental materials and are thus largely disconnected from the underlying code and data. Consequently, gaining a deeper understanding of the analysis presented in the paper requires extensive effort, including downloading the supplements, opening the code file in the local environment (if installed), and searching for the right source code lines and data subsets. In addition, it is challenging to find out which parameters underlie a statistical analysis and how changes to their initial configuration affect the final output. Hence, readers might not even try to obtain a detailed understanding of the code used to produce the results. One way to simplify the inspection of the underlying materials and the manipulation of parameters would be to create interactive papers with the help of user interface (UI) widgets. UI widgets, such as a slider or radio buttons, are interactive elements which provide readers with some control over what is shown, for example, in an interactive figure (Rathfux et al., 2016). However, such figures are rarely part of articles, as creating them is time-consuming.

The key contributions of this work are threefold: First, we present the concept of bindings in the context of scientific publications. The goal of a binding is to assist readers and reviewers in inspecting the code and the data underlying a specific result in the paper, in manipulating the underlying parameters to see how the output changes, and in substituting the dataset underlying the analysis. In order to achieve those goals, a binding describes which source code lines and data subsets were used to produce an individual computational result, such as a figure, table, or number in the text. A binding can then be used to create an interactive figure by connecting a UI widget to a parameter. Second, we describe an initial prototypical web application for authors to create a binding, and for readers to use the resulting interactive figure. The system assists authors in selecting the code lines, specifying the parameter, and configuring the UI widget. Based on the resulting binding, the system creates an interactive figure for readers, who can then use it in parallel to reading the text. Third, we showcase the feasibility of a binding by applying it to 83 reproducible figures in 20 existing scientific publications. The results show that bindings are applicable to scientific articles because many of the figures in the papers 
are produced by computational analyses including parameters. We successfully created 83 interactive figures using a slider or radio buttons which manipulate the initial configuration of the parameters and thus the final output. We will also provide an initial assessment of the effort required to create a binding and the time needed to use the interactive figure.

Scope: The presented approach addresses papers which include computational results in the form of statistics or analyses. We thus do not consider papers describing conceptual results, frameworks, information systems, photos, or diagrams based on ready-to-use software. Due to the focus on statistics and to keep the scope of the work manageable, we only consider computational results based on $R$, a programming language for statistics (R Core Team, 2018). Bindings require reproducible research results, which means that we can use the same data and code to achieve the same results as reported in the original paper (Goodman et al., 2016).

In the remainder of this article, we first review related work and then present the concept of a binding followed by its prototypical implementation. Afterward, we describe the evaluation and discuss the results. Finally, we conclude by summarizing key insights and future work directions.

\subsection{Related Work}

In the following sections, we review work on "open science" practices, how research materials can be connected to increase transparency, and possibilities for creating interactive papers.

\subsubsection{Publishing research materials}

The publication of research materials, such as source code and data, is a key aspect in "open science". "Open science" summarizes initiatives that aim at improving the accessibility, reproducibility, replicability, and understandability of the scientific results reported in an article. These initiatives address individual aspects, for example, publishing "open access" articles (Harnad et al., 2004), releasing "open data" (Gewin, 2016), providing access to "open code" (Easterbrook, 2014), and using "open source software" (Steiniger and Hay, 2009). Still, for a number of reasons (e.g. effort), authors are reluctant to publish all research materials (Barnes, 2010). Several studies from different domains have checked the accessibility of materials underlying certain computations and had poor results, for example, because code and data were missing (see, chapter 2 as an example in GIScience and Echtler and Häußler (2018) in HCI). Several tools and concepts exist which try to minimize the effort authors 
have to put forward to publish all their materials, e.g. source code and data, in an open way. A fundamental concept is the research compendium (Gentleman and Lang, 2007), which is essentially a project folder containing all materials that are needed to re-compute the scientific results. Online applications, such as Open Science Framework $^{1}$ provide the infrastructure to realize this form of publishing research. In addition, the OntoSoft ontology describes metadata for research software in order to facilitate the reuse and sharing of existing software (Gil et al., 2016). Nevertheless, access to these materials does not guarantee reproducibility of the computational results, e.g. the figures, tables, and numbers (Stodden et al., 2018). Technical issues might impede code execution, and the reproduced figures might be different from the original ones (see chapter 3). For this reason, several other approaches focus on executable environments: A Sciunit, for example, is a reusable research object allowing re-computation of the analysis in a container (That et al., 2017) (see also ReproZip by Chirigati et al. (2016)). A similar approach is the Executable Research Compendium (ERC) (see chapter 4) which also considers the publication workflow of a scientific article. In order to demonstrate the potential of ERCs, chapter 5 suggested and implemented an extended workflow for readers while examining a scientific article that was submitted as an ERC. The workflow contains the steps "discovery", i.e. finding relevant literature based on information included in the source code (e.g. a library or a function), "inspection", i.e. in-depth examination of code and data underlying the computational results, "manipulation", i.e. changing the parameters used in the analysis, and "substitution", i.e. replacing the used dataset with another compatible one. The realization of these steps requires more than just accessible and reproducible source code, but it also requires fine-grained connections between text, data, and code down to the level of parameters.

\subsubsection{Connecting paper, code, and data}

The approaches presented above strongly focus on accessibility and reproducibility. However, the paper, the code, and the data are still disconnected, making it difficult for readers to relate the results reported in the paper to the source code and the data subsets (Pham et al., 2012). Several approaches try to address this issue by connecting the research components. One approach is called "literate programming", a practice to mix text and code in one dynamic document (Knuth, 1984). Several systems build on this concept: Jupyter notebook combines code and text in one interactive document (Kluyver et al., 2016); RMarkdown combines markdown, i.e. plain text, and $R$ code which can be rendered to PDF files using journal templates (Allaire et al., 2018); and Sweave integrates text written in LaTeX and $R$ code (Leisch, 2002). These documents can be easily shared between collaborators or attached to the paper. A similar approach is StatTag (Welty et al., 2016). Authors can mark code

\footnotetext{
${ }^{1}$ Open Science Framework: https://osf .io/, last access: 01.04.2019.
} 
subsets underlying a specific result in the text, e.g. a number, table, or figure. The resulting linkage enables updates of the result in the text upon changes to the code. StatTag rather addresses authors who would like to work with a dynamic document, but to a lesser extent readers who might want to interact with the results while studying the paper.

In contrast to these solutions, other approaches treat text, data, and code as separate but linkable components. Creating such links can support the understanding of, for example, reviewers who verify the reported results and readers who reuse existing materials (Antoniol et al., 2002). Kauppinen and Espindola (2011) suggest "Linked open data" to connect data, code, and results. Drees et al. (2018) propose using permanent identifiers to connect the paper, data, software, and videos, thereby meeting the "FAIR" ${ }^{2}$ principles (Wilkinson et al., 2016). Still, an extensive workload is needed to understand the source code, which is why Bacchelli et al. (2010) looked at email correspondences among software developers and users. Instead of linking the text to the entire code, they connect code-specific expressions in emails (e.g. classes, functionalities) to the particular section in the source code file. A similar approach was presented by Antoniol et al. (2002) who created fine-grained links between software documentation and the corresponding function and variables. However, scientific articles are fundamentally different from emails or software documentation. Functions in the code are usually not explicitly mentioned in the paper, except they are the object of research.

To address this issue, Pham et al. (2012) developed Sole. Authors can connect a result in the article produced by code to the corresponding code snippet by wrapping the relevant functions into tags. The system SciSoftX offers similar functionality but also provides a UI for readers to examine the selected code and text in parallel (Hoppe et al., 2018). To achieve that, authors create links between code snippets and the paragraph, which are then highlighted in the manuscript. Nevertheless, while Sole and SciSoftX support exploration and understanding of how results were achieved, they lack features which enable manipulation of parameters and reuse of executable code subsets. The approaches presented so far, i.e. research compendia, dynamic documents, and linked materials, pave the way for authors to go beyond the traditional way of publishing research as static PDF files. Interactive and executable papers promise to make research results more easily accessible, to help with understanding them (e.g. using interactive figures), and to facilitate reproduction of computational results.

\footnotetext{
${ }^{2}$ Research data should be findable, accessible, interoperable, and reusable.
} 


\subsubsection{Creating interactive papers}

Increasingly, papers are being published as HTML renderings instead of traditional PDF files. Readers can study the paper as usual but also interact with it in a limited way, e.g. jump to references or download figures. Still, the scientific figures in these papers are usually static, although promising interaction possibilities exist, for example, to enable data exploration (Perkel, 2018). This is a meaningful approach because readers can achieve a better understanding of computational results if they are given some control over what is shown in the figure (Wacharamanotham et al., 2015). In addition, interactive figures are also desirable for authors because, according to Greis et al. (2017), authors' analyses of their data might be based on an inaccurate implementation of the model or uncertain data due to, for example, imprecise measurements. For this reason, they suggest attaching sliders to figures which allow users to define a range as input.

Victor designed the Website worrydream, which allows readers to change the assumptions, i.e. the initial configuration of the parameters, underlying the results in the text (Victor, 2018). Based on those changes, texts and figures adapt accordingly. Using interactive figures to convey results is not new but is rarely done in scientific articles (Perkel, 2018). To try to address this, the publisher Faculty of 1000 introduced the term "Living Figures" (Singh Chawla, 2015); they published scientific articles including interactive figures using Plotly (Sievert et al., 2017), a tool allowing readers to pan and zoom into diagrams to see exact values, and it incorporates UI widgets, such as a slider (for an example, see Colomb and Brembs (2014), and Delory et al. (2018)). However, creating these interactive elements is still a time-consuming task and done by journal staff or in the context of experiments. Creating interactive figures is also possible with "literate programming" approaches. With few lines of code, users can attach UI widgets to figures embedded in Jupyter notebooks using ipywidgets (Mease, 2018), or in RMarkdown documents using Shiny (Chang et al., 2018) and htmlwidgets (Vaidyanathan et al., 2016). Tools such as Vega lite (Wongsuphasawat et al., 2018) allow authors to provide links to external interactive figures.

On a rather programmatic level, tools such as Plumber (Trestle Technology, 2018) and OpenCPU (Ooms, 2018) allow users to create APIs out of source code. Thus, common API requests (GET/POST) can be created to re-run the code with a different parameter value. Software engineers gain full control of how to enable users to manipulate a parameter and how to visualize the returned outcome (e.g. a figure).

In summary, several conceptual and technical solutions support authors in publishing open research. However, even if the computational results are accessible and reproducible, the analysis might not be transparent (Goecks et al., 2010). The code 
and data can still be complex, making it difficult to understand how an individual result, e.g. a specific figure, was computed, which parameters were used, and how changes to these affect the outcome. Bindings address this issue by creating fine-grained linkages between an individual result, the data subset, and code lines. We can use this information to create interactive papers which allow readers to manipulate the initial parameter values used to produce a figure, for example, by using a slider or radio buttons.

\subsection{Binding}

In order to facilitate access to the research components underlying the computational results in a scientific article, we propose the bindings concept. In the following subsections, we first provide a rationale for the design and then describe the key components of bindings.

\subsubsection{Design rationale}

The development process was informed by the needs of several stakeholders who were mentioned in chapter 4: authors and readers; publishers and libraries; and software developers. Authors and readers want to describe and inspect the connections between results, data, and analysis. In order to enable this, a binding has to contain information about which individual computational result is addressed by it. Additionally, a binding needs to include references to the source code lines and data subsets used to produce that result. A further desirable feature for authors and readers is to be able to manipulate the parameters underpinning a result and then to re-compute them using the changed values. To facilitate this, a binding needs to include information about what parameters can be manipulated within what range and a reference to a UI widget that enables users to change the parameter. The description of the dataset finally enables readers to substitute the underlying dataset by their own compatible one.

Publishers and libraries are the second set of stakeholders with needs that impact the design of the bindings. Both maintain infrastructures to host journals or to archive scientific articles and the attached research materials. In order to facilitate the integration of a binding into these infrastructures, it needs to be encoded in a format that is standardized and easily manageable. Encoding bindings as a JSON object which can be part of a paper's metadata or transformed into other formats (e.g. XML) helps to meet these requirements. 
Finally, we also considered software developers who engineer and operate web applications for publishers and libraries. Software developers need to design and implement the UI for authors to create a binding and for readers to use the resulting interactive figure. The implementation of a binding does not require a specific technology (e.g. Plumber) and thus could be different from what we suggest in this work. However, the results should be based on open data formats (e.g. GeoJSON, .csv) to avoid the need for licensed software to display and reuse closed data formats.

In the following subsections, we elaborate on the individual components of a binding in detail. Figure 6.1 shows an example binding containing the elements mentioned above (computational results, code links, UI widgets, and references to data).

\subsubsection{Computational result}

A binding contains a unique reference to the corresponding computational result. This result can be a figure, table, or number in the manuscript, i.e. the source file (e.g. RMarkdown) used to render the PDF or HTML file. In the case of a figure or a table, it is sufficient to include the number (e.g. "Figure 1") which is unique throughout the document and always refers to the same result. Referring to a numeric result in the manuscript is more complex and prone to being ambiguous. For example, the number " 5 " in a text might be a numeric result or a chapter number. For a distinct identification of this result, the binding specifies its location in the article. This requires the file name (e.g. "analysis.Rmd"), the line number in that file, and a short textual paragraph, in which the numeric result occurs only once, as the same number might appear multiple times in the same line. The most fine-grained binding refers to an individual computational result. However, the same code and data described in a binding might produce several numbers, tables, and figures. In this case, a binding might refer to multiple results. Figure 6.1 shows an example binding for a computational result of the type "figure" and the actual result "Figure 3". The remaining components are described in the following subsections.

\subsubsection{Source code}

In order to enable the inspection of source code, a binding specifies which source code lines from which script files are required to re-compute the computational result. To enable one to manipulate the analysis underpinning the result, a binding describes the parameter that should be manipulated, i.e. the name of the parameter, its initial value, and the line number in the corresponding code file. A single binding can contain a set of parameters. The source code lines provided in a binding need to 


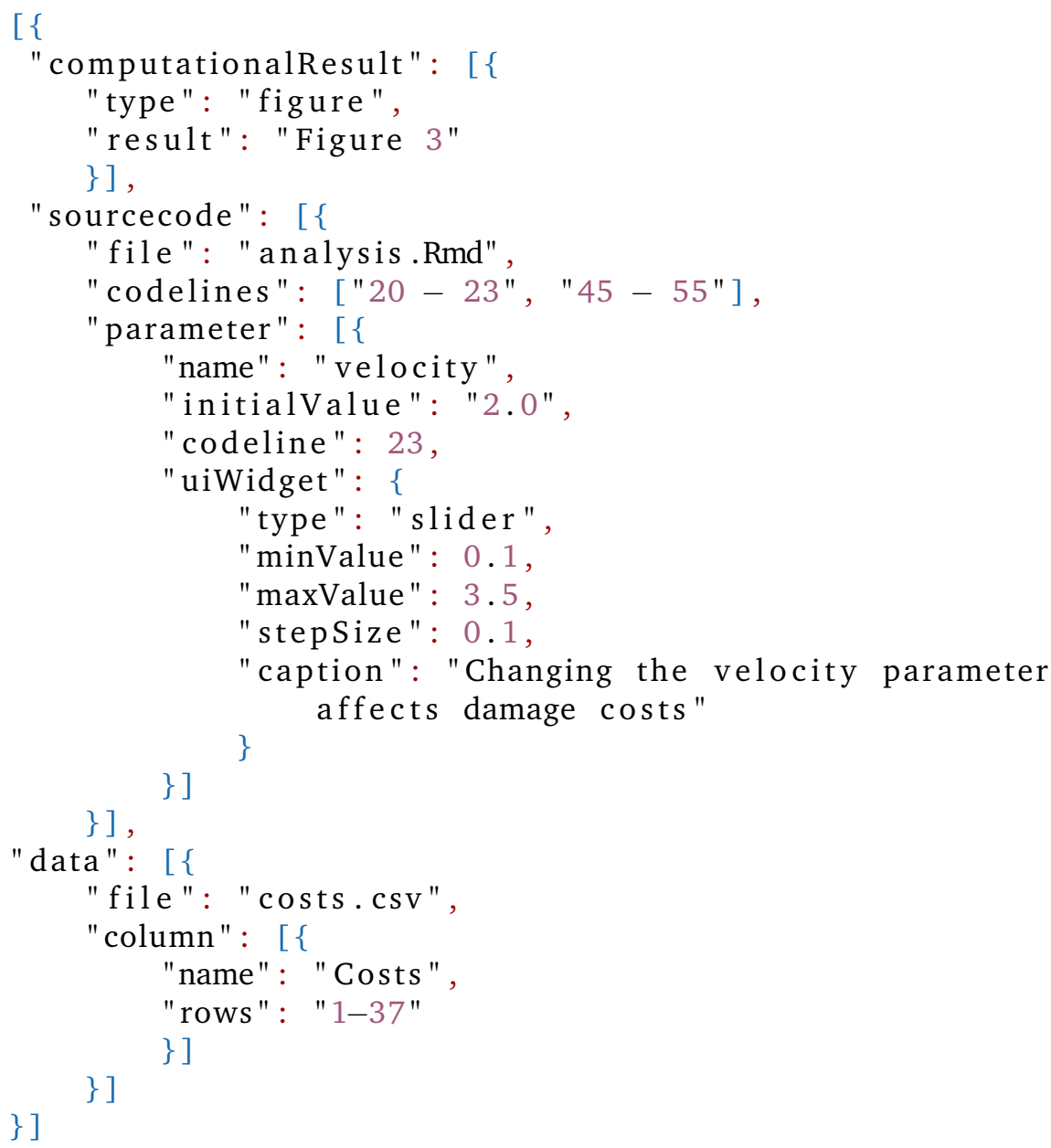

Fig. 6.1: Example binding stored as a JSON object containing the five components computational result, source code, UI widget, and data. The interactive figure that is generated from this binding is shown in Figure 6.3. 
be executable. Otherwise, it is not possible to create interactive figures or to easily reuse the code for one's own work. For this reason, the code lines contain the entire analysis pipeline to re-compute the result. This includes the importing of libraries, loading and processing of the data, analysis, and the final output function. The example binding in Figure 6.1 shows how the description of the code might look like: It specifies the file "analysis.Rmd" that contains the analysis pipeline for "Figure 3 ", which source code lines are needed from that file ("20-23", "45-55"), and the parameter that should be manipulated, i.e. the parameter "velocity" with the initial value "2.0" in line " 23 " of the file "analysis.Rmd". This set of information allows for extracting the required code lines into an executable file which only produces the specified result. This file can then be executed with the new parameter value or reused by readers for their own work.

\subsubsection{Ul widget}

UI widgets can be used to manipulate the parameter values specified in the source code component. The choice of the UI widget depends on the data type of the parameter, e.g. numeric, text, boolean, and the number of potential alternatives. While sliders are suitable for manipulating numeric parameters in a certain range (e.g. 1-100), radio buttons are more appropriate for changing textual parameters, such as the model used in a functionality (see e.g. the "vgm" functionality in $R$ and the options, e.g. "gaussian", "matern", "exponential"). A binding requires further information about the selected UI widget: For example, a slider needs a minimum/maximum value and a step size, whereas radio buttons require a set of potential alternatives to the initial value. To enable independent manipulation of different parameters in the same binding, one UI widget corresponds to one parameter. UI widgets only manipulate the code underlying a figure and not the dataset, since all computational steps related to data processing (deleting outliers, transformation) should be scripted in the code. Manual data processing should be avoided for the sake of reproducibility (Sandve et al., 2013). The binding instance (Figure 6.1) connects a slider to the parameter "velocity". The range of the slider is described by the minimum value "0.1", maximum value " 3.5 ", and the step size "0.1". Finally, authors can add a caption to explain the purpose of the slider.

\subsubsection{Data}

Usually, the source code runs the analysis on data. A binding specifies which data subsets are needed to re-compute an individual result. To achieve this purpose, a binding contains at least the name of the data file. For papers that are based on results that load data from a library, instead of the file name, the binding can 
then specify which function in the code loads the data. In addition, authors can specify single table columns and rows. This fine-grained information is not required for the execution of the source code. However, a key benefit of a binding is the explicit description of how a certain result was achieved. A further benefit relates to being able to substitute the data underlying the result. In creating their own data to substitute for the original one, readers can avoid common interoperability issues, e.g. with respect to data formats, column names, and data types. The data component is also included in the example binding (Figure 6.1). The computational result is based on the rows "1-37" in the column "Costs" of the data file "costs.csv". The readers consequently know which data format to use, how to name the columns, and how many rows the table should have.

\subsection{Realization}

In order to demonstrate the technical realizability of a binding, we implemented a prototypical tool for authors to create a binding, and for readers to use the resulting interactive figure while studying a paper. A video showing all steps is available under https://osf .io/9hb5p/?view_only=b39a75f5c6394c848907f2c49278243c (last access: 01.04.2019).

\subsubsection{Author's perspective: Creating a binding}

Figure 6.2 shows the UI for authors to create a binding. The example illustrates the creation process based on the paper by Dottori et al. (2016). The paper contains a reproducible figure which calculates flood damages based on several parameters, such as flood velocity and duration. According to the authors, changes to the velocity parameter affect the damage calculation. It is thus a suitable candidate for an interactive figure whereby the initial value set by the author can be manipulated. Five steps are required to create the binding as formalized in Figure 6.1.

Step 1: The author specifies the computational result that should be addressed by the binding.

Step 2: Then, the author marks only those source code lines that are required to produce the corresponding result including the import of libraries and data, data processing, analysis, and the output function (see Figure 6.2, top).

Step 3: Next, the author selects the parameter that should be manipulated by marking it in the code subset (Figure 6.2, bottom). The selected parameter and its initial value are shown on the right.

Step 4: Afterward, the author configures the UI widget. Figure 6.2 (bottom) exemplifies the configuration of a slider having a minimum/maximum value and a 

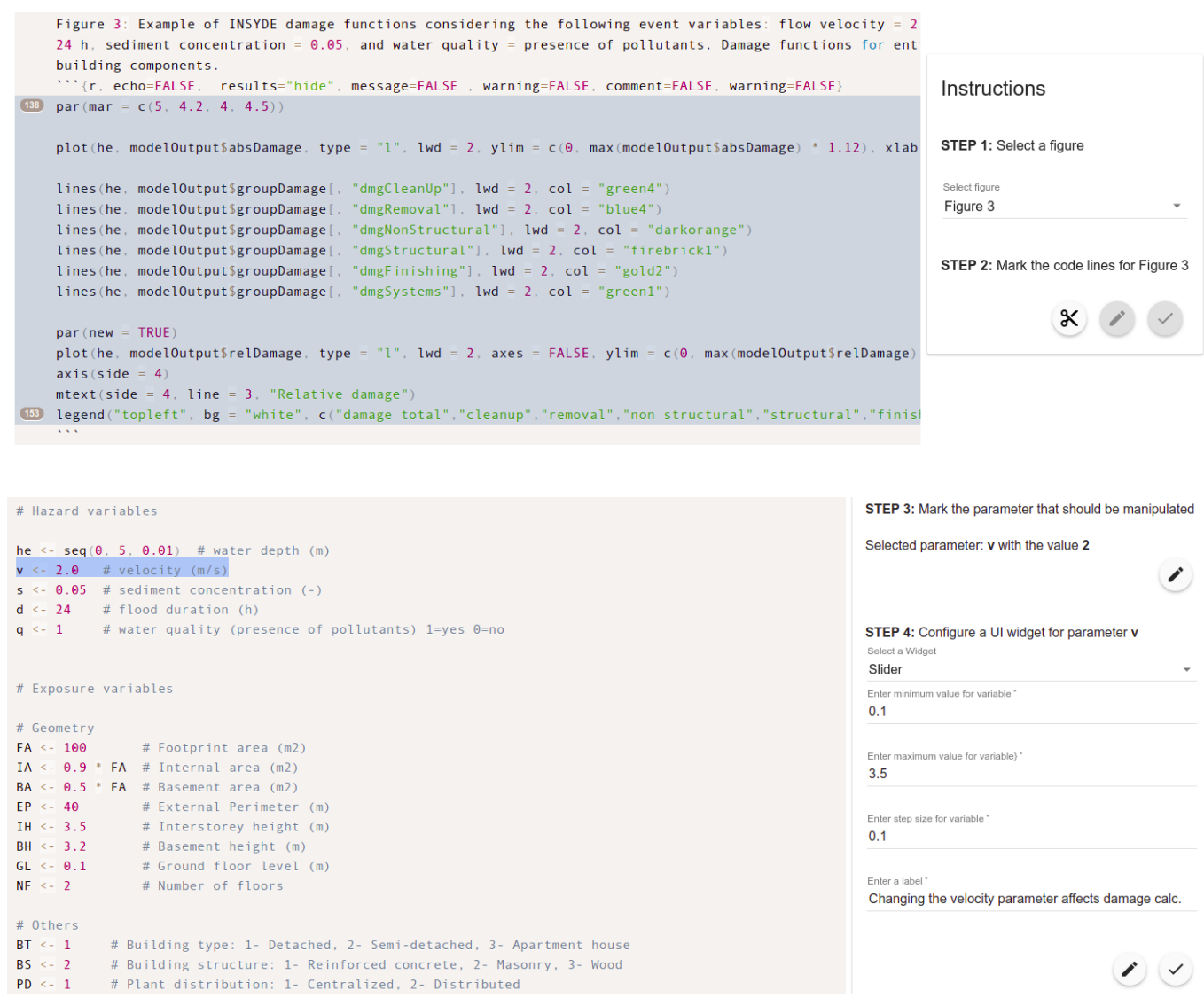

Fig. 6.2: Creating a binding in five steps. Top: Selecting the computational result and the source code lines which produce it (Step 1 and 2). Bottom: Specifying the parameter that should be manipulated and configuring the UI widget (Step 3 and 4). In the fifth step (not shown), authors specify the data subset (see video).

step size. Additionally, the author can add a caption to explain the interactive figure. Step 5: In a final step, the author indicates which data subset is required to produce the specified result, i.e. the file name, columns, and rows.

\subsubsection{Reader's perspective: Using the interactive figure}

Figure 6.3 illustrates the UI for readers, including the interactive figure based on the binding provided by the author. Readers can study the scientific article on the left side of the UI. The computational results for which a binding exists are highlighted in bold. If readers would like to quickly manipulate the original parameter mentioned in the caption of the figure (blue box), they can use the interactive figure on the right side of the UI. Readers can change the parameter "velocity" in order to see how different values affect the damage calculation. A short description at the top of the interactive figure indicates the parameter that can be manipulated, its initial value (green box), and the range of the slider. Below, readers can use the slider. The new parameter value is visible on the right of the slider (black box). The figure adapts each time the reader changes the parameter value. 

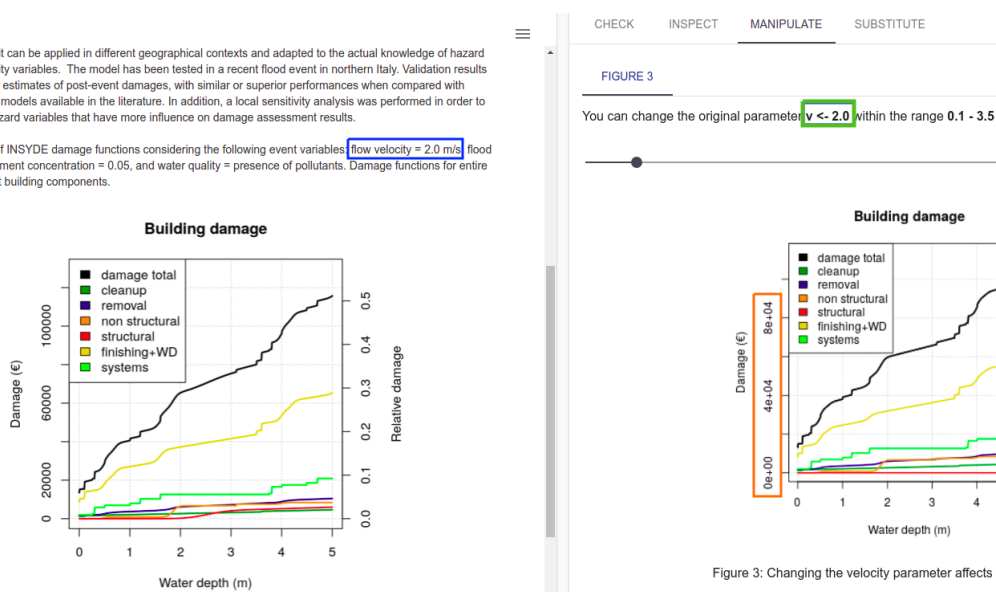

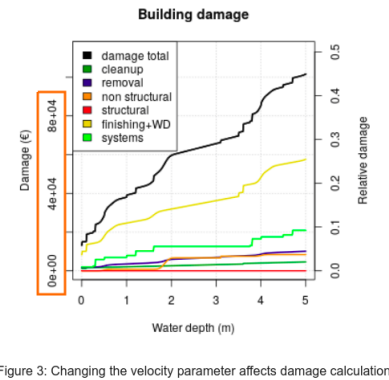

INSPECT CODE AND DATA

Fig. 6.3: In parallel to reading the actual article on the left, readers can also change the original parameter (blue box: "velocity $=2.0$ ") by using the slider on the right and see how the new value (black box: "0.4") affects the output (orange box: "damage costs").

The reader can then compare the original with the manipulated visualization. In addition, readers can inspect the code and the data underlying the particular figure by pressing the button "Inspect code and data" below the figure.

\subsubsection{Technical details}

We built the tools on top of the open source web application presented in chapter 5. The implementation of the frontend tool $^{3}$ is based on AngularJS ${ }^{4}$ (a JavaScript framework) and MaterialUI ${ }^{5}$ (a UI framework). For the backend service ${ }^{6}$ we used node.js ${ }^{7}$ (a server-sided platform for creating web servers), Docker ${ }^{8}$ (a software for encapsulating applications), and Plumber $^{9}$ (an $R$ package for transforming $R$ code into a web api).

\subsection{Evaluation}

In order to gain initial insights into the applicability of bindings and the effort required to create them, we produced interactive figures from existing scientific

\footnotetext{
${ }^{3}$ o2r-platform: https://github.com/o2r-project/o2r-platform, last access for this and the followingURLs: 01.04.2019.

${ }^{4}$ AngularJS: https : //angularjs .org/

${ }^{5}$ MaterialUI: https://material angularjs .org/latest/

${ }^{6}$ bindings service: https://github.com/o2r-project/o2r-bindings

${ }^{7}$ Node: https://nodejs.org/en/

${ }^{8}$ Docker: https://www.docker.com/

${ }^{9}$ Plumber: https://www.rplumber.io/
} 
papers. The materials underlying the results in this chapter are accessible as an Open Science Framework project: https://osf .io/9hb5p/ (last access: 01.04.2019).

\subsubsection{Materials}

We used a subset of the paper corpus described in the reproducibility study mentioned in chapter 3. The 41 papers contained therein are all open access, and they come with code and data attached. From these, we excluded 9 papers since we were not able to execute the source code. Additionally, we excluded 8 papers, because they did not produce figures which were included in the original paper. For practical reasons, we did not consider one paper which had an execution time longer than one hour ${ }^{10}$. Overall, we included 23 papers in our evaluation; 15 of these come from the geosciences and were published by Copernicus Publications ${ }^{11}$ and the remaining 8 describe statistical software for the $R$ programming environment and were published in the Journal of Statistical Software ${ }^{12}$.

\subsubsection{Procedure}

Our first goal was to obtain insights into the applicability of bindings to existing scientific articles. For each paper, we first identified those source code lines that were used to produce an individual figure in the paper. For this purpose, we searched for the plot function which created the figure and then backtracked the source code until we identified only those code lines that are required to produce the particular figure. We thus reduced the entire source code to a smaller but executable code subset, including the import of libraries and data, data processing, analysis, and the visualization of the results. To find out how to display the data employed in the analysis, we also noted the file formats, which indicate the structure of the data. In order to create interactive figures, we then searched for parameters which can be manipulated, for example, using a slider or radio buttons. We focused on parameters which influence the results shown in the figure, e.g. the numbers, curves, or statistics, and not the design, e.g. the color. These parameters and the alternative values should be selected carefully, as changes to the initial configuration might not be meaningful. Consequently, we determined the parameters and the alternative values as follows: First, we scanned the paper and the source code in order to find paragraphs or code comments which discuss parameters used in the code and potential alternatives to their initial configuration. If we were not able to find potential candidates, we examined the functionalities used in the code and consulted the corresponding $R$

\footnotetext{
${ }^{10}$ Laptop: Dell, Ubuntu 16.04 LTS 64 Bit, Intel@ Core $^{\mathrm{TM}}$ i7-6500U CPU @ 2.50GHz x 4, 15,6 GiB RAM.

${ }^{11}$ Copernicus Publications: https://publications.copernicus.org/, last access for this and the following URL: 01.04.2019.

${ }^{12}$ Journal of Statistical Software: https : //www . jstatsoft. org/index
} 
documentation ${ }^{13}$ to find parameters and alternative options for the initial values. If this was again unsuccessful, we tried to find alternative parameter values by looking into the used dataset. If this was also not successful, we considered the figure as not being suitable for manipulations and skipped it. Once we found one suitable parameter and at least one potential alternative, we continued with the next figure in the paper. Afterward, we created Executable Research Compendia (ERCs) (see chapter 4) out of the paper and the materials. First, we encapsulated the materials into a folder and then uploaded it to a web application ${ }^{14}$ that is capable of handling ERCs. Afterward, we used the supportive interface for authors (see above) to create a binding, i.e. we extracted the relevant code lines, selected the parameter that should be manipulated, and configured the UI widget. We skipped the step to specify the dataset, as this information is not needed to re-compute the analysis. Finally, we checked if the creation of the interactive figure based on the binding was successful by using the tool for readers (see above).

Our second goal was to investigate the effort required to create and use bindings. For this purpose, we counted the source code lines (excluding comment and empty lines) and connected code blocks in each binding that we created. A connected code block is a sequence of required source code lines (e.g. a functionality) which can be marked at once until an irrelevant code line emerges. These two measures provide initial insights into how many clicks an author has to perform to create the code subset. In addition, we measured the execution time for each figure to see how long readers have to wait to see the output. A similar performance test was done by Pham et al. (2013) who observed faster execution times if only parts of the analysis were re-executed. In order to measure the execution time, we set a time stamp at the beginning and the end of the analysis. Changes to the parameters might result in very different execution times. As measuring the time for each potential alternative was not realistic, we executed the code with the original parameter value to receive an initial estimate.

\subsubsection{Results}

\section{Parameters}

We excluded 3 papers since they did not contain figures based on parameters. From the remaining 20 articles, we excluded 30 figures which were not based on parameters and 4 figures which required extensive changes to the original source code to access them individually. In total, we created 83 interactive figures. Figures composed of sub-figures (e.g. "Figure a", "Figure b") were counted separately. For

\footnotetext{
${ }^{13}$ R Documentation: https://www.rdocumentation.org/, last access for this and the following URL: 01.04.2019.

${ }^{14}$ o2r-platform: https ://github.com/o2r-project/o2r-platform
} 
these figures, we identified 40 different parameters which can be manipulated. Out of the 40 parameters, we identified 30 by scanning the paper, 5 by reading the corresponding $R$ documentation of the functionality used in the code, 3 by our own examinations, and 2 by comments in the source code made by the original author. For the 40 parameters, we identified alternative values by studying the paper (in 18 cases), by reading the corresponding $R$ documentation (8), by looking into the dataset (8), and from the comments in the code (4). Two parameters were confidence intervals and thus had inherent alternative values, i.e. values between 0 and 1. We distinguish the following three parameter types: Assumption parameters are values that describe the initial configuration of the analysis, e.g. the velocity of the water in a flooding scenario. These parameters are set by the analyst. Out of the 40 parameters, 16 were categorized as assumption parameters. Method parameters specify a method used as part of a functionality, such as the model used to compute a variogram ${ }^{15}$ (e.g. exponential, gaussian). These parameters have predefined values described, for example, in the $R$ documentation of the corresponding function. We identified 15 parameters as method parameters. Three of these changed the visualization of the figure, e.g. from a line to a point diagram. Filter parameters specify the data subset used for the analysis, such as a specific column or values measured within a certain period. Changing these parameters consequently results in running the analysis on a different data subset. We found 9 filter parameters. From the 40 parameters, 21 were textual parameters or, in four cases, only had few numeric alternatives. We thus selected radio buttons to manipulate these parameters (a drop-down list would have been possible, too). The remaining 19 parameters could be changed within a certain range. We thus selected a slider to manipulate these parameters.

\section{Execution times}

On average, the execution time of the entire analysis took 130 seconds for the first run (median $\tilde{x}=25$ secs., standard deviation $\sigma=241$ secs., values ranged from 1-910 secs.). The second run, i.e. with some parts of the code potentially being cached, took 123 secs. on average (median $\tilde{x}=18.50$ secs., standard deviation $\sigma=232$ secs., values ranged from 1-908 secs.). On average, the analysis of a single figure took 35 seconds (median $\tilde{x}=5$ secs., standard deviation $\sigma=117$ secs., values ranged from 1-878 secs.) for the first run, and 33 seconds (median $\tilde{x}=3$ secs., standard deviation $\sigma=115$ secs., values ranged from 1-881 secs.) for the second run.

\footnotetext{
${ }^{15} \mathrm{vgm}$ function in $R$ : https://www.rdocumentation.org/packages/gstat/versions/1.1-6/ topics/vgm, last access: 01.04.2019.
} 


\section{Effort for authors}

In order to create figures that can be manipulated using a slider or radio buttons, we had to make a few changes to the original source code: In the code of 41 out of 83 figures, we had to wrap the output function into a generic plot function (i.e. "plot()" in $R$ ), because our software was not able to handle the diversity of existing plot functions. In the code of 33 figures, we had to put the parameter value into a variable. In the original code, the value was passed directly to the functionality. The step was needed to make the parameter and the value uniquely referable and explicitly available in the binding. Having only the value might lead to ambiguous references, for example, if the same value was used multiple times. In one paper, the code of 9 figures was based on a variable which we had to make globally accessible. Finally, in the code of 2 figures, we had to transform the parameter into another data type. In the code of 35 figures, no changes were required. On average, the entire code underlying a paper had 336 lines of code (median $\tilde{x}=135$, standard deviation $\sigma=529$, values ranged from 36-2199). The code of a single figure was composed of 245 lines of code on average (median $\tilde{x}=28$, standard deviation $\sigma=545$, values ranged from 4-1938). This means that, in the worst case, authors need to mark 245 single source code lines. However, usually several lines of code can be summarized to a connected code block, i.e. continuously connected lines of code, such as a function, resulting in a lower number of clicks. On average, a binding had 9 code blocks (median $\tilde{x}=7$, standard deviation $\sigma=8$, values ranged from 1-35). This means that authors have to mark 9 code blocks on average to create a binding. However, the high standard deviations, particularly regarding the number of code lines, show that the numbers are only rough estimates.

\section{Data}

The data formats used for the analyses underlying the results are diverse. From the 23 articles, 15 included datasets that were stored in open data formats, i.e. csv (9 papers), txt (8), 14c (1), asc (1), and dat (1). Users can inspect these files without additional software. In 9 articles, only open formats were used for the analysis. Out of the 23 articles, 14 contained binary files, i.e. Rdata (4), rds (2), shapefile (2), and sav (1). In addition, 7 datasets were loaded from an $R$ package by calling the name of the dataset in the code. It is not possible to indicate datasets coming from a package the way we did in the example binding above. Instead of the data file, the data component in a binding specifies the name of the dataset and, if applicable, the columns and rows. In the case of binary files, readers need additional software to see the content. In 8 papers, only binary files were used for the analysis underlying the results. 


\subsection{Discussion}

The results of the evaluation provide initial evidence that bindings are technically feasible and can be applied to a range of scientific articles. From our sample composed of 23 papers, 20 included figures based on parameters that can be manipulated with the help of UI widgets. The 20 articles included 30 figures which did not have parameters suitable for manipulations. However, in total, we found and successfully implemented 83 interactive figures which can be manipulated using a slider or radio buttons.

\subsubsection{Parameters}

It is crucial to select the parameters carefully. We tried to avoid meaningless manipulations by studying the paper, comments in the code, and the $R$ documentation. From the 40 parameters, we selected 37 based on these three sources. From these 37 cases, the original author discussed the parameter in the paper or the code in 32 cases and suggested alternative values in 22 cases. These parameters are probably the most suitable candidates for manipulations and show that there might be some need for interactive figures. Nevertheless, consulting the documentation of a specific functionality might result in parameters that support the understanding of how a function works, for example, as part of a teaching class. Since we were not the authors of the selected papers, identifying parameters and value ranges required some effort on our part, which would not be the case if authors (as domain experts) picked the parameters themselves.

We subdivided the parameters into three categories: Assumption parameters were "invented" by the original author, for example, as part of a model. Reviewers and readers might ask about the role of these parameters in the model and how it affects the final result. In contrast, method parameters are predefined, for example, in the corresponding software documentation. The question arises why a certain initialization was selected and how alternative values change the output. Finally, filter parameters specify the data subset used for the analysis and were also defined by the author. Readers and reviewers should know about these parameters to assess whether the overall model is valid. Bindings can help to answer these questions about parameters, for example, by providing means to manipulate them. The 40 parameters can be manipulated using a slider or radio buttons. In practice, the choice for a suitable UI widget would be made by the original author which might be different from what we have selected. 


\subsubsection{Effort and user interface}

The UI showed the process of creating and using a binding, and also delivered initial insights into the required effort. Fortunately, only a few changes to the original source code were needed, such as wrapping a specific output functionality into a more generic one. This might be a reasonable amount of work for authors to make their source code ready for creating interactive figures based on bindings. On average, we had to extract 245 lines of code in 9 code blocks. This number is not high but might constitute a burden for authors to invest time. A (semi-)automatic code extraction might be one way to overcome this barrier. In addition, if authors were aware of the requirements for creating bindings, they could incorporate these into their code from the beginning rather than retrospectively modifying the finished code. Beyond the effort to select code lines, only a few clicks are required to specify a UI widget.

The execution times of the interactive figures provide initial insights into how long readers have to wait until they see the output. This is important because each manipulation requires executing the entire code with the new parameter. Half of the 83 figures took not more than 5 seconds, whereas nine took longer than 60 seconds. Manipulations to the parameters thus rarely led to immediate changes in the figure, which might go against users' expectations. Hence, showing a progress bar, pre-rendering outputs, or catching intermediate results might be needed to avoid long waiting times for readers. While checking the interactive figures using the UI for readers, we noticed that changes in the figure after manipulating the parameter might be difficult to spot. For example, curves in a line diagram only varied moderately. For this reason, readers would benefit from a comparison tool which highlights the differences between the original and the manipulated figure, e.g. with the help of change detection algorithms (Radke et al., 2005).

\subsubsection{Data}

The data used for the analysis might pose a problem. Open data formats, e.g. csv and geoJSON, can be easily displayed in a web application and are readable by humans and machines. In contrast, binary data formats, such as RData or shapefiles, require additional software to make them accessible for users. So far, the implementation does not work with binary data formats and thus might incentivize authors to make use of open formats. In addition, this issue affects the substitution of the dataset underlying an individual computational result. It becomes more challenging for readers to create their own interoperable dataset if the original dataset was stored in a binary file format. However, even in open data formats, the structure of tables (e.g. column names, data types) varies considerably, making it difficult to achieve 
interoperability between datasets (see chapter 5). By specifying the data subset, a binding might support readers in completing this task.

\subsubsection{Benefits}

Bindings are not a necessity for manipulating a parameter in the source code. In theory, an interested reader could take the code, run it locally, and adjust the parameters. However, UI widgets make it much easier to successfully complete this task and allow one to quickly check, for example, how the results change if the underlying assumptions change. This could be the task of reviewers who would like to verify the reported results and to challenge the assumptions made by the author prior to suggesting publication of the paper (Pham et al., 2013). In addition, readers do not have to search for the parameter and to set up the analysis in their local environment. Instead, they can use the interactive figure in parallel to studying the paper. A further benefit of a binding is that it results in executable source code. Instead of writing source code from scratch, readers can reuse the analysis underlying a specific result and build their research on top of it. While this approach is thus beneficial for readers, it requires a way for others to cite the reused code and data (Stodden et al., 2016). Bindings can also contribute to searching for papers in a more fine-grained way. For example, bindings might facilitate finding papers that include results based on code using a certain library.

\subsubsection{Limitations}

The work presented in this paper is subject to a number of limitations. The sample used for the evaluation is small and thus not representative. In addition, we only examined papers with code written in $R$. Further articles from other disciplines based on other programming languages are required to draw more informed conclusions. Nevertheless, the dataset included a variety of figures, which provides an initial evaluation of our approach.

It remains unclear if all parameter manipulations that we applied to the code were meaningful. In practice, parameter and value selection should be made by the original author who knows best which parameters can be manipulated in which way. Nevertheless, most of the parameters and alternative values were chosen because they were mentioned in the paper, in the code, or in the $R$ documentation of the corresponding function. Manipulating parameters is not relevant for all figures based on computational analyses. In our sample, a couple of figures did not have suitable parameters. However, inspecting the underlying code and data, or substituting the data might still be interesting for readers. 
The results regarding the effort to create bindings are only rough measures at this point. The creation of bindings was done by the same person who implemented the prototype. A user study with actual authors might reveal further issues, such as the need for more or less time for creating interactive figures. In our evaluation, we did not consider the time required to spot the source code lines while creating a binding. This was less of an issue for us because we knew the code in detail, and this might also be true for authors who recently finished the analysis. However, the more time that has passed between finishing the analysis and creating a binding, the more time authors might need to become again familiar with their source code. Ideally, both bindings and the code would be created in tandem while authoring a paper. In addition, we did not discuss the fifth step to create a binding in depth, i.e. specifying the dataset. We skipped this step because it is not needed to create an interactive figure which allows for manipulating a parameter. The measures indicating the effort for authors are thus not complete. Hence, further research is needed regarding how much time authors need and if they are willing to invest it for creating an interactive figure. In order to realize bindings, the computational results reported in an article need to be reproducible, which is not yet common practice (Ostermann and Granell, 2017). However, the opportunity to create interactive figures easily based on bindings might incentivize authors to publish open reproducible research.

\subsection{Conclusion}

In this work, we introduced an approach to creating interactive papers which support readers and reviewers in inspecting code and data, manipulating parameters, and substituting the dataset underlying the results in a paper. We proposed the concept of a binding based on the needs of stakeholders, such as scientists and publishers. A key benefit of a binding is that it connects the text with the code and the data at a fine-grained level, which in turn not only simplifies the (manual) inspection of all components but also enables interactive figures. While studying an article, readers and reviewers can use these interactive figures to understand how a specific result was computed and quickly check, how changes to the parameters affect the result. In addition, a binding might foster the reuse of existing code, resulting in more citations for the original author and less work for the reader. This paper thus makes three key contributions: (1) We first presented the concept of a binding. A binding breaks the coarse-grained linkage between the paper, data, and code in order to create fine-grained links between those source code lines and data subsets that are needed to produce an individual computational result (e.g. a figure). On top of that, a binding explicitly specifies the parameters underlying the results and the UI widgets that can be used to manipulate the parameter values defined by the author interactively. (2) Next, we demonstrated the technical feasibility of the concept by implementing a prototypical toolkit which supports the creation and 
usage of bindings. While the implementation showed that only a few steps are needed to create an interactive figure, authors still need detailed knowledge of their code. (3) Finally, we evaluated the approach by applying the binding concept and its implementation to existing scientific papers. We successfully created 83 interactive figures from 20 scientific articles which allow readers and reviewers to manipulate a parameter underpinning the results shown in a figure. In addition, we also identified a number of potential issues, such as long computation times for some figures, and the effort needed to select the relevant code lines. Despite these issues, a key benefit of bindings is that they make it easier for readers and reviewers to explore how an individual result, e.g. a specific figure, was computed, which parameters were used, and how changes to these affect the outcome.

\subsection{Future Work}

The next step is to conduct user studies with authors and readers of scientific articles to collect insights regarding usability and user experience. For this reason, we will improve the current implementation to enable the manipulation of more than one parameter per figure by using different UI widgets. To support the selection of relevant code lines, we will implement a backtrack feature which only requires selecting the functionality that creates the output and not the entire analysis pipeline. We will also implement tools for readers for identifying differences between the original and the manipulated figure, e.g. by using an overlay of both figures or a side-by-side comparison. Based on these advancements, we are also planning to run a study to assess the detailed impact of using interactive papers on readers' understanding compared to traditional publications. 


\section{Discussion}

The main goal of this dissertation was to assist researchers in publishing and using open reproducible research (ORR) in the computational geosciences. To achieve that goal, this thesis investigated the status quo of ORR in the computational geosciences, proposed strategies to increase the number of open and reproducible articles, identified incentives for authors to publish ORR and readers to use it, and finally, presented the bindings concept to create interactive geoscientific papers. The following sections discuss each of these contributions, their implications, and limitations.

\subsection{The status quo of ORR in the computational geosciences}

According to the study results, ORR is rarely practiced in the geoscientific domain. One reason is that many geoscientists are not aware of the principles needed to fulfill ORR (i.e. attaching executable code and data which produce the results from the paper) and instead believe that giving details about code and data is enough. Also, the authors do not see the incentives which warrant the extra effort for preparing the materials. Consequently, the papers frequently do not contain links to the source code and data files underlying the analysis in the paper (see chapter 2). Even when these materials are available, they are rarely executable and often do not produce the results from the paper (see chapter 3). Hence, the "minimum standard for judging scientific claims" (Peng, 2011) is not fulfilled having several implications.

First, reviewers cannot reproduce the figures, tables, and numbers in the article as part of the review process. Instead of being able to verify how the results in the paper were achieved, reviewers have to trust the analysis employed (Bailey et al., 2016). This trust is based upon the documentation in the paper which usually cannot adequately compensate missing source code and data (Gentleman and Lang, 2007). If the analysis is not accessible or executable, the only possibility for reviewers who want to delve into the analysis is to ask the authors for the materials. Hence, missing materials delay the review process since reviewers need to make an inquiry, 
then authors have to respond to it, and finally, reviewers have to investigate the paper again based on the materials. This "detour" causes extra effort which is what scientists usually try to avoid. It remains open how often reviewers, in fact, attempt to reproduce an analysis during the review process. This aspect was not investigated in one of the studies but could reveal reasons for (not) trying to reproduce results and insights regarding how (un)successful reproduction affected their decision to accept or reject a paper.

A further implication is that research results are not sustainable. Other researchers cannot reuse and build upon existing datasets and analyses. Instead, they have to gather data and write source code from scratch which again causes extra work that could be avoided if research results are published openly and reproducibly. In addition to these drawbacks, the resulting extra effort has an impact on the society that collectively provides the funding. Due to the extra effort for scientists, society cannot entirely benefit from the full potential of scientific advancement which would be faster if researchers could reuse existing materials.

Finally, unreproducible papers can hide errors in the analysis since it is not possible to see the detailed computational steps. The past has shown that publishing an erroneous paper can damage the reputation of authors and the credibility of the entire research landscape (Herndon et al., 2014) resulting in the so-called "reproducibility crisis" (Baker, 2016a). A lack of credibility on the part of the public makes it easy, for example, for climate change deniers to doubt research results that prove an increase in global warming. They can simply put the whole paper into question if no materials are accessible that might support the argument in the article. Open reproducible research at least partially tackles these doubts since other researchers and journalists can examine the analysis in detail. They can reproduce the results and verify the outcomes by themselves. Afterward, they can validate the interpretations on the same basis that was used by the authors. However, reproducible research alone cannot ensure that the authors collected the data properly and drew the conclusions correctly. These aspects are part of the related concept "replicability" meaning that others can achieve similar conclusions based on an independent experiment.

Despite the importance of open reproducible research in general, there are some convincing arguments for not publishing materials. For example, fully reproducible research might disclose the geographic location of rare animals and plants from the attached dataset (Powers and Hampton, 2019). Based on this information, others could visit these places and endanger flora and fauna. Another concern is related to ethics (Darch and Knox, 2017), for example, if datasets cannot be anonymized sufficiently thus allowing others to find out the identity of study participants (Narayanan et al., 2016). A similar issue particularly relevant in geographic information science (GIScience) is location privacy. For example, mobile apps might collect location data 
during a long term study (Ataei et al., 2018). Again, publishing this data could reveal the identity of a participant. The solutions presented in this work require all code and data to be visible for inspection by readers and thus do not yet address such issues. One possibility might be to provide tools for authors to hide specific information in the data, e.g. the GPS ${ }^{1}$ tracks. To avoid that authors also hide the unproblematic parts in the data, reviewers can check that during the ERC-based publication process (see chapter 4).

In summary, several reasons (e.g. extra effort, lack of knowledge) prevent geoscientists from publishing open and reproducible research. As a consequence, the lack of the used materials delays the review process and slows down scientific advancement. These shortcomings can be tackled with concrete incentives for authors and readers. Before authors, reviewers, and readers can reap the benefits of ORR, we need to make sure that authors can realize this form of publishing their research results. The next section discusses strategies for assisting geoscientists in adhering to ORR principles.

\subsection{Assisting geoscientists in publishing ORR}

To address the confusion regarding the term "open reproducible research" and what is needed to achieve it, one strategy could be to publish a paper including a definition and concrete steps to achieve it. Such articles exist (cf. Stodden, 2010; Gentleman and Lang, 2007) but the confusion remains, and the papers are still rarely reproducible (see chapter 3 ). Thus, it is questionable if publishing further articles solves this issue. It seems that the suggestions and tools for more "open science" do not reach all (geo)scientists. This issue is not surprising since researchers who are not aware of the topic might not explicitly search for these articles.

Rather than expecting scientists to adhere to a set of reproducibility guidelines, it might be more promising to provide tools which assist authors in publishing ORR. Consequently, this thesis suggested the executable research compendium (ERC) and described its integration into the publication process considering different stakeholders, such as authors, readers, reviewers, and publishers (see chapter 4). The ERC encapsulates data, code, and the entire software environment in a Docker container. The concept of an ERC and its integration into the submission workflow address the drawbacks of "classic" papers mentioned in the previous section. Reviewers can easily reproduce the results ("one-click-reproduce" (Pebesma, 2013)) and are not restricted to only reading the paper. Instead, they can gain indepth knowledge about how the authors achieved the computational results without

\footnotetext{
${ }^{1}$ GPS: Global Positioning System
} 
further delay in the review process. Hence, the review of scientific articles shifts from trusting how the analysis was conducted to understanding it. This understanding can help reviewers to validate better how the authors came to the conclusions in the paper.

Furthermore, other researchers can directly reuse and build upon the results in the paper. They can either reuse data or collect additional data records without thinking about how to structure the data. Instead, they can continue using the data scheme which has already proven to work as part of the analysis. The same applies to the executable analysis which can be either reused directly or modified to fit own requirements. With these advantages, research results become more sustainable. The "recycling" of existing materials is not only beneficial for individual researchers who do not have to start from scratch but also for the entire society who profits by an accelerated scientific advancement.

ERCs can also help to avoid errors in the computational analysis. One error type refers to technical issues as described in chapter 3. In these cases, the papers are not reproducible, and it is difficult for other researchers to reuse materials. One reason is that during the submission process, the authors cannot check whether their scripts are reproducible since all they can do is to attach or link to a folder containing the scripts and data files. Moreover, it is difficult for authors to anticipate future issues, such as deprecated functionalities or a different computational environment of a reader, e.g. concerning the operating system or software version. Despite these issues, the scientific results might still be correct, but it is difficult to assess their validity (Leek and Jager, 2017). The second error type refers to fundamental issues in the analysis or the data and consequently might result in wrong scientific insights. In a "classic" paper without source code and data, it is almost impossible to find these issues. Although the two error types are usually not the result of malicious practice, they can still damage scientific credibility. To avoid such consequences, the ERC-based publication process allows authors to create an executable paper. The authors thus gain the opportunity to find some of the issues by themselves. In a second iteration, reviewers can inspect the analysis and understand how it works. They might find issues in the analysis that were not identified by the author. However, using ERCs does not guarantee error-free papers, but this way of publishing research is more transparent than a PDF file making it easier to detect such issues. This transparency, in turn, eventually increases trust in science and eliminates one point of attack for deniers of scientific outcomes.

Even with the advantages mentioned above, asking authors to submit ERCs instead of traditional formats might be too abrupt. As a result, journals asking for ERCs might receive fewer submissions since authors are not familiar with such a submission type. To overcome the initial skepticism, journals could create a special issue composed 
of ERCs created from already published scientific articles. Potential authors could look at these examples and then know better what to expect. In addition, authors could be accompanied during the submission process by support staff that helps with questions and technical issues. A second possibility is an "evolutionary" integration of ERCs, i.e. ERCs are interactive attachments to the traditional PDF thus replacing the previous folder that only contained code and data files. The second approach still requires authors to create an ERC but also allows submitting a PDF file as they did before. Once the authors become familiar with creating ERCs, journals might consider replacing PDFs by ERCs entirely.

To conclude, executable research compendia assist authors in publishing open and reproducible research. This form of publishing scientific output tackles the implications of unreproducible research: ERCs allow in-depth examination and a better understanding of the analysis by reviewers and readers; researchers can reuse the materials in an ERC resulting in more scientific output; it is easier to detect issues in the analysis before publication which helps to increase the credibility in scientific insights. The concept of an ERC primarily addresses future papers but could also be used for any already published paper as long as source code and data are openly accessible and executable. Despite these advantages, the actual creation process of an ERC likely requires some additional effort from authors, e.g. for making the code executable. According to the findings in chapter 3 , it is unlikely that authors are willing to invest extra time into the creation of executable papers without clear benefits. Rather than "forcing" scientists to adhere to ORR principles, incentives are required that act as rewards for good scientific practice.

\subsection{Incentives based on open reproducible research}

Compared to a "classic" paper, it seems that authors need to invest extra effort to create ERCs and readers need more time to study the content of an ERC. For this reason, it is important to point out concrete incentives provided by ERCs. Ideally, these incentives not only outweigh the extra effort but make authors' and readers' work more efficient.

Being able to reproduce the results reported in a paper is a clear benefit, but having access to code and data in an executable way involves further opportunities. The additional opportunities identified with the help of the study participants resulted in an extended workflow for readers (see chapter 5). It comprises the steps to discover, inspect, manipulate, and substitute research materials underlying the results in a paper. These steps are not contrary to the traditional way of studying a paper but rather enrich common practices. The features described in the step discover refer to finding relevant papers. Since papers published as ERCs contain additional metadata 
(e.g. spatiotemporal information), they can make an author's work better findable and readers can search for papers more efficiently. For example, readers can query papers that apply a specific library even if this information is not mentioned in the actual text of the paper which is what keyword-based search engines usually scrutinize.

The advanced functionalities described in the step inspect make it easier for authors to convey their insights to reviewers who aim at validating the conclusions or to readers who want to build upon the results. This form of inspection is more complicated with traditional papers since readers have to download the attachments (if available), open the files in the local programming environment, find out how to run the code, and understand potentially large source code and data files. Another limitation of this approach is that the three components paper, source code, and data are largely disconnected. In contrast, when using ERCs, readers can benefit from readily executable papers and study only those code lines and data subsets that were used to produce a specific result. Moreover, the implementation of the workflow showed that reading the article and inspecting the three research components are possible in a combined and not separated activity.

A similar benefit emerges during the workflow step manipulate which allows readers to quickly change the parameters of the analysis and understand how different values affect the output. Again, this task takes more time when using papers published as PDFs. Besides downloading and finding out how to run the attached files locally, readers then need to search for the parameters and change the initial parameter values manually. To facilitate this task, ERCs can include interactive figures which allow changing the parameters using UI widgets without the need to bother about code execution. The manipulation of parameters is not only beneficial for readers but also for authors. They can create interactive figures before submitting an article to try out different settings by themselves. In addition, the authors can create an interactive figure to anticipate further inquiries from reviewers who would like to see if different parameter settings result in deviating results.

Being able to substitute the data or the code increases the sustainability of research results. For example, the authors can reuse their own analysis or data for a follow-up paper. Since other researchers do not need to re-implement the analysis from scratch, they can directly build upon the work. The reuse of materials might pave the way for collaborations which is, in turn, beneficial for the original author.

To sum up, ORR and ERCs come with a number of benefits for authors and readers. Some of these potentially tackle the frequently mentioned "fear of being scooped", i.e. authors lose their competitive advantage since others might "steal" their data or analysis (see chapter 3). The extended workflow demonstrated that authors instead 
receive a competitive advantage than lose it when using ERCs. They can make their research better findable since ERCs contain more metadata, better understandable because all materials used to produce the results are accessible, and better reusable since the overall effort to build upon the data and the analysis is low for readers and the original authors. As a consequence of these incentives, authors can save time (e.g. during the review process or follow-up publications), receive a higher amount of scientific output, and an increased number of citations which is reflected in their research impact. This effect might be multiplied if the members of an entire research group work on similar topics and apply ERCs.

\subsection{Creating interactive geoscientific papers using bindings}

The workflow steps discussed in the previous section require interactive user interfaces which allow readers to discover, inspect, manipulate, substitute, and compare the research results. These interactions are hardly achievable with static PDF files. In order to realize interactive geoscientific papers, connections between the source code, data, and the paper are needed. Chapter 6 presented the concept of a binding which links only those code lines and data subsets that were used to produce a specific computational result. A binding can also contain details on UI widgets to realize interactive figures as discussed in the previous section. Readers can, for example, click on a figure to inspect the code and the data that produced a figure, number, or table. In addition, they can manipulate parameters to see how different values affect the final output.

One might argue that this feature is "nice to have" and of interest only to those who would like "to play around" with the results. However, from the investigated papers, many discussed and suggested alternative options for the initial parameter settings. Thus, parameters seem to play a crucial role and frequently have a substantial influence on the analysis. Moreover, the output has implications on the conclusions which were drawn by the author on top of the analysis. Hence, different outcomes might eventually affect the message of the article in different ways. For example, substantial differences in the results after slight changes in the parameter set could call the whole paper into question. In contrast, moderate effects in the result after manipulating a parameter might indicate that the results are robust to changes in the initial assumptions.

The current way of publishing research, i.e. as static PDF files, does not appropriately convey the effects of alternative parameter values understandably. Authors might need several paragraphs to explain how different parameter settings change the results which might become an issue in outlets with length restrictions. In such cases, attaching the materials is not an ideal solution since readers would need to 
find out how to run the code with the new parameter manually. Although this is in principle possible, chapter 3 described technical obstacles that frequently impede these attempts. The concept of a binding can help to overcome this issue since it contains all information required to re-compute a specific result. Nevertheless, this is only true if bindings are embedded as part of ERCs (see chapter 4) which include a specification of the entire software environment. An implementation outside ERCs is technically possible but might result in issues related to software versions (see chapter 3 and 4).

The presented tools for creating bindings primarily addressed the authors of a paper. Nevertheless, reviewers and readers might be interested in manipulating further parameters or in re-computing the analysis with a value outside of a range pre-defined by the author. Such ideas could emerge during the review process to check the robustness of the analysis, or while reusing the analysis. The concept of a binding supports these use cases. The implementation could provide the same tools for readers who can either modify existing bindings or create them by themselves from scratch. However, such new or modified bindings should be used carefully and not published without explicit confirmation by the original author.

The bindings concept does not take into account semantics, i.e. the meaning of the text in a paper. This means that the new output which resulted from the manipulation of a parameter might become inconsistent with the text. For example, the results section might say that a value $A$ is larger than a value $B$. A manipulation of the analysis might turn this proportion around, but this is not reflected in the text since it does not update accordingly. Readers have to be made aware of this issue while they manipulate a parameter of an analysis. Since bindings also contain a reference to the text, the corresponding text snippet could be highlighted saying that it might have become inconsistent with the new output. In order to avoid confusion, it might make sense to implement a UI which provides a separate view for the actual paper text and the interactive result (see chapter 5). Once the semantics are integrated, it could be possible to let readers interact directly with the text as exemplified by Victor (2018) and Dragicevic et al. (2019).

A similar issue could emerge concerning the conclusions which are based on the analysis. These interpretations at the end of a paper might become inconsistent with the manipulated analysis. Although the concept of a binding does not yet include connections to conclusions, the "results" component in a binding could be extended by a "conclusion" element. Then, authors could not only specify the code lines, data subsets, and UI widgets for a specific result, but also the corresponding conclusion statement that is based on that result. Adapting bindings also to include conclusion statements requires the "conclusion" element as mentioned above and a UI that allows selecting the corresponding statements. A benefit of this approach could be 
that readers not only inspect how a specific figure was produced but also which results led to a specific conclusion. However, conclusions are often interpretations of all results. It remains open whether a single conclusion can be linked to a specific result, but the paper corpus from chapter 3 can help to investigate this question.

All in all, we can note that the bindings concept paves the way for interactive geoscientific papers. Bindings seem to be relevant for papers in the geosciences since many papers report on computational results and discuss alternative parameter values. The fundamental limitations are potential inconsistencies since manipulations are not yet reflected in the context of the text.

\subsection{Generalizability and limitations of the results}

The generalizability of the results in this work is subject to a number of limitations. First, the number of study participants was rather low and thus not representative. Although researchers from different geoscientific disciplines took part, we could not cover all research areas related to the geosciences. The same applies to the two reproducibility corpora, each having rather small sample sizes which likely did not represent the entire spectrum of computational geosciences. The question arises, to which degree we can trust the results. In literature, there is no consensus about how large sample sizes should be (cf. Alroobaea and Mayhew, 2014). Some argue that five participants are enough to find $80 \%$ of all usability issues and that additional subjects rather repeat already identified issues than reveal new ones (cf. Nielsen et al., 2006). Others claim that this number is too low and might conceal issues (cf. Lindgaard and Chattratichart, 2007). It is difficult to tell in advance, how many cultural and technical obstacles exist which prevent geoscientists from publishing and using ORR. With respect to the study results (online survey, interviews, focus group, and reproducibility studies), we can note that several issues occurred multiple times. Hence, we can assume that the studies revealed at least a set of major issues. Nevertheless, additional participants and geoscientific articles might disclose further specific issues that were not discussed in this work.

Besides the generalizability to the geosciences, it remains open to which extent the contributions in this thesis can be helpful for other research fields. Similar to the geosciences, several other disciplines include articles based on computational methods, for example, statistical analyses. Regardless of which scientific domain is taken into account, computational research typically comprises similar tasks: processing data; implementing a model/analysis; visualizing the findings (e.g. in figures or tables). In principle, the strategies, concepts, and tools proposed in this work could be relevant for each of these tasks. The ERC encapsulates the data without explicitly stating what kind of data, but ERCs require the data processing 
to be scripted and not done manually. ERCs are not restricted to datasets with spatiotemporal properties, but might also work with data originating from nongeographic disciplines. However, data from other research areas might also have individual issues, for example, related to ethics. A similar consideration can be made for the source code underlying the implementation of the analysis or model. While the concepts of an ERC and a binding generically refer to code, the implementations have only been applied to papers based on code written in $R$. Nevertheless, this also means that papers from other fields can benefit from this work as long as they use $R$. Tests are needed to prove the technical applicability to other programming languages. Python, for example, is a similarly popular scripting language for statistical analyses but might involve own issues, e.g. related to the installation of libraries. Such issues are related to the software environment specified in an ERC and thus need to be addressed by the containerization technology (e.g. Docker). A further aspect is the applied programming paradigm. The concepts and tools are better suitable for scripted analyses than for complex object-oriented software (e.g. to create geographic information systems) which is usually too complex for bindings. When it comes to visualizing the findings, the applicability of the tools is restricted to two-dimensional figures. In biology, three-dimensional figures which allow to turn and move molecules play an important role (Attwood et al., 2010). The current implementation does not take into account such visualization types since the result of a manipulation is a static picture and not a three-dimensional space. One solution to this limitation is using graphing libraries (e.g. Plotly ${ }^{2}$ ) which allows, for example, panning and zooming within the figure.

Similar to the ERC, the extended workflow and the bindings are not necessarily constrained to geoscientific papers but might also be relevant for other disciplines that use computational methods. The workflow steps to discover, inspect, manipulate, substitute, and compare research materials could be useful for scientists as soon as the analysis in a paper is based on source code, parameters, and data. For example, the search engine $s w M A T H^{3}$ allows discovering papers that mention a certain software in the paper. ERCs can extend such information services since they also contain details contained in the source code files and datasets. After the search for papers that report on specific software, it is not unlikely that readers also want to inspect the materials. The manipulation of parameters might become difficult if the computations take several months because of large datasets or a complex analysis. It is not realistic to pre-render the results based on all potential parameter values since the overall computations would take several years in the worst case. A similar issue might occur during the substitution of the dataset which also requires re-running the analysis. Nevertheless, these cases are beyond the scope of this work as there is still a large number of papers based on rather short computation times (see chapter 6).

\footnotetext{
${ }^{2}$ Plotly: https://plot.1y/, last access for this and following URL: 01.04.2019.

${ }^{3}$ swMATH: https://swmath.org/about_contact
} 
Despite these considerations, a profound statement about the applicability of the workflow to other disciplines requires further research. One possibility is to use the overall approach presented in this work as a blueprint to investigate ORR in other disciplines. The first step would be to gain an initial overview regarding challenges, obstacles, and needs via surveys, interviews, and group discussions. The next steps are creating a corpus of papers that have code and data attached, and trying to reproduce the results from the paper by executing the code. The resulting issues can then be tackled with an approach similar (or equal) to the ERC. Finally, one could study the papers to check if they contain paragraphs about alternative parameter values that can be provided with the help of interactive figures based on bindings. The details about the exact procedures of the different approaches are described in the corresponding chapters and the supplements of this thesis.

The generalizability of the results in this work can also be discussed with respect to author guidelines from funding programs and journals. The research program Horizon 2020, for example, expects applicants to provide a data management ${ }^{4}$ plan to ensure accessibility and reusability. To become published in the journal Ecological Applications ${ }^{5}$, authors have to make their data and source code available in a permanent way. By providing access to executable code and data, ERCs can help authors to fulfill these guidelines easily. Nature ${ }^{6}$ requires authors of papers based on microarray data to make it available on specific data repositories, e.g. ArrayExpress ${ }^{7}$. This requirement speaks against encapsulating the data in an ERC (see chapter 4) since data repositories are usually not able to execute the analysis. One solution is to "open" ERCs by also allowing data storage outside ERCs and to reference the data within the compendium. For the ACM CHI Conference on Human Factors in Computing Systems $^{8}$, the supplemental material is optional and limited to 120 megabytes. A similar constraint is given by AIMS Matehmatics ${ }^{9}$. Since Docker images quickly become large (several gigabytes), storing ERCs is probably not possible within the infrastructure of these outlets. One solution might be to store the ERC on platforms such as Zenodo ${ }^{10}$ which allow larger file sizes (up to 50 gigabytes ${ }^{11}$ ) and to request the content via their $\mathrm{API}^{12}$ on demand.

\footnotetext{
${ }^{4}$ Horizon 2020 data management plan: http://ec.europa.eu/research/participants/ docs/h2020-funding-guide/cross-cutting-issues/open-access-data-management/ data-management_en.htm, last access for this and the following URLs: 01.04.2019.

${ }^{5}$ Ecological Applications: https://esajournals.onlinelibrary.wiley.com/hub/journal/ 19395582/resources/author-guidelines-eap

${ }^{6}$ Nature guidelines: https://www.nature.com/nature/for-authors/supp-info

${ }^{7}$ ArrayExpress: https://www.ebi.ac.uk/arrayexpress/

${ }^{8}$ CHI: http://chi2019.acm.org/authors/papers/

${ }^{9}$ AIMS Mathematics: http://www.aimspress.com/news/204.html\#File

${ }^{10}$ Zenodo: https://zenodo.org/

${ }^{11}$ Zenodo policies: https://help.zenodo.org/

${ }^{12}$ Zenodo API: https : //developers. zenodo.org/
} 
One essential limitation of this work is the lack of an evaluation of the identified incentives. In several sections (cf. chapter 6), this thesis refers to the possibility of gaining an increased understanding if research is open and published as an ERC including bindings. These tools might assist authors in the complex task of conveying research understandably. However, it remains open what exactly is meant by that understanding, to what extent reviewers and readers receive additional knowledge during the workflow steps, and how much time it takes to understand what is conveyed by ERCs and bindings. A user study that investigates these aspects might result in a more informed incentive for authors to publish reproducible research. For this reason, the future work section 9.2 suggests potential approaches to measure the understanding gained while studying interactive geoscientific articles.

Besides the possibility to convey comprehensible research, this work identified further incentives for authors, e.g. an increasing number of articles and citations. It is questionable whether striving for a growing amount of scientific papers is a desired goal. Due to the increasing number of articles that are submitted and published, reviewing these papers and keeping track of them is already a challenging task. This issue is in part caused by the pressure to publish which also gave rise to scientific papers of low quality, for example, because of fraud (cf. Beaufils and Karlsson, 2013). ERCs should not function as a driving force for the "publish-or-perish" mentality (Rawat and Meena, 2014). Instead, since ERCs are transparent regarding analysis and data, they might help reviewers to sort out some of the papers early in the review process. Nevertheless, the evidence that ORR, in fact, results in more research output and impact is still missing. These aspects could be investigated as part of a long-term study where authors submit ERCs to a journal (see section 9.1).

Furthermore, the scope of this work was limited to quantitative data. A large number of research articles report on results based on qualitative data, e.g. interviews and focus groups. Even if these papers do not report on complex geostatistical analyses, it might still be interesting to create ERCs and bindings out of them (Kray et al., 2019). Taking the interviews described in chapter 5 as an example, one might be interested in following the grounded theory approach applied. To facilitate the inspection of the method, a binding could link the quote or a higher-level theme in the data to the author's interpretation in the paper. This approach might increase the transparency of interview results and foster a discussion about reproducibility of qualitative data analysis. Nevertheless, authors usually do not publish interview data in order to ensure that participants are anonymous. Since the number of interviewees is often low, it might be easy to identify individuals based on their statements. Hiding specific information, such as origin, affiliation, age, and gender could in part solve this issue. In addition, the consent form should be adapted accordingly to inform participants about this approach and to ensure ethical compliance (cf. Langheinrich et al., 2013). 


\section{Conclusion}

Open reproducible research (ORR) is not only about being able to re-compute the computational results from the paper using the original source code and data. ORR is a fundamental requirement to maintain high research standards and credibility of scientific insights. However, many scientific publications across research disciplines (including computational geosciences) are not reproducible. This thesis explored different aspects of ORR in the field of computational geosciences, i.e. why geoscientific papers are not reproducible, what is needed to assist authors in adhering to ORR principles, which opportunities result from executable papers that motivate authors to publish ORR, and finally, how these opportunities can be realized. As a result, this dissertation provided the following key contributions:

- First, this thesis examined the current state of open reproducible research (ORR) in the computational geosciences. The results include a set of obstacles which prevented geoscientists from publishing ORR (e.g. lack of incentives, fear of being scooped) as well as an assessment of whether recently published geoscientific articles are reproducible (see chapter 3). Overall, the results show that many geoscientific articles are not reproducible. Key reasons are a lack of materials as well as technical issues while executing the code.

- Second, this work proposed the executable research compendium (ERC) and its integration into the publication process as a strategy to assist authors in adhering to ORR principles. The ERC also considers the needs of different stakeholders such as readers, reviewers, and publishers.

- Third, this thesis identified concrete incentives for authors and readers to publish and use ORR with the help of ERCs: Authors gain a competitive advantage since their work is better findable, understandable, and reusable. In addition, these benefits save time, for example, during the review process or follow-up publications. As a result, authors might work more efficiently and achieve a higher research impact by receiving more citations from readers who can quickly build upon the materials. 
- Finally, this work designed, implemented, and evaluated the bindings concept, i.e. fine-grained links between the code and the data that produced a specific result in the text. It can also include UI widgets, e.g. a slider, to create interactive geoscientific papers. The extended workflow and the bindings concept change the way how research is done in general and presented to readers.

All in all, the four contributions assist geoscientists in adhering to high scientific standards and show how publishing ORR can be used to create interactive geoscientific papers. This form of publishing research results is more transparent, helps to maintain the credibility of scientific outcomes, and is more sustainable than research published in "classic" PDF files. 


\section{Future Work}

The results presented in this thesis pave the way for several promising future work directions.

\subsection{Practical work}

Based on the initial implementations that demonstrated the feasibility of the concepts, more advanced prototypes are needed to assist users in interacting with ERCs. The tools for discovering ERCs could also allow searching for papers based on code using a specific functionality. A more robust user interface for inspecting ERCs should also be able to handle different open data formats. To further facilitate the creation of interactive figures, a semi-automatic approach for creating bindings is required (see chapter 6). In addition, tools for comparing the original output with reproduced or manipulated ones might assist readers in spotting differences. To receive insights into the user experience, usability, and acceptance, these tools could then be evaluated using qualitative methods, e.g. interviews, or quantitative measures, such as (standardized) questionnaires and logging.

Furthermore, it would be interesting to integrate ERCs and bindings into the infrastructure of publishers, for example, as part of a special issue. Such an outlet could be created in two ways: First, authors submit an ERC as an interactive attachment to their actual paper. Alternatively, the ERC replaces the "classic" paper and is the item under review. Regardless of which approach is preferred, the technical integration should be realized as a module resulting in several advantages: First, the tools can be attached to the publisher's infrastructure with minimal changes to the existing system and without affecting running systems. Second, the publishers do not have to allocate many resources for the integration process. As a consequence, the modular approach might increase the acceptance of publishers and uptake of service that assist authors in publishing reproducibly. 


\subsection{Research}

As described in the ERC-based workflow (see chapter 5), each of the steps discovery, inspection, manipulation, substitution, and compare might contribute to reviewers' and readers' understanding. It remains open, to what extent users obtain a better understanding. It might be interesting to measure the knowledge gained after each step. One possible approach is the use of concept maps as suggested by Assaraf and Orion (2005) and Ifenthaler (2008). First, the original author creates a concept map that describes the results reported in the paper. This concept map acts as a reference map. In a second step, a reader creates a concept map to capture the knowledge state before reading the paper. Capturing prior knowledge is needed since it is otherwise not possible to identify the increased understanding. Then, the reader extends this map after each workflow step. Ideally, readers can add additional concepts and relationships after each workflow step thus approaching the reference map. The overlapping and differing concepts and relationships in the graph are then analyzed to infer to which extent the reader understood the paper. In parallel, this study could be used to asses the understanding gained through interactive papers compared to static articles. This comparison could be achieved, for example, by having a second group that only uses the static paper as a basis to create concept maps. However, a key issue in this approach is that reading an entire article might take several hours. To overcome this issue, participants might be confronted only with a small section of the paper.

The second approach is aligned to the procedure applied by Wacharamanotham et al. (2015). Two groups A and B start with a test composed of questions to capture prior knowledge. Then, group A reads the static article while group B interacts with the ERC. Afterward, another test investigates the knowledge gained after the two groups finished working with the paper and the ERC. In a second run, group A interacts with the ERC and group B reads the static paper. This task is followed by a final test. All three tests contain the same questions to allow inferences about the development of participants' understanding. This approach could be extended by intentionally integrating errors in the research that readers should be able to find if they understood the research results. Regardless of the used method, an important aspect is the time needed to interact with ERCs. The understanding obtained while studying ERCs might be significantly higher compared to the investigation of static papers. However, this benefit needs to outweigh the invested time which is not given if readers have to spend several additional hours to obtain a slightly better understanding. Ideally, the additional time required to study ERCs is moderate compared to static papers with a considerable increase in knowledge. 


\subsection{Outlook}

To close this work, it might be interesting to take a brief look at future prospects. Kray et al. (2019) envisioned the Open Research Infrastructure for Geoinformatics (OpenRIG). OpenRIG is basically a hub for ERCs which provides tools for working with multiple compendia. Besides tools for discovering ERCs (see chapter 5), such an infrastructure could provide the computational capabilities to execute ERCs, particularly those including complex analyses which are too demanding for a researcher's computer. A typical task of a researcher is to compare multiple papers which is also relevant in context of ERCs. The infrastructure could facilitate this activity by aligning different visualizations types among several ERCs with the help of bindings. A further possibility is an automatic analysis of ERCs, for example, to check if ERCs reporting on similar topics are consistent, or to combine ERCs which use the same/similar data and analyses. Automatic analyses of ERCs might also be interesting for checking whether updates of libraries used in the code affect the final results. Despite the advantages of this vision, the barriers that prevent geoscientists from publishing open reproducible research persist but might be tackled with the incentives proposed in this dissertation. 



\section{Bibliography}

Adnan, Muhammad, Mike Just, and Lynne Baillie (2016). „Investigating Time Series Visualisations to Improve the User Experience“. In: Proceedings of the $2016 \mathrm{CHI}$ Conference on Human Factors in Computing Systems. CHI '16. ACM, pp. 5444-5455. eprint: http: //doi.acm.org/10.1145/2858036.2858300 (cit. on p. 85).

Allaire, JJ, Yihui Xie, Jonathan McPherson, et al. (2018). rmarkdown: Dynamic Documents for $R$. R package version 1.11. eprint: https://rmarkdown.rstudio.com (cit. on pp. 42, $59,108)$.

Alroobaea, Roobaea and Pam J. Mayhew (2014). „How many participants are really enough for usability studies?"In: 2014 Science and Information Conference. IEEE. eprint: https: //doi.org/10.1109/sai.2014.6918171 (cit. on p. 135).

Antoniol, Giuliano, Gerardo Canfora, Gerardo Casazza, Andrea De Lucia, and Ettore Merlo (2002). „Recovering traceability links between code and documentation“. In: IEEE transactions on software engineering 28.10, pp. 970-983. eprint: https://doi .org/10.1109/ TSE. 2002.1041053 (cit. on p. 109).

Assaraf, Orit Ben-Zvi and Nir Orion (2005). „Development of system thinking skills in the context of earth system education“. In: Journal of Research in Science Teaching 42.5, pp. 518-560. eprint: https://doi.org/10.1002/tea.20061 (cit. on p. 142).

Ataei, Mehrnaz, Auriol Degbelo, and Christian Kray (2018). „Privacy theory in practice: designing a user interface for managing location privacy on mobile devices“. In: Journal of Location Based Services 12.3-4, pp. 141-178. eprint: https : //doi .org/10 .1080/ 17489725.2018.1511839 (cit. on p. 129).

Attwood, T. K., D. B. Kell, P. McDermott, et al. (2010). „Utopia documents: linking scholarly literature with research data“. In: Bioinformatics 26.18, pp. i568-i574. eprint: https: //doi.org/10.1093/bioinformatics/btq383 (cit. on p. 136).

Bacchelli, Alberto, Michele Lanza, and Romain Robbes (2010). „Linking e-mails and source code artifacts". In: Proceedings of the 32nd ACM/IEEE International Conference on Software Engineering-Volume 1. ACM, pp. 375-384. eprint: https://doi.org/10.1145/ 1806799.1806855 (cit. on p. 109).

Bailey, David H., Jonathan M. Borwein, and Victoria Stodden (2016). „Facilitating Reproducibility in Scientific Computing: Principles and Practice“. In: Reproducibility. John Wiley Sons, Ltd. Chap. 9, pp. 205-231. eprint: https : //doi .org/10.1002/9781118865064. ch9 (cit. on pp. 59, 127). 
Baker, Monya (2016a). „1,500 scientists lift the lid on reproducibility“. In: Nature 533.7604, pp. 452-454. eprint: https://doi .org/10.1038/533452a (cit. on pp. 2, 22, 38, 41, 55, 128).

- (2016b). „Why scientists must share their research code“. In: Nature. eprint: https : //doi.org/10.1038/nature.2016.20504 (cit. on p. 31).

- (2017). „Reproducibility: Check your chemistry“. In: Nature 548.7668, pp. 485-488. eprint: https://doi.org/10.1038/548485a (cit. on p. 13).

Barba, Lorena A. (2016). „The hard road to reproducibility“. In: Science 354.6308, pp. 142142. eprint: https://doi.org/10.1126/science.354.6308.142 (cit. on pp. 19, 20, 84).

Barnes, Nick (2010). „Publish your computer code: it is good enough“. In: Nature 467.7317, pp. 753-753. eprint: https://doi.org/10.1038/467753a (cit. on pp. 2, 41, 67, 107).

Baumer, Benjamin and Dana Udwin (2015). „R Markdown“. In: Wiley Interdisciplinary Reviews: Computational Statistics 7.3, pp. 167-177. eprint: https://doi .org/10.1002/ wics.1348 (cit. on p. 85).

Beaufils, P. and J. Karlsson (2013). „Legitimate division of large datasets, salami slicing and dual publication. Where does a fraud begin?" In: Orthopaedics \& Traumatology: Surgery \& Research 99.2, pp. 121-122. eprint: https://doi.org/10.1016/j.otsr.2013.01.001 (cit. on p. 138).

Bechhofer, Sean, Iain Buchan, David De Roure, et al. (2013). „Why linked data is not enough for scientists“. In: Future Generation Computer Systems 29.2. Special section: Recent advances in e-Science, pp. 599-611. eprint: https://doi.org/10.1016/j. future. 2011. 08.004 (cit. on pp. 20, 64).

Belhajjame, Khalid, Jun Zhao, Daniel Garijo, et al. (2015). „Using a suite of ontologies for preserving workflow-centric research objects". In: Journal of Web Semantics 32, pp. 16-42. eprint: https://doi.org/10.1016/j. websem.2015.01.003 (cit. on p. 64).

Benestad, Rasmus E., Dana Nuccitelli, Stephan Lewandowsky, et al. (2016). „Learning from mistakes in climate research". In: Theoretical and Applied Climatology 126.3, pp. 699703. eprint: https://doi.org/10.1007/s00704-015-1597-5 (cit. on p. 41).

Bik, Elisabeth M., Arturo Casadevall, and Ferric C. Fang (2016). „The Prevalence of Inappropriate Image Duplication in Biomedical Research Publications“. In: mBio 7.3. Ed. by L. David Sibley. eprint: https ://doi.org/10.1128/mBio.00809-16 (cit. on p. 84).

Bishop, Ann Peterson (1999). „Document structure and digital libraries: how researchers mobilize information in journal articles". In: Information Processing \& Management 35.3, pp. 255-279. eprint: https : / / doi .org/10 . 1016/S0306-4573 (98)00061-2 (cit. on pp. $83,88,102$ ).

Bizer, Christian, Tom Heath, and Tim Berners-Lee (2009). „Linked Data - The Story So Far“. In: International Journal on Semantic Web and Information Systems 5.3, pp. 1-22. eprint: http://dx.doi.org/10.4018/jswis. 2009081901 (cit. on pp. 74, 101).

Bloom, Theodora, Emma Ganley, and Margaret Winker (2014). „Data Access for the Open Access Literature: PLOS's Data Policy“. In: PLOS Medicine 11.2, pp. 1-3. eprint: https: //doi.org/10.1371/journal.pmed.1001607 (cit. on p. 63). 
Boettiger, Carl (2015). „An Introduction to Docker for Reproducible Research“. In: SIGOPS Operating Systems Review 49.1, pp. 71-79. eprint: http : //doi . acm . org/10 .1145/ 2723872.2723882 (cit. on p. 66).

Boettiger, Carl and Dirk Eddelbuettel (2017). „An Introduction to Rocker: Docker Containers for R“. In: The R Journal 9.2, pp. 527-536. eprint: https://journal.r-project.org/ archive/2017/RJ-2017-065/RJ-2017-065.pdf (cit. on p. 50).

Bollen, Kenneth, John T Cacioppo, Robert M Kaplan, et al. (2015). „Social, behavioral, and economic sciences perspectives on robust and reliable science". In: Report of the Subcommittee on Replicability in Science Advisory Committee to the National Science Foundation Directorate for Social, Behavioral, and Economic Sciences 3. eprint: http: //web. stanford. edu/group/bps/cgi-bin/wordpress/wp-content/uploads/2015/ 09/NSF-Robust-Research-Workshop-Report.pdf (cit. on pp. 4, 38, 39, 41, 47, 55, 63).

Borgman, Christine L. (2007). Scholarship in the Digital Age. The MIT Press. eprint: https: //doi.org/10.7551/mitpress/7434.001.0001 (cit. on p. 67).

Brase, Jan (2009). „DataCite - A Global Registration Agency for Research Data“. In: Cooperation and Promotion of Information Resources in Science and Technology, 2009. COINFO'09. Fourth International Conference on. IEEE, pp. 257-261. eprint: https : //doi.org/10.1109/COINF0.2009.66 (cit. on pp. 68, 74).

Brunsdon, Chris (2016). „Quantitative methods I: Reproducible research and quantitative geography“. In: Progress in Human Geography 40.5, pp. 687-696. eprint: https : //doi . org/10.1177/0309132515599625 (cit. on pp. 15, 40, 42).

Buckheit, Jonathan B and David L Donoho (1995). „Wavelab and reproducible research“. In: Wavelets and statistics. Springer, pp. 55-81. eprint: https://doi .org/10.1007/978-14612-2544-7_5 (cit. on pp. 1, 74).

Button, Katherine S., John P. A. Ioannidis, Claire Mokrysz, et al. (2013). „Power failure: why small sample size undermines the reliability of neuroscience“. In: Nature Reviews Neuroscience 14.5, pp. 365-376. eprint: https://doi.org/10.1038/nrn3475 (cit. on p. 13).

CCSDS (2012). Consultative Committee for Space Data Systems, Reference model for an open archival information system (OAIS). Magenta Book CCSDS 650.0-M-2, Open Archives Initiative. eprint: https://public.ccsds.org/pubs/650x0m2.pdf (cit. on p. 74).

Chang, Winston, Joe Cheng, JJ Allaire, Yihui Xie, and Jonathan McPherson (2018). shiny: Web Application Framework for R. R package version 1.1.0. eprint: https : //CRAN . Rproject.org/package=shiny (cit. on pp. 85, 110).

Chen, Xiaoli, Sünje Dallmeier-Tiessen, Anxhela Dani, et al. (2016). „CERN Analysis Preservation: A Novel Digital Library Service to Enable Reusable and Reproducible Research“. In: Research and Advanced Technology for Digital Libraries. Springer International Publishing, pp. 347-356. eprint: http://dx.doi.org/10.1007/978-3-319-43997-6_45 (cit. on p. 62).

Chirigati, Fernando, Rémi Rampin, Dennis Shasha, and Juliana Freire (2016). „ReproZip: Computational Reproducibility With Ease“. In: Proceedings of the 2016 International Conference on Management of Data. SIGMOD '16. ACM, pp. 2085-2088. eprint: http: //doi.acm.org/10.1145/2882903.2899401 (cit. on p. 108). 
Chirigati, Fernando, Dennis Shasha, and Juliana Freire (2013). „ReproZip: Using Provenance to Support Computational Reproducibility“. In: Presented as part of the 5th USENIX Workshop on the Theory and Practice of Provenance. USENIX. eprint: https://www . usenix. org / conference / tapp13 / reprozip - using - provenance - support - computational reproducibilitythe (cit. on p. 66).

Claerbout, Jon F. and Martin Karrenbach (1992). „Electronic documents give reproducible research a new meaning". In: SEG Technical Program Expanded Abstracts 1992. Society of Exploration Geophysicists, pp. 601-604. eprint: https://doi.org/10.1190/1.1822162 (cit. on pp. 3, 42, 84, 102).

Clarkson, Chris, Mike Smith, Ben Marwick, et al. (2015). „The archaeology, chronology and stratigraphy of Madjedbebe (Malakunanja II): A site in northern Australia with early occupation“. In: Journal of Human Evolution 83, pp. 46-64. eprint: https : //doi .org/ 10.1016/j.jhevol.2015.03.014 (cit. on p. 66).

Cleveland, William S. (1984). „Graphs in Scientific Publications“. In: The American Statistician 38.4, pp. 261-269. eprint: https://doi.org/10.1080/00031305.1984.10483223 (cit. on p. 84).

Collberg, Christian and Todd A. Proebsting (2016). „Repeatability in Computer Systems Research“. In: Commun. ACM 59.3, pp. 62-69. eprint: http://doi.acm.org/10.1145/ 2812803 (cit. on pp. 2, 15, 20, 40, 41).

Colomb, Julien and Björn Brembs (2014). „Sub-strains of Drosophila Canton-S differ markedly in their locomotor behavior". In: F1000Research 3, p. 176. eprint: https: //dx.doi.org/10.12688/f1000research. 4263.1 (cit. on p. 110).

Conn, Vicki S., Sang-arun Isaramalai, Sabyasachi Rath, et al. (2004). „Beyond MEDLINE for Literature Searches“. In: Journal of Nursing Scholarship 35.2, pp. 177-182. eprint: https://doi.org/10.1111/j.1547-5069.2003.00177.x (cit. on p. 83).

Costello, Mark J. (2009). „Motivating Online Publication of Data“. In: BioScience 59.5, pp. 418-427. eprint: https://doi.org/10.1525/bio.2009.59.5.9 (cit. on p. 40).

Darch, Peter T. and Emily J.M. Knox (2017). „Ethical perspectives on data and software sharing in the sciences: A research agenda". In: Library Information Science Research 39.4, pp. 295-302. eprint: https://doi.org/10.1016/j.lisr.2017.11.008 (cit. on pp. 41, 128).

Delory, Benjamin M, Mao Li, Christopher N Topp, and Guillaume Lobet (2018). ,archiDART v3. 0: A new data analysis pipeline allowing the topological analysis of plant root systems". In: F1000Research 7, p. 22. eprint: https://dx. doi .org/10.12688/f1000research. 13541.1 (cit. on p. 110).

DiBiase, David (1990). „Visualization in the earth sciences“. In: Earth and Mineral Sciences 59.2, pp. 13-18 (cit. on p. 85).

DiBiase, David, Alan M MacEachren, John B Krygier, and Catherine Reeves (1992). „Animation and the role of map design in scientific visualization". In: Cartography and geographic information systems 19.4, pp. 201-214. eprint: https://doi.org/10.1559/ 152304092783721295 (cit. on pp. 84, 85). 
Donoho, David L, Arian Maleki, Inam Ur Rahman, Morteza Shahram, and Victoria Stodden (2009). „Reproducible research in computational harmonic analysis“. In: Computing in Science \& Engineering 11.1. eprint: https://doi.org/10.1109/MCSE.2009.15 (cit. on pp. 40, 84).

Dottori, Francesco, Rui Figueiredo, Mario LV Martina, Daniela Molinari, A Scorzini, et al. (2016). „INSYDE: a synthetic, probabilistic flood damage model based on explicit cost analysis“. In: Natural Hazards and Earth System Sciences 16, pp. 2577-2591. eprint: https://doi.org/10.5194/nhess-16-2577-2016 (cit. on p. 115).

Dragicevic, Pierre, Yvonne Jansen, Abhraneel Sarma, Matthew Kay, and Fanny Chevalier (2019). „Increasing the Transparency of Research Papers with Explorable Multiverse Analyses". In: CHI 2019 - The ACM CHI Conference on Human Factors in Computing Systems. ACM. eprint: https://hal.inria.fr/hal-01976951/ (cit. on p. 134).

Drees, Bastian, Angelina Kraft, and Thomas Koprucki (2018). „Reproducible and comprehensible research results through persistently linked and visualized numerical simulation data“. In: Optical and Quantum Electronics 50.2, p. 59. eprint: https ://doi .org/10. 1007/s11082-018-1327-1 (cit. on p. 109).

Dua, Rajdeep, Reddy Raja, and Dharmesh Kakadia (2014). „Virtualization vs Containerization to Support PaaS“. In: 2014 IEEE International Conference on Cloud Engineering, pp. 610614. eprint: https://doi.org/10.1109/IC2E. 2014.41 (cit. on p. 66).

Easterbrook, Steve M (2014). „Open code for open science?“ In: Nature Geoscience 7.11, p. 779. eprint: https://doi .org/10.1038/ngeo2283 (cit. on pp. 2, 39, 41, 64, 67, 107).

Echtler, Florian and Maximilian Häußler (2018). „Open Source, Open Science, and the Replication Crisis in HCI". In: Extended Abstracts of the $2018 \mathrm{CHI}$ Conference on Human Factors in Computing Systems. ACM, alt02. eprint: https ://doi .org/10.1145/3170427. 3188395 (cit. on p. 107).

European Commission (2015). „Access to and preservation of scientific information in Europe". In: Report on the implementation of Commission Recommendation C(2012) 4890 final. eprint: https://doi .org/10.2777/975917 (cit. on p. 64).

Faniel, Ixchel M and Trond E Jacobsen (2010). „Reusing scientific data: How earthquake engineering researchers assess the reusability of colleagues' data". In: Computer Supported Cooperative Work (CSCW) 19.3-4, pp. 355-375. eprint: https://doi.org/10.1007/ s10606-010-9117-8 (cit. on p. 83).

Fehr, Jörg, Jan Heiland, Christian Himpe, and Jens Saak (2016). „Best practices for replicability, reproducibility and reusability of computer-based experiments exemplified by model reduction software". In: arXiv preprint arXiv:1607.01191. eprint: https://dx. doi . org/10.3934/Math.2016.3.261 (cit. on pp. 42, 59).

Ferreira, Catarina, Guillaume Bastille-Rousseau, Amanda M. Bennett, et al. (2015). „The evolution of peer review as a basis for scientific publication: directional selection towards a robust discipline?" In: Biological Reviews 91.3, pp. 597-610. eprint: https ://doi .org/ 10.1111/brv. 12185 (cit. on p. 22).

Franzblau, Lauren E and Kevin C Chung (2012). „Graphs, tables, and figures in scientific publications: the good, the bad, and how not to be the latter". In: The Journal of hand surgery 37.3, pp. 591-596. eprint: https ://doi .org/10.1016/j . jhsa.2011.12.041 (cit. on p. 86). 
Gentleman, Robert and Duncan Temple Lang (2007). „Statistical Analyses and Reproducible Research". In: Journal of Computational and Graphical Statistics 16.1, pp. 1-23. eprint: https://doi.org/10.1198/106186007x178663 (cit. on pp. 2, 20, 39, 40, 64, 76, 108, 127, 129).

Gertler, Paul, Sebastian Galiani, and Mauricio Romero (2018). „How to make replication the norm“. In: Nature 554.7693, pp. 417-419. eprint: https://doi .org/10.1038/d41586018-02108-9 (cit. on p. 38).

Gewin, Virginia (2016). „Data sharing: An open mind on open data“. In: Nature 529.7584, pp. 117-119. eprint: https ://doi .org/10.1038/nj7584-117a (cit. on pp. 2, 17, 19, 40-42, 84, 107).

Gil, Yolanda, Cédric H. David, Ibrahim Demir, et al. (2016). „Toward the Geoscience Paper of the Future: Best practices for documenting and sharing research from data to software to provenance". In: Earth and Space Science 3.10, pp. 388-415. eprint: https://doi . org/10.1002/2015EA000136 (cit. on pp. 1, 17, 19, 20, 38, 40, 42, 84, 108).

Giraud, Timothée and Nicolas Lambert (2017). „Reproducible Cartography“. In: Advances in Cartography and GIScience. Springer International Publishing, pp. 173-183. eprint: https://doi.org/10.1007/978-3-319-57336-6_13 (cit. on pp. 3, 15, 38, 42, 49, 82, 84, 102).

Glandon, Phillip (2011). „Appendix to the Report of the Editor: Report on the American Economic Review Data Availability Compliance Project“. In: American Economic Review 101.3, pp. 695-699. eprint: https://doi.org/10.1257/aer.101.3.684 (cit. on p. 67).

Glaser, Barney and Anselm Strauss (1967). „Grounded theory: The discovery of grounded theory“. In: Sociology the journal of the British sociological association 12, pp. 27-49 (cit. on pp. 7, 44, 47, 86).

Goecks, Jeremy, Anton Nekrutenko, James Taylor, and The Galaxy Team (2010). „Galaxy: a comprehensive approach for supporting accessible, reproducible, and transparent computational research in the life sciences“. In: Genome Biology 11.8, R86. eprint: https: //doi.org/10.1186/gb-2010-11-8-r86 (cit. on pp. 62, 110).

Goodchild, Michael F. (1992). „Geographical information science“. In: International Journal of Geographical Information Systems 6.1, pp. 31-45. eprint: https ://doi .org/10.1080/ 02693799208901893 (cit. on pp. 1, 38).

Goodman, Steven N., Daniele Fanelli, and John P. A. Ioannidis (2016). „What does research reproducibility mean?" In: Science Translational Medicine 8.341, 341ps12-341ps12. eprint: https://doi.org/10.1126/scitranslmed.aaf5027 (cit. on pp. 2, 8, 38, 39, 63, 83, 107).

Greenbaum, Dov, Joel Rozowsky, Victoria Stodden, and Mark Gerstein (2017). „Structuring supplemental materials in support of reproducibility". In: Genome Biology 18.1, p. 64. eprint: https://doi.org/10.1186/s13059-017-1205-3 (cit. on p. 32).

Greis, Miriam, Hendrik Schuff, Marius Kleiner, Niels Henze, and Albrecht Schmidt (2017). „Input Controls for Entering Uncertain Data: Probability Distribution Sliders“. In: Proceedings of the ACM on Human-Computer Interaction 1.1, p. 3. eprint: https://doi .org/10. 1145/3095805 (cit. on p. 110). 
Gronenschild, Ed H. B. M., Petra Habets, Heidi I. L. Jacobs, et al. (2012). „The Effects of FreeSurfer Version, Workstation Type, and Macintosh Operating System Version on Anatomical Volume and Cortical Thickness Measurements“. In: PLOS ONE 7.6, pp. 1-13. eprint: https://doi.org/10.1371/journal.pone.0038234 (cit. on p. 19).

Haak, Laurel L., Martin Fenner, Laura Paglione, Ed Pentz, and Howard Ratner (2012). "ORCID: a system to uniquely identify researchers". In: Learned Publishing 25.4, pp. 259264. eprint: https://doi.org/10.1087/20120404 (cit. on p. 68).

Hanson, Karen L., Tim DiLauro, and Mark Donoghue (2015). „The RMap Project: Capturing and Preserving Associations Amongst Multi-Part Distributed Publications“. In: Proceedings of the 15th ACM/IEEE-CS Joint Conference on Digital Libraries. JCDL '15. ACM, pp. 281282. eprint: http://doi.acm.org/10.1145/2756406.2756952 (cit. on p. 62).

Harnad, Stevan, Tim Brody, François Vallières, et al. (2004). „The Access/Impact Problem and the Green and Gold Roads to Open Access“. In: Serials Review 30.4, pp. 310-314. eprint: https://doi.org/10.1016/j. serrev. 2004.09.013 (cit. on pp. 63, 107).

Hearst, Marti (2006). „Design recommendations for hierarchical faceted search interfaces“. In: ACM SIGIR workshop on faceted search. Seattle, WA, pp. 1-5 (cit. on p. 83).

Hegarty, Mary, Harvey S Smallman, Andrew T Stull, and Matt S Canham (2009). „Naive cartography: How intuitions about display configuration can hurt performance“. In: Cartographica: The International Journal for Geographic Information and Geovisualization 44.3, pp. 171-186. eprint: https://doi.org/10.3138/carto.44.3.171 (cit. on p. 85).

Heiberger, Richard and Naomi Robbins (2014). „Design of Diverging Stacked Bar Charts for Likert Scales and Other Applications“. In: Journal of Statistical Software, Articles 57.5, pp. 1-32. eprint: http://dx.doi.org/10.18637/jss.v057.i05 (cit. on pp. 44, 46).

Herndon, Thomas, Michael Ash, and Robert Pollin (2014). „Does high public debt consistently stifle economic growth? A critique of Reinhart and Rogoff". In: Cambridge Journal of Economics 38.2, pp. 257-279. eprint: http://dx.doi.org/10.1093/cje/bet075 (cit. on pp. 75,128$)$.

Hillebrand, Helmut and Jessica Gurevitch (2013). „Reporting standards in experimental studies“. In: Ecology Letters 16.12, pp. 1419-1420. eprint: https://doi .org/10.1111/ ele.12190 (cit. on p. 40).

Hoppe, Anett, Jascha Hagen, Helge Holzmann, Günter Kniesel, and Ralph Ewerth (2018). "An Analytics Tool for Exploring Scientific Software and Related Publications“. In: Digital Libraries for Open Knowledge. Ed. by Eva Méndez, Fabio Crestani, Cristina Ribeiro, Gabriel David, and João Correia Lopes. Springer International Publishing, pp. 299-303. eprint: https://doi.org/10.1007/978-3-030-00066-0_27 (cit. on p. 109).

Hothorn, Torsten and Friedrich Leisch (2011). „Case studies in reproducibility“. In: Briefings in Bioinformatics 12.3, pp. 288-300. eprint: http://dx.doi.org/10.1093/bib/bbq084 (cit. on p. 15).

Howe, Bill (2012). „Virtual appliances, cloud computing, and reproducible research“. In: Computing in Science \& Engineering 14.4, pp. 36-41. eprint: https : // doi . org/10 . 1109/mcse. 2012.62 (cit. on pp. 66, 77). 
Hung, Ling-Hong, Daniel Kristiyanto, Sung Bong Lee, and Ka Yee Yeung (2016). „GUIdock: Using Docker Containers with a Common Graphics User Interface to Address the Reproducibility of Research“. In: PLOS ONE 11.4, pp. 1-14. eprint: https : // doi .org/10. 1371/journal pone.0152686 (cit. on p. 66).

Ifenthaler, Dirk (2008). „Relational, structural, and semantic analysis of graphical representations and concept maps". In: Educational Technology Research and Development 58.1, pp. 81-97. eprint: https://doi.org/10.1007/s11423-008-9087-4 (cit. on p. 142).

Ince, Darrel C., Leslie Hatton, and John Graham-Cumming (2012). „The case for open computer programs“. In: Nature 482.7386, pp. 485-488. eprint: https : //doi .org/10 1038/nature10836 (cit. on p. 31).

Ioannidis, John P. A. (2005). „Why Most Published Research Findings Are False“. In: PLOS Medicine 2.8, e124. eprint: https://doi.org/10.1371/journal.pmed.0020124 (cit. on p. 13).

- (2014). „How to Make More Published Research True“. In: PLOS Medicine 11.10, e1001747. eprint: https://doi.org/10.1371/journal.pmed.1001747 (cit. on p. 15).

Ioannidis, John P. A., David B Allison, Catherine A Ball, et al. (2009). „Repeatability of published microarray gene expression analyses“. In: Nature genetics 41.2, pp. 149-155. eprint: https://doi.org/10.1038/ng.295 (cit. on pp. 41, 84).

Ioannidis, John P. A., T. D. Stanley, and Hristos Doucouliagos (2017). „The Power of Bias in Economics Research“. In: The Economic Journal 127.605, F236-F265. eprint: https://doi.org/10.1111/ecoj.12461 (cit. on p. 13).

Iqbal, Shareen A., Joshua D. Wallach, Muin J. Khoury, Sheri D. Schully, and John P. A. Ioannidis (2016). „Reproducible Research Practices and Transparency across the Biomedical Literature“. In: PLOS Biology 14.1, e1002333. eprint: https : / / doi .org/10 . 1371/ journal.pbio.1002333 (cit. on p. 2).

Jones, Nicola (2016). „AI science search engines expand their reach“. In: Nature. eprint: https://doi.org/10.1038/nature.2016.20964 (cit. on p. 34).

Kauppinen, Tomi and Giovana Mira de Espindola (2011). „Linked Open Science-Communicating, Sharing and Evaluating Data, Methods and Results for Executable Papers“. In: vol. 4. Proceedings of the International Conference on Computational Science, ICCS 2011, pp. 726731. eprint: https://doi.org/10.1016/j.procs.2011.04.076 (cit. on p. 109).

Keshav, Srinivasan (2007). „How to read a paper“. In: ACM SIGCOMM Computer Communication Review 37.3, pp. 83-84. eprint: https://doi.org/10.1145/1273445.1273458 (cit. on p. 91).

Kidwell, Mallory C., Ljiljana B. Lazarević, Erica Baranski, et al. (2016). „Badges to Acknowledge Open Practices: A Simple, Low-Cost, Effective Method for Increasing Transparency“. In: PLOS Biology 14.5, pp. 1-15. eprint: https : //doi .org/10.1371/journal . pbio. 1002456 (cit. on p. 32).

Kircz, Joost G. (1998). „Modularity: the next form of scientific information presentation?" In: Journal of documentation 54.2, pp. 210-235. eprint: https://doi.org/10.1108/ EUM0000000007185 (cit. on p. 83). 
Kluyver, Thomas, Benjamin Ragan-Kelley, Fernando Pérez, et al. (2016). „Jupyter Notebooksa publishing format for reproducible computational workflows". In: ELPUB, pp. 87-90. eprint: https://doi.org/10.3233/978-1-61499-649-1-87 (cit. on pp. 42, 59, 85, 108).

Knuth, Donald Ervin (1984). „Literate programming“. In: The Computer Journal 27.2, pp. 97-111. eprint: https://doi .org/10.1093/comjnl/27.2.97 (cit. on pp. 42, 64, 70, 108).

Kray, Christian, Edzer Pebesma, Markus Konkol, and Daniel Nüst (2019). „Reproducible Research in Geoinformatics: Concepts, Challenges and Benefits (in press.)" In: 14th International Conference on Spatial Information Theory (COSIT 2019) (cit. on pp. 138, 143).

Kules, Bill and Ben Shneiderman (2008). „Users can change their web search tactics: Design guidelines for categorized overviews". In: Information Processing \& Management 44.2, pp. 463-484. eprint: https://doi.org/10.1016/j.ipm.2007.07.014 (cit. on p. 83).

Kunze, J, J Littman, L Madden, et al. (2011). „The bagit file packaging format (v0. 97)“. In: eprint: https://tools.ietf.org/html/draft-kunze-bagit-13 (cit. on p. 74).

Kwakkel, Jan H, Stephen Carley, John Chase, and Scott W Cunningham (2014). „Visualizing geo-spatial data in science, technology and innovation". In: Technological Forecasting and Social Change 81, pp. 67-81. eprint: https://doi .org/10.1016/j.techfore.2012.09. 007 (cit. on pp. 84, 85).

Langheinrich, Marc, Albrecht Schmidt, Nigel Davies, and Rui José (2013). „A Practical Framework for Ethics: The PD-net Approach to Supporting Ethics Compliance in Public Display Studies“. In: Proceedings of the 2Nd ACM International Symposium on Pervasive Displays. PerDis '13. ACM, pp. 139-143. eprint: http://doi . acm . org/10.1145/2491568. 2491598 (cit. on p. 138).

Lazar, Jonathan, Jinjuan Heidi Feng, and Harry Hochheiser (2017). Research methods in human-computer interaction. Morgan Kaufmann (cit. on pp. 6, 7, 44, 85, 86).

Lee, Po-shen, Jevin D West, and Bill Howe (2017). „Viziometrics: Analyzing Visual Information in the Scientific Literature“. In: IEEE Transactions on Big Data 4.1, pp. 117-129. eprint: https://doi.org/10.1109/TBDATA.2017.2689038 (cit. on p. 84).

Leek, Jeffrey T. and Leah R. Jager (2017). „Is Most Published Research Really False?“ In: Annual Review of Statistics and Its Application 4.1, pp. 109-122. eprint: https : //doi .org/10 .1146/annurev-statistics-060116-054104 (cit. on pp. 38, 40, 56, 130).

Leek, Jeffrey T. and Roger D. Peng (2015). „Opinion: Reproducible research can still be wrong: Adopting a prevention approach". In: Proceedings of the National Academy of Sciences 112.6, pp. 1645-1646. eprint: https://doi.org/10.1073/pnas. 1421412111 (cit. on pp. 16, 39, 63, 83).

Leisch, Friedrich (2002). „Sweave: Dynamic generation of statistical reports using literate data analysis“. In: Compstat. Springer, pp. 575-580. eprint: https://doi.org/10.1007/ 978-3-642-57489-4_89 (cit. on p. 108).

Lindgaard, Gitte and Jarinee Chattratichart (2007). „Usability testing“. In: Proceedings of the SIGCHI conference on Human factors in computing systems - CHI '07. ACM Press. eprint: https://doi.org/10.1145/1240624.1240839 (cit. on p. 135). 
Lovelace, Robin, Jakub Nowosad, and Jannes Muenchow (2016). „Geocomputation with R“. In: eprint: https://geocompr.robinlovelace.net/ (cit. on p. 42).

MacEachren, Alan M and Menno-Jan Kraak (1997). Exploratory cartographic visualization: advancing the agenda. eprint: https ://doi .org/10 .1016/S0098-3004(97) 00018-6 (cit. on p. 85).

Mack, Chris A. (2014). „How to write a good scientific paper: figures, part 2“. In: Journal of Micro/Nanolithography, MEMS, and MOEMS 13.1, p. 010102. eprint: https://doi.org/ 10.1117/1. JMM.13.1.010102 (cit. on p. 84).

El-Madany, Tarek S., J. B. Walk, M. J. Deventer, et al. (2016). „Canopy-atmosphere interactions under foggy condition-Size-resolved fog droplet fluxes and their implications". In: Journal of Geophysical Research: Biogeosciences 121.3, pp. 796-808. eprint: https: //doi.org/10.1002/2015JG003221 (cit. on p. 90).

Markowetz, Florian (2015). „Five selfish reasons to work reproducibly“. In: Genome Biology 16.1, p. 274. eprint: https://doi .org/10.1186/s13059-015-0850-7 (cit. on pp. 32, 56, 67, 102).

Marlon, Jennifer R., R. Kelly, A.-L. Daniau, et al. (2016). „Reconstructions of biomass burning from sediment-charcoal records to improve data-model comparisons". In: Biogeosciences 13.11, pp. 3225-3244. eprint: https ://doi .org/10.5194/bg-13-3225-2016 (cit. on pp. 53-55, 60).

Marwick, Ben (2015). 1989-Excavation-Report-Madjebebe. eprint: https://doi .org/10. 6084/m9.figshare.1297059.v1 (cit. on p. 66).

Matarese, Valerie (2006). „Introductory Course on Getting to Know Journals and on "Browsing" a Research Paper: First Steps to Proficiency in Scientific Communication“. In: Croatian medical journal 47.5, pp. 767-775. eprint: https : //www.ncbi.nlm.nih.gov/pmc/ articles/PMC2080472/ (cit. on p. 83).

McCullough, B.D., Kerry Anne McGeary, and Teresa D. Harrison (2008). „Do economics journal archives promote replicable research?" In: Canadian Journal of Economics/Revue canadienne d'économique 41.4, pp. 1406-1420. eprint: https://doi.org/10.1111/j. 1540-5982.2008.00509.x (cit. on p. 41).

McKiernan, Erin C. (2017). „Imagining the "open” university: Sharing scholarship to improve research and education". In: PLOS Biology 15.10, pp. 1-25. eprint: https ://doi .org/ 10.1371/journal.pbio.1002614 (cit. on p. 31).

McKiernan, Erin C., Philip E Bourne, C Titus Brown, et al. (2016). „Point of View: How open science helps researchers succeed“. In: eLife 5, e16800. eprint: https://doi .org/10 . 7554/eLife. 16800 (cit. on p. 35).

McNutt, Marcia (2014). Journals unite for reproducibility. eprint: https ://doi .org/10 . 1126/science . aaa1724 (cit. on pp. 15, 17, 19).

Mease, Jon (2018). „Bringing ipywidgets Support to plotly.py“. In: Proceedings of the 17th Python in Science Conference. SciPy. eprint: https : / doi .org/10 .25080/majora4af1f417-00b (cit. on p. 110).

Mecklenburg, Robert (2004). Managing Projects with GNU Make: The Power of GNU Make for Building Anything, 3rd Edition. O'Reilly Media, Inc. (cit. on p. 65). 
Merali, Zeeya (2010). „Computational science: Error, why scientific programming does not compute“. In: Nature 467.7317, pp. 775-777. eprint: http://dx. doi .org/10.1038/ 467775a (cit. on p. 84).

Merkel, Dirk (2014). „Docker: Lightweight Linux Containers for Consistent Development and Deployment“. In: Linux J. 2014.239. eprint: http://dl . acm . org/citation. cfm? $i d=2600239.2600241$ (cit. on p. 66).

Monge, Alvaro E. and Charles P. Elkan (1996). „The webfind tool for finding scientific papers over the Worldwide Web". In: Proceedings of the 3rd International Congress on Computer Science Research, pp. 41-46 (cit. on p. 83).

Murray, Daniel G. (2013). Tableau your data!: fast and easy visual analysis with tableau software. John Wiley \& Sons (cit. on p. 101).

Narayanan, Arvind, Joanna Huey, and Edward W. Felten (2016). „A Precautionary Approach to Big Data Privacy“. In: Data Protection on the Move. Springer Netherlands, pp. 357-385. eprint: https://doi.org/10.1007/978-94-017-7376-8_13 (cit. on p. 128).

Nature Editorial (2014). „Code share“. In: Nature 514.7524, p. 536. eprint: https ://doi. org/10.1038/514536a (cit. on p. 64).

- (2016). „Reality check on reproducibility“. In: Nature 533.7604, pp. 437-437. eprint: https://doi.org/10.1038/533437a (cit. on pp. 15, 16).

Nielsen, Jakob, James Lewis, and Carl Turner (2006). „Determining Usability Test Sample Size“. In: International Encyclopedia of Ergonomics and Human Factors, Second Edition - 3 Volume Set. CRC Press. eprint: https://doi .org/10.1201/9780849375477. ch597 (cit. on p. 135).

Nosek, Brian A., G. Alter, G. C. Banks, et al. (2015). „Promoting an open research culture“. In: Science 348.6242, pp. 1422-1425. eprint: https : //doi .org/10.1126/science. aab2374 (cit. on pp. 2, 17, 19, 41, 61, 84, 94).

Ooms, Jeroen (2018). opencpu. Accessed: 2018-10-01. eprint: https://CRAN.R-project . org/package=opencpu (cit. on p. 110).

Open Science Collaboration (2015). „Estimating the reproducibility of psychological science“. In: Science 349.6251, aac4716. eprint: https ://doi .org/10.1126/science . aac4716 (cit. on p. 41).

Ostermann, Frank O. and Carlos Granell (2017). „Advancing Science with VGI: Reproducibility and Replicability of Recent Studies using VGI“. In: Transactions in GIS 21.2, pp. 224 237. eprint: https://doi.org/10.1111/tgis.12195 (cit. on pp. 2, 15, 16, 41, 42, 83, 125).

Pain, Elisabeth (2016). How to (seriously) read a scientific paper. eprint: https ://doi .org/ 10.1126/science. caredit.a1600047 (cit. on p. 83).

Palma, Raúl, Oscar Corcho, Piotr Hotubowicz, et al. (2013). „Digital Libraries for the Preservation of Research Methods and Associated Artifacts“. In: Proceedings of the 1st International Workshop on Digital Preservation of Research Methods and Artefacts. DPRMA '13. Indianapolis, Indiana, USA: ACM, pp. 8-15. eprint: http://doi.acm.org/10.1145/ 2499583. 2499589 (cit. on p. 64). 
Patil, Prasad, Roger D. Peng, and Jeffrey Leek (2016). „A statistical definition for reproducibility and replicability“. In: bioRxiv. eprint: https : //doi .org/10.1101/066803 (cit. on p. 83).

Pebesma, Edzer (2012). „spacetime: Spatio-Temporal Data in R“. In: Journal of Statistical Software 51.7, pp. 1-30. eprint: https://doi.org/10.18637/jss.v051.i07 (cit. on p. 98).

- (2013). Earth and Planetary Innovation Challenge (EPIC) Submission "One-Click-Reproduce". Accessed: 2019-01-16. eprint: http : // pebesma. staff .ifgi.de/epic.pdf (cit. on pp. 10, 68, 129).

Peng, Roger D. (2011). „Reproducible Research in Computational Science“. In: Science 334.6060, pp. 1226-1227. eprint: https://doi.org/10.1126/science.1213847 (cit. on pp. $32,40,127)$.

Perin, Charles, Frédéric Vernier, and Jean-Daniel Fekete (2013). „Interactive Horizon Graphs: Improving the Compact Visualization of Multiple Time Series“. In: Proceedings of the SIGCHI Conference on Human Factors in Computing Systems. CHI '13. ACM, pp. 32173226. eprint: http://doi .acm.org/10.1145/2470654.2466441 (cit. on p. 85).

Perkel, Jeffrey M. (2018). „Data visualization tools drive interactivity and reproducibility in online publishing“. In: Nature 554.7690, pp. 133-134. eprint: https://doi .org/10. 1038/d41586-018-01322-9 (cit. on p. 110).

Pham, Quan, Tanu Malik, and Ian T. Foster (2013). „Using Provenance for Repeatability“. In: TaPP 13, p. 2. eprint: https : / www . usenix . org/ conference/tapp13/using provenance-repeatability (cit. on pp. 119, 124).

Pham, Quan, Tanu Malik, Ian Foster, Roberto Di Lauro, and Raffaele Montella (2012). „SOLE: linking research papers with science objects". In: International Provenance and Annotation Workshop. Springer, pp. 203-208. eprint: https://doi .org/10.1007/9783-642-34222-6_16 (cit. on pp. 2, 83, 108, 109).

Piwowar, Heather A., Roger S. Day, and Douglas B. Fridsma (2007). „Sharing Detailed Research Data Is Associated with Increased Citation Rate“. In: PLOS ONE 2.3, pp. 1-5. eprint: https://doi.org/10.1371/journal. pone. 0000308 (cit. on pp. 40, 41, 84).

Powers, Stephen M. and Stephanie E. Hampton (2019). „Open science, reproducibility, and transparency in ecology“. In: Ecological Applications 29.1, e01822. eprint: https: // esajournals . onlinelibrary. wiley. com/doi/pdf / 10.1002/eap. 1822 (cit. on p. 128).

Pundt, Hardy and Fred Toppen (2017). „20 Years of AGILE“. In: Societal Geo-innovation. Ed. by Arnold Bregt, Tapani Sarjakoski, Ron van Lammeren, and Frans Rip. Springer International Publishing, pp. 351-367. eprint: https ://doi.org/10 .1007/978-3-31956759-4_20 (cit. on p. 16).

QGIS Development Team (2018). QGIS Geographic Information System. Open Source Geospa-tial Foundation Project. (Cit. on p. 101).

R Core Team (2018). R: A Language and Environment for Statistical Computing. R Foundation for Statistical Computing. eprint: http://www.R-project.org/ (cit. on pp. 4, 49, $66,85,107)$. 
Radke, Richard J., Srinivas Andra, Omar Al-Kofahi, and Badrinath Roysam (2005). „Image change detection algorithms: a systematic survey“. In: IEEE transactions on image processing 14.3, pp. 294-307. eprint: https://doi.org/10.1109/TIP. 2004.838698 (cit. on p. 123).

Rathfux, Thomas, Roman Popp, and Hermann Kaindl (2016). „Adding custom widgets to model-driven GUI generation". In: Proceedings of the 8th ACM SIGCHI Symposium on Engineering Interactive Computing Systems. ACM, pp. 16-26. eprint: https ://doi .org/ 10.1145/2933242.2933251 (cit. on p. 106).

Rawat, Seema and Sanjay Meena (2014). „Publish or perish: Where are we heading?“ In: Journal of research in medical sciences: the official journal of Isfahan University of Medical Sciences 19.2, p. 87. eprint: https://www.ncbi.nlm.nih.gov/pmc/articles/ PMC3999612/ (cit. on p. 138).

Reichman, O. J., Matthew B. Jones, and Mark P. Schildhauer (2011). „Challenges and Opportunities of Open Data in Ecology“. In: Science 331.6018, pp. 703-705. eprint: https://doi.org/10.1126/science.1197962 (cit. on pp. 2, 19, 38, 40, 41, 84).

Reinhart, Carmen M. and Kenneth S. Rogoff (2010). „Growth in a Time of Debt“. In: American Economic Review 100.2, pp. 573-78. eprint: https://doi .org/10.1257/aer.100.2.573 (cit. on p. 75)

Renear, Allen H. and Carole L. Palmer (2009). „Strategic reading, ontologies, and the future of scientific publishing“. In: Science 325.5942, pp. 828-832. eprint: https://doi .org/ 10.1126/science.1157784 (cit. on p. 83).

Rolandi, Marco, Karen Cheng, and Sarah Pérez-Kriz (2011). „A brief guide to designing effective figures for the scientific paper". In: Advanced Materials 23.38, pp. 4343-4346. eprint: https://doi.org/10.1002/adma.201102518 (cit. on p. 84).

RStudio Team (2015). RStudio: Integrated Development Environment for R. RStudio, Inc. eprint: http://www.rstudio.com/ (cit. on p. 50).

Sandusky, Robert J. and Carol Tenopir (2008). „Finding and using journal-article components: Impacts of disaggregation on teaching and research practice". In: Journal of the American Society for Information Science and Technology 59.6, pp. 970-982. eprint: https://doi org/10.1002/asi. 20804 (cit. on p. 83).

Sandusky, Robert J., Carol Tenopir, and Margaret M. Casado (2007). „Figure and table retrieval from scholarly journal articles: User needs for teaching and research". In: Proceedings of the American Society for Information Science and Technology 44.1, pp. 1-13. eprint: https://doi.org/10.1002/meet.1450440390 (cit. on p. 84).

Sandve, Geir Kjetil, Anton Nekrutenko, James Taylor, and Eivind Hovig (2013). „Ten Simple Rules for Reproducible Computational Research“. In: PLOS Computational Biology 9.10, pp. 1-4. eprint: https://doi.org/10.1371/journal.pcbi.1003285 (cit. on pp. 17, 19, $20,42,59,114)$.

Santana-Perez, Idafen, Rafael Ferreira da Silva, Mats Rynge, et al. (2017). „Reproducibility of execution environments in computational science using Semantics and Clouds“. In: Future Generation Computer Systems 67, pp. 354-367. eprint: https ://doi .org/10 . 1016/j.future.2015.12.017 (cit. on p. 66). 
Savage, Caroline J. and Andrew J. Vickers (2009). „Empirical study of data sharing by authors publishing in PLoS journals". In: PloS one 4.9, e7078. eprint: https://doi .org/ 10.1371/journal. pone. 0007078 (cit. on pp. 82, 84).

Scheider, Simon, Frank O. Ostermann, and Benjamin Adams (2017). „Why good data analysts need to be critical synthesists. Determining the role of semantics in data analysis". In: Future generation computer systems 72, pp. 11-22. eprint: https://doi.org/10.1016/ j.future.2017.02.046 (cit. on p. 17).

Sievert, Carson, Chris Parmer, Toby Hocking, et al. (2017). plotly: Create Interactive Web Graphics via 'plotly. js'. R package version 4.7. 1 (cit. on p. 110).

Singh Chawla, Dalmeet (2015). „Living figures' make their debut“. In: Nature News 521.7550, p. 112. eprint: https : / doi.org/10.1038/nature.2015.17382 (cit. on pp. 3, 110).

Skaggs, T.H., M.H. Young, and J.A. Vrugt (2015). „Reproducible Research in Vadose Zone Sciences“. In: Vadose Zone Journal 14.10, vzj2015.06.0088. eprint: http://dx . doi .org/ 10.2136/vzj2015.06.0088 (cit. on pp. 2, 43).

Steiniger, Stefan and Geoffrey J. Hay (2009). „Free and open source geographic information tools for landscape ecology“. In: Ecological Informatics 4.4, pp. 183-195. eprint: https: //doi.org/10.1016/j.ecoinf.2009.07.004 (cit. on pp. 19, 42, 107).

Stodden, Victoria (2010). „The Scientific Method in Practice: Reproducibility in the Computational Sciences“. In: MIT Sloan Research Paper 4773.10. eprint: http://dx. doi .org/ 10.2139/ssrn. 1550193 (cit. on pp. 38, 129).

Stodden, Victoria, Matthew S. Krafczyk, and Adhithya Bhaskar (2018). „Enabling the Verification of Computational Results: An Empirical Evaluation of Computational Reproducibility“. In: Proceedings of the First International Workshop on Practical Reproducible Evaluation of Computer Systems. ACM, p. 3. eprint: https://doi .org/10.1145/3214239.3214242 (cit. on p. 108).

Stodden, Victoria, Friedrich Leisch, and Roger D. Peng (2014). Implementing reproducible research. CRC Press (cit. on p. 67).

Stodden, Victoria, Marcia McNutt, David H. Bailey, et al. (2016). „Enhancing reproducibility for computational methods“. In: Science 354.6317, pp. 1240-1241. eprint: https: //doi . org/10.1126/science. aah6168 (cit. on pp. 2, 15-17, 19, 20, 40-42, 57, 82, 84, 124).

Stodden, Victoria and Sheila Miguez (2014). „Best Practices for Computational Science: Software Infrastructure and Environments for Reproducible and Extensible Research". In: Journal of Open Research Software 2 (1), p.e21. eprint: http://doi .org/10.5334/jors . ay (cit. on pp. 17, 20).

Stodden, Victoria, Sheila Miguez, and Jennifer Seiler (2015). „ResearchCompendia.org: Cyberinfrastructure for Reproducibility and Collaboration in Computational Science“. In: Computing in Science \& Engineering 17.1, pp. 12-19. eprint: https ://doi .org/10. 1109/MCSE. 2015.18 (cit. on p. 64).

Teal, Tracy K., Karen A. Cranston, Hilmar Lapp, et al. (2015). „Data carpentry: workshops to increase data literacy for researchers“. In: International Journal of Digital Curation 10.1, pp. 135-143. eprint: https://doi.org/10.2218/ijdc.v10i1.351 (cit. on p. 31). 
Tenopir, Carol, Suzie Allard, Kimberly Douglass, et al. (2011). „Data Sharing by Scientists: Practices and Perceptions“. In: PLOS ONE 6.6, pp. 1-21. eprint: https://doi.org/10. 1371/journal. pone.0021101 (cit. on p. 15).

Tenopir, Carol, Donald W King, Sheri Edwards, and Lei Wu (2009). „Electronic journals and changes in scholarly article seeking and reading patterns". In: Aslib proceedings. Vol. 61. 1. Emerald Group Publishing Limited, pp. 5-32. eprint: https : //doi .org/10.1108/ 00012530910932267 (cit. on p. 83).

Thain, Douglas, Peter Ivie, and Haiyan Meng (2015). „Techniques for preserving scientific software executions: Preserve the mess or encourage cleanliness?" In: Proceedings of the 12th International Conference on Digital Preservation (iPRES), pp. 164-174. eprint: https://doi.org/10.7274/ROCZ353M (cit. on p. 66).

That, D. H. T., G. Fils, Z. Yuan, and T. Malik (2017). „Sciunits: Reusable Research Objects“. In: 2017 IEEE 13th International Conference on e-Science (e-Science), pp. 374-383. eprint: https://doi.org/10.1109/eScience.2017.51 (cit. on p. 108).

Trestle Technology, LLC (2018). plumber: An API Generator for R. R package version 0.4.6. eprint: https ://CRAN.R-project.org/package=plumber (cit. on p. 110).

Tversky, Barbara, Julie Bauer Morrison, and Mireille Betrancourt (2002). „Animation: can it facilitate?" In: International journal of human-computer studies 57.4, pp. 247-262. eprint: https://doi.org/10.1006/ijhc.2002.1017 (cit. on p. 85).

Vaidyanathan, Ramnath, Yihui Xie, JJ Allaire, Joe Cheng, and Kenton Russell (2016). htmlwidgets: HTML Widgets for R. Accessed: 2018-10-01. eprint: https : / CRAN . Rproject.org/package=htmlwidgets (cit. on p. 110).

Vandewalle, Patrick (2012). „Code Sharing Is Associated with Research Impact in Image Processing“. In: Computing in Science \& Engineering 14.4, pp. 42-47. eprint: https : //doi.org/10.1109/MCSE. 2012.63 (cit. on pp. 2, 40, 84).

Vandewalle, Patrick, Jelena Kovacevic, and Martin Vetterli (2009). „Reproducible research in signal processing“. In: IEEE Signal Processing Magazine 26.3, pp. 37-47. eprint: https: //doi.org/10.1109/MSP.2009.932122 (cit. on pp. 41, 63).

Vines, Timothy H., Arianne YK. Albert, Rose L. Andrew, et al. (2014). „The availability of research data declines rapidly with article age“. In: Current biology 24.1, pp. 94-97. eprint: https://doi.org/10.1016/j.cub.2013.11.014 (cit. on pp. 41, 63).

Wacharamanotham, Chat, Krishna Subramanian, Sarah Theres Völkel, and Jan Borchers (2015). „Statsplorer: Guiding novices in statistical analysis“. In: Proceedings of the 33rd Annual ACM Conference on Human Factors in Computing Systems. ACM, pp. 2693-2702. eprint: https://doi.org/10.1145/2702123.2702347 (cit. on pp. 82, 84, 85, 110, 142).

Weissgerber, Tracey L., Vesna D. Garovic, Marko Savic, Stacey J. Winham, and Natasa M. Milic (2016). „From static to interactive: transforming data visualization to improve transparency“. In: PLoS biology 14.6, e1002484. eprint: https ://doi .org/10.1371/ journal.pbio. 1002545 (cit. on pp. 84, 85).

Welty, Leah J., L.V. Rasmussen, A.S. Baldridge, and E. Whitley (2016). StatTag. Galter Health Sciences Library. Chicago, Illinois, United States. eprint: https://doi.org/10.18131/ G36K76 (cit. on p. 108). 
Wilkinson, Mark D., Michel Dumontier, IJsbrand Jan Aalbersberg, et al. (2016). „The FAIR Guiding Principles for scientific data management and stewardship“. In: Scientific data 3. eprint: https://doi.org/10.1038/sdata.2016.18 (cit. on pp. 56, 109).

Wilson, Greg (2006). „Software Carpentry: Getting Scientists to Write Better Code by Making Them More Productive“. In: Computing in Science Engineering 8.6, pp. 66-69. eprint: https://doi.org/10.1109/mcse.2006.122 (cit. on p. 31).

Wilson, Greg, Jennifer Bryan, Karen Cranston, et al. (2017). „Good enough practices in scientific computing“. In: PLOS Computational Biology 13.6, pp. 1-20. eprint: https: //doi.org/10.1371/journal.pcbi.1005510 (cit. on pp. 32, 35).

Wolstencroft, Katherine, Robert Haines, Donal Fellows, et al. (2013). „The Taverna workflow suite: designing and executing workflows of Web Services on the desktop, web or in the cloud“. In: Nucleic acids research 41.W1, W557-W561. eprint: https ://doi .org/10 . 1093/nar/gkt328 (cit. on p. 65).

Wu, Yelena P., Brandon S. Aylward, Michael C. Roberts, and Spencer C. Evans (2012). "Searching the scientific literature: implications for quantitative and qualitative reviews". In: Clinical Psychology Review 32.6, pp. 553-557. eprint: https://doi.org/10.1016/j cpr.2012.06.007 (cit. on p. 83).

Yale Law School Roundtable on Data and Code Sharing (2010). „Reproducible Research“. In: Computing in Science Engineering 12.5, pp. 8-13. eprint: https://doi.org/10.1109/ MCSE. 2010.113 (cit. on p. 31).

\section{Websites}

Holdgraf, Chris (2017). Binder 2.0, a Tech Guide. URL: https : / / blog . jupyter . org / binder-2-0-a-tech-guide-2017-fd40515a3a84 (visited on Feb. 20, 2019) (cit. on p. 29).

Nature Geosciences (2018). Aims and Scope. URL: https://www . nature.com/ngeo/about/ aims (visited on Feb. 20, 2019) (cit. on pp. 3, 38).

Nüst, Daniel (2018). Reproducibility Package for "Reproducible research and GIScience: an evaluation using AGILE conference papers". URL: https://doi .org/10.5281/zenodo. 1227260 (visited on Feb. 20, 2019) (cit. on pp. 17, 27, 29).

Priem, Jason, Dario Taraborelli, Paul Goth, and Cameron Neylon (2017). altmetrics: a manifesto-altmetrics.org. URL: http://altmetrics .org/manifesto/ (visited on Feb. 20, 2019) (cit. on p. 31).

Rodriguez, Natalia (2015). Infographic: How to read a scientific paper. URL: https : //www. elsevier.com/connect/infographic-how-to-read-a-scientific-paper (visited on Feb. 20, 2019) (cit. on p. 83).

Victor, Bret (2018). Explorable Explanations. URL: http://worrydream . com/\#! /ExplorableExplanations (visited on Feb. 20, 2019) (cit. on pp. 110, 134).

Wongsuphasawat, Kanit, Dominik Moritz, Arvind Satyanarayan, and Jeffrey Heer (2018). Vega-Lite - A Grammar of Interactive Graphics. URL: https ://vega . github . io/vegalite/ (visited on Feb. 20, 2019) (cit. on p. 110). 


\section{Supplemental material}

All figures in this dissertation produced by code and data are open and reproducible. In addition, the supplemental material contains anonymized data from the interviews and the focus group. All URLs accessed at 01.04.2019. This dissertation includes the following supplemental materials.

Reproducible research and GIScience: an evaluation using AGILE conference papers

- List of references for the paper corpus: https://doi.org/10.7717/peerj. 5072/supp-1

- Survey questions: https://doi .org/10.7717/peerj.5072/supp-2

- Code and data for the analysis and the figures: https://doi.org/10.5281/ zenodo. 1227260

Computational reproducibility in geoscientific papers: Insights from a series of studies with geoscientists and a reproduction study

The Open Science Framework project https://doi.org/10.17605/OSF.IO/B2N78 includes:

- Questions, statements, codes, categories (to understand the grounded theory approach applied), and consent form for the interviews and the focus group

- List of references for the paper corpus

- Survey questions, data, and source code for the analysis and the figures

This paper is also available as an executable research compendium: https://o2r . uni-muenster.de/\#!/erc/81T5s. Note: Future accessibility might be affected by changes to the online platform and the underlying reproducibility service. The execution of the analysis requires a user registration using ORCID. 


\section{In-depth examination of spatiotemporal figures in open reproducible research}

Frontend implementation: https://github.com/o2r-project/o2r-platform

The Open Science Framework project https://doi.org/10.17605/OSF. IO/BRJTG includes:

- Questions, statements, codes, categories (to understand the grounded theory approach applied), and consent form for the interviews and the focus group

- Survey questions, data, and source code for the analysis and the figures

This paper is also available as an executable research compendium: https://o2r . uni-muenster.de/\#!/erc/82nd3. Note: see above.

\section{Creating interactive scientific publications using bindings}

Bindings implementation: https://github.com/o2r-project/o2r-bindings

The Open Science Framework project https://doi.org/10.17605/0SF.IO/EX9CP includes:

- Data and source code for the analysis and the figures

- A video showing how to create and use bindings

This paper is also available as an executable research compendium: https://o2r. uni-muenster.de/\#!/erc/GFqaR. Note: see above. 


\section{Curriculum \\ VITAE}

PERSONAL
INFORMATION
Name
Address
Phone
Email
Birthday
GitHub

\section{Profession}

$\triangleright$ Period

- Employer

- Position

$\triangleright$ Period

- Employer

- Position

$\triangleright$ Period

- Employer

- Position

$\triangleright$ Period

- Employer

- Position

\section{EDUCATION}

$\triangleright$ Period

- Qualification

- Institution

$\triangleright$ Period

- Qualification

- Institution

$\triangleright$ Period

- Qualification

- Institution

$\triangleright$ Period

- Qualification

- Employer
KONKOL, Markus

Rjasanstr., 9, 48147, Münster

(49) 15737 / 626471

m.konkol@uni-muenster.de

02.09.1987

https://github.com/MarkusKonk

01.03.2016-ongoing

Institute for Geoinformatics, Heisenbergstr. 2, 48149 Münster Research associate

01.10.2014-28.02.2015

Esri Germany GmbH, Ringstr. 7, 85402 Kranzberg

Trainee

$2012-2015$

Institute for Geoinformatics, Heisenbergstr. 2, 48149 Münster Student assistant

16.06.2010 - 31.08.2010

Atrios, Tiefe Str. 29, 48143 Rheine

Surveyor

01.03.2016-ongoing

Dr. rer. nat.

Institute for Geoinformatics, WWU Münster

01.10.2013-30.09.2015

Master of Science in Geoinformatics

Institute for Geoinformatics, WWU Münster

01.10.2010-30.09.2013

Bachelor of Science in Geoinformatics

Institute for Geoinformatics, WWU Münster

01.08.2007-31.07.2010

Surveyor

Surveying office Dr. Drees und Schlüter, Hohenzollernring 47, 48145 Münster 

